\title{
A Cavity Ring-Down Spectroscopy Mercury Continuous Emission Monitor
}

\section{Final Technical Report}

For the period

Starting October 1, 2001 Ending December 15, 2004

Work Performed Under Contract:

DE-FC26-01FT41221

Submitted By:

Sensor Research and Development Corporation

17 Godfrey Drive

Orono, Maine 04473

Christopher C. Carter, Ph.D.

Phone: $207 / 866-0100 \times 239$

Fax: 207/866-2055

Submitted To:

U. S. Department of Energy/National Energy Technology Laboratory Morgantown Energy Technology Center

Morgantown, West Virginia

COR: Susan Maley 


\section{Disclaimer:}

This report was prepared as an account of work sponsored by an agency of the United States Government. Neither the United States Government nor any agency thereof, nor any of their employees, makes any warranty, express or implied, or assumes any legal liability or responsibility for the accuracy, completeness, or usefulness of any information, apparatus, product, or process disclosed, or represents that its use would not infringe privately owned rights. Reference herein to any specific commercial product, process, or service by trade name, trademark, manufacturer, or otherwise does not necessarily constitute or imply its endorsement, recommendation, or favoring by the United States Government or any agency thereof. The view and opinions of authors expressed herein do not necessarily state or reflect those of the United States Government or any agency thereof. 


\begin{abstract}
:
The Sensor Research \& Development Corporation (SRD) has undertaken the development of a Continuous Emissions Monitor (CEM) for mercury based on the technique of Cavity Ring-Down Spectroscopy (CRD). The project involved building an instrument for the detection of trace levels of mercury in the flue gas emissions from coal-fired power plants.

The project has occurred over two phases. The first phase concentrated on the development of the ringdown cavity and the actual detection of mercury. The second phase dealt with the construction and integration of the sampling system, used to carry the sample from the flue stack to the CRD cavity, into the overall CRD instrument.
\end{abstract}

The project incorporated a Pulsed Alexandrite Laser (PAL) system from Light Age Incorporated as the source to produce the desired narrow band $254 \mathrm{~nm}$ ultra-violet (UV) radiation. This laser system was seeded with a diode laser to bring the linewidth of the output beam from about $150 \mathrm{GHz}$ to less than 60 $\mathrm{MHz}$ for the fundamental beam. Through a variety of non-linear optics the $761 \mathrm{~nm}$ fundamental beam is converted into the $254 \mathrm{~nm}$ beam needed for mercury detection.

Detection of the mercury transition was verified by the identification of the characteristic natural isotopic structure observed at lower cavity pressures. The five characteristic peaks, due to both natural isotopic abundance and hyperfine splitting, provided a unique identifier for mercury. SRD scientists were able to detect mercury in air down below 10 parts-per-trillion by volume (pptr). This value is dependent on the pressure and temperature within the CRD cavity at the time of detection.

Sulfur dioxide $\left(\mathrm{SO}_{2}\right)$ absorbs UV radiation in the same spectral region as mercury, which is a significant problem for most mercury detection equipment. However, SRD has not only been able to determine accurate mercury concentrations in the presence of $\mathrm{SO}_{2}$, but the CRD instrument can in fact determine the $\mathrm{SO}_{2}$ concentration as well. Detection of mercury down to the low hundreds of pptr has been accomplished in the presence of $\mathrm{SO}_{2}$ at concentration levels much higher than that found in typical flue gas emissions. SRD scientists extended the interferent testing to each individual component found in flue gas. It was found that only $\mathrm{SO}_{2}$ had a significant effect on the ring-down decay curve. Upon completion of testing the components of flue gas individually a simulated flue gas stream was used to test to the CRD instrument. The result showed accurate detection of mercury down to levels below 100 pptr in a simulated flue gas stream with the concentrations of the various components above that found in a typical untreated flue gas.

A sampling system was designed and integrated into the CRD instrument to carry the sample from the flue gas stack to the CRD cavity. The sampling system was constructed so that it could be placed very close to the sampling port. SRD scientists were able to couple the UV laser light into an optical fiber, which is then sent to the sampling system. This allows the laser system to be isolated from the sampling system. Initial long-term testing revealed a couple of problems related to the stability of the output frequency of the laser system. These problems have been successfully dealt with by incorporating specific software solutions into the overall data acquisition program.

The project culminated in a field test conducted at the DOE/NETL pilot plant facility in Pittsburgh, Pennsylvania. The object of the test was the evaluation of a cavity ringdown spectrometer constructed for the detection of TOTAL vapor phase mercury as a continuous emission monitor (CEM). Although there is the potential for the instrument to determine the amount of speciation between neutral elemental mercury $\left(\mathrm{Hg}^{(\mathrm{o})}\right.$ and oxidized mercury $\left(\mathrm{Hg}^{(+2))}\right.$, the initial test plan was to concentrate on the measurement 
of the total mercury. Another added benefit is that the measurements will report the sulfur dioxide (SO 2) concentration throughout the test.

This report concludes the technical work associated with Phases I \& II and the field test for the Cavity Ring-Down mercury detection project. 


\section{Table of Contents}

DISCLAIMER:

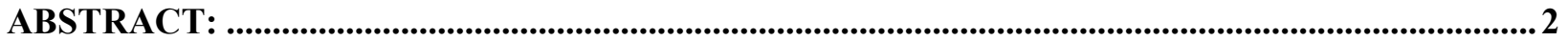

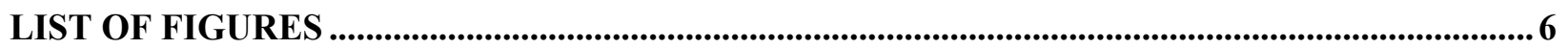

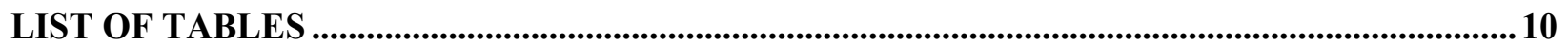

INTRODUCTION

GOVERNMENT AND INDUSTRY's PRESENT POSITION ON MERCURY CONTROL .........................................14

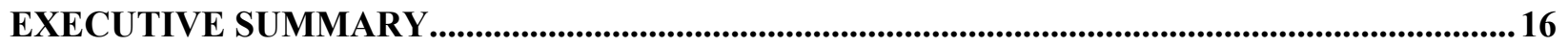

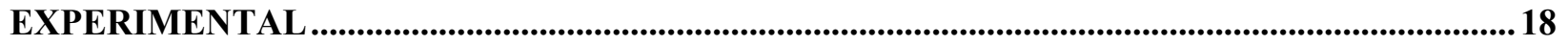

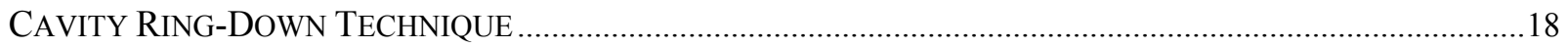

RESULTS AND DISCUSSION

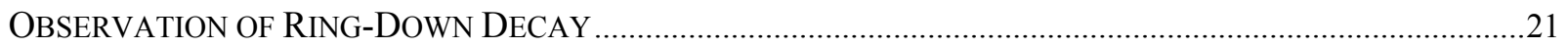

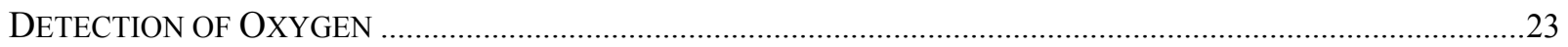

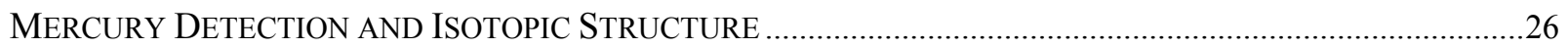

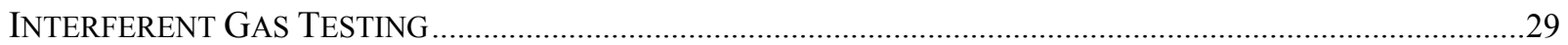

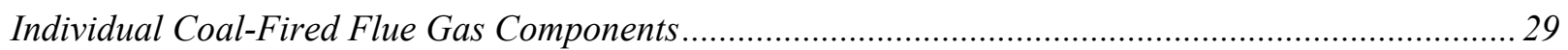

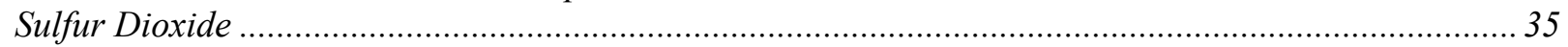

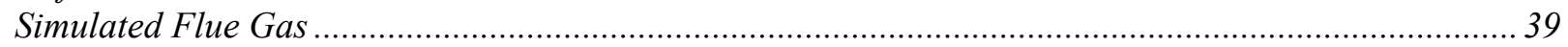

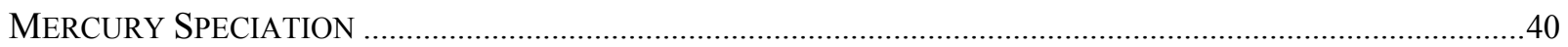

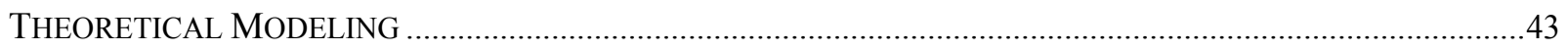

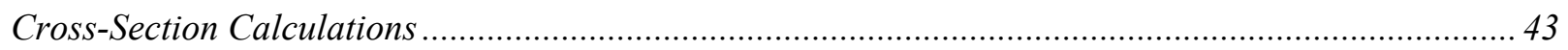

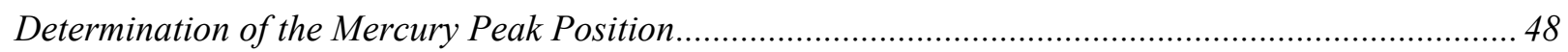

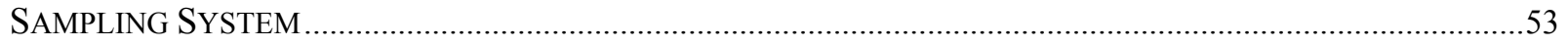

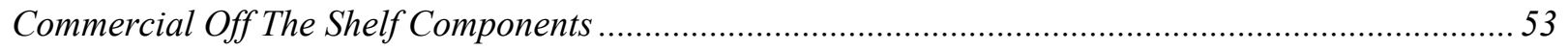

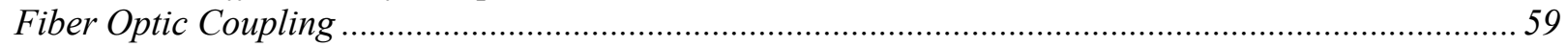

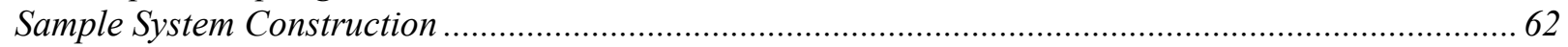

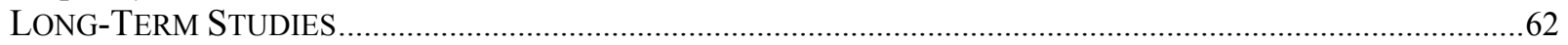

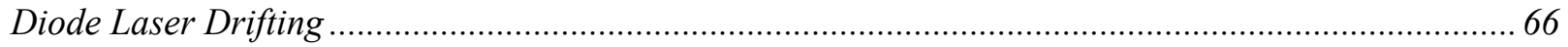

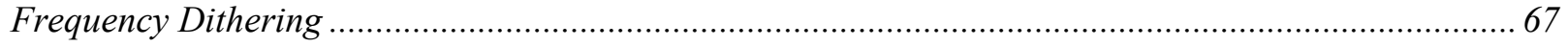

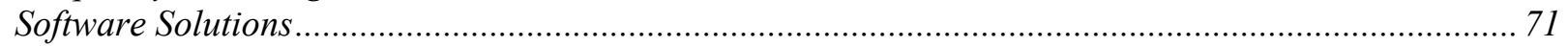

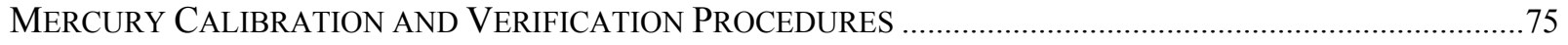

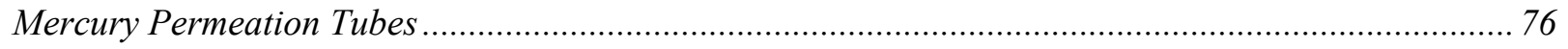

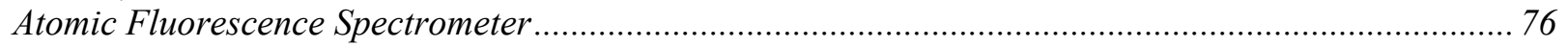

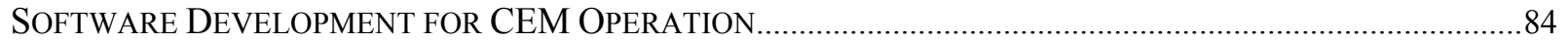

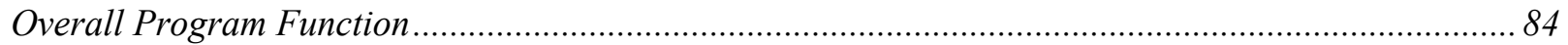

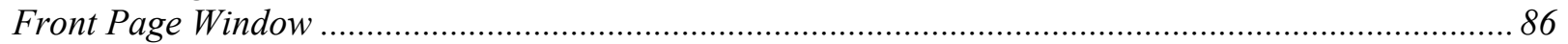

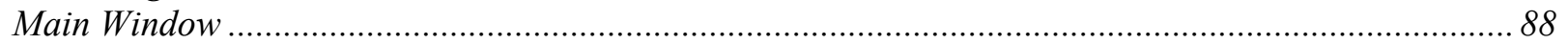

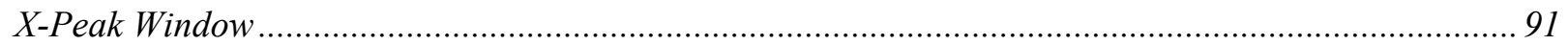

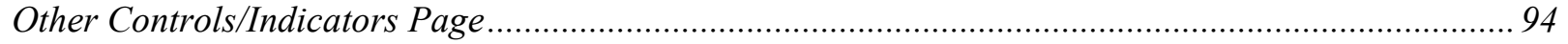




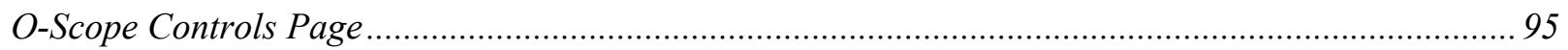

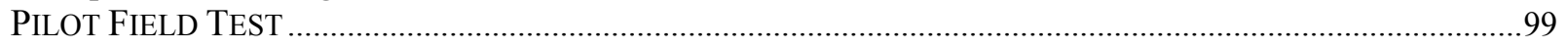

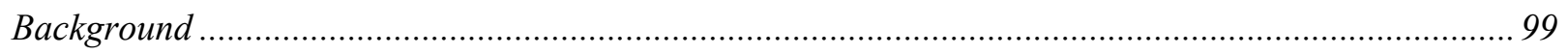

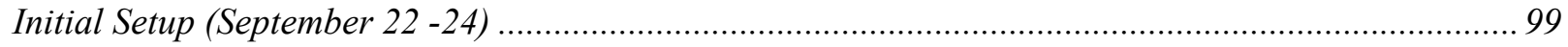

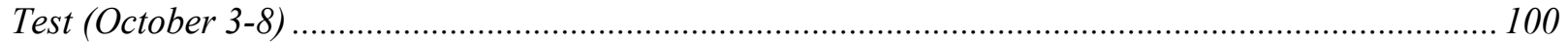

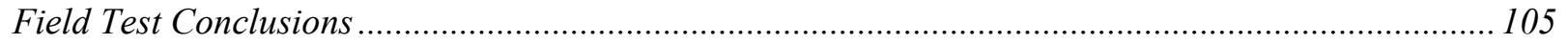

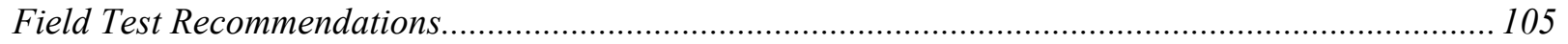

CONCLUSIONS, FUTURE WORK, AND COMMERCIAL POTENTIAL ..................................106

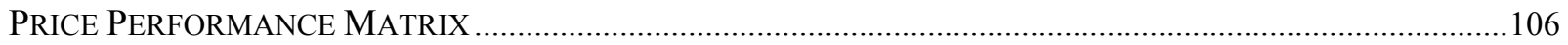

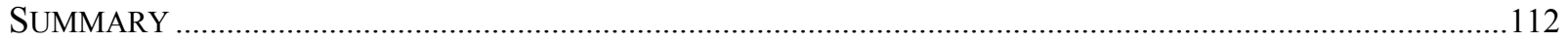

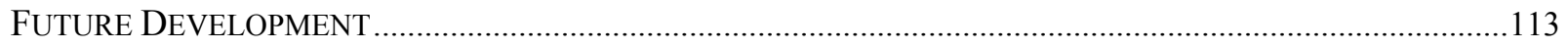

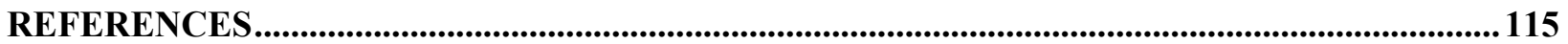




\title{
Sensor Research \& Development Corporation DE-FC26-01FT41221
}

\section{List of Figures}

Figure 1: Diagram of sampling cavity (not done to scale) placed between the two highly reflecting mirrors............19 Figure 2: Observed exponential ring-down decay with a fit curve plotted in red on top of the experimental plot. The derived ring-down time of just over $1 \mu \mathrm{sec}$ is consistent with the reflectivity reported for the mirror set by the

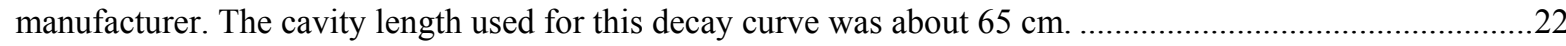

Figure 3: A portion of the $(7,0) \mathrm{A}-\mathrm{X}$ band of oxygen in the spectral region close to the $253.7 \mathrm{~nm}$ line of mercury. The scan range is about half of the total continuous range available with the diode seed laser or approximately $120 \mathrm{GHz}$ in the UV.

Figure 4: Scan of the seed diode laser voltage versus total losses with 400 torr of pure oxygen in the CRD cavity. The continuous scan covered the entire $\sim 250 \mathrm{GHz}$ spectral region possible to scan with the diode seed laser in a single scan.

Figure 5: Scans over the $(7,0)$ A-X oxygen band. The bottom plot (red) is a forward voltage scan $(-7$ to +7 volts $)$, while the top plot (blue) is a reverse scan of the voltage. The baseline level of the plots has been shifted for clarity. The applied voltage is directly proportional to the frequency output of the laser.

Figure 6: Scan of the oxygen transition originating from the ground $\mathrm{X}^{3} \Sigma_{\mathrm{g}}{ }^{-}$electronic state and terminating on one of 3 excited electronic states labeled on the plot. The absorption cross-section, $\sigma$, for the various transitions is given in $\mathrm{cm}^{2}$.

Figure 7: Scan of the $253.7 \mathrm{~nm}$ transition of atomic mercury. The pressure in the CRD cavity was 760 torr and the mercury concentration was about $1.5 \mathrm{ppb}$. The full-width half-maximum of the mercury line is approximately $22.5 \mathrm{GHz}$ due to the isotopic structure of mercury (see text) combined with pressure and Doppler broadening.

Figure 8: Schematic plot of the isotopic shifts and hyperfine splitting of the various isotopes of mercury. Also indicated in the plot is the expected linewidth for a single isotopomer line at 760 and 100 torr cavity pressure.

Figure 9: Scan of $\sim 500$ pptr of mercury at a cavity pressure of 100 torr showing the resolved isotopic structure of atomic mercury. The bars are the predicted natural abundances for each resolved isotopic peak. Due to the hyperfine splitting some of the peaks contain more than one single line resulting in 5 lines from 7 different isotopomers.

25


Figure 19: Scan over the atomic mercury isotopic structure with the $38 \mathrm{~cm}$ cavity held at a pressure of 50 torr and a temperature of $200^{\circ} \mathrm{C}$. The flow of gas contained $\sim 1.5 \mathrm{ppb}$ mercury and $\sim 1700 \mathrm{ppm} \mathrm{SO}_{2}$. The blue plot is the experimental data and the red plot is the theoretically predicted mercury spectrum..........................................39

Figure 20: Plot of mercury contained in a "simulated" flue gas. The sample gas stream contained $1.8 \mathrm{ppb}$ mercury, $1000 \mathrm{ppm} \mathrm{SO}, 70 \mathrm{ppm} \mathrm{NO}, 70 \mathrm{ppm} \mathrm{NO} \mathrm{N}_{2}, 285 \mathrm{CO}, 285 \mathrm{CO}_{2}$, and elevated levels of $\mathrm{H}_{2} \mathrm{O}$ from a bubbler. The cavity pressure was maintained at 100 torr and the temperature was held at $200^{\circ} \mathrm{C}$. The blue line is the experimentally determined losses from the ring-down signal and the red line is the calculated value using the experimental parameters including $1.8 \mathrm{ppb}$ mercury and $1000 \mathrm{ppm} \mathrm{SO}_{2}$.

ure 21: Plot of the total amount of mercury determined from both $\mathrm{HgCl}_{2}$ and elemental mercury. The $38 \mathrm{~cm}$ cell was held at a pressure of 100 torr and temperature of $200^{\circ} \mathrm{C}$. The concentration of the two mercury species was: $1 \mathrm{ppb} \mathrm{HgCl}_{2}$, and $1.5 \mathrm{ppb} \mathrm{Hg}$. The total concentration used in the calculated plot (red) was $2.5 \mathrm{ppb} \mathrm{Hg}$; the obtained ring-down data is shown in blue.

Figure 22: Scan of the oxygen transition originating from the ground $\mathrm{X}^{3} \Sigma_{\mathrm{g}}^{-}$electronic state and terminating on one of 3 excited electronic states labeled on the plot. The absorption cross-section, $\sigma$, for the various transitions is given in $\mathrm{cm}^{2}$. The experimental plot is shown in red while a theoretical plot is shown in blue. The $65 \mathrm{~cm}$ cell contained 400 torr of oxygen and was held at room temperature...

Figure 23: Scan of the $253.7 \mathrm{~nm}$ transition of atomic mercury. The pressure in the $38 \mathrm{~cm}$ CRD cavity was 760 torr, the temperature was held at $200^{\circ} \mathrm{C}$, and the mercury concentration was about $1.2 \mathrm{ppb}$. The full-width halfmaximum of the mercury line is approximately $22.5 \mathrm{GHz}$ due to the isotopic structure of mercury (see text) combined with pressure broadening.

Figure 24: Scan of $\sim 75$ pptr of mercury with the $38 \mathrm{~cm}$ cavity held at a pressure of 100 torr and temperature of $200^{\circ} \mathrm{C}$, showing the resolved isotopic structure of atomic mercury. The plot in blue is the experimental data while the plot in red is the theoretical prediction. Due to the hyperfine splitting some of the peaks contain more than one single line resulting in 5 lines from 6 different isotopomers......

Figure 25: A plot of the mercury concentration determined by the cavity ring-down instrument compared with the mercury concentration determined by the atomic fluorescence spectrometer (AFS).

Figure 26: Plot of a computer generated mercury spectrum (red dots) with a simulated noise level of $10 \%$ of the peak height. The solid blue line is a plot of the derivative of the spectrum. The vertical black dotted lines show the position where the derivative crosses the zero point. The parameters used for the simulation were a temperature of $200^{\circ} \mathrm{C}$, mercury concentration of $1.5 \mathrm{ppb}$, and a pressure of 100 torr.

Figure 27: Plot of a computer generated mercury spectrum (red dots) with a simulated noise level of $10 \%$ of the peak height. The solid blue line is a plot of the derivative of the spectrum. The vertical black dotted lines show the position where the derivative crosses the zero point. The parameters used for the simulation were a temperature of $200^{\circ} \mathrm{C}$, mercury concentration of $1.5 \mathrm{ppb}$, and a pressure of 400 torr.

Figure 28: Plot of a computer generated mercury spectrum (red dots) with a simulated noise level of $10 \%$ of the peak height. The solid blue line is a plot of the derivative of the spectrum. The vertical black dotted lines show the position where the derivative crosses the zero point. The parameters used for the simulation were a temperature of $200^{\circ} \mathrm{C}$, mercury concentration of $1.5 \mathrm{ppb}$, and a pressure of 760 torr...... . .51

Figure 29: Plot of actual data taken with the CRD apparatus shown by the red plot. The solid blue line is a plot of the derivative of the spectrum and the black dotted vertical lines are drawn through the point where the derivative spectrum crosses zero. The data was taken with a cavity pressure of 100 torr, a temperature of $200^{\circ} \mathrm{C}$, and a mercury concentration of $2.2 \mathrm{ppb}$.

Figure 30: Plot of actual data taken with the CRD apparatus shown in red dots. The solid blue line is a plot of the derivative of the spectrum and the black dotted vertical lines are drawn through the point where the derivative spectrum crosses zero. The temperature of the cavity was held at $200^{\circ} \mathrm{C}$, a pressure of 450 torr, and a mercury concentration of $1.7 \mathrm{ppb}$.

Figure 31: Plot of actual data taken with the CRD apparatus shown in red dots. The solid blue line is a plot of the derivative of the spectrum and the black dotted vertical lines are drawn through the point where the derivative spectrum crosses zero. The mercury concentration used was $1.7 \mathrm{ppb}$, the temperature of the cavity was held at $200^{\circ} \mathrm{C}$, and the pressure was maintained at 600 torr......

Figure 32: Diagram of the sampling system that will be used to extract a sample gas stream from the flue gas stack, carry it through the pyrolysis oven where all the mercury species are converted to elemental mercury, move it through the cavity where the mercury concentration is determine, and return the gas stream to the stack. The major components are listed on the figure... 
Figure 33: Plot of the relative frequency of the diode seed laser versus the losses in the cavity. The data was obtained with the cell maintained at room temperature, 870 torr pressure, and about $1.85 \mathrm{ppb}$ of mercury. The blue line is the experimentally obtained data and the red line is a theoretical plot fit to the parameters used in the experiment.

Figure 34: Plot of the relative frequency of the diode seed laser versus the losses in the cavity. The data was obtained with the cell maintained at room temperature, 90 torr pressure, and about $1.85 \mathrm{ppb}$ of mercury. The blue line is the experimentally obtained data and the red line is a theoretical plot fit to the parameters used in the experiment.

Figure 35: Plot of the relative frequency of the diode seed laser versus the losses in the cavity. The data was obtained with the cell maintained at room temperature, 100 torr pressure, and a flow rate of $1000 \mathrm{ccm}$ with 6.2 $\mathrm{ppb}$ of mercury. The blue dots are the experimentally obtained data and the red line is a theoretical plot fit to the parameters used in the experiment.

Figure 36: Diagram of the CRD sampling system coupled to the fiber optic carrying the UV laser pulse. The instrument is drawn on a 3' $\mathrm{x}$ 4' breadboard table. All the equipment on the table is drawn to scale.

Figure 37: Plot of the cavity losses versus time. The data was taken with the $38 \mathrm{~cm}$ cavity that was held at atmospheric pressure (760 torr) and the temperature of the cavity was held at $200^{\circ} \mathrm{C}$. The concentration of mercury from the permeation oven was kept at $1.25 \mathrm{ppb}$ in air. A data point was acquired every 2 seconds. The standard deviation of the plot is about $750 \mathrm{ppm}$ total cavity losses, which corresponds to a mercury concentration of about 30 pptr.

Figure 38: Plot of the cavity losses versus time. The cavity was maintained at room temperature, the pressure in the cell was held at 760 torr, and the mercury was constantly flowing through the cell with a concentration of 1.85 ppb. Each data point represents the average of 200 laser shots over a 4 second time interval..........................64

Figure 39: Plot of the cavity losses versus time. The cavity was maintained at room temperature, the pressure in the cavity was 760 torr, and the concentration of mercury at $\mathrm{t}=0$ was $6.2 \mathrm{ppb}$. The vertical line on the plot indicates the time at which the mercury supply was shut off.

Figure 40: Results of a 24-hour test monitoring mercury concentration. The mercury concentration was held at approximately $2.5 \mathrm{ppb}$ in air while the cell was at room temperature and 730 torr.

Figure 41: Plot showing the mercury concentration over time. The cell was kept at room temperature and atmospheric pressure. The mercury concentration was approximately $1.8 \mathrm{ppb}$. The observed oscillations are due to a frequency drift of the diode seed laser.

Figure 42: A scan of the laser frequency over the region of the mercury transition. The cell was held at room temperature and atmospheric pressure. The concentration of mercury was approximately $1.25 \mathrm{ppb}$. The vertical lines in the center of the plot shows the region that the diode laser was scanned back and forth across (see text).

Figure 43: Frequency dithering of the diode seed laser across the highest mercury isotopic peak (blue data). The cell was maintained at $58^{\circ} \mathrm{C}$ and a pressure of 187 torr. The mercury concentration used was about $1.5 \mathrm{ppb}$. The derivative plot is shown in green and the maximum determined from the derivative plot is shown as the points in red.

Figure 44: Plot of the maximum points determined for each scan across the highest mercury peak. These points are from the same plot that was shown in Fig. 43 but are shown for the first hour of the 5.5-hour test. .................69

Figure 45: Plot of the maximum points determined from the derivative of the ring-down data shown in Fig. 43. The plot displays all the maximum points obtained for the entire 5.5-hour test......................................................70

Figure 46: Spectrum observed from dithering the frequency back and forth over a single mercury isotopic peak. ...71

Figure 47: One of the windows available with the LabVIEW software used for obtaining CRD data. The window depicts a number of the options available and functions of the software (see text).

Figure 48: A second window used in the LabVIEW software for the CRD instrument. This window is used for finding a particular isotopic mercury peak to be used to monitor the mercury concentration............................73

Figure 49: Plot of an almost three day test. The mercury was held at a concentration of about $1.6 \mathrm{ppb}$, the temperature of the cell was maintained at $\sim 60^{\circ} \mathrm{C}$, and the pressure in the cell was 200 torr. The ${ }^{202} \mathrm{Hg}$ isotopic line was used to monitor the concentration. ...............................................................................................

Figure 50: A) Expanded plot of the 30 to 40 hour section of the plot shown in Fig. 49. B) Plot of the voltage value, supplied to the diode seed laser, where the program determines the center of the mercury transition to be........75

Figure 51: Schematic drawing of the AFS system with the various components listed on the figure........................79 


\section{Sensor Research \& Development Corporation DE-FC26-01FT41221}

Figure 52: Plot of the mercury concentration determined by the AFS versus that determined by the CRD instrument. The blue points are those determined by the CRD instrument. In the plot it is assumed that the AFS results are correct so is the blue points lie along the red line then the two agree.

Figure 53: Simplified flow diagram of the new program used to operate the CRD instrument as a continuous emissions monitor. The program is setup to obtain and report mercury concentration, on a continuous basis, as well as report the concentration of sulfur dioxide.

Figure 54: The Front display panel of the program used to operate the CRD instrument as a CEM. The tabs shown at the top left corner are the different displays viewable while the program is running and where a variety of parameters can be adjusted. Along the right side of the window is a display of various parameters that remain regardless of which tab is being viewed.

Figure 55: An example of the display observed on the Main page of the program. There are a number of plots displaying a variety of data used to determine the mercury concentration (see text).

Figure 56: The display shown for the X-Peak tab. There are a number of adjustable parameters viewable in the two boxes in the upper left corner of the display (see text). In this example the second mercury peak has been chosen as the monitoring peak for reporting the mercury concentrations.

Figure 57: An example of the Other Controls/Indicators page. This page, while not presently used, has been used in the past for data input into a second channel on the A/D converter.

Figure 58: The display observed for the O-scope page. This page contains all of the settings for the fast A/D card used to obtain the ring-down data. Also there are parameters that can be adjusted for the step size and number of steps taken for both the function that first finds the desired mercury peak, and for the function that scans back and forth across the desired mercury peak to obtain the mercury concentrations.......................................98

Figure 59: Fluctuations in mercury concentration due to laser misalignment......................................................102

Figure 60: Cavity ringdown measurements of the exhaust gas. .......................................................................102

Figure 61: Cavity ringdown measurements under optimum laser operation. ..........................................................103

Figure 62: Mercury spectra profile over $253.7 \mathrm{~nm}$ region at 400 Torr. Ran dynacalibrator output into the furnace w/flue gas.

Figure 63: Plot of the concentration of $\mathrm{Hg}$ versus time. Combined dynacalibrator output with flue gas. Cavity pressure 400 Torr...

Figure 64: A plot of price versus performance using the data from Table 3. Also indicated on the plot are 4 quadrants. These quadrants mark 4 general areas, where quadrant 1 represents the low cost and poor performance area, quadrant 2 is high cost and poor performance, quadrant 3 is high cost and superior performance, and quadrant 4 represents the low cost and superior performance area. 


\section{$\underline{\text { List of Tables }}$}

Table 1: Isotopic abundances of all naturally occurring isotopes of mercury...........................................................28

Table 2: Typical untreated flue gas composition from a coal-fired power plant burning low sulfur eastern bituminous coal. ${ }^{8}$

Table 3: Score of each instrument for all the different criteria and the final overall score for both performance and cost. 


\section{Sensor Research \& Development Corporation DE-FC26-01FT41221}

\section{$\underline{\text { Introduction }}$}

The objective of this project is an innovative science-driven technology program to develop an instrument that exploits the full potential of Cavity Ring-Down spectroscopy (CRD) as a powerful new analytical technique for the detection of ultra-trace levels of mercury.

One of the potential commercial applications of this CRD mercury detector is monitoring the flue gas emissions from coal-fired power plants. The EPA announced in Dec. 2000 that mercury emissions from these coal-fired power plants would be regulated. The EPA has set Dec. 2004 as the date when the exact emission requirements will be mandated and compliance will be required by 2007, under one of the proposed rules to reduce mercury emissions (see section on Government and Industry's Present Position on Mercury Control). The mercury emissions presently from these plants are fairly low, in the low partsper-billion by volume (ppb) range, and difficult to detect. The advent of any EPA regulations upon emissions of mercury, at whatever level is set, will require compliance and verification of emission levels. The present assessment of the government and the electric power generation industry is that there is not a single detector available that satisfies all of the mercury detection needs of coal-fired power plants.

Present detectors available on the market today are generally based on mature technologies such as atomic fluorescence, absorption, or emission. While able to detect mercury, these detectors are not able to handle the complex/harsh matrix of gases present in flue gas emissions. The majority of detectors require extensive sample pretreatment before being able to detect mercury. This extensive pretreatment can greatly increase the sampling time interval as well as bring into question the possibility of removing some mercury during the process. In addition to the pretreatment many detectors require time to concentrate the mercury to levels they are able to detect resulting in detectors that are not true continuous emission monitors.

Sensor Research \& Development Corporation (SRD) scientists and engineers have performed extensive testing of a mercury detection system, based on CRD. A prototype sampling system has been built which will take a sample from the environment and pass it through the ring-down cavity where the mercury content of the sample gas stream will be analyzed. SRD is currently working with the Department of Energy's National Energy Technology Laboratory (DOE/NETL) to bring this prototype device to a pilot plant facility in Pittsburgh, PA for the purpose of testing the CRD instrument on actual emissions from a pilot-scale coal-fired power plant. The pilot plant contains a $500 \mathrm{lb} . / \mathrm{hr}$. coal combustor. The CRD instrument will sample emissions downstream of the combustor at a couple of different locations. Results of the CRD instrument will be compared with those obtained by the traditional Ontario Hydro Method or EPA Method 29.

This report will cover a number of topics associated with the work performed under contract No. DEFC26-01FT41221. These include: a summary of the government's and industry's position on present and pending legislation dealing with mercury emissions from coal-fired power plants; a description of the cavity ring-down technique; a summary of the scientific work done on the development of the CRD instrument by SRD scientists and engineers; and a conclusion section summarizing the work done on Phases I \& II, detailing future work associated with the CRD project as well as the commercial potential, including a price performance matrix comparing a variety of presently available mercury detectors with the CRD instrument being developed by SRD, and path of development of the CRD trace mercury detection instrument by SRD. 
The core effort of the project focuses on the optimization and evaluation of a laboratory CRD spectrometer for gaseous mercury detection. Detection limits, calibration and appropriate analytical procedures have been established. The impact of individual interferent gases expected in flue gas emissions from advanced power plants has been determined. Upon testing of the individual gases a simulated flue gas steam consisting of a mixture of all expected flue gas components has been tested to determine combined interferences as well as mercury detection limits in the presence of various interferents. While the primary concern of this project is the determination of the total mercury content of flue gas emissions, approaches to determine mercury speciation has also been investigated. A sampling interface has been designed, built, and integrated into the CRD spectrometer to provide a continuous sample stream from the flue gas stack to the cavity of the CRD instrument.

A summary of the major tasks and subtasks involved in the project are described below:

Task 1: Design and Construct CRD System for Optimum Mercury Detection (1-Oct-2001 to 31Mar-2002)

1.1 Determine hardware requirements for prototype

1.2 Design and optimize laser for operation at $254 \mathrm{~nm}$

1.2.1 Establish laser linewidth

1.2.2 Establish mode stability

1.3 Evaluate optical cavity designs (maximize stability and sensitivity)

1.4 Finalize CRD design

1.5 Purchase CRD components

1.6 Construct Cavity

1.7 Establish baseline properties of spectrometer

1.7.1 Establish mirror reflectivity

1.7.2 Establish empty cavity performance

Task 2: Develop Supporting Software (1-Oct-2001 to 31-Jan-2002)

2.1 Develop LabVIEW software package to control the $50 \mathrm{~Hz}$ laser and sampling system

2.2 Modify existing LabVIEW software for analyzing data

Task 3: Hg-CRD System Optimization (1-Apr-2002 to 15-Mar-2004)

3.1 Characterize and optimize operating system

3.1.1 Establish technique sensitivity and detection limits

3.1.2 Evaluate effect of typical interferents and mitigation techniques

3.2 Evaluate approaches to $\mathrm{Hg}$ speciation (oxidized molecular species from neutral elemental mercury)

Task 4: Sampling Interface Design (1-Apr-2002 to 15-Jun-2003)

4.1 Evaluate commercially available sampling systems

4.2 Review results obtained under Task 3 to determine properties required for a thermal treatment CEM

4.3 Design sample system for on-line CRD CEM

Phase I (15-June-2003)

Complete Phase I

Submit continuing application

Obtain DOE/NETL approval for Phase I progress and Phase II activities 
Phase II (15-Jun-2003)

Begin Phase II

Task 5: Integrate Sampling System with CRD Instrument and Determine Combined Performance (1-Jul-2003 to 15-Mar-2004)

5.1 Construct sample system for on-line CRD CEM

5.1.1 Integrate sample system with CRD instrument

5.2 Evaluate performance of integrated system

5.2.1 Determine and minimize the impact on the CRD instrument's sensitivity caused by the sample system

5.2.2 Determine and minimize the impact on the CRD instrument detection limits caused by the sample system

5.3 Establish calibration procedures and operational parameters

Task 6: Formalize Hg-CRD Analytical Procedures (1-Oct-2003 to 15-Mar-2004)

6.1 Document calibration procedures

6.2 Document operating parameters

Task 7: Reporting (1-Oct-2001 to 15-May-2004)

7.1 Prepare publications and presentations, as appropriate, to disseminate results

7.2 Prepare and submit required status reports to communicate progress to NETL

7.3 Formalize and document operating and calibration procedures

7.4 Prepare and submit final report 


\section{Sensor Research \& Development Corporation DE-FC26-01FT41221}

\section{Government and Industry's Present Position on Mercury Control}

On 15-Dec-2003 the Environmental Protection Agency (EPA) proposed a rule to permanently cap and reduce mercury emissions from coal-fired power plants. The EPA will take comments on the two proposed emission reduction methods for 60 days from its publication in the Federal Register at the end of January 2004. EPA will also hold two public hearings on the proposed rules. The regulations for mercury emission control will be finalized on or before 15-Dec-2004.

The EPA has proposed two alternative solutions for controlling mercury emissions from power generating facilities. These two alternatives include:

1) Proposed rule requiring utilities to install controls known as "maximum achievable control technologies" (MACT) under section 112 of the Clean Air Act (CAA); and

2) Proposed rule establishing "standards of performance" limiting mercury emissions from new and existing utilities. This proposal under section 111 of the CAA, would create a market based "cap-and-trade" program that would take place in two phases.

The CAA of 1990 required the EPA to complete two studies related to mercury and report the results to congress. In 1994, through a couple of legal settlements, the deadlines to complete these studies were revised. In addition, the EPA agreed to determine if MACT regulations were appropriate and necessary, and if needed the EPA would propose a MACT standard. In December of 1997 the EPA issued a report entitled "Mercury Study." This report analyzed mercury emissions from utilities and other industrial sources, evaluated the health and environmental impacts of those emissions and available control technologies. The EPA issued a second report, "Utility Report," to congress in February 1998. In this report the EPA analyzed emissions of toxic air pollutants, including mercury, from utilities. In 1998 the EPA agreed to issue its regulatory determination by December 2000; to propose regulations by December 15, 2003; and to finalize regulations by December 15, 2004. In December 2000 the EPA announced it would regulate emissions of mercury and other air toxics from coal- and oil-fired utilities under section 112 of the CAA, or rather, impose some level of MACT standard.

In a closely related action on December 17, 2003, the EPA proposed the "Interstate Air Quality Rule." This proposal is for the reduction of both sulfur dioxide $\left(\mathrm{SO}_{2}\right)$ and nitrogen oxides $\left(\mathrm{NO}_{\mathrm{x}}\right)$. This is also a two-phase program that would result in two levels of reduction for both species, first in 2010 and then again in 2015. To achieve the required reduction the proposal suggests that individual states regulate utilities under a cap-and-trade program. The EPA is proposing to address the reduction of mercury along with $\mathrm{SO}_{2}$ and $\mathrm{NO}_{\mathrm{x}}$ in an integrated fashion.

Under the MACT proposal regulations would be set for mercury control (among a number of other hazardous air pollutants [HAP]). Under MACT there would be plant-by-plant control requirements that are technology based. The focus would be on Mercury/HAP control with some level of co-benefits. Under the MACT provision, after the rule is finalized in December 2004 sources are given 3 years for compliance. However, in general a one-year extension is usually granted for such changes. If implemented, this proposal would reduce mercury emissions by $29 \%$ by 2007 .

The second proposed rule is a cap-and-trade program that would occur over two phases. The first phase would take place by 2010 where mercury reductions would be accomplished by "co-benefit" control. That is, reductions in mercury emissions as a result of enacting the Interstate Air Quality Rule for reduction of $\mathrm{SO}_{2}$ and $\mathrm{NO}_{\mathrm{x}}$. The second phase of the program would establish a mandatory cap on mercury emissions 
by the year 2018. Under this cap-and-trade program the EPA would allocate each individual state a specific amount of emission "allowances" for mercury. The states would allocate these allowances to the individual utilities, which could then trade them. A specific utility must have enough allowances to cover its emissions each year. If implemented, this proposal would reduce mercury emissions by $69 \%$ by 2018 .

The EPA is also proposing to revise the December 2000 finding that it is "appropriate and necessary" to regulate utility hazardous air emissions using the MACT standards provision of the CAA under section 112. This would prevent promulgation of the MACT standards due to take effect.

Environmental groups generally believe that imposing a MACT standard would provide for a greater reduction of Mercury/HAPs emission sooner. The Electric Generating Industry, and the present administration, prefers the extended compliance window coupled with the cap-and-trade program.

In either event there is a great deal of interest from the energy producers of this country, as well as Europe, for both accurate reliable monitoring equipment and mercury control technology. Presently there is general agreement from everyone that there is not a mercury continuous emissions monitor on the market that satisfies industry's or the EPA's requirements. Indeed, the proposed rules published in the Federal Register require monitoring of stack emissions by mercury continuous emissions monitors (CEMs). 


\section{Sensor Research \& Development Corporation DE-FC26-01FT41221}

\section{Executive Summary}

The Sensor Research \& Development Corporation (SRD) has completed the second phase of a project for the Development of a Continuous Emissions Monitor (CEM) for Mercury. The goal of the first two phases of the project was to develop an instrument to detect trace levels of mercury in the flue gas emissions of coal-fired power plants using the relatively new analytical technique of Cavity Ring-Down (CRD) Spectroscopy.

A number of recent events point to the timeliness of this project, such as: recent findings by the Environmental Protection Agency (EPA); pending legislation; setting of emission limits; and emerging requirements for regulatory compliance of mercury emissions. In addition, this project positions SRD to be at the forefront in technological development of mercury detection equipment at a time when the hazardous effects of mercury in the environment are becoming a more prevalent concern for the people of the United States and around the world.

The project was initiated with the setup of the Alexandrite laser system that was used to produce the 254 $\mathrm{nm}$ Ultra Violet (UV) radiation that is required for detection of elemental gas-phase mercury. A cavity was designed and built by SRD engineers after which the first ring-down events were recorded. This led to the detection of a molecular oxygen transition spectrally adjacent to the mercury transition. The oxygen transition provided a good diagnostic signal that was used to optimize the operation of the CRD instrument before mercury was introduced into the system.

The next phase of the project involved using the optimized CRD instrument to detect mercury. Mercury was introduced into the ring-down cavity and the characteristic mercury isotopic pattern was obtained. A theoretical model has been developed by SRD scientists that accurately models the mercury transition, with its various isotopic peaks, for any temperature and pressure. This model is used to determine precise mercury concentrations. Verification of the mercury concentrations delivered to the CRD instrument was done by a couple of methods. The first was to use certified mercury permeation tubes as the source of mercury sent to the CRD apparatus. These permeation tubes were contained in a temperature-controlled oven, usually around $50-100^{\circ} \mathrm{C}$, which resulted in a constant permeation rate of mercury. Verification of the permeation rates, as well as samples taken throughout the sample path to ensure no mercury losses within the CRD instruments tubing or cavity was done with an Atomic Fluorescence Spectrometer (AFS). The AFS instrument was calibrated with headspace measurements from mercury contained in a vial held in a constant temperature bath.

One of the problems encountered by most mercury detection instruments today is that of interferents contained in the same sample gas stream as the mercury. Flue gas emissions from coal-fired power plants contain a complex matrix of hazardous gases in varying concentrations. Presently available mercury detection equipment requires extensive pretreatment of this gas stream prior to mercury detection. SRD has been able to detect mercury, down into the low parts-per-trillion by volume range, in a simulated flue gas stream with no sample pretreatment. This includes tests using a variety of excessively high concentrations of sulfur dioxide, which is one of the most problematic of the flue gas components for mercury detection.

SRD also investigated the possibility of spectrally speciating between the elemental form of mercury, $\mathrm{Hg}^{(0)}$, and the oxidized form of mercury, $\mathrm{Hg}^{(+2)}$. While SRD scientists have not been able to spectrally speciate these two forms of mercury work continues along this avenue, as this is important information that would be of benefit to the power generating industry. The CRD instrument is presently able to infer the concentration of these two forms of mercury but it would be preferable to directly detect the oxidized form in the same way as the elemental form. 
SRD engineers have designed and built a sampling system that will be used to take a sample gas stream from a flue gas stack, flow it through a pyrolyzer where all forms of mercury will be converted to elemental, carry it to the ring-down cavity where the total gas-phase mercury concentration will be determined, and return the gas to the flue gas stack. This system has been built on a relatively small breadboard in order to be portable to move from sampling point to sampling point.

A number of long-term studies have been conducted with the CRD instrument, as it will be required to continuously monitor mercury concentrations over extended periods of time. A couple of problems were encountered with the diode laser that seeds the Alexandrite laser system. Both problems had to do with frequency stability of the diode laser. One problem resulted from the diode laser slowly drifting in frequency over time. This meant that the UV light used to monitor the mercury concentration was no longer sitting on top of the mercury peak and hence the CRD program reported erroneous concentration values. The second problem was caused by mode hops of the diode laser, which resulted in a large, sharp frequency jump, again resulting in incorrect mercury measurements. Both problems have been dealt with through additional subroutines to the overall CRD program that ensure the UV laser frequency remains on the mercury peak and accurate mercury concentrations are reported.

Due to the success attained in the development of the CRD instrument, SRD and the Department of Energy's National Energy Technology Laboratory (DOE/NETL) are continuing the development of the ultra trace level mercury instrument. This continued development is moving into the pilot-scale testing phase. SRD will perform two field tests at the DOE/NETL facility in Pittsburgh Pennsylvania. The tests will involve a continuous weeklong sampling time at two different locations along the flue gas stream. A successful completion of these tests might possibly lead to a test at a full-scale power plant.

After completion of these field tests SRD will be in a position to fully commercialize the CRD instrument. Part of this process could involve participation in the Environmental Protection Agency's Environmental Technology Verification program. This program provides an independent comparison of a variety of commercially available mercury detection instruments throughout a full-scale test at a power generating facility. In addition to the development of the CRD cavity, software, and sampling system, SRD is continuing to work with Light Age Incorporated to reduce the cost and size of the laser source used to produce the narrow band $254 \mathrm{~nm}$ UV light. 


\section{Sensor Research \& Development Corporation DE-FC26-01FT41221}

\section{Experimental}

A problem that exists with present day mercury detection equipment is the need for sample pre-treatment of the complex flue gas matrix to eliminate all interferences by species such as sulfur dioxide. This extensive sample pre-treatment results in instruments that function more as semi-continuous emission monitors rather than true Continuous Emission Monitors (CEMs). It also brings into question possible mercury losses and changes in the different types of mercury (neutral elemental and oxidized) during this sample pre-treatment before analysis.

The Sensor Research \& Development Corporation's solution to this problem is to use a relatively new absorption technique known as Cavity Ring-Down spectroscopy (CRD). As a laser-based technique it is inherently very selective to the species of interest. The laser system that is used for this project was a pulsed Alexandrite laser that runs at $50 \mathrm{~Hz}$, which means that the time between measurements can be as little as 20 milli-seconds, or essentially a real-time continuous measurement of the mercury concentration. A description of the CRD technique used for this project is given below.

\section{Cavity Ring-Down Technique}

Cavity Ring-Down spectroscopy is a sensitive absorption technique that was first developed by O'Keefe and Deacon in 1988. ${ }^{1}$ This technique injects a pulse of laser light into a stable optical cavity formed by two highly reflecting mirrors. The light reflects back and forth in the cavity resulting in extremely long effective pathlengths. Using mirrors with a reflectivity of $99.99 \%$, which is typical for the visible range of the spectrum, and a 1-meter long cavity it is possible to achieve an effective pathlength of 10 kilometers, during the first 1/e time of the decay of the ring-down signal. As the light reflects back and forth in the cavity a small amount of light is transmitted through the end mirror of the cavity to a photon detector, such as a photomultiplier tube. The light exiting the cavity decays exponentially with time at a rate determined by round trip loss mechanisms within the cavity. The measured time constant for the exponential decay of light, or the time taken to reach the 1/e time of the decay, is called the "ring-down time" of the cavity. The dominant loss mechanism for an empty cavity is the mirror transmission. However, if a sample species, which absorbs light at the particular wavelength injected into the cavity, is placed within the cavity, the ring-down time will decrease from that of the empty cavity at that particular wavelength. The ring-down time is given by

$$
\tau=\frac{l_{c}}{c\left[(1-R)+\alpha_{s} l_{s}+\alpha_{b} l_{c}\right]}
$$

where, $l_{c}$ is the cavity length, $c$ is the speed of light, $R$ is the reflectivity of the mirrors, $\alpha_{\mathrm{s}}$ is the absorption coefficient of the sample species of interest, $l_{s}$ is the pathlength through the sample, and $\alpha_{\mathrm{b}}$ is the absorption coefficient for various background losses. Background losses, which are all included in the last term of the denominator of eq. 1, can include scattering losses due to Mie or Rayleigh scattering or absorptions due to other components in the sample gas stream. Once the empty cavity losses and any other background losses have been determined, CRD spectroscopy provides an absolute measure of the concentration of the absorbing sample of interest within the cavity. This self-calibrating feature differentiates CRD from other highly sensitive laser-based methods such as laser induced fluorescence (LIF) or resonantly enhanced multiphoton ionization (REMPI). ${ }^{2}$ This self-calibrating feature means that no calibration curves are necessary to determine absolute concentrations of the sample species of interest.

The laser source that was used for the project is a pulsed Alexandrite ring laser (Light Age Inc., PAL101). This is a solid-state laser that runs at $50 \mathrm{~Hz}$, will produce pulse energies of $>0.5 \mathrm{~mJ} / \mathrm{pulse}$ in the 
Ultra-Violet (UV) region of the spectrum at $253.7 \mathrm{~nm}$, and has a fundamental laser linewidth of about 150 GHz. A diode seed laser is used with the system to bring the fundamental linewidth down to approximately $60 \mathrm{MHz}$, which results in a laser linewidth of about $180 \mathrm{MHz}$ at $253.7 \mathrm{~nm}$. This laser pulse was spatially filtered and mode matched to the particular ring-down cavity that was used. Initial results indicated a cavity length of about $65 \mathrm{~cm}$ is optimum for the CRD setup using specially coated planoconcave mirrors with a $6 \mathrm{~m}$ radius of curvature. However, after initial work was done with a $65 \mathrm{~cm}$ cavity length, a final cavity length of $38 \mathrm{~cm}$ was used. The reduced length still maintained low parts-per-trillion by volume (pptr), but offered a smaller footprint for the final prototype.

The design of the CRD absorption cell has an inlet for the sample containing mercury and exit for a continuous flow. The design was such that a low flow of inert gas can be sent over the face of the highly reflecting mirrors to insure no degradation of the mirror surface, due to deposited contaminants, and hence a decrease in the ring-down time resulting in a decrease of the sensitivity of the CRD instrument. A diagram of the absorption cavity is shown in Fig. 1. From the figure it can be seen that the flow of sample flue gas enters the cavity near the end and is exhausted from the center of the cavity to maintain the gas flow away from the mirror surfaces.

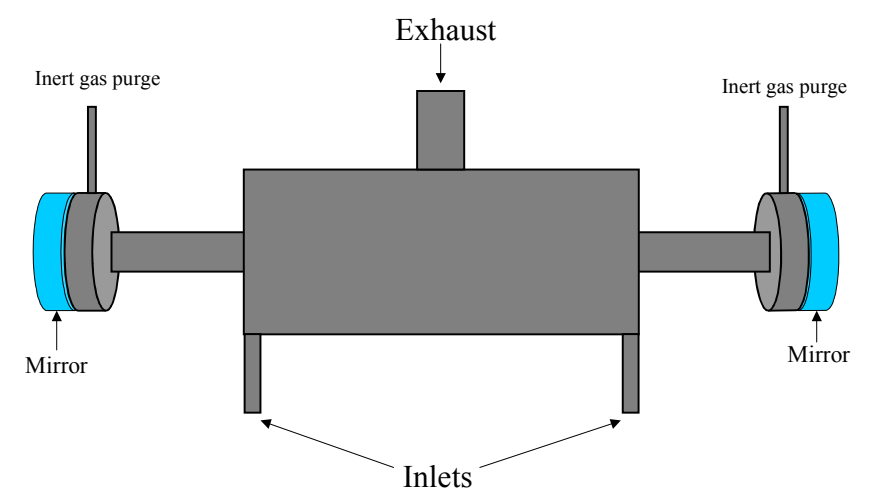

Figure 1: Diagram of sampling cavity (not done to scale) placed between the two highly reflecting mirrors.

The data acquisition system takes the output of a photomultiplier tube and sends it to a fast analog-todigital 12-bit, $100 \mathrm{MHz}$ card (Gage Applied Sciences Inc., CS12100). A LabVIEW (National Instruments Corp.) program was written for control of the laser system, data acquisition, and signal processing. The program calculates the decay time of the ring-down curve, compares it with that for the empty cavity, and determines the absolute concentration of mercury in the absorption cell at any time.

A mercury vapor generation system was used to generate known amounts of mercury for testing as well as a periodic calibration check of the CRD instrument. The vapor generation system consists of a temperature-controlled oven within a dynacalibrator (Valco Instruments Co. Inc., Model 500) with a mercury permeation tube of a known permeation rate. Additional verification of the mercury concentration delivered to the CRD instrument was done using an Atomic Fluorescence Spectrometer (AFS) (Brooks Rand LTD, Model III). The AFS is calibrated using a gold trap with injections of mercury vapor from the headspace of a liquid mercury container held at a known constant temperature. 
Mercury speciation studies were accomplished using an oven pyrolyzer system (Kendro Laboratory Products Inc., Lindberg/Blue M Model TF55030A). The relative efficiency of the pyrolyzer for dissociating different species of mercury was evaluated using known quantities of substances such as elemental mercury and mercuric chloride from permeation tubes. The pyrolyzer allows the detection of the total amount of mercury in a sample stream by dissociating any mercury compounds, or oxidized forms of mercury, to neutral elemental mercury. Further speciation studies looked at the possibility of spectrally differentiating compounds containing mercury from elemental mercury. A published study that investigated the spectral separation of mercury and mercury chloride concluded that there may have been a slight spectral shift but they were unable to resolve the small frequency shift with their laser system. ${ }^{3}$ The diode seeded Alexandrite system utilized for this project has a spectral resolution over 20 times narrower than the above-mentioned study. This increase in resolution may well allow us to spectrally separate elemental mercury from compounds of mercury. 


\section{$\underline{\text { Results and Discussion }}$}

The scientific results and discussion from the first two phases of the CRD project are given below. The ordering and description of events that have occurred throughout the project is essentially chronological, however some of the discoveries may be somewhat out of chronological order in the interest of continuity and clarity.

\section{Observation of Ring-Down Decay}

Initial tests to detect a ring-down signal were completed after the cavity to be used for the ring-down measurements of mercury was built. This also includes the pumps and delivery system for mercury and possible interferent gases.

The reproducibility of the laser system, in terms of power stability and alignment, with the CRD cavity was investigated. After an initial warm-up period the power level of the laser remained constant $( \pm 10 \%)$ over an entire day of continuous operation. The laser power and pointing stability remained constant, after the initial warm-up, for a period of a week without adjustments to the laser alignment. The ring-down cavity itself would also be stable for an entire day of continuous operation although the alignment of the cavity mirrors was generally adjusted on a daily basis.

The effect of Rayleigh scattering due to an ambient atmosphere between the cavity mirrors was investigated. The wavelength used for mercury detection is well into the Ultra-Violet (UV) region (253.7 $\mathrm{nm}$ ) of the spectrum, and as the Rayleigh scattering intensity is inversely proportional to the fourth power of the wavelength, this could cause a significant decrease in the overall ring-down time thereby reducing sensitivity. However, experimentally we observed that there was no significant decrease in the overall ring-down time due to Rayleigh scattering. This was checked experimentally by observing ring-down times in the ambient atmosphere and also with an evacuated cavity.

An example of a ring-down decay can be seen in Fig. 2 shown below. In the figure is an experimentally observed ring-down decay. Plotted on top of the decay, in red, is a fit of the exponential decay. The fit gives a ring-down time slightly longer than $1 \mu \mathrm{sec}$, with a cavity length of $65 \mathrm{~cm}$. Using standard ringdown expressions and inputting the ring-down time, the length of the cavity, and the speed of light, the empty cavity losses are calculated to be $99.78 \%$. This was done for an evacuated cavity so the major source of loss was due to the mirror reflectivity. The reported reflectivity for the mirrors, by the manufacturer, is $99.7 \%$, which is entirely consistent with our results. This indicated that there were no additional losses caused by the ring-down cavity constructed at SRD and that everything was operating as expected.

Data acquisition software was written that controls recording of ring-down events through the $100 \mathrm{MHz}$ 12-bit A/D card. The program has been written in LabVIEW. The same LabVIEW software interfaces a fitting routine with the output of the data acquisition card to calculate the losses of the cavity as a function of time or wavelength. Additionally, the program controls scanning of the diode laser, which seeds the Alexandrite ring laser. 


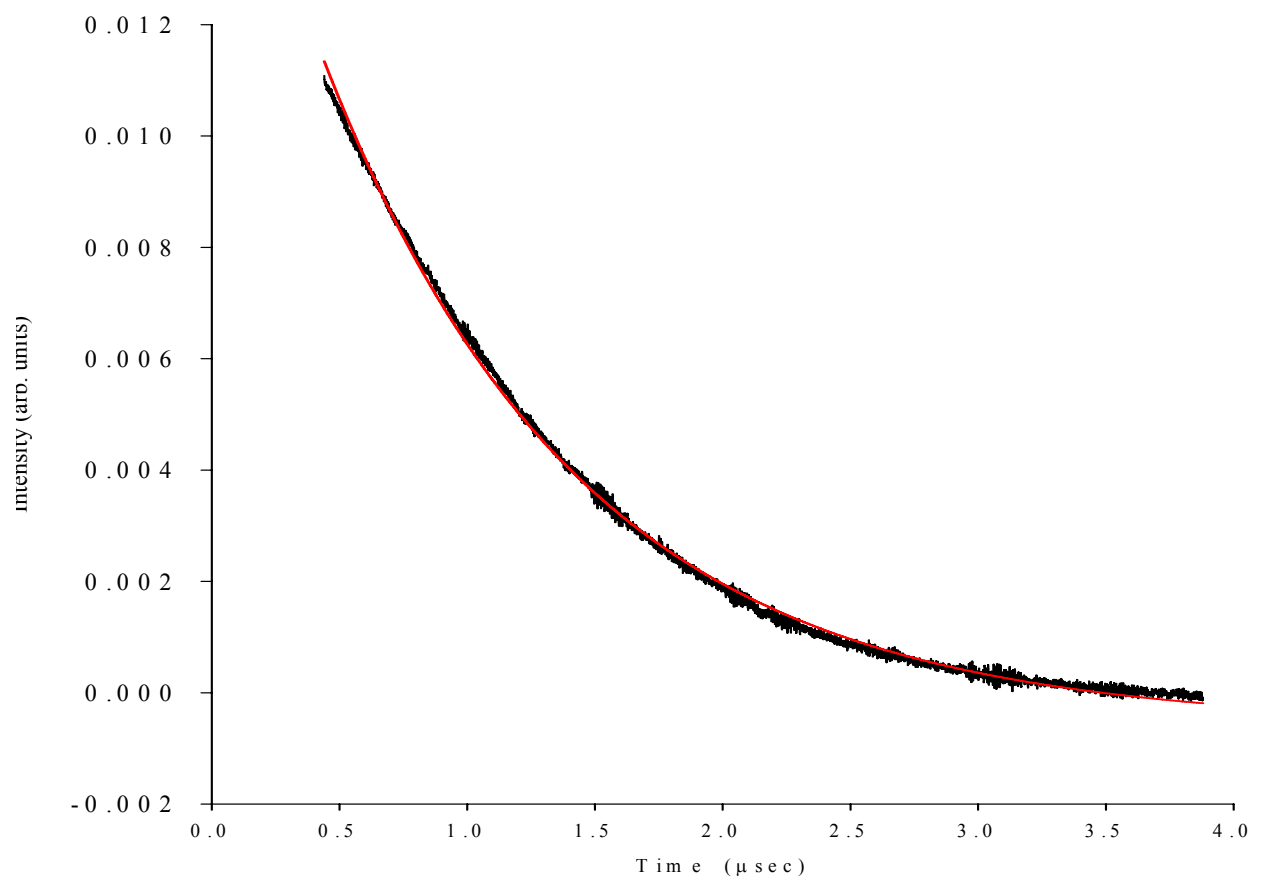

Figure 2: Observed exponential ring-down decay with a fit curve plotted in red on top of the experimental plot. The derived ring-down time of just over $1 \mu \mathrm{sec}$ is consistent with the reflectivity reported for the mirror set by the manufacturer. The cavity length used for this decay curve was about $65 \mathrm{~cm}$.

In an effort to further refine the cavity to be used for the ring-down measurements SRD conducted a number of studies using a variety of cavity lengths. The initial cavity length used was approximately 65 $\mathrm{cm}$ long. One of the questions that arise using cavity ring-down spectroscopy, with a relatively narrow linewidth laser system, is the ability to maintain continuous transmission through the cavity when the linewidth of the laser no longer overlaps one or more modes of the cavity. Theoretically, with a high finesse cavity a single mode laser can only have transmission when the laser linewidth overlaps one or more of the cavity modes, however, excitation of additional transverse cavity modes can lead to continuous transmission. This effect has been demonstrated by Gerard Meijer et al. who used a very short cavity $(10 \mathrm{~cm})$ with a very narrow linewidth laser $(5 \mathrm{MHz})$ and were able to demonstrate continuous transmission. ${ }^{4}$ SRD has evaluated a number of different cavity lengths. Cavities with lengths down to 12 $\mathrm{cm}$ were tested. SRD was able to obtain continuous transmission with all cavity lengths tested, over the frequency range needed to observe the mercury transition. Taking into consideration a number of factors such as, detection limits, effects of $\mathrm{SO}_{2}$ on the baseline level, laser light transmission, and overall size of the CRD instrument, the previously used $65 \mathrm{~cm}$ cavity was replaced with a cavity slightly less than 40 $\mathrm{cm}$. 


\section{Detection of Oxygen}

With all the data acquisition pieces in place the CRD cell was filled to atmospheric pressure (760 torr) with natural air. The spectral region near the $253.7 \mathrm{~nm}$ mercury line contains a band of molecular oxygen $\left(\mathrm{O}_{2}\right)$. This band of oxygen is a very weak absorption band as it is an electronic dipole forbidden transition. The transition is observable due to perturbations by a number of close lying electronic states. The transition is the $(7,0)$ vibrational band of the $A^{3} \Sigma_{\mathrm{u}}^{+}-X^{3} \Sigma_{\mathrm{g}}^{-}$electronic transition, commonly know as the Herzberg I transition. ${ }^{5}$

Shown in Fig. 3 is a plot of a couple of rotational transitions of the above-mentioned transition in molecular oxygen. This is a scan over only a part of the $\sim 250 \mathrm{GHz}$ region that can be covered by the diode laser in one continuous scan. The plot is shown as overall cavity losses in parts-per-million (ppm) versus voltage applied to the diode seed laser, which corresponds to a change in wavelength of the diode laser.

After this observation, to confirm that the observed ring-down signal was indeed that due to the weak oxygen transition that it was believed to be, the cell was pumped out and the region was scanned again to insure a flat baseline. A cylinder of high purity oxygen was then used to fill the CRD cell with 400 torr of pure oxygen. The region was scanned again with the results plotted in Fig. 4. In this plot the diode laser was scanned over the full continuous range. From the plot it can be seen that the observed rotational lines do belong to the weak $\mathrm{O}_{2}$ transition.

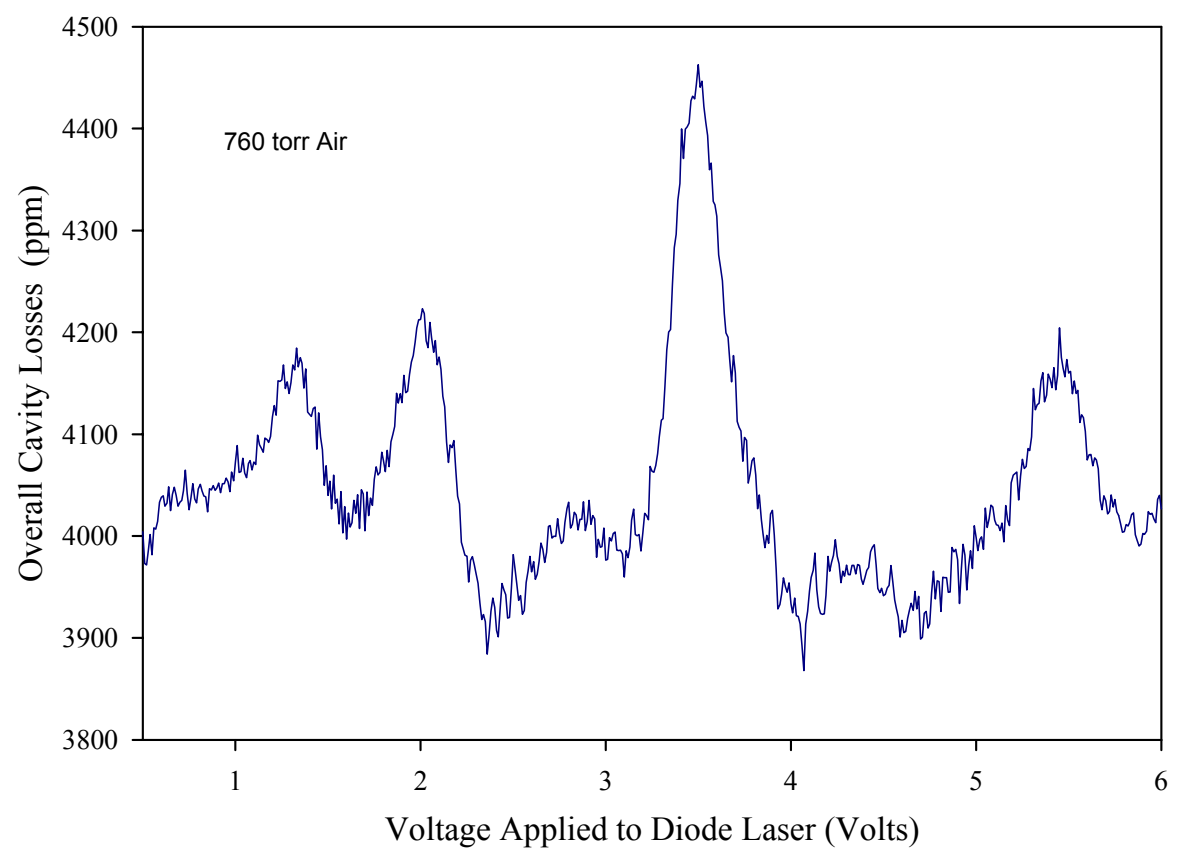

Figure 3: A portion of the $(7,0) \mathrm{A}-\mathrm{X}$ band of oxygen in the spectral region close to the $253.7 \mathrm{~nm}$ line of mercury. The scan range is about half of the total continuous range available with the diode seed laser or approximately $120 \mathrm{GHz}$ in the UV. 


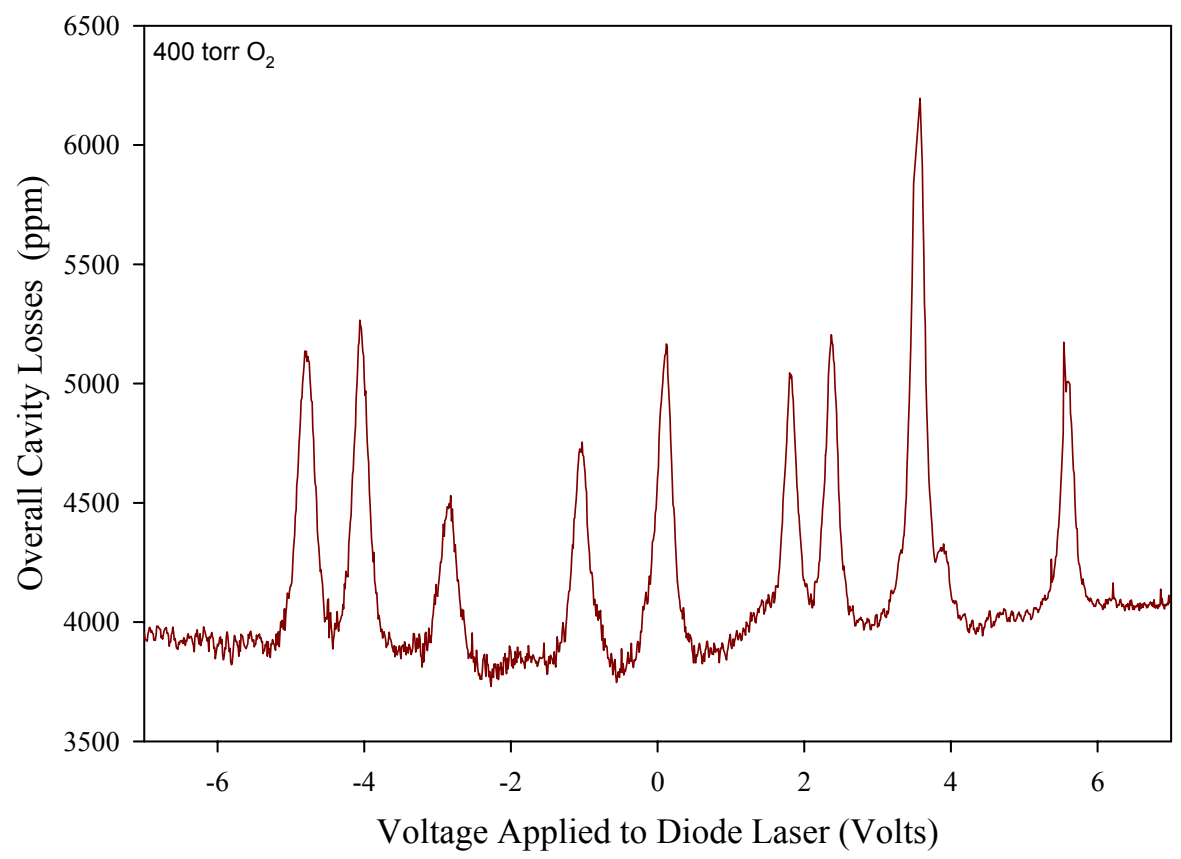

Figure 4: Scan of the seed diode laser voltage versus total losses with 400 torr of pure oxygen in the CRD cavity.

The continuous scan covered the entire $\sim 250 \mathrm{GHz}$ spectral region possible to scan with the diode seed laser in a single scan.

While taking data on the oxygen band shown above, the power and pointing stability of the laser system as well as the stability of the mirror alignment and coatings were monitored. While the power of the laser system did slowly decrease over time, due to a decrease in the pump lamp efficiency, this decrease did not effect the ring-down times or the results obtained. This is one advantage of cavity ring-down in that the intensity of the beam is not critical as only the rate of decay is measured to obtain data and not the intensity of the beam. The pointing stability of the laser system, as observed during this period, remained good and required very little adjustments. The mirrors were not taken out of the cavity or cleaned in any way for the entire time. No degradation in the baseline ring-down time of the system was observed over this time period. The alignment of the mirrors was not touched for a number of days at a time without any observable decrease in the baseline ring-down time.

The weak oxygen absorption provided a safe diagnostic tool to evaluate the scanning ability of the diode seed laser. This involved checking for hysteresis of scanning the laser both in the forward and backward direction. Also the reproducibility of scanning in one direction was checked. The latter seems quite reproducible when scanning in the same direction while the former shows a large hysteresis from a forward to a backward scan. Shown in Fig. 5 is a plot illustrating the difference between a forward and a backward voltage scan. A forward voltage scan was done from -7 volts to +7 volts while a backward scan was the reverse direction. In the plot shown in Fig. 5 the forward voltage scan is shown in dark red and the reverse voltage scan is shown in dark blue. From the figure it can be seen that the peak positions of the individual oxygen lines are shifted significantly in frequency, which is proportional to the applied voltage. However, a scan repeated in the same direction shows little or no significant frequency shift of the individual peaks. 


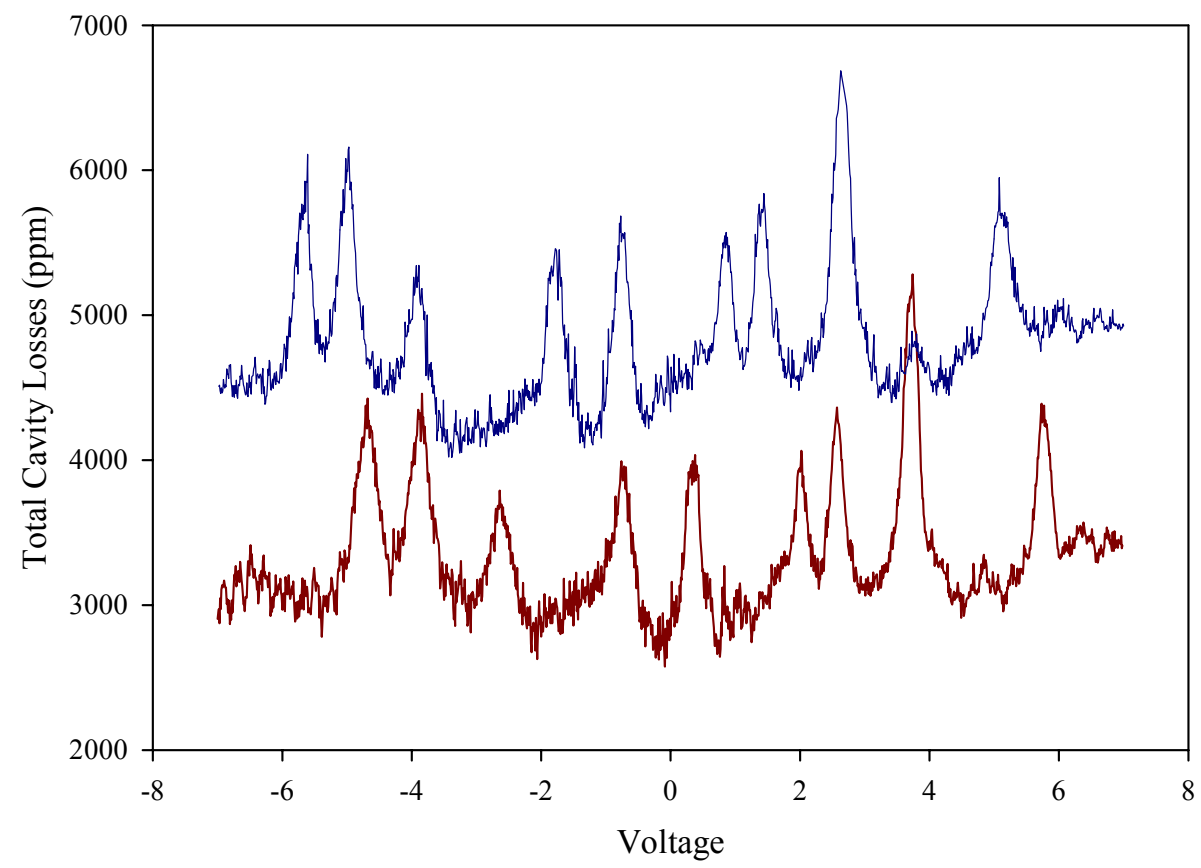

Figure 5: Scans over the $(7,0)$ A-X oxygen band. The bottom plot (red) is a forward voltage scan $(-7$ to +7 volts), while the top plot (blue) is a reverse scan of the voltage. The baseline level of the plots has been shifted for clarity. The applied voltage is directly proportional to the frequency output of the laser.

During this phase of the project a custom-built iodine cell was incorporated into the CRD setup for absolute wavelength calibration of the Alexandrite laser system. The iodine cell was an enclosed glass cylinder approximately $15 \mathrm{~cm}$ in length and $2.5 \mathrm{~cm}$ in diameter. A few crystals of iodine were placed in the cell; it was evacuated and then sealed. The molecular iodine atlas, in the spectral region of the fundamental wavelength of the Alexandrite laser, requires that the cell be heated to about $250^{\circ} \mathrm{C}$. The cell was heated to this temperature in an oven. The fundamental wavelength of the Alexandrite laser system, used to obtain the ultra-violet light needed for the mercury atomic transition, is in the region of $761 \mathrm{~nm}$. This beam was put through the cell and the absorption of light measured with a silicon photodiode (Thorlabs Inc., PDA155).

After a calibration of the Alexandrite system was performed the absolute quantum number assignment could be made for the previously observed transitions of oxygen. This weak, electronic dipole forbidden transition is shown below in Fig. 6. From the figure it can be seen that the transitions observed actually correspond to three different excited electronic states. These have been labeled in the figure. The absorption cross-section for these individual transitions ranges from $10^{-24}$ to $10^{-23} \mathrm{~cm}^{2}$. The cross section for these weak transitions can be compared to the atomic mercury absorption cross section which is an allowed transition and is on the order of $10^{-14} \mathrm{~cm}^{2}$ or $10^{10}$ times stronger than these observed oxygen transitions. 


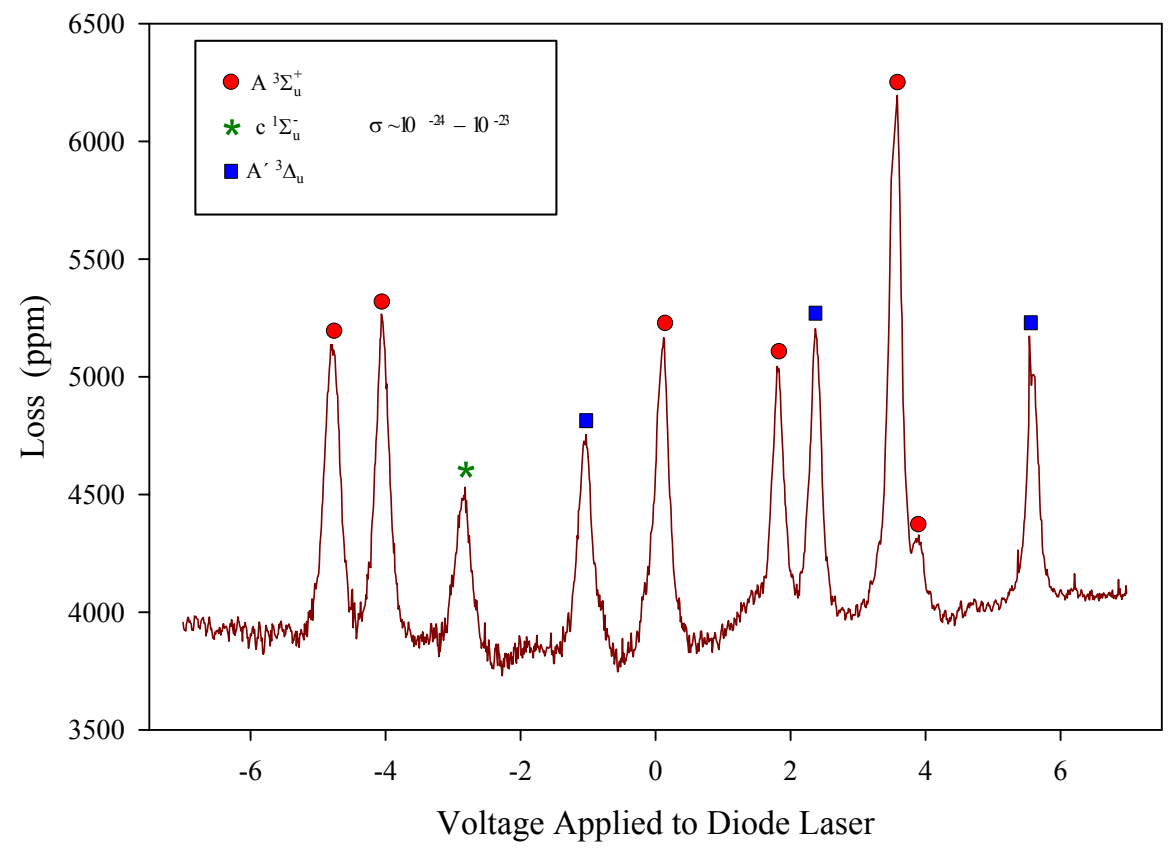

Figure 6: Scan of the oxygen transition originating from the ground $\mathrm{X}^{3} \Sigma_{\mathrm{g}}{ }^{-}$electronic state and terminating on one of 3 excited electronic states labeled on the plot. The absorption cross-section, $\sigma$, for the various transitions is given in $\mathrm{cm}^{2}$.

\section{Mercury Detection and Isotopic Structure}

After the system had been calibrated the Alexandrite laser was tuned to the spectral region around the $253.7 \mathrm{~nm}$ transition of atomic mercury. Mercury was sent to the cavity in known quantities using a temperature controlled mercury permeation tube delivery system. The amount of mercury entering the cell was verified using the atomic fluorescence spectrometer. This was done at the exit of the temperature controlled system as well as the entrance and exit of the CRD cavity to ensure that there were no mercury losses incurred in the delivery system.

The CRD cavity was kept at atmospheric pressure (760 torr) and the diode seed laser was scanned over the mercury transition. The resulting plot is shown in Fig. 7. The scale shown at the bottom of the plot is the voltage applied to the diode seed laser. The scale corresponds to approximately $18 \mathrm{GHz}$ per volt. The mercury concentration used was approximately 1.5 parts-per-billion by volume (ppb). From the plot it can be seen that there is more than one individual line that makes up the observed structure. This can be seen by the shoulders present in the spectrum as well as multiple peaks observed near the maximum of the observed peak. Atomic mercury has 7 naturally occurring isotopes. ${ }^{6}$ The individual isotopes should exhibit a slight wavelength shift relative to each other. The linewidth of the diode seeded Alexandrite laser system should be spectrally narrow enough $(\sim 180 \mathrm{MHz})$ to resolve the isotopic structure of mercury. Table 1 below lists all the naturally occurring isotopes of mercury. 


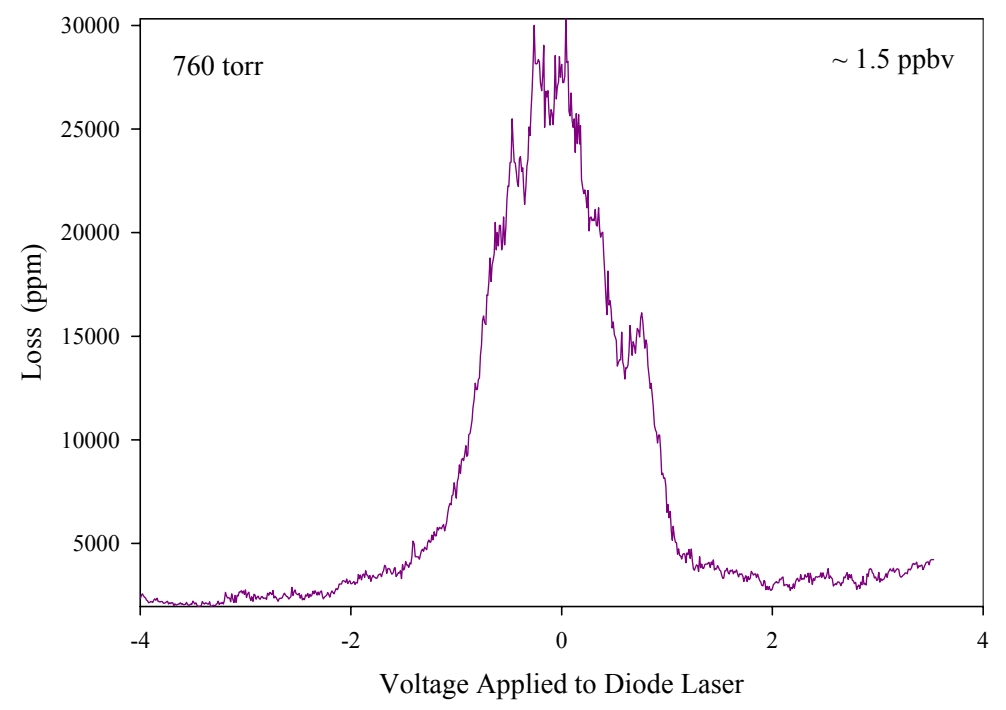

Figure 7: Scan of the $253.7 \mathrm{~nm}$ transition of atomic mercury. The pressure in the CRD cavity was 760 torr and the mercury concentration was about $1.5 \mathrm{ppb}$. The full-width half-maximum of the mercury line is approximately $22.5 \mathrm{GHz}$ due to the isotopic structure of mercury (see text) combined with pressure and Doppler broadening.

Included in Table 1 is the value of the nuclear spin for the odd isotopomers. The various isotopes each have individual spectral shifts. In addition, the hyperfine interaction causes the odd isotopomers to split as well as shift. Schweitzer was able to experimentally measure the individual isotopic shifts as well as the hyperfine splittings. ${ }^{7}$ Shown in Fig. 8 is a schematic plot of the isotopic shifts and hyperfine splitting for the various isotopes of mercury, as noted on the plot. Included on the top right side of the plot are a couple of lines indicating the spectral linewidth of a single isotopic line of mercury for 760 torr and 100 torr cavity pressure. For a pressure of 760 torr the linewidth is dominated by pressure broadening while at 100 torr both Doppler broadening and pressure broadening components are comparable. From the indicated linewidths we would not expect to resolve isotopic structure at 760 torr, as was observed in Fig. 7 , but at 100 torr we expect to resolve 5 distinct isotopic components. These 5 isotopic components have 2 that are due to individual isotopes and 3 that are a combination of transitions due to individual isotopes as well as hyperfine splitting components.

Indeed, if the pressure within the CRD cavity is decreased to 100 torr we are able to resolve these 5 lines due to the isotopic and hyperfine structure. A plot of the resulting scan is shown in Fig. 9. The concentration of mercury used for this particular scan is approximately 500 pptr. From this plot it can be seen that the detection limits for atomic mercury are in the low pptr range. The bars shown under each line are the calculated natural abundances for the five different lines. From the plot it can be seen that the observed intensity agrees very well with the predicted intensities from the naturally occurring isotopes of mercury. 


\begin{tabular}{|c|c|}
\hline Isotope & Natural Abundance (\%) \\
\hline 196 & $0.15(1)$ \\
\hline 198 & $9.97(8)$ \\
\hline $199(\mathrm{I}=1 / 2)$ & $16.87(10)$ \\
\hline 200 & $23.10(16)$ \\
\hline $201(\mathrm{I}=3 / 2)$ & $13.18(8)$ \\
\hline 202 & $29.86(20)$ \\
\hline 204 & $6.87(4)$ \\
\hline
\end{tabular}

Table 1: Isotopic abundances of all naturally occurring isotopes of mercury.

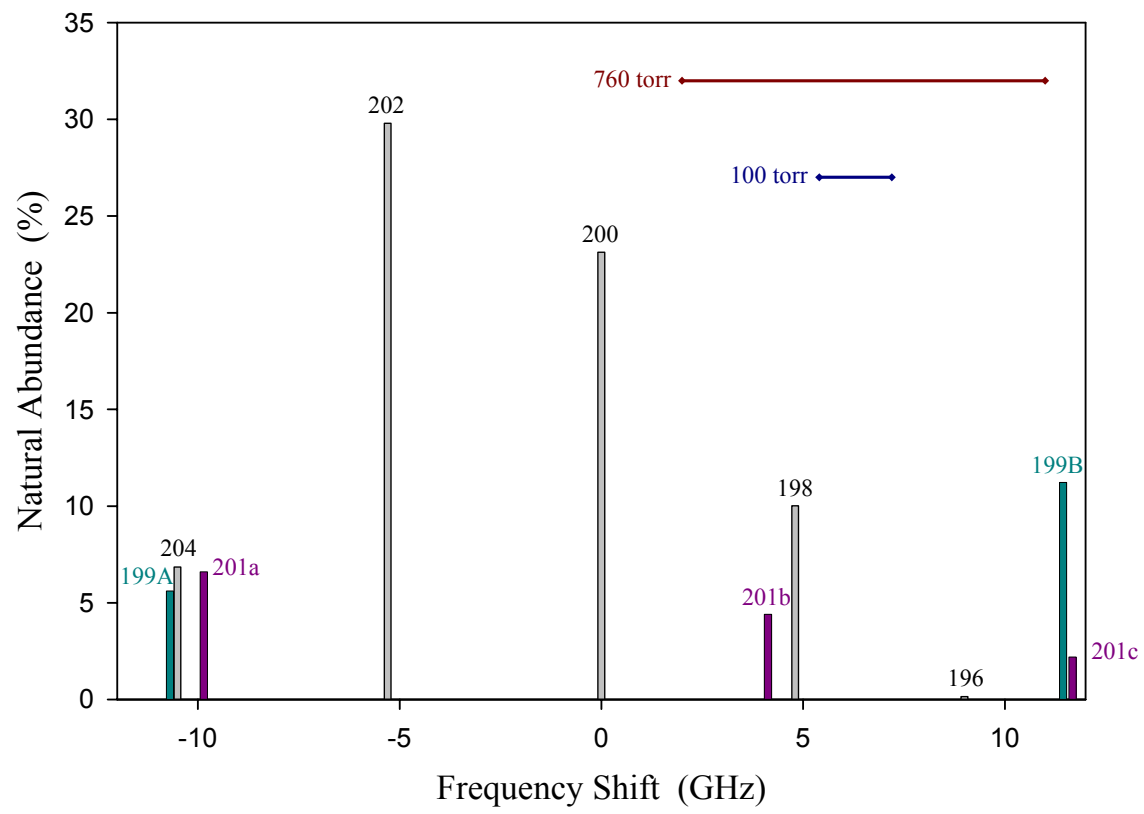

Figure 8: Schematic plot of the isotopic shifts and hyperfine splitting of the various isotopes of mercury. Also indicated in the plot is the expected linewidth for a single isotopomer line at 760 and 100 torr cavity pressure. 


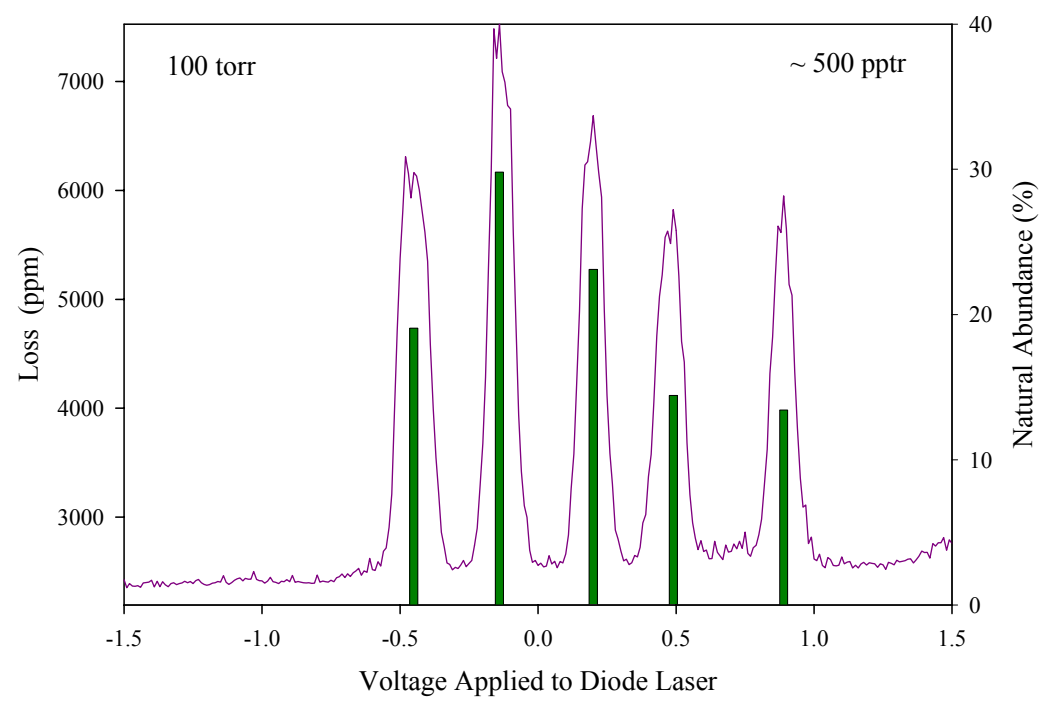

Figure 9: Scan of $\sim 500$ pptr of mercury at a cavity pressure of 100 torr showing the resolved isotopic structure of atomic mercury. The bars are the predicted natural abundances for each resolved isotopic peak. Due to the hyperfine splitting some of the peaks contain more than one single line resulting in 5 lines from 7 different isotopomers.

\section{Interferent Gas Testing}

With the detection limits of mercury down in the low pptr range in air the next step was to determine any background absorptions or interferences caused by other constituents of flue gas emissions from coalfired power plants. Table 2 lists the untreated flue gas components from a power plant burning low sulfur eastern bituminous coal. ${ }^{8}$ The total mercury concentration listed is in the low ppb range, which is well above our present detection limits. The majority of the other components of flue gas are in the ppm range or higher meaning that their concentrations are in excess of 1000 times the concentration of mercury. From the list the component of greatest concern, in terms of an UV absorption technique, is $\mathrm{SO}_{2}$. Sulfur dioxide is known to absorb in the same UV region as mercury.

\section{Individual Coal-Fired Flue Gas Components}

The number and components of flue gas present a very challenging environment for the analysis of mercury. Most components of flue gas are in far greater abundance than mercury and a number of them can cause interferences with equipment attempting to determine the amount of mercury contained within the flue gas stream. As a result, the majority of mercury Continuous Emission Monitors (CEMs) require a certain degree of sample pretreatment. This pretreatment can be as extreme as removing everything from the gas stream except the mercury prior to sampling.

Given below in Table 2 is a list of components, and their concentrations, observed from untreated flue gas from a coal-fired power plant burning eastern bituminous coal. ${ }^{8}$ SRD scientists have individually tested the majority of species shown in Table 2 . The species tested include sulfur dioxide $\left(\mathrm{SO}_{2}\right)$, carbon dioxide 
$\left(\mathrm{CO}_{2}\right)$, carbon monoxide $(\mathrm{CO})$, nitrogen dioxide $\left(\mathrm{NO}_{2}\right)$, nitric oxide $(\mathrm{NO})$, hydrogen chloride $(\mathrm{HCl})$, oxygen $\left(\mathrm{O}_{2}\right)$, nitrogen $\left(\mathrm{N}_{2}\right)$, and water $\left(\mathrm{H}_{2} \mathrm{O}\right)$. Sulfur dioxide has a broad essentially structureless absorption in the same spectral region as mercury, which presents a problem for most mercury CEM detectors. The effects and work done on $\mathrm{SO}_{2}$ will be detailed in the next section. Concentrations tested for the other components of flue gas were generally much higher than that found in the untreated flue gas shown in Table 2. Oxygen, $\mathrm{N}_{2}$, and $\mathrm{CO}_{2}$ have been tested at or near $100 \%$. The only species given in the table that have not been individually tested are $\mathrm{SO}_{3}$ and hydrocarbons. The concentrations of $\mathrm{SO}_{3}$ and the various hydrocarbons present in the untreated flue gas are both quite low. Hydrocarbons are not known to absorb in the same UV region as mercury and were therefore not specifically tested. Sulfur trioxide, aside from being present in low concentration, is difficult to generate and can be very reactive with air and hence it was also not tested.

\begin{tabular}{|c|c|}
\hline Species & Volume \\
\hline $\mathrm{H}_{2} \mathrm{O}$ & $5-7 \%$ \\
\hline $\mathrm{O}_{2}$ & $3-4 \%$ \\
\hline $\mathrm{CO}_{2}$ & $15-16 \%$ \\
\hline $\mathrm{Total} \mathrm{Hg}$ & $1 \mathrm{ppb}$ \\
\hline $\mathrm{CO}$ & $20 \mathrm{ppm}$ \\
\hline $\mathrm{Hydrocarbons}$ & $10 \mathrm{ppm}$ \\
\hline $\mathrm{HCl}$ & $100 \mathrm{ppm}$ \\
\hline $\mathrm{SO}_{2}$ & $800 \mathrm{ppm}$ \\
\hline $\mathrm{SO}_{3}$ & $10 \mathrm{ppm}$ \\
\hline $\mathrm{NO}_{\mathrm{x}}$ & $500 \mathrm{ppm}$ \\
\hline $\mathrm{N}_{2}$ & Balance \\
\hline
\end{tabular}

Table 2: Typical untreated flue gas composition from a coal-fired power plant burning low sulfur eastern bituminous coal. ${ }^{8}$

Nitric oxide and nitrogen dioxide were both individually tested up to $350 \mathrm{ppm}$. An example of this can be seen in Fig. 10. In this plot a scan of just mercury in air is shown by the blue plot while a scan with mercury, $350 \mathrm{ppm}$ NO, and the balance air is shown by the red plot. The concentration of mercury used was $3.5 \mathrm{ppb}$, the cell was held at about 120 torr pressure, and maintained at a temperature of $200^{\circ} \mathrm{C}$. From the plot it can be seen that the two data traces overlap each other almost exactly, with only a slight deviation on a peak or two. Also it should be noted that the background levels of the two plots are essentially equal indicating no additional background absorptions by NO. The results were identical for $\mathrm{NO}_{2}$. There was no observable absorption due to $\mathrm{NO}_{2}$ in the same UV region of the mercury transition. 
Water is known to have some weak absorptions in the UV although most likely not in the region of the mercury transition. However SRD did perform tests to confirm this assertion. The level of water in the sample gas stream was increased by passing dry nitrogen through a water bubbler, at room temperature, just prior to the entrance to the sample cavity. An example of this can be seen in Fig. 11. Here again the blue plot shows a scan across the mercury transition for data taken with just mercury and air in the sample gas stream. The red plot is from data taken with mercury, an elevated water level, and the balance air. This data was taken with a mercury concentration of $2.7 \mathrm{ppb}$, a cavity pressure of 400 torr, and the temperature was maintained at $200^{\circ} \mathrm{C}$. From the plot it can be seen that the two traces are nearly identical indicating no additional absorption due to elevated levels of water in the sample gas stream.

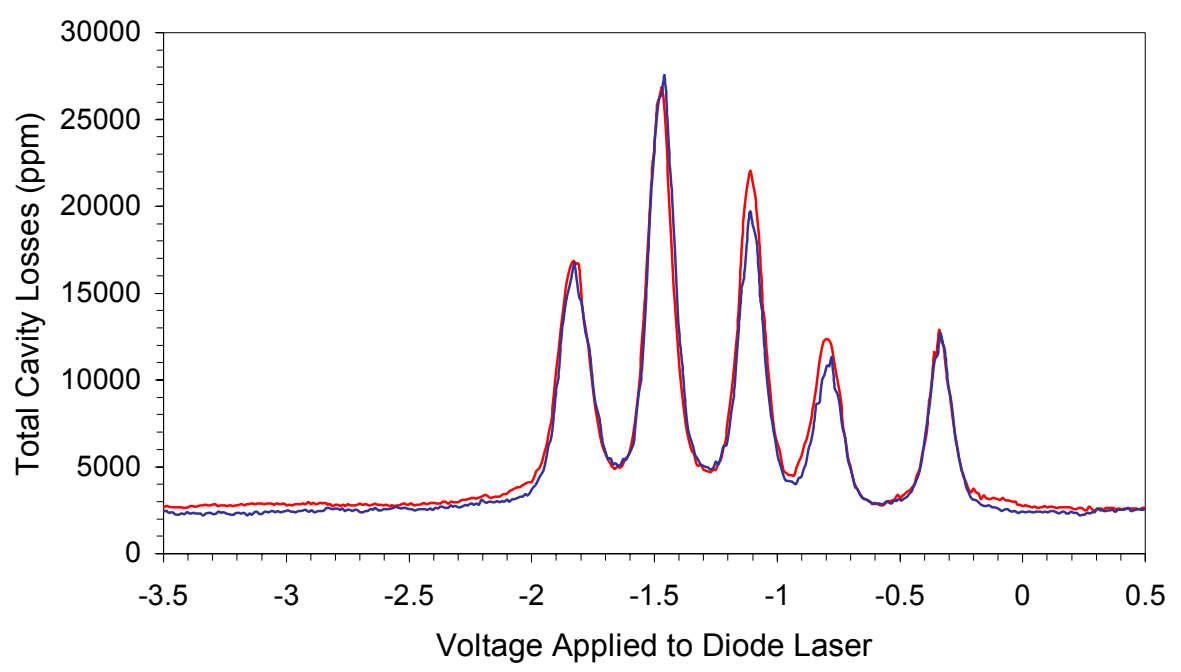

Figure 10: Plot of two scans over the mercury transition at $253.7 \mathrm{~nm}$. The plot shown in blue is data take with 3.5 ppb mercury and the balance air. The plot shown in red is data taken with 3.5 ppb mercury, 350 ppm NO, and the balance air.

Carbon monoxide was tested at concentrations up to $285 \mathrm{ppm}$. The results obtained were similar to the nitrogen oxides and water. There was no observable effect on the ring-down signal and therefore no measurable absorption due to CO. Carbon dioxide was tested up to a concentration of about $1400 \mathrm{ppm}$ with no changes in the cavity losses observed. Both $\mathrm{O}_{2}$ and $\mathrm{N}_{2}$ were tested up to $100 \%$ concentration. Here the results were similar to those obtained for the species mentioned already. There was no measurable absorption for either species. Each of the above mentioned species was tested individually to observe any effect on the losses within the cavity in the presence of about 1-4 ppb of mercury to ensure the proper concentration of mercury was reported in the presence of the various interferents. Some of the species were also tested with no mercury in the sample gas to ensure the background level remained the same. It was found that there was no significant effect on the background loss level of the cavity observed for any of the species mentioned above. In addition the concentration of mercury could be reproducibly determined in the presence of any of the individually tested interferents.

The results obtained above were very positive and showed good promise for the ability of the CRD instrument to determine the concentration on mercury within the complex matrix of flue gas from coalfired power plants. However, the concentration of $\mathrm{CO}_{2}$ initially tested was quite a bit lower than that found in the untreated flue gas and $\mathrm{HCl}$ needed to be tested. SRD scientists did test both of these flue gas components at concentration levels greater than those shown in Table 2. 


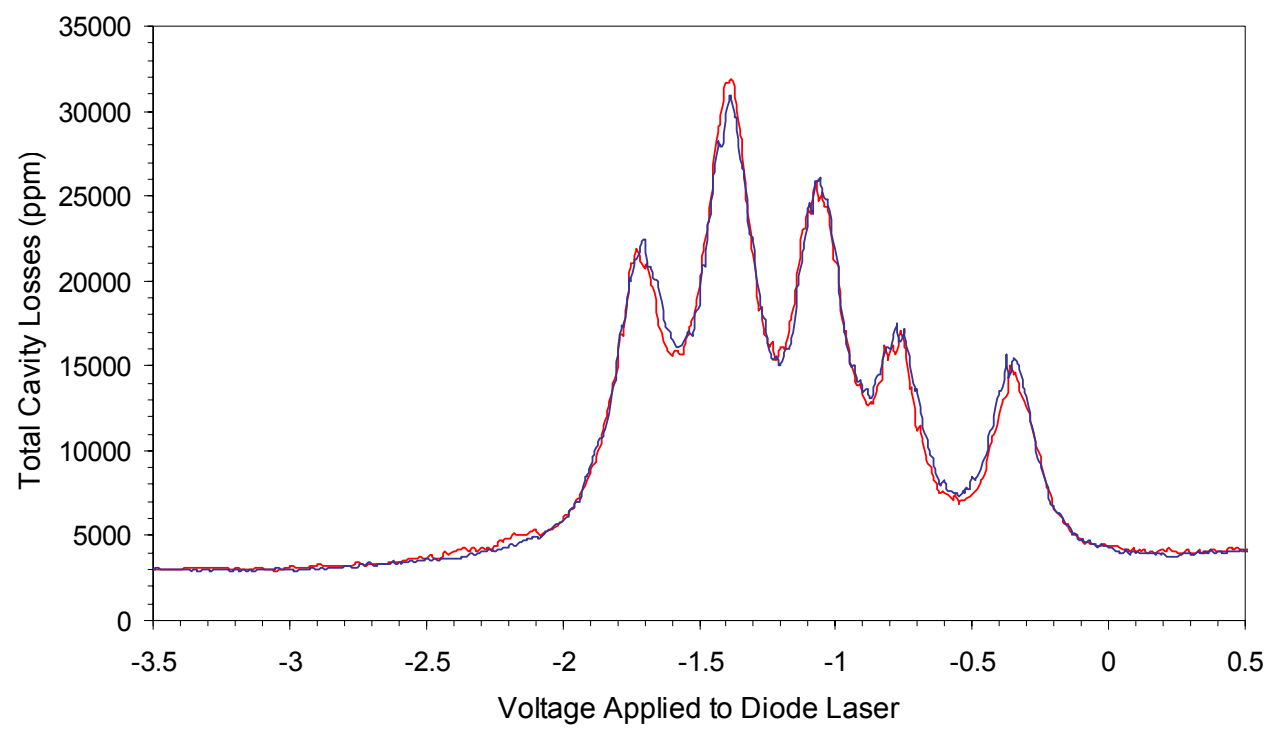

Figure 11: Plot of two scans over the mercury transition at $253.7 \mathrm{~nm}$. The plot shown in blue is data take with 2.7 ppb mercury and the balance air. The plot shown in red is data taken with 2.7 ppb mercury, elevated concentration of $\mathrm{H}_{2} \mathrm{O}$ from a bubbler, and the balance air.

Figure 12 shows a scan of the diode laser, used to seed the Alexandrite laser, over the $253.7 \mathrm{~nm}$ region of the UV in the area of the mercury transition. The concentration of mercury used during this scan was approximately $4.5 \mathrm{ppb}$ in air, the temperature of the cell was room temperature, and the pressure of the cell was held at 200 torr. From the figure the 5 different isotopic peaks defining the mercury transition can be clearly observed. There is a very high signal-to-noise level and the baseline cavity loss level is slightly above $3800 \mathrm{ppm}$. The blue data in the plot was obtained from the CRD ring-down signal while the red data is a plot of the theoretical calculation for the experimental parameters used in obtaining the data.

Depicted in Fig. 13 is a scan over the identical UV region of the spectrum containing the mercury transition. The data taken for this plot was done with a sample gas stream of air only. The conditions used for the scan were that the cavity was held at room temperature and the pressure was approximately atmospheric pressure. From the plot it can be seen that there is no observable absorption due to mercury. It was also determined that the baseline cavity loss level observed for the scan was approximately 3800 ppm. The baseline loss level observed for the scan in air shown in Fig. 13 is essentially identical to the baseline loss level observed for the scan of mercury in air depicted in Fig. 12.

After establishing the baseline loss level a sample mixture of $400 \mathrm{ppm} \mathrm{HCl}$ in air was sent through the ring-down cavity. The results of the scan over the same UV region are shown in Fig. 14. From the figure it can be seen that the baseline is flat over the entire region of the scan. In addition, the level of total cavity losses remained at $3800 \mathrm{ppm}$ showing that there are no observable losses due to $\mathrm{HCl}$, at least up to $400 \mathrm{ppm}$ concentration, within the UV region of the spectrum covering the mercury transition. 


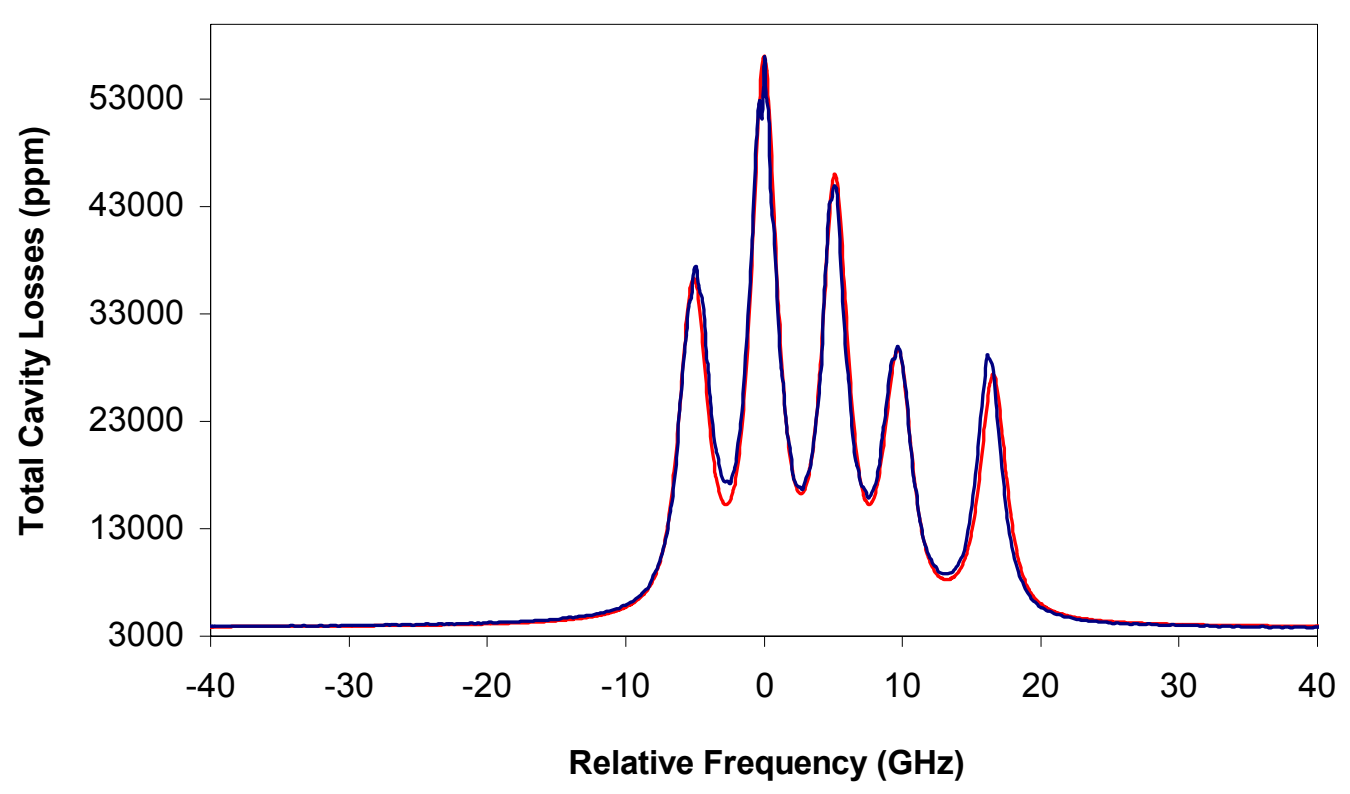

Figure 12: Scan over the $253.7 \mathrm{~nm}$ region containing the mercury transition. The mercury concentration was approximately $4.5 \mathrm{ppb}$ in air. The blue plot is the ring-down data obtained and the red plot is a theoretical prediction for the experimental conditions used.

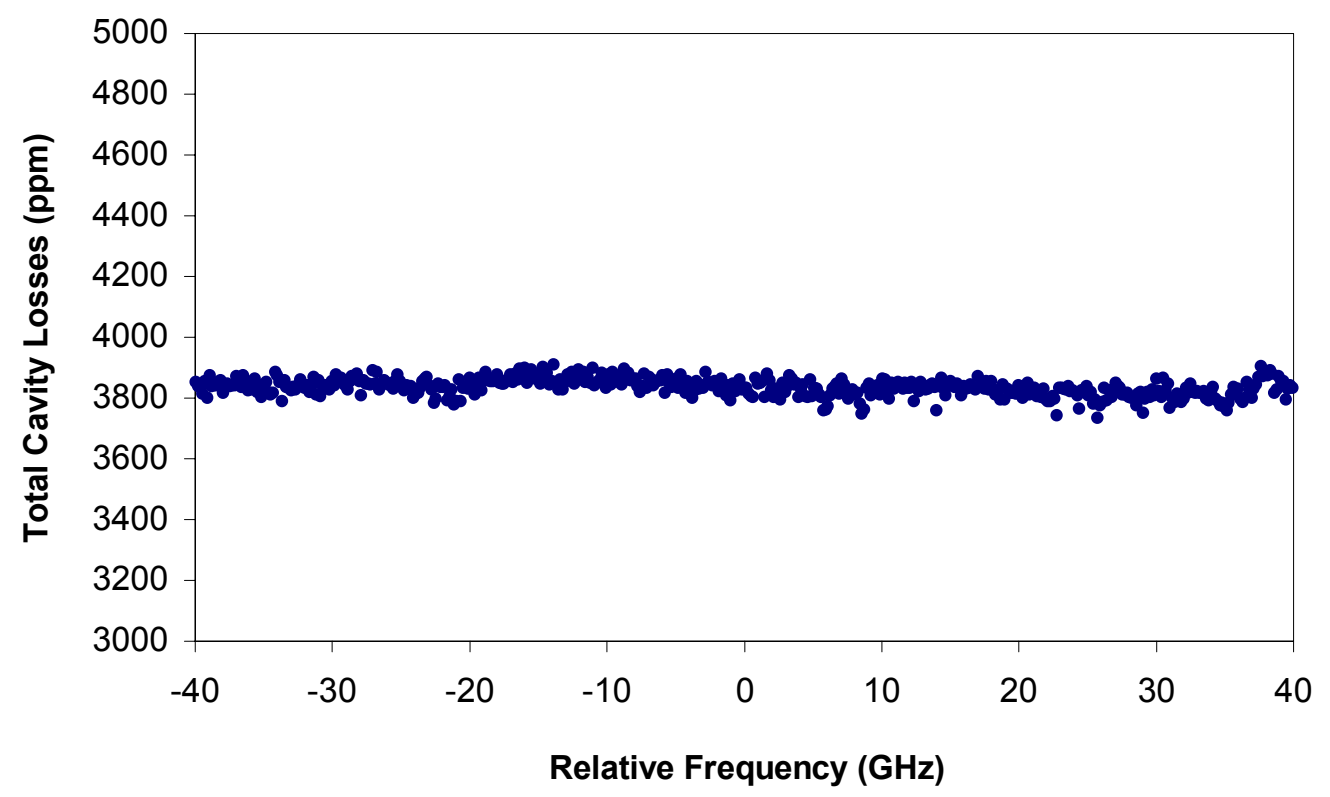

Figure 13: Scan over the UV region containing the mercury transition. The sample gas flowing through the cavity contained only air. 


\section{Sensor Research \& Development Corporation DE-FC26-01FT41221}

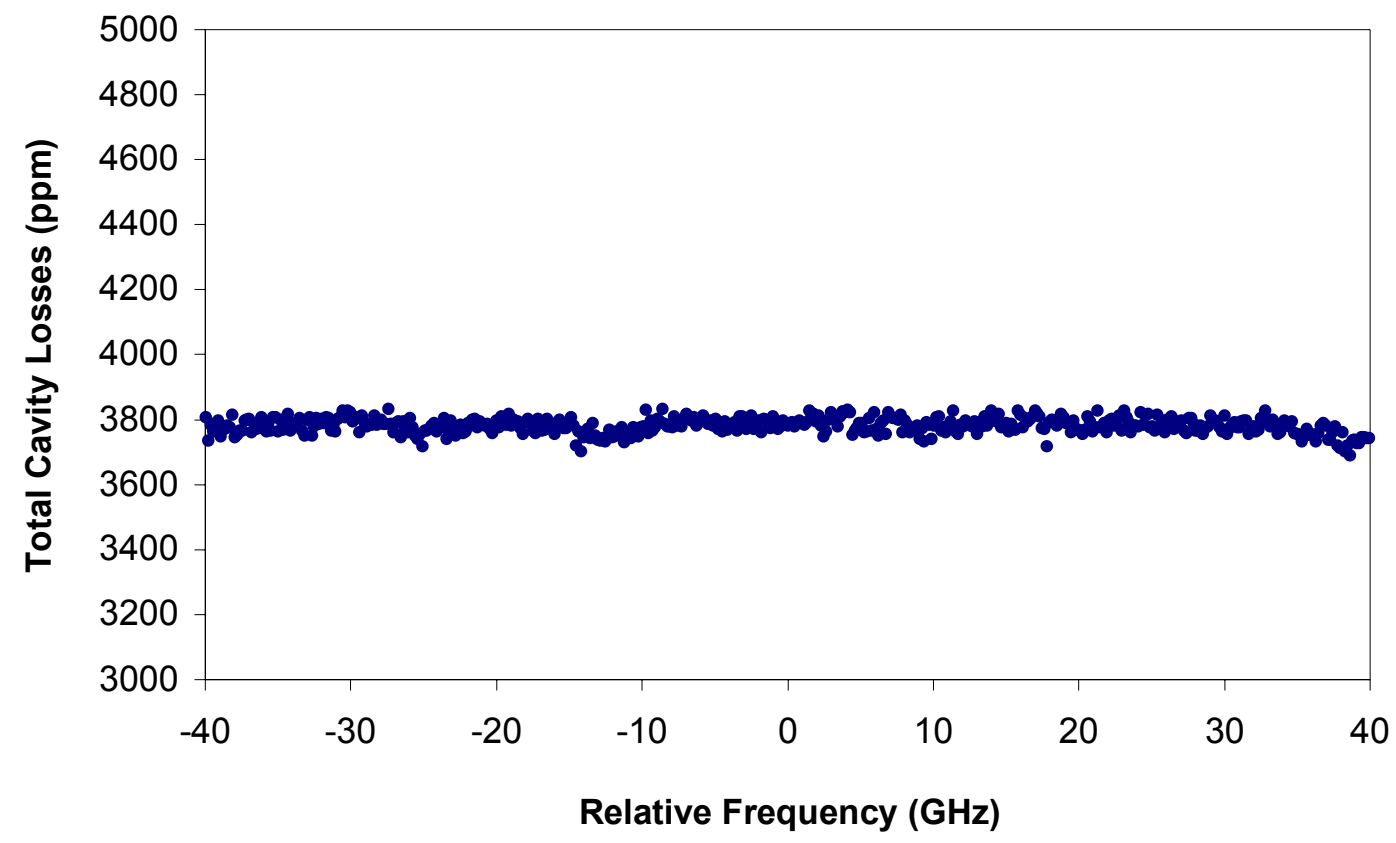

Figure 14: Scan over the UV region containing the mercury transition. The sample gas contained $400 \mathrm{ppm}$ of $\mathrm{HCl}$ with the balance air.

The results of tests, done with a $100 \%$ concentration of $\mathrm{CO}_{2}$, are shown in Fig. 15 below. From the figure it can be seen that the baseline level is flat and that there are no discrete transitions due to $\mathrm{CO}_{2}$ contained within the same UV region as the mercury transition. However, it was observed that the baseline loss level of the cavity did increase very slightly. There was about a 5\% increase in the total cavity loss level to about $4000 \mathrm{ppm}$. This result was confirmed with multiple scans of both $\mathrm{CO}_{2}$ and air. The most likely cause of this increase in loss level is due to scattering losses. Rayleigh scattering of the UV radiation in $\mathrm{CO}_{2}$ must be somewhat larger than in air. While there was a slight increase in the cavity losses observed it should be noted that this small change would not result in a change of the ability of the CRD system to quantify the amount of mercury in a sample gas stream. In addition, the stack emissions to be measured from any coal-fired power plant would not be close to $100 \% \mathrm{CO}_{2}$ so this effect will be even less than that observed during this test and, as noted previously, at lower $\mathrm{CO}_{2}$ concentrations there was no observable change in the baseline losses of the cavity.

SRD scientists have tested, with the exception of $\mathrm{SO}_{3}$ and the low level of hydrocarbons, all of the constituents of the untreated flue gas shown in Table 2. The concentration levels tested of the various species were in excess of those found in the untreated flue gas. The only species, other than $\mathrm{SO}_{2}$, observed that had any effect on the total losses within the ring-down cavity, in the same UV region that contains the mercury transition, was $\mathrm{CO}_{2}$ at a concentration level of $100 \%$. The effects due to $\mathrm{CO}_{2}$ mentioned above are extremely small, even with a concentration of $100 \%$, and cause no significant effect on the CRD instruments ability to determine mercury concentrations. 


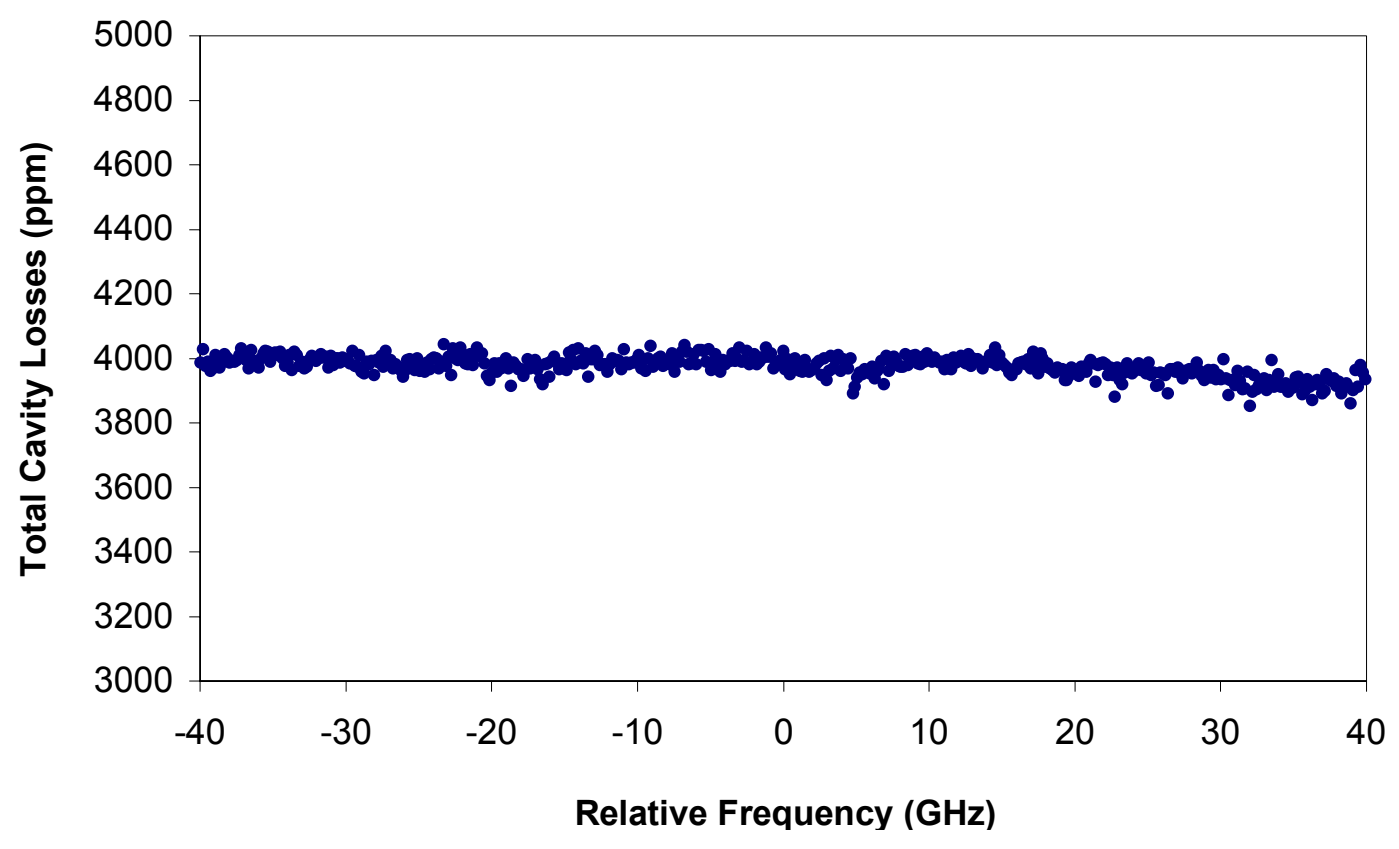

Figure 15: Scan over the $253.7 \mathrm{~nm}$ region containing the mercury transition. The sample gas flowing through the ring-down cavity contained $100 \% \mathrm{CO}_{2}$.

\section{Sulfur Dioxide}

Sulfur Dioxide is known to absorb in the same region of the ultra-violet as mercury. The absorption crosssection for $\mathrm{SO}_{2}$ has been experimentally determined by a number of people ${ }^{9-12}$ to be on the order of $10^{-19}$ $\mathrm{cm}^{2}$. While this cross-section is 5 orders of magnitude smaller than that of mercury, the concentration of $\mathrm{SO}_{2}$ in flue gas, from Table 2, is approximately 5-6 orders of magnitude higher than typical levels of mercury. Indeed, presently used commercial CEMs based on atomic emission, atomic fluorescence, or atomic absorption require the removal of $\mathrm{SO}_{2}$, and other flue gas constituents, before an accurate determination of the mercury concentration can be made.

The addition of $\mathrm{SO}_{2}$ to the CRD cavity has an immediate effect on the baseline loss level of the CRD instrument. There is an observable increase in the base absorption losses of the cavity, dependent on the $\mathrm{SO}_{2}$ concentration. However, the absorption loss level due to $\mathrm{SO}_{2}$ is constant over the frequency range used to observe the mercury transitions. The absorption is also unstructured indicating that there would be no possibility of observing a peak of $\mathrm{SO}_{2}$ rather than mercury. This can be seen in Fig. 16. This figure contains two scans over the region containing the mercury transition. The data shown in blue is the baseline level obtained with only air in the sample gas stream. The orange plot is the baseline level obtained with $1000 \mathrm{ppm} \mathrm{SO}$ and a balance of air in the sample gas. Notice the large change in baseline level due to the absorption of the UV radiation by $\mathrm{SO}_{2}$. 


\section{Sensor Research \& Development Corporation DE-FC26-01FT41221}

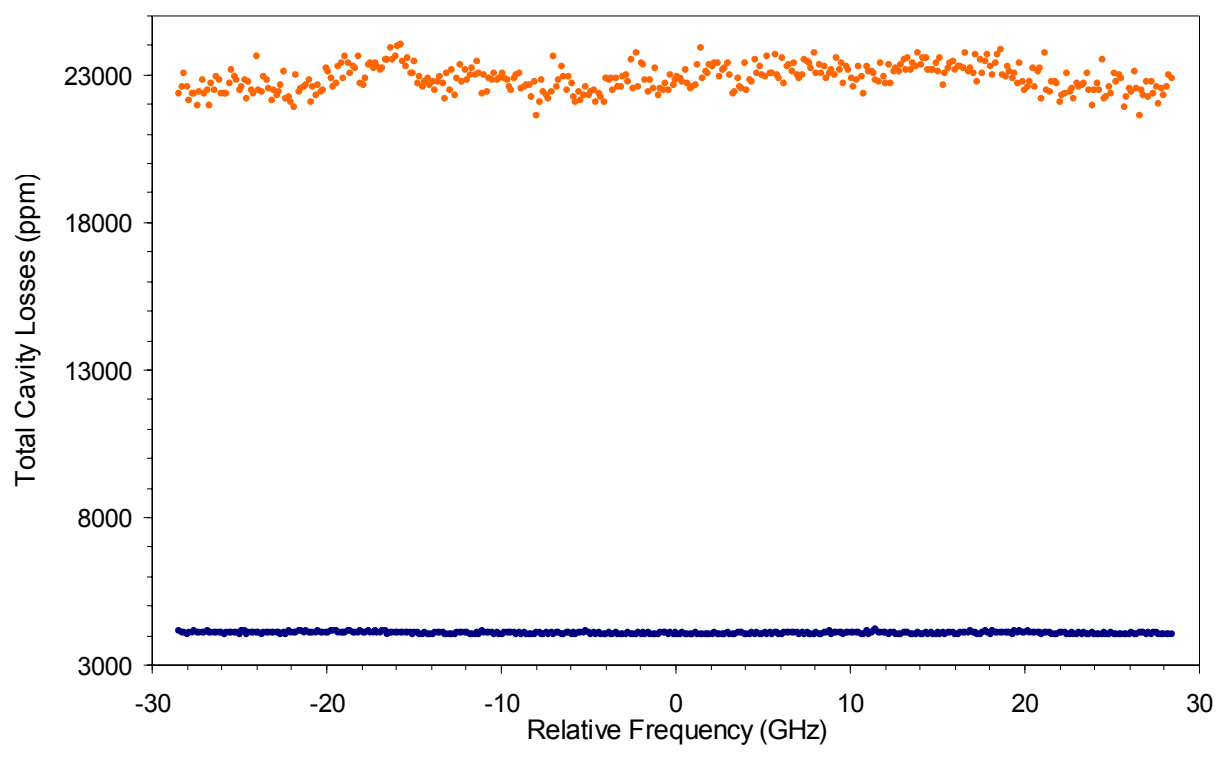

Figure 16: Plot of the frequency region around the $253.7 \mathrm{~nm}$ transition of mercury. The blue plot is the baseline level with only air flowing through the cavity and the orange plot is the baseline cavity losses with $1000 \mathrm{ppm}$ $\mathrm{SO}_{2}$ with a balance of air.

In an effort to incorporate $\mathrm{SO}_{2}$ concentrations into the theoretical model, $\mathrm{SRD}$ performed some additional tests. Shown in Fig. 17 is a plot of the absorption losses versus concentration of $\mathrm{SO}_{2}$. The point on the left side of the plot, shown in Fig. 17 at 0 ppm, corresponds to baseline losses of an empty cavity with no $\mathrm{SO}_{2}$ present in the cell. The rest of the points correspond to concentrations ranging from 100 to $2500 \mathrm{ppm}$. This range of concentrations is generally greater than the output from coal-fired power plants. The higher concentrations tested were about 1 million times the concentration levels of mercury to be detected (low $\mathrm{ppb})$.

It is encouraging that the plot of losses versus concentration is linear. This is a good indication that we are not observing any saturation effects or non-Beer-Lambert law behavior, both of which could substantially inhibit the ability of the CRD instrument to accurately determine mercury concentrations with relatively high background absorptions. It should also be noted that the exponential decays used to determine the absorption due to $\mathrm{SO}_{2}$, while the time constant decreased, was still fit very well to a single exponential. Again this is also important for accurate determination of the mercury concentration.

From the information obtained, to produce the plot for Fig. 17, SRD was able to experimentally determine the absorption cross-section of $\mathrm{SO}_{2}$ in the wavelength region covering the UV mercury transition. The concentration of $\mathrm{SO}_{2}$ was determined from a certified cylinder and mass flow controllers, which were used to accurately measure the gas flow. SRD independently checked the concentration of $\mathrm{SO}_{2}$ from the cylinder using a gas chromatograph and the results agreed with the certified value. The experimentally determined value of the absorption cross-section of $\mathrm{SO}_{2}$ by $\mathrm{SRD}$ was approximately $1.6 \times 10^{-19} \mathrm{~cm}^{2}$. This value agrees quite well with the average experimentally determined value by a number of authors. ${ }^{9-12}$ This average determined value for the $\mathrm{SO}_{2}$ cross-section was found to be about $1.5 \times 10^{-19} \mathrm{~cm}^{2}$. 


\section{Sensor Research \& Development Corporation DE-FC26-01FT41221}

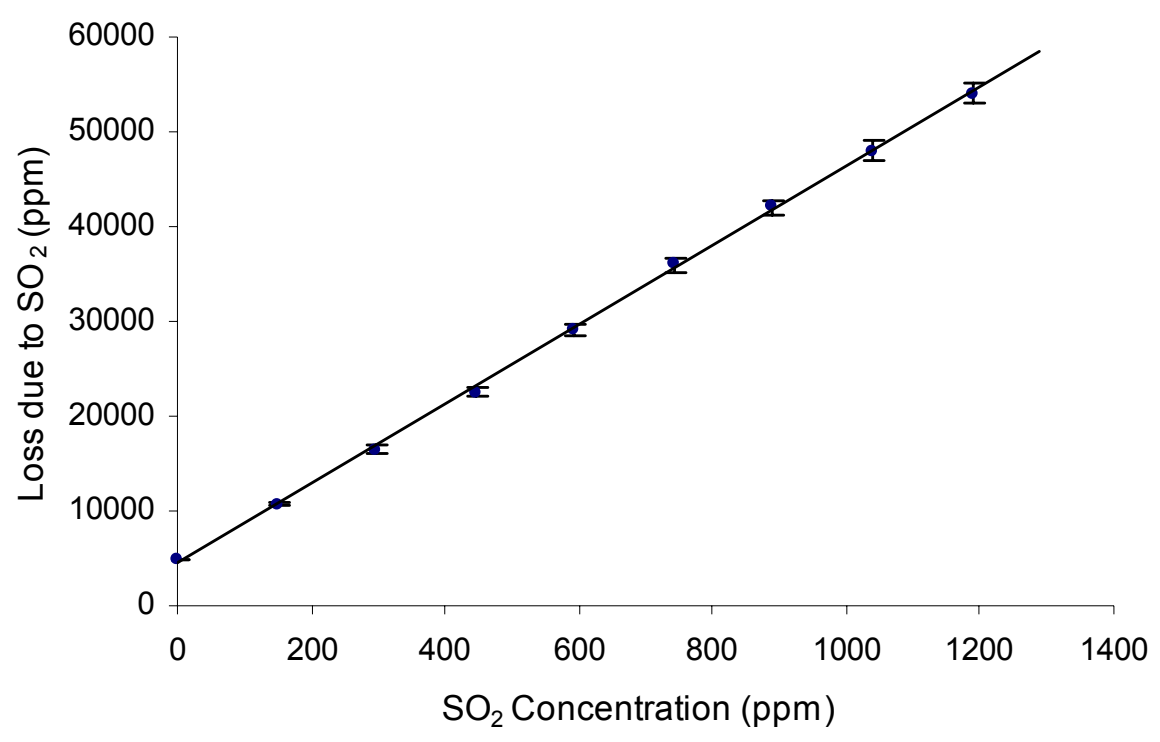

Figure 17: Baseline absorption losses versus $\mathrm{SO}_{2}$ concentration. From this plot the absorption cross-section for $\mathrm{SO}_{2}$ can be determined.

This experimentally determined cross-section has been incorporated into the theoretical model, which can now be used to determine both the mercury concentration and the $\mathrm{SO}_{2}$ concentration simultaneously (see section on Theoretical Modeling). This assumes that the losses in the cavity are due to only mercury, $\mathrm{SO}_{2}$, and the high reflectivity mirrors.

The next step was to actually detect mercury in the presence of $\mathrm{SO}_{2}$. Shown in Fig. 18 is a plot of the mercury isotopic structure observed in the presence of $\mathrm{SO}_{2}$. This scan was done with the $65 \mathrm{~cm}$ cavity held at a pressure of 100 torr and a temperature of $200^{\circ} \mathrm{C}$. The concentration of mercury used for this scan was approximately $1.2 \mathrm{ppb}$ while the concentration of $\mathrm{SO}_{2}$ used was about $2175 \mathrm{ppm}$. This is a difference in concentration of close to 2 million times. While the background absorption level is higher than previously observed with no $\mathrm{SO}_{2}$, from the plot it can be seen that the CRD instrument was able to easily detect mercury. The amount of mercury in the sample gas stream was close to the same concentration level as that found in the untreated flue gas, but the mercury was detected in the presence of $\mathrm{SO}_{2}$ at a concentration almost 3 times that reported for the particular untreated flue gas given in Table 2 . These results are very promising for the development of the CRD spectrometer without the need for extensive sample pretreatment as is needed for most mercury CEM monitors on the market today.

Figure 18 includes both the experimental plot, shown in blue, and the theoretically predicted mercury spectrum, shown in red. The calculated spectrum was done as described in the section, Theoretical Modeling, with an addition to the baseline losses to include losses from $\mathrm{SO}_{2}$. From the figure it can be seen that the agreement between the experimental and theoretically predicted results is very good. The limit of detection of mercury, in the presence of $\mathrm{SO}_{2}$, is dependent on the $\mathrm{SO}_{2}$ concentration. For example, an $\mathrm{SO}_{2}$ concentration of $1000 \mathrm{ppm}$, above that for the untreated flue gas given in Table 2, the limit of detection for mercury was determined to be about 150 pptr. This limit of detection is far below the mercury concentration expected in the flue gas stream. 


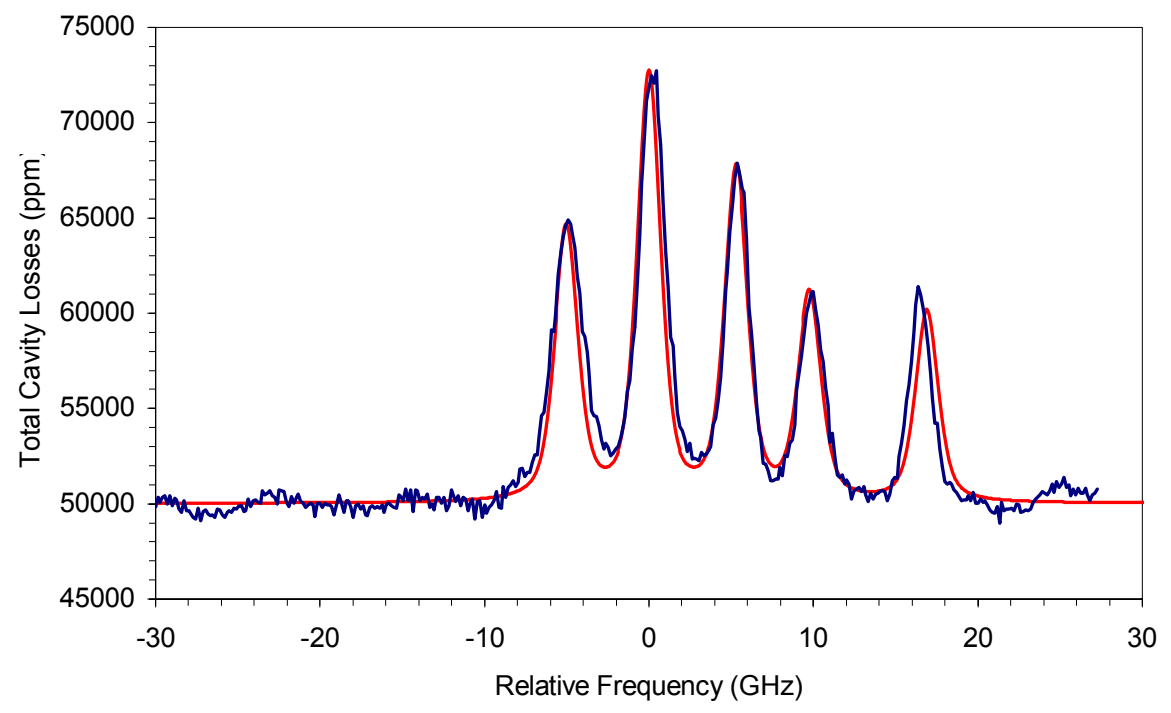

Figure 18: A plot of the losses in the $65 \mathrm{~cm}$ cavity versus relative frequency. The cavity was held at a pressure of 100 torr and a temperature of $200^{\circ} \mathrm{C}$. Clearly seen are the 5 mercury peaks above the $\mathrm{SO}_{2}$ background absorption. The concentration of mercury is $\sim 1.2 \mathrm{ppb}$ while that for $\mathrm{SO}_{2}$ is about $2175 \mathrm{ppm}$. The plot includes the experimental data (blue), and the theoretical plot (red) including both the mercury and $\mathrm{SO}_{2}$ concentrations, and losses from the mirrors.

Shown in Fig. 18 is the detection of mercury within a matrix of $\mathrm{SO}_{2}$ with the original $65 \mathrm{~cm}$ cell. As this $65 \mathrm{~cm}$ cavity had been replaced with a $40 \mathrm{~cm}$ cavity the detection of mercury within $\mathrm{SO}_{2}$ needed to be verified in the smaller cavity. Figure 19 is a plot of cavity losses versus relative frequency using the 40 $\mathrm{cm}$ cavity. The data shown in the plot was taken with a sample flow through the cavity of $\sim 1700 \mathrm{ppm} \mathrm{SO}_{2}$ and $\sim 1.5 \mathrm{ppb}$ mercury. This corresponds to a mercury concentration over 1,000,000 times less than $\mathrm{SO}_{2}$. The cell was maintained at a pressure of 50 torr, to fully resolve the individual mercury isotopic peaks, and the temperature was held at $200^{\circ} \mathrm{C}$. From the plot it can be seen that the baseline noise level, with a background of $\mathrm{SO}_{2}$, is somewhat, but not significantly, noisier than that observed with an empty cavity. It can also be seen that the CRD instrument was able to detect the low concentration level of mercury with a relatively good signal to noise ratio in the shorter $40 \mathrm{~cm}$ cavity. From the observed signal it can be seen that the detection limits for mercury are clearly at the sub-ppb level in the presence of these elevated concentrations of sulfur dioxide.

In addition to the experimental data shown in Fig. 19, there is also a theoretical plot included. The theoretical plot includes losses due to the mirrors, a specific concentration of mercury, and a specific concentration of $\mathrm{SO}_{2}$. From the figure it can be seen that the theoretically determined plot agrees extremely well with the experimental data. This gives us additional confidence in our ability to determine accurate concentrations of mercury even in the presence of strongly absorbing species, which raise the baseline level of the background. If the background absorption is due primarily to $\mathrm{SO}_{2}$ the CRD instrument can also determine the concentration of $\mathrm{SO}_{2}$ at the same time as the mercury concentration. 


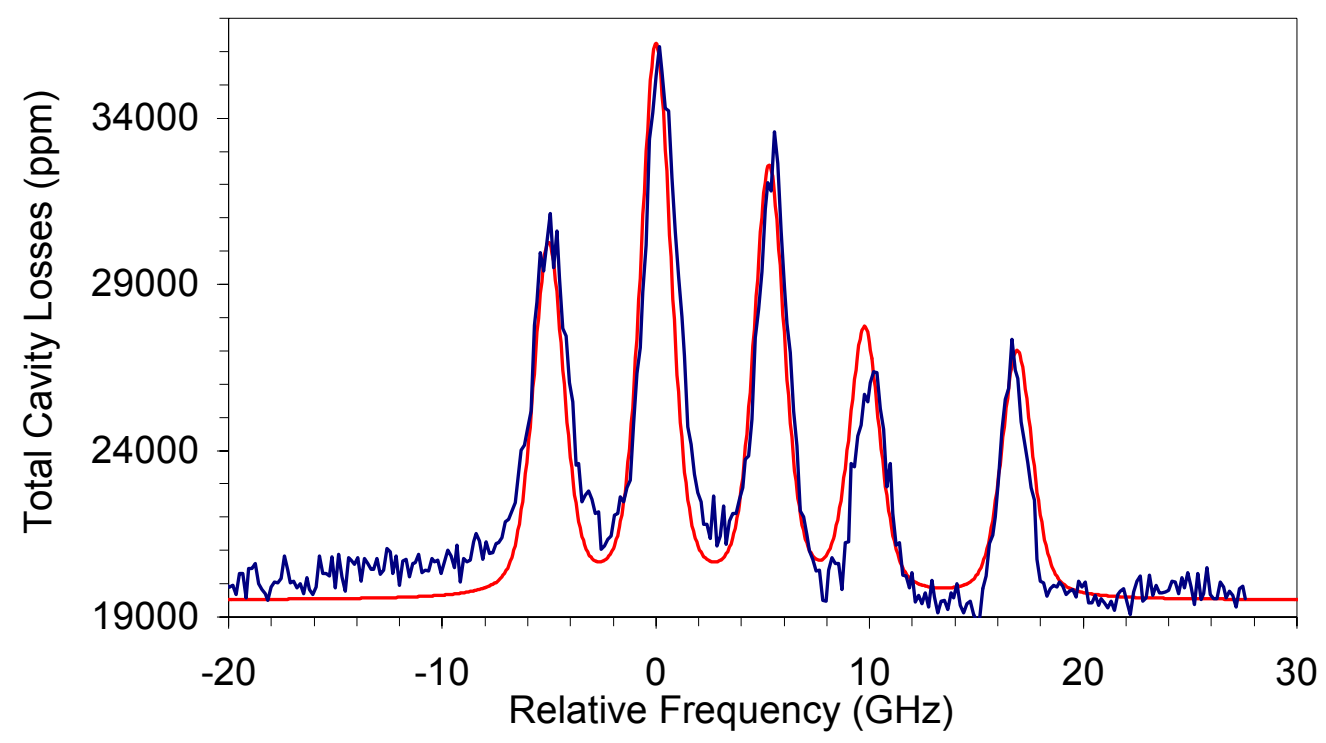

Figure 19: Scan over the atomic mercury isotopic structure with the $38 \mathrm{~cm}$ cavity held at a pressure of 50 torr and a

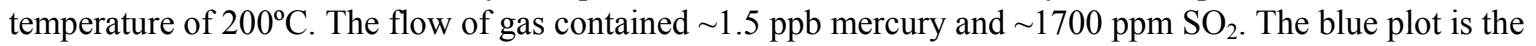
experimental data and the red plot is the theoretically predicted mercury spectrum.

\section{Simulated Flue Gas}

While it is important to be able to determine the baseline losses due to additional absorbing species, the real question remains; can the CRD instrument accurately determine low levels of mercury in the presence of this complex matrix of background gases?

Subsequent to the completion of tests on each individual flue gas component SRD used a gas-mixing manifold to combine a number of the gases and create a "simulated" flue gas mixture to test the CRD instrument. The gases and concentrations included in the mixture were: $1000 \mathrm{ppm} \mathrm{SO}, 285 \mathrm{ppm} \mathrm{CO}, 285$ ppm $\mathrm{CO}_{2}, 70 \mathrm{ppm} \mathrm{NO}, 70 \mathrm{ppm} \mathrm{NO}, 1.8 \mathrm{ppb} \mathrm{Hg}$, and the gas stream was passed through a water bubbler to elevate the concentration of water in the sample stream. This sample stream was sent to the CRD cavity where the pressure was maintained at a constant 100 torr and the temperature at $200^{\circ} \mathrm{C}$. The results are shown in Fig. 20. The figure has two data traces included on it: the first is a plot (blue) of the experimental data obtained from the ring-down time; and the second plot (red) is a theoretical calculation that included the experimental parameters used to take the data with the appropriate concentrations for both mercury and $\mathrm{SO}_{2}$. From the figure it can be seen that there is excellent agreement between the obtained ring-down data and the calculated plot. 


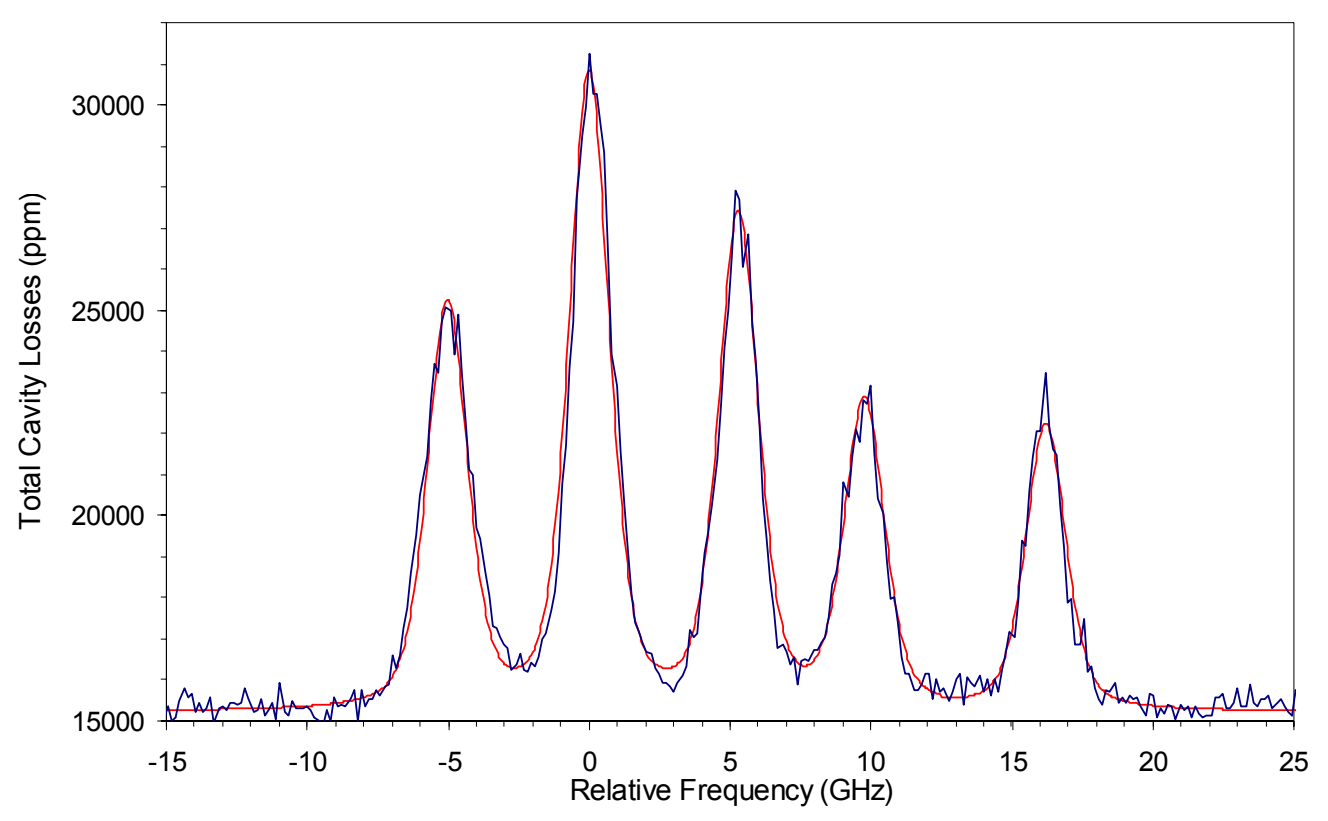

Figure 20: Plot of mercury contained in a "simulated" flue gas. The sample gas stream contained $1.8 \mathrm{ppb}$ mercury, $1000 \mathrm{ppm} \mathrm{SO}, 70 \mathrm{ppm} \mathrm{NO}, 70 \mathrm{ppm} \mathrm{NO} \mathrm{N}_{2}, 285 \mathrm{CO}, 285 \mathrm{CO}_{2}$, and elevated levels of $\mathrm{H}_{2} \mathrm{O}$ from a bubbler. The cavity pressure was maintained at 100 torr and the temperature was held at $200^{\circ} \mathrm{C}$. The blue line is the experimentally determined losses from the ring-down signal and the red line is the calculated value using the experimental parameters including $1.8 \mathrm{ppb}$ mercury and $1000 \mathrm{ppm} \mathrm{SO}_{2}$.

\section{Mercury Speciation}

The aim of this project is to be able to determine the total mercury content in a sample gas stream. However, mercury can be in many different forms such as oxidized $\left(\mathrm{Hg}^{(+2)}\right)$, neutral elemental $\left(\mathrm{Hg}^{(0)}\right)$, or molecular compounds (e.g. $\mathrm{HgCl}_{2}, \mathrm{HgO}$, etc.) containing mercury. One method for determining the total mercury content is to atomize the incoming sample gas stream to ensure all the mercury is in its elemental form, $\mathrm{Hg}^{(0)}$. SRD has chosen to approach this process using the method of pyrolysis. Pyrolysis has a number of advantages over other methods. It is a relatively easy and inexpensive method of atomizing a stream of gas. Other methods such as using a graphite-furnace involve placing the atomization source within the cavity thus significantly decreasing the pathlength available to the species of interest and hence the detection limits attainable with the CRD instrument, given the direct relationship between sensitivity and pathlength. Also, methods involving complicated atomization sources will severely hamper the development of a CRD instrument capable of autonomous operation. 


\section{Sensor Research \& Development Corporation DE-FC26-01FT41221}

SRD has incorporated a Lindberg/Blue M Model TF55030A oven capable of heating the sample gas stream to $1100^{\circ} \mathrm{C}$. This will provide enough energy to atomize molecular species in the gas stream. As well as atomizing all mercury compounds to elemental mercury, the oven can also atomize interfering species such as $\mathrm{SO}_{2}$. While some of the $\mathrm{SO}_{2}$ will recombine, pyrolyzing the entire gas stream may lower any background absorption losses, as none of the atomic species or other reaction products should absorb in the same spectral region of the UV as mercury.

Aside from determining the total mercury concentration there is a great deal of interest in an instrument capable of mercury speciation. In particular the ability to determine the amount of elemental mercury, $\mathrm{Hg}^{(0)}$, from the oxidized form, $\mathrm{Hg}^{(+2)}$, is highly desirable. The binding energy of mercury with other atoms or molecules is generally very weak. This suggests that there may only be a small spectral perturbation to the atomic mercury transition in mercury compounds. It has been reported that a small spectral shift may have been observed for mercuric chloride $\left(\mathrm{HgCl}_{2}\right)$, an oxidized form of mercury, relative to the atomic mercury transition. The resolution of this experiment, done by Spuler and co-workers, ${ }^{3}$ was approximately $3.3 \mathrm{GHz}\left(0.11 \mathrm{~cm}^{-1}\right)$, which was not spectrally narrow enough to resolve the isotopic structure of the mercury transition or definitively establish a spectral shift of the mercuric chloride peak relative to the atomic mercury transition. However, the resolution of the CRD instrument at SRD is approximately $0.18 \mathrm{GHz}\left(0.006 \mathrm{~cm}^{-1}\right)$ in the $\mathrm{UV}$, which is clearly narrow enough to resolve the isotopic structure and may be narrow enough to observe a spectral shift between $\mathrm{HgCl}_{2}$ and atomic mercury. If the shift is present and is great enough it may be possible to determine absolute concentrations of mercury compounds and atomic mercury as well as the total mercury content of a sample.

Initial attempts to atomize the sample gas stream with the pyrolyzing oven set to $800^{\circ} \mathrm{C}$, due to the glass beads used to mix the gases, did not show any definitive results. There was no observable decrease of the background absorption indicating that either the $\mathrm{SO}_{2}$ is just recombining or is not pyrolyzing.

While the initial results were inconclusive there may be a number of reasons for this: first, the glass beads initially used within the quartz tube, to increase heating throughout the sample, limited the operation of the oven to about $800^{\circ} \mathrm{C}$; second, the resident time may not have been long enough to pyrolyze the gas stream; and third, after exiting from the oven there could be recombination or reactions to produce additional species. The addition of quartz beads or chips will allow the temperature to be elevated to the oven limit of $1100^{\circ} \mathrm{C}$. A previous report ${ }^{13}$ of atomizing a gas stream with mercury species indicates a temperature of $1000^{\circ} \mathrm{C}$ should be sufficient for pyrolysis. Adjusting the flow rate can be done to ensue enough resident time in the oven for full pyrolysis. Also, minimizing the distance from the oven to the CRD cavity and heating any tubing between the two can reduce the chances for reaction and/or recombination.

SRD had observed absorptions due to $\mathrm{HgCl}_{2}$ that were in the same region as those belonging to atomic mercury. The next step was to add elemental mercury at the same time to establish if there was any spectral separation between the two species. Figure 21 is a plot of the losses in the cavity with $\sim 1 \mathrm{ppb}$ $\mathrm{HgCl}_{2}$ and $\sim 1.5 \mathrm{ppb} \mathrm{Hg}^{(0)}$. The two gas streams were mixed together just prior to the CRD cavity inlets. The $\mathrm{HgCl}_{2}$ gas stream had heated Teflon tubing from the dynacalibrator permeation oven, containing the $\mathrm{HgCl}_{2}$ permeation tube, to the CRD cavity and was maintained at a temperature of about $200^{\circ} \mathrm{C}$, due to it's tendency to "stick" to the walls of the tubing. 
Prior to the combined experiment each species was introduced individually into the CRD cavity to determine the individual losses due to the $\mathrm{HgCl}_{2}$ and $\mathrm{Hg}^{(0)}$. Included in Fig. 21 is a calculated plot that assumes a total mercury concentration of $\sim 2.5 \mathrm{ppb}$. From the figure it can be seen that the calculated plot agrees very well with the experimental plot for the total concentration. An assumption made in the theoretical plot is that the absorption cross-section for $\mathrm{HgCl}_{2}$ is the same as that for elemental mercury. The agreement between the theoretical predictions and those obtained from the permeation rate are good, which gives this assumption some validation.

It can also be seen that there is no observable spectral separation between the two mercury species. This could be due to a couple of reasons; the first is that any spectral shift of the $\mathrm{HgCl}_{2}$ is too small to be observed with the CRD instrument, or the second possibility is that the $\mathrm{HgCl}_{2}$ has broken down to elemental mercury. The former of the two possibilities seems relatively unlikely while the latter seems to be a much more probable result, although the good agreement between the theoretically predicted spectrum, the permeation rate of the $\mathrm{HgCl}_{2}$ permeation tube, and the experimentally determined spectrum would indicate that all of the $\mathrm{HgCl}_{2}$ would have had to decompose.

While the speciation studies to date have not been able to separate the two forms of mercury the CRD instrument did accurately determine the total mercury concentration. Speciation studies will continue at SRD. This will include efforts to spectrally separate $\mathrm{HgCl}_{2}$ from elemental $\mathrm{Hg}$. Spectral separation would be the most desirable method, as this would lead to unambiguous assignment of the two different mercury species. The selectivity inherent in a laser-based method such as CRD would be able to exploit any spectral separation and individually quantify the two species.

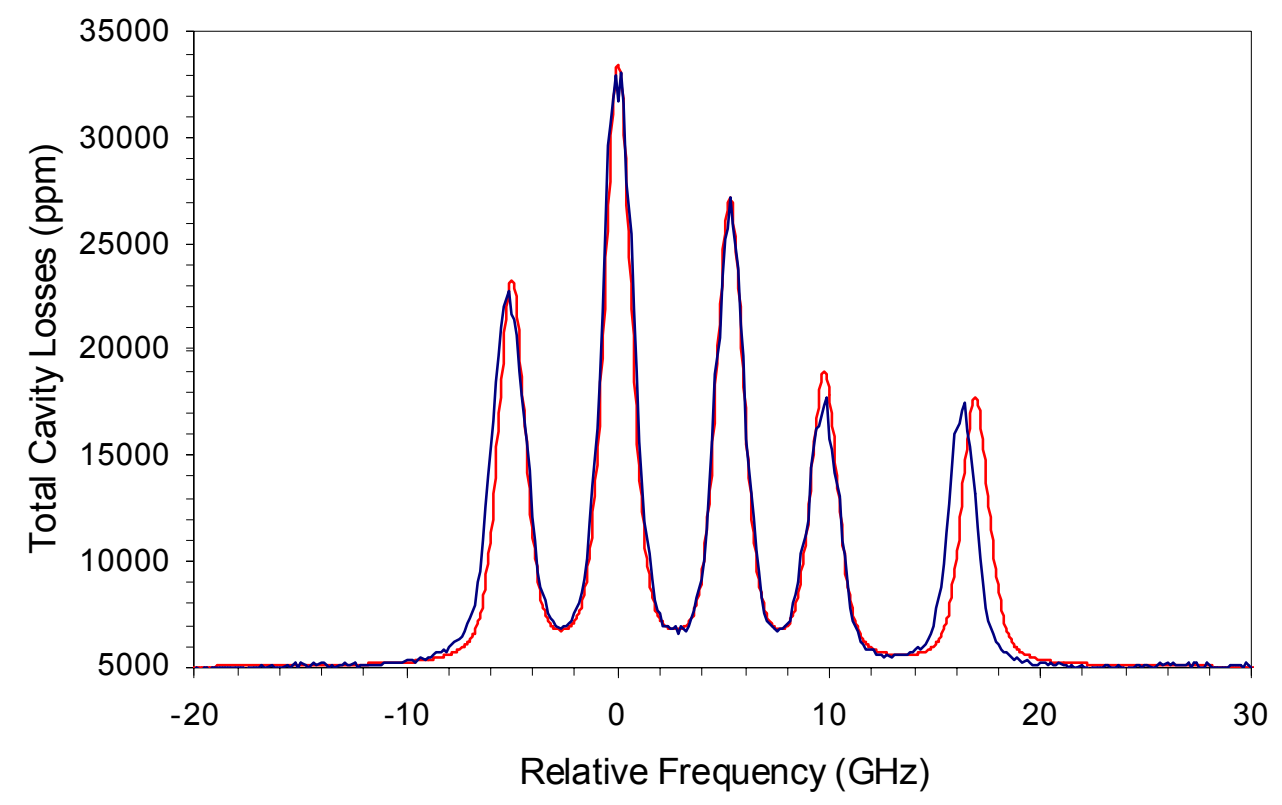

Figure 21: Plot of the total amount of mercury determined from both $\mathrm{HgCl}_{2}$ and elemental mercury. The $38 \mathrm{~cm}$ cell was held at a pressure of 100 torr and temperature of $200^{\circ} \mathrm{C}$. The concentration of the two mercury species was:

$1 \mathrm{ppb} \mathrm{HgCl}_{2}$, and $1.5 \mathrm{ppb} \mathrm{Hg}$. The total concentration used in the calculated plot (red) was $2.5 \mathrm{ppb} \mathrm{Hg}$; the obtained ring-down data is shown in blue. 


\section{Theoretical Modeling}

\section{Cross-Section Calculations}

In order to determine absolute concentrations an accurate model is needed that includes all the experimental parameters: pressure and temperature of the sample gas, molecular absorption crosssections, for the species of interest, naturally occurring isotopic ratios, and resonance frequency positions.

From the obtained ring-down data the absorption coefficient, $\alpha$, is found experimentally by measuring the ring-down decay time, knowing the reflectivity of the mirrors, and the length of the cavity used at a given frequency (see Eq. 1). To find the absolute number of molecules $N$, of the species of interest, and hence the concentration, the absorption coefficient is related to the absorption cross-section by $\alpha=N \sigma$. It is well know that the absorption cross-section of a particular atom or molecule at frequency, $v$, is given by

$$
\sigma\left(v-v_{0}\right)=\frac{g_{k}}{g_{i}} \frac{A \lambda^{2}}{8 \pi n^{2}} \cdot g\left(v-v_{0}\right)
$$

where $v_{0}$ is the resonance frequency, $g_{k}$ and $g_{i}$ are the degeneracy's of the upper and lower energy levels respectively, $A$ is the Einstein coefficient, $\lambda$ is the wavelength, $n$ is the index of refraction, and $g\left(v-v_{0}\right)$ is the molecular line-shape function which is dependent upon pressure and temperature.

In order to simplify the calculations an integrated cross-section is defined as

$$
S=\int_{0}^{\infty} \sigma\left(v-v_{0}\right) d v
$$

which reduces to

$$
S=\frac{g_{k}}{g_{i}} \frac{A \lambda^{2}}{8 \pi n^{2} c}
$$

A factor of the speed of light $\mathrm{c}$ is included in order to express $S$ in its more common units $\left(\mathrm{cm}^{-1} / \mathrm{cm}^{-2}\right)$. Notice $S$ does not contain the line-shape function, as the integrated function is equal to 1 by definition, making it independent of pressure and temperature. This fact makes the integrated cross-section an ideal number to use as a reference value and it is commonly found in papers and reference resources, including the Hitran ${ }^{14}$ database. Unfortunately, for mercury it is not directly given in any of the references found thus far. However, the degeneracy and Einstein coefficient for mercury can be found in the CRC Handbook of Chemistry and Physics ${ }^{6}$ and published journals ${ }^{15}$ :

$$
\begin{aligned}
& g_{k}=3 \\
& g_{i}=1 \\
& A=8.00 \times 10^{6} \mathrm{~s}^{-1} \\
& \lambda=253.652 \mathrm{~nm} \\
& n=1.00
\end{aligned}
$$

Using these values $S=2.05 \times 10^{-14} \mathrm{~cm}^{-1} /\left(\mathrm{cm}^{-2} \cdot\right.$ molecule $)$.

In order to determine the concentration the line-shape function must be evaluated. Being able to evaluate this function at various pressures and temperatures is necessary in order to make the model flexible and to 
determine the best test conditions. For example, decreasing the pressure from atmosphere to 100 torr reduces the molecular linewidth by a factor of 4.5 and allows the five separable isotopic lines to be resolved. It also reduces the absorption strength by a factor of 2.5 .

The linewidth of a particular transition is due to contributions from a number of different mechanisms that can be broken down into two categories: homogeneous and inhomogeneous line broadening. Line broadening due to collisions, or so-called pressure broadening, is an example of the former, while Doppler broadening is an example of the latter. These are the two dominant broadening mechanisms that will be considered here. Homogeneous broadening due to the radiative lifetime, or natural broadening, of the mercury transition is orders of magnitude smaller than the other two effects and will be neglected in this treatment.

At low pressures, Doppler broadening, caused by the distribution in molecular speed, is dominant. The line shape function is given by

$$
g\left(v-v_{0}\right)=\left(\frac{4 \ln 2}{\pi}\right)^{\frac{1}{2}} \frac{1}{\Delta v_{D}} e^{-4 \ln 2\left(\frac{v-v 0}{\Delta v_{D}}\right)^{2}} .
$$

This Gaussian function has a full-width half-maximum (FWHM) Doppler-broadened linewidth given by $\Delta v_{D}=7.1 \times 10^{7} v \sqrt{\frac{T}{M}}$, where $\mathrm{M}$ is the molecular mass and $\mathrm{T}$ is the temperature in Kelvin.

At high pressures, collisional or pressure broadening, dominates. In this case the line shape function is given by

$$
g\left(v-v_{0}\right)=\frac{\Delta v_{\text {coll }}}{2 \pi\left[\left(v-v_{0}\right)^{2}+\left(\frac{\Delta v_{\text {coll }}}{2}\right)^{2}\right]} .
$$

This Lorentzian function has a collisionally broadened linewidth given by $\Delta v_{\text {coll }}=8.996 \cdot P \cdot\left(\frac{273}{T}\right)^{1 / 2}$ $\mathrm{MHz}$. The factor, $8.996 \mathrm{MHz} /$ torr, was determined empirically for mercury in a nitrogen atmosphere by Jacobs and Warrington. ${ }^{16}$

For intermediate pressures a convolution of the two line shapes is necessary since the Doppler broadened molecules will also be collision broadened. This 'Voigt' function is expressed as

$$
g\left(v-v_{0}\right)=\int_{-\infty}^{\infty} g_{\text {coll }}\left(v_{0}^{\prime}-v_{0}\right) g_{D}\left(v-v_{0}^{\prime}\right) d v_{0}^{\prime}
$$


Fortunately, this 'Voigt' function can be approximated ${ }^{17}$ with minimal error. The resulting cross-section approximation is given by the following equations:

$$
\begin{array}{cc}
\sigma_{\mathrm{V}}(v)=\sigma_{\mathrm{V}}\left(v_{0}\right)\left\{(1-\mathrm{x}) e^{-(4 \ln 2) \mathrm{y}^{2}}+\frac{\mathrm{x}}{1+4 \mathrm{y}^{2}}+.016(1-\mathrm{x}) \mathrm{x}\left[e^{-0.4 \mathrm{y}^{2.25}}-\frac{10}{10+\mathrm{y}^{2.25}}\right]\right\} \\
\mathrm{x}=\frac{\Delta v_{\text {coll }}}{\Delta v_{\mathrm{V}}} & \sigma_{\mathrm{V}}\left(v_{0}\right)=\frac{S}{\Delta v_{\mathrm{V}}\left(1.065+0.447 \mathrm{x}+.058 \mathrm{x}^{2}\right)} \\
\mathrm{y}=\frac{\left|v-v_{0}\right|}{\Delta v_{\mathrm{V}}} & \Delta v_{\mathrm{V}}=0.5346 \Delta v_{\text {coll }}+\left(.2166 \Delta{v_{\text {coll }}}^{2}+\Delta{v_{D}}^{2}\right)^{\frac{1}{2}}
\end{array}
$$

Notice at low pressures the value of $\mathrm{x}$ goes to zero and at high pressure it goes to one, making $\sigma_{\mathrm{V}}\left(v_{0}\right)$ approach the values given in the low and high-pressure scenarios above, where no approximation is used.

Using the known relative line positions ${ }^{7}$ and integrated cross-sections for the different isotopes a complete spectral model for mercury was established. With the correct values of integrated cross-section and collision broadened linewidth this same model can be used for any molecule, including the oxygen transition bands found near mercury transitions as well as for $\mathrm{SO}_{2}$.

Shown in Fig. 22 is a plot of the oxygen transition observed near the $253.7 \mathrm{~nm}$ transition of mercury. Along with the assignment of the various lines, to their respective electronic state, is a theoretical plot (shown in blue) using the model described above. It can be seen that the agreement between the theoretical plot and the experimental data is excellent. There is a slight frequency shift of the last spectral line in the plot between the theoretical and experimental plots due to the non-linear behavior of the applied voltage and frequency of the diode seed laser. There has only been a linear correction to the applied voltage conversion to frequency. The agreement achieved provides validation of the theoretical model.

The plot shown in Figs. 23 and 24 are a scan over the $253.7 \mathrm{~nm}$ transition of mercury. The data taken for Fig. 23 was obtained at a cavity pressure of 760 torr and a temperature of $200^{\circ} \mathrm{C}$ with a mercury concentration of about $1.2 \mathrm{ppb}$. The data taken for Fig. 25 was also obtained with a cavity temperature of $200^{\circ} \mathrm{C}$, but the pressure was reduced to 100 torr, and the mercury concentration used was $\sim 75$ pptr. From the two figures it can be seen that the agreement between the CRD data and the data obtained from the theoretical model is excellent.

Figures $22-24$ demonstrate the versatility and accuracy of the model. The model accurately predicts the correct concentrations with changes in both pressure and temperature. As shown in Fig. 22 the model is flexible enough to use with other molecular species as well as mercury. 


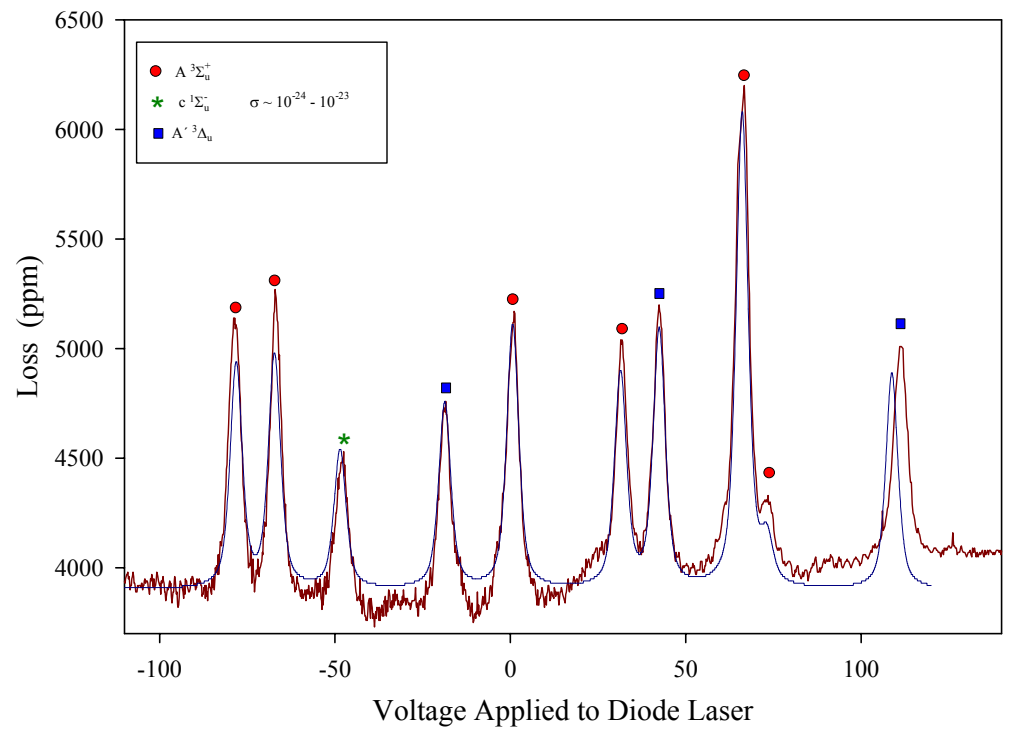

Figure 22: Scan of the oxygen transition originating from the ground $\mathrm{X}^{3} \Sigma_{\mathrm{g}}{ }^{-}$electronic state and terminating on one of 3 excited electronic states labeled on the plot. The absorption cross-section, $\sigma$, for the various transitions is given in $\mathrm{cm}^{2}$. The experimental plot is shown in red while a theoretical plot is shown in blue. The $65 \mathrm{~cm}$ cell contained 400 torr of oxygen and was held at room temperature.

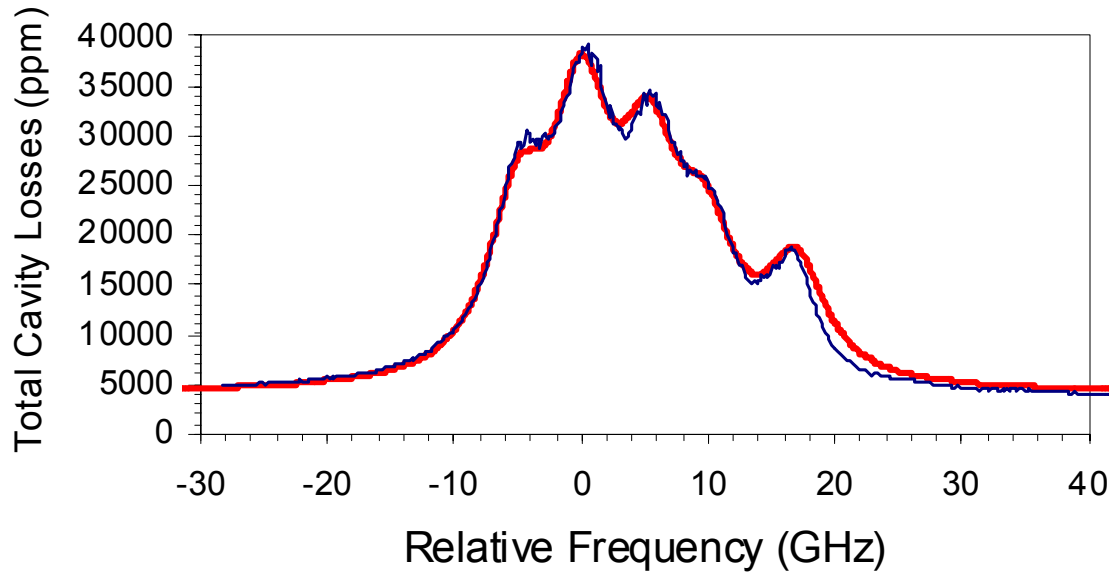

Figure 23: Scan of the $253.7 \mathrm{~nm}$ transition of atomic mercury. The pressure in the $38 \mathrm{~cm}$ CRD cavity was 760 torr, the temperature was held at $200^{\circ} \mathrm{C}$, and the mercury concentration was about $1.2 \mathrm{ppb}$. The full-width halfmaximum of the mercury line is approximately $22.5 \mathrm{GHz}$ due to the isotopic structure of mercury (see text) combined with pressure broadening. 


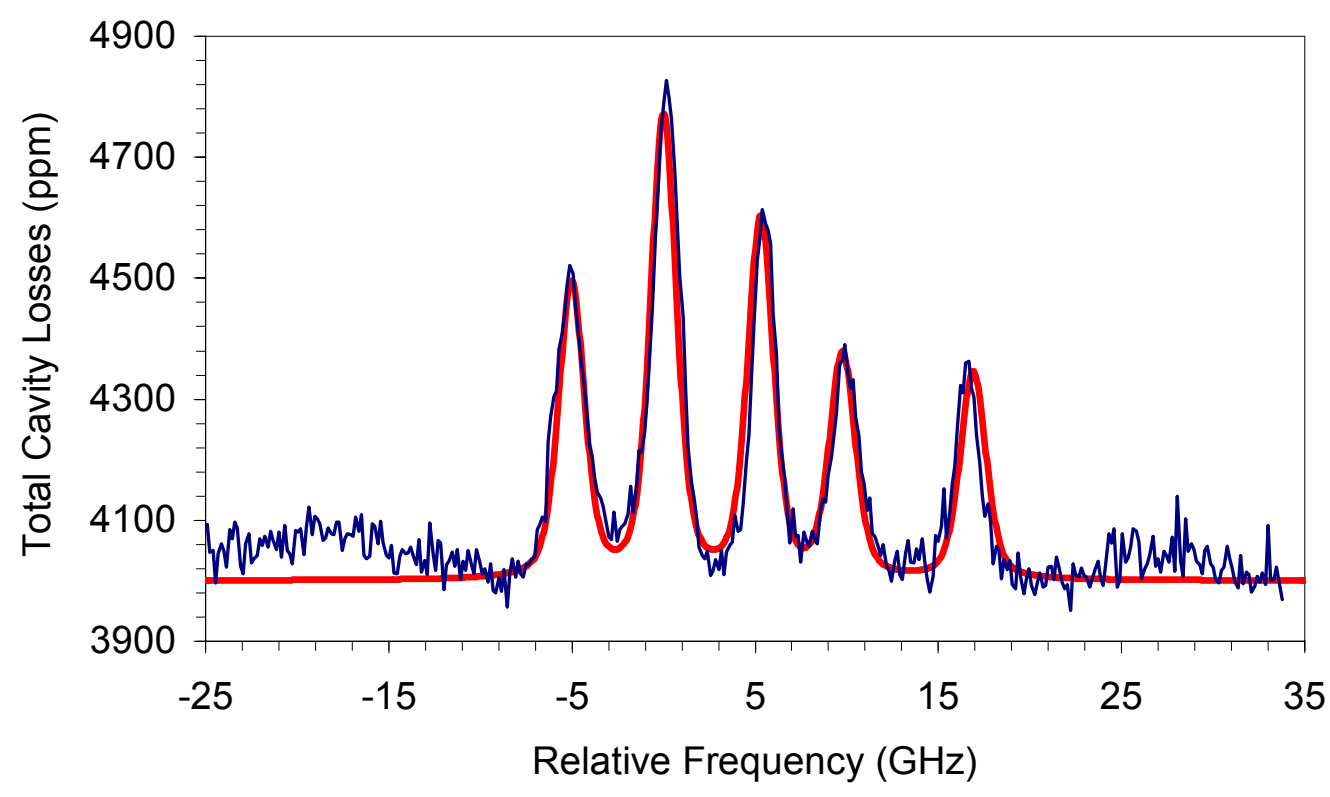

Figure 24: Scan of $\sim 75$ pptr of mercury with the $38 \mathrm{~cm}$ cavity held at a pressure of 100 torr and temperature of $200^{\circ} \mathrm{C}$, showing the resolved isotopic structure of atomic mercury. The plot in blue is the experimental data while the plot in red is the theoretical prediction. Due to the hyperfine splitting some of the peaks contain more than one single line resulting in 5 lines from 6 different isotopomers.

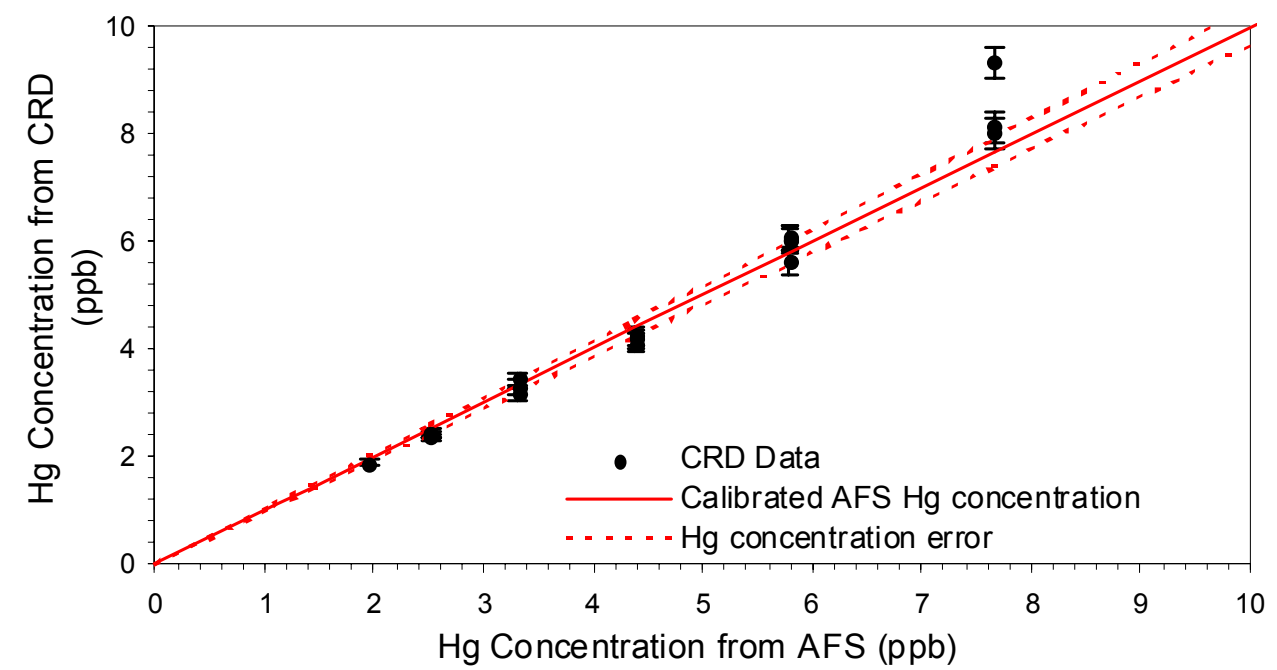

Figure 25: A plot of the mercury concentration determined by the cavity ring-down instrument compared with the mercury concentration determined by the atomic fluorescence spectrometer (AFS).

Further verification of the theoretical model used to calculate mercury concentrations is demonstrated in Fig. 25. This plot is a comparison of the mercury concentration obtained from the permeation oven using the AFS and the concentration obtained from the CRD data using the above-described model. The solid 
red line in the figure is the concentration obtained from the calibrated AFS. The dotted red lines indicating the error associated with the mercury concentration are only due to fluctuations in the temperature of the oven and do not take into account sources of error associated with the AFS measurement itself. The black points on the plot indicate the CRD data and its associated data. From the plot it can be seen that the agreement between the two is very good. Only one CRD data point, out of 14 total, lies outside these reduced error limits.

\section{Determination of the Mercury Peak Position}

One important aspect of the CRD system is the ability of the instrument to automatically determine the total mercury concentration. As mentioned above the CRD technique is self-calibrating, but to accomplish this the laser must change frequency to some off resonance position to measure the baseline losses within the cavity and then return to the mercury peak to measure the absorption losses due to mercury. This allows the determination of the absolute concentration of mercury atoms within the sample stream without running a mercury calibration curve.

While this is one of the great advantages of the cavity ring-down technique the instrument must be able to return to the top of the mercury peak or the concentration returned could be inaccurate. This is slightly more complicated in the case of mercury because of the number of different isotopes. As a result of 6 isotopes, including hyperfine splitting, there are 5 different mercury peaks that can be resolved with the present CRD system. At lower pressures (100 torr) these 5 peaks are completely resolved. Due to pressure broadening the peaks start to overlap, as the pressure is increased to atmospheric pressure (760 torr), and the individual isotopic mercury peaks are more difficult to discern.

A method that can be used to determine whether the laser frequency is located at the correct position, on top of the center of a single isotopic peak, is to take the derivative of the spectrum. As the laser scans across a mercury peak the derivative will go to zero where the slope of the spectrum is zero, i.e. at a minimum or maximum (top of the peak).

To test this method SRD scientists simulated a number of different data sets. These simulations included a random noise level that was set at $10 \%$ of the peak value. This value is higher than that normally observed in the CRD data for mercury concentrations greater than $1 \mathrm{ppb}$. An example of this can be seen in Fig. 26. The parameters used for this simulation were a temperature of $200^{\circ} \mathrm{C}$, a mercury concentration of $1.5 \mathrm{ppb}$ and a pressure of 100 torr. On this, and subsequent plots, the simulated data is displayed as the red plot, the calculated derivative spectrum is the blue line on the plot, and the vertical dotted lines indicate the point at which the derivative is zero and matches a maximum. The other points where the derivative spectrum crosses the zero point correspond to a minimum in the data plot. 


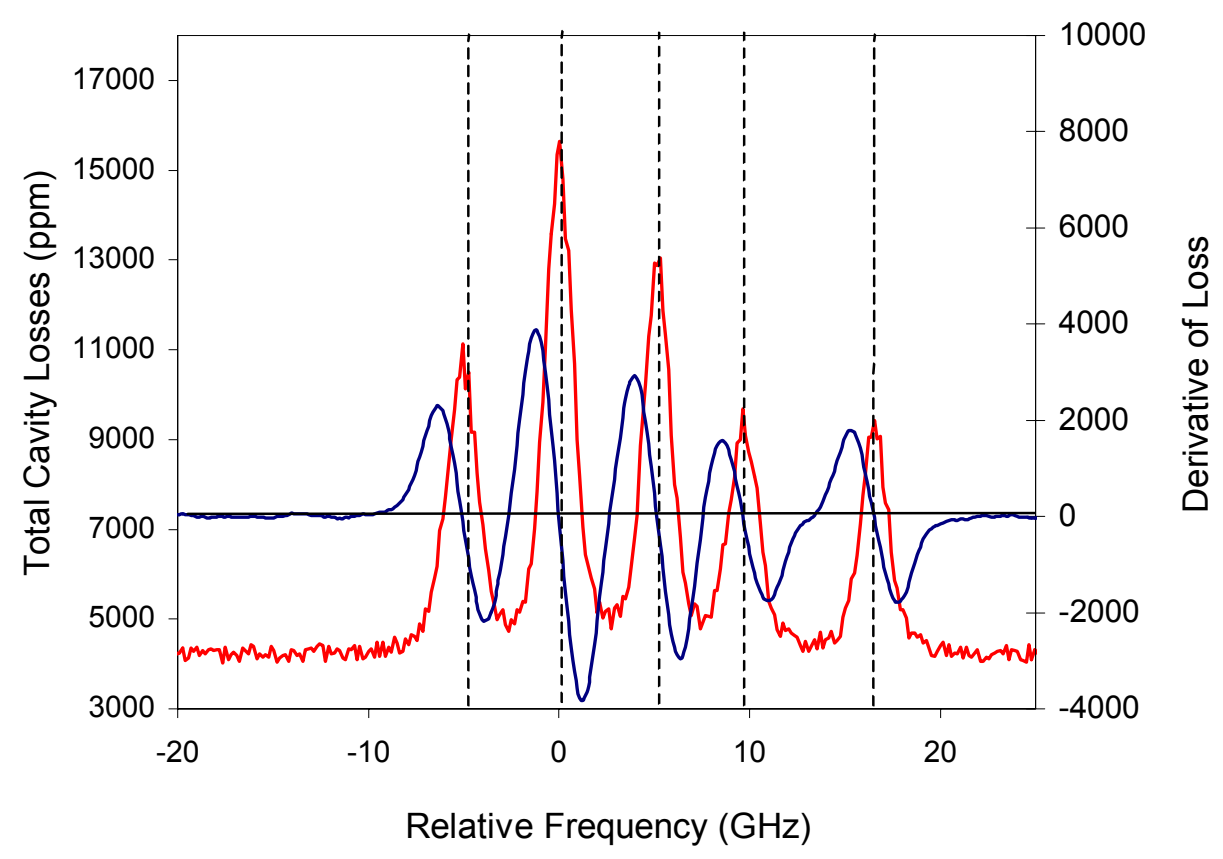

Figure 26: Plot of a computer generated mercury spectrum (red dots) with a simulated noise level of $10 \%$ of the peak height. The solid blue line is a plot of the derivative of the spectrum. The vertical black dotted lines show the position where the derivative crosses the zero point. The parameters used for the simulation were a temperature of $200^{\circ} \mathrm{C}$, mercury concentration of $1.5 \mathrm{ppb}$, and a pressure of 100 torr.

From the plot shown in Fig. 26 it can be seen that the black vertical dotted lines pass directly through the center of the mercury peaks. This indicates that by using the derivative spectrum it is possible to find the position of each of the 5 individual mercury isotopic peaks. The pressure was increased in the simulation to 400 torr. The results are shown in Fig. 27. From this figure it can be seen that indeed the 5 peaks are again correctly identified by the derivative spectrum. However, the amount of the derivative peak that crosses zero for both the first peak, located around $-5 \mathrm{GHz}$, and the fourth peak, located close to $10 \mathrm{GHz}$, is somewhat less than the other three peaks. This can be accounted for by the fact that as the pressure increases and peaks become broader and they become less well defined and the point, at which the slope becomes zero, when it crosses a peak, is harder to determine. 
The pressure used for the simulation was raised to atmospheric pressure (760 torr). The results of the simulated data and derivative plots are shown in Fig. 28. From this plot it can be seen that the derivative plot was only able to correctly identify three of the five peaks. The first and fourth peaks have become small shoulders on the larger peaks and are not well defined enough for the derivative to cross through zero. However, the largest peak was still easily located as was the smallest peak. The largest peak, located at $0 \mathrm{GHz}$, is due to a single isotope and has a large enough natural abundance that the peak is still well defined even at these higher pressures. The smallest peak, located at about $15 \mathrm{GHz}$, is separated enough in frequency that it does not blend into the larger peaks at higher pressures and hence the peak position is still easily determined.

All that is needed to accurately determine the mercury concentration is a single peak. Having both the largest and smallest peaks easily identified is advantageous in that it will increase the dynamic range in which the CRD instrument will function. The largest peak will enable the lowest concentrations to be accurately determined, while the smallest peak can be used for higher concentration levels.

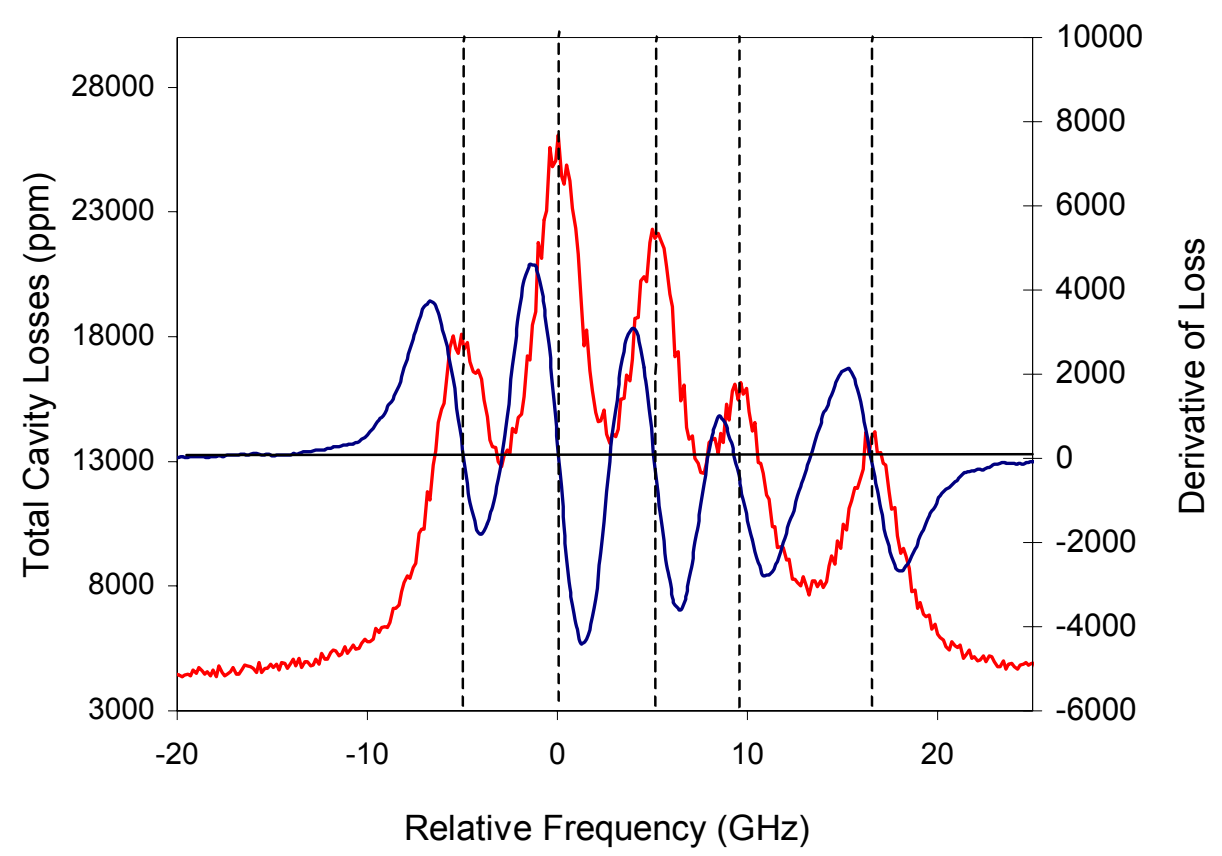

Figure 27: Plot of a computer generated mercury spectrum (red dots) with a simulated noise level of $10 \%$ of the peak height. The solid blue line is a plot of the derivative of the spectrum. The vertical black dotted lines show the position where the derivative crosses the zero point. The parameters used for the simulation were a temperature of $200^{\circ} \mathrm{C}$, mercury concentration of $1.5 \mathrm{ppb}$, and a pressure of 400 torr. 


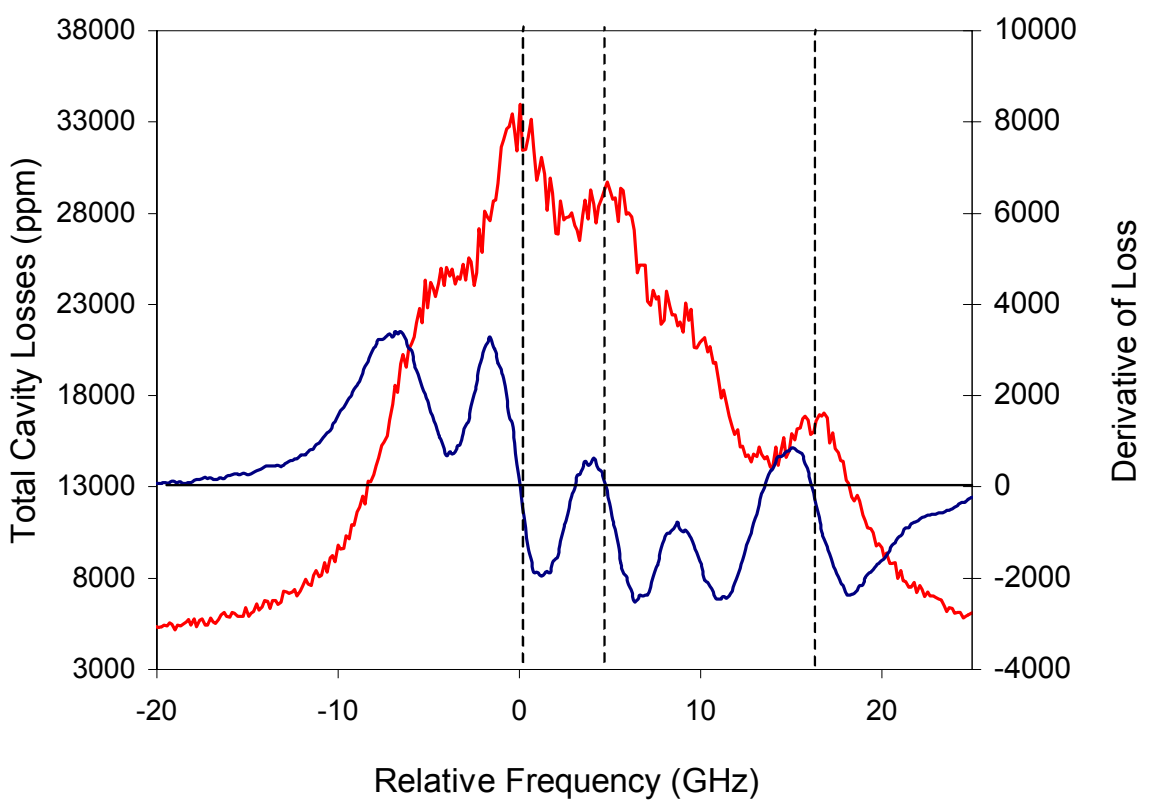

Figure 28: Plot of a computer generated mercury spectrum (red dots) with a simulated noise level of $10 \%$ of the peak height. The solid blue line is a plot of the derivative of the spectrum. The vertical black dotted lines show the position where the derivative crosses the zero point. The parameters used for the simulation were a temperature of $200^{\circ} \mathrm{C}$, mercury concentration of $1.5 \mathrm{ppb}$, and a pressure of 760 torr.

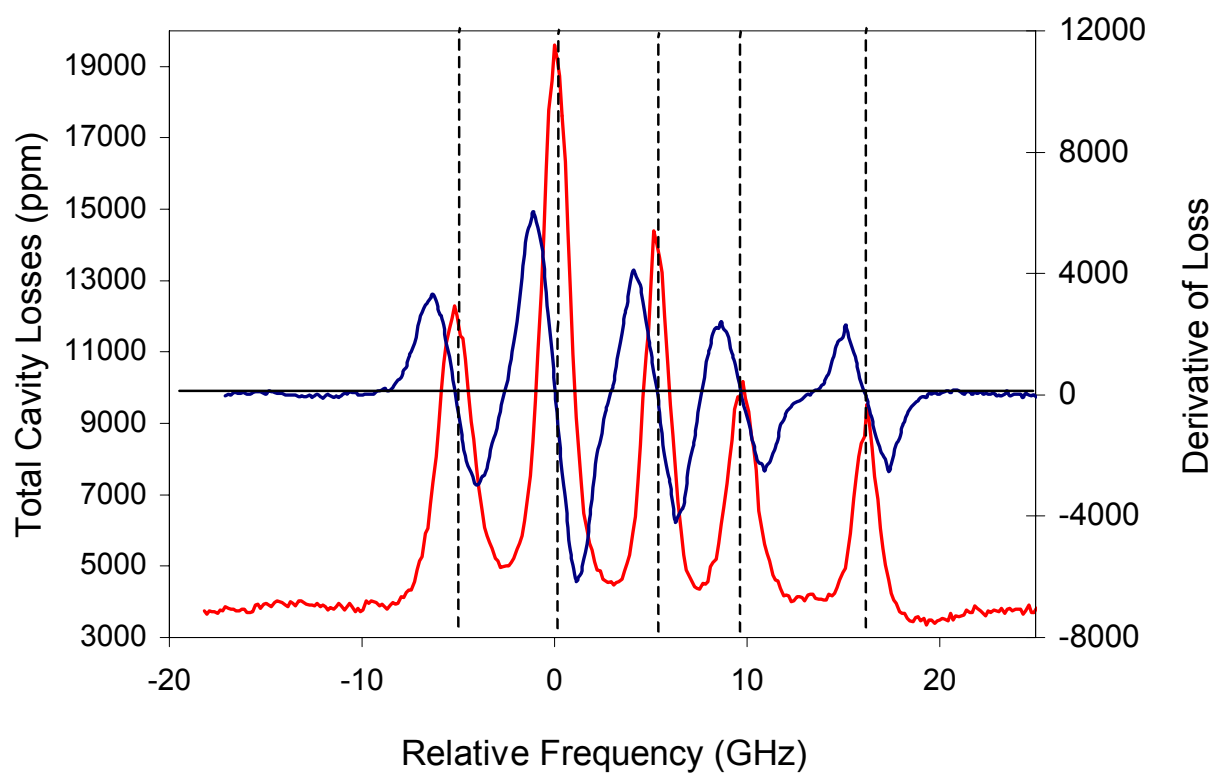

Figure 29: Plot of actual data taken with the CRD apparatus shown by the red plot. The solid blue line is a plot of the derivative of the spectrum and the black dotted vertical lines are drawn through the point where the derivative spectrum crosses zero. The data was taken with a cavity pressure of 100 torr, a temperature of $200^{\circ} \mathrm{C}$, and a mercury concentration of $2.2 \mathrm{ppb}$. 
After obtaining the positive results with the simulated spectra, the derivative method was tested on actual data obtained with the CRD instrument. Shown in Fig. 29 is a plot of CRD data taken at a temperature of $200^{\circ} \mathrm{C}$, a mercury concentration of $2.2 \mathrm{ppb}$ and a cavity pressure of 100 torr. Here again the derivative spectrum is plotted with a solid blue line. It can be seen from the plot that the vertical black dotted lines, indicating where the derivative crosses the zero line, are located in the center of each of the 5 different isotopic mercury peaks, as was observed for the simulated spectrum.

Shown is Fig. 30 is a similar plot where the mercury concentration is $1.7 \mathrm{ppb}$ and the cavity pressure has been raised to 450 torr. From the derivative spectrum and the vertical lines it can be seen that again each of the 5 peaks has been correctly identified. Similar to the simulation for 400 torr, the first and fourth peaks are less well defined and as such have a shallower zero crossing.

Figure 31 is a plot of experimental data taken with a mercury concentration of $1.7 \mathrm{ppb}$, a temperature of $200^{\circ} \mathrm{C}$ and a pressure of 600 torr. From the plot it can be seen that even at a pressure close to atmospheric the derivative method was able to correctly identify 4 out of 5 mercury isotopic peaks.

From the simulated and experimental plots depicting the derivative method, even in the worst case scenario, the method could still identify 3 mercury isotopic peaks, while for the majority of cases it could correctly identify the peak position of all 5 peaks. This is quite promising as a method for the CRD instrument to automatically, and correctly, identify the different isotopic peak positions of mercury.

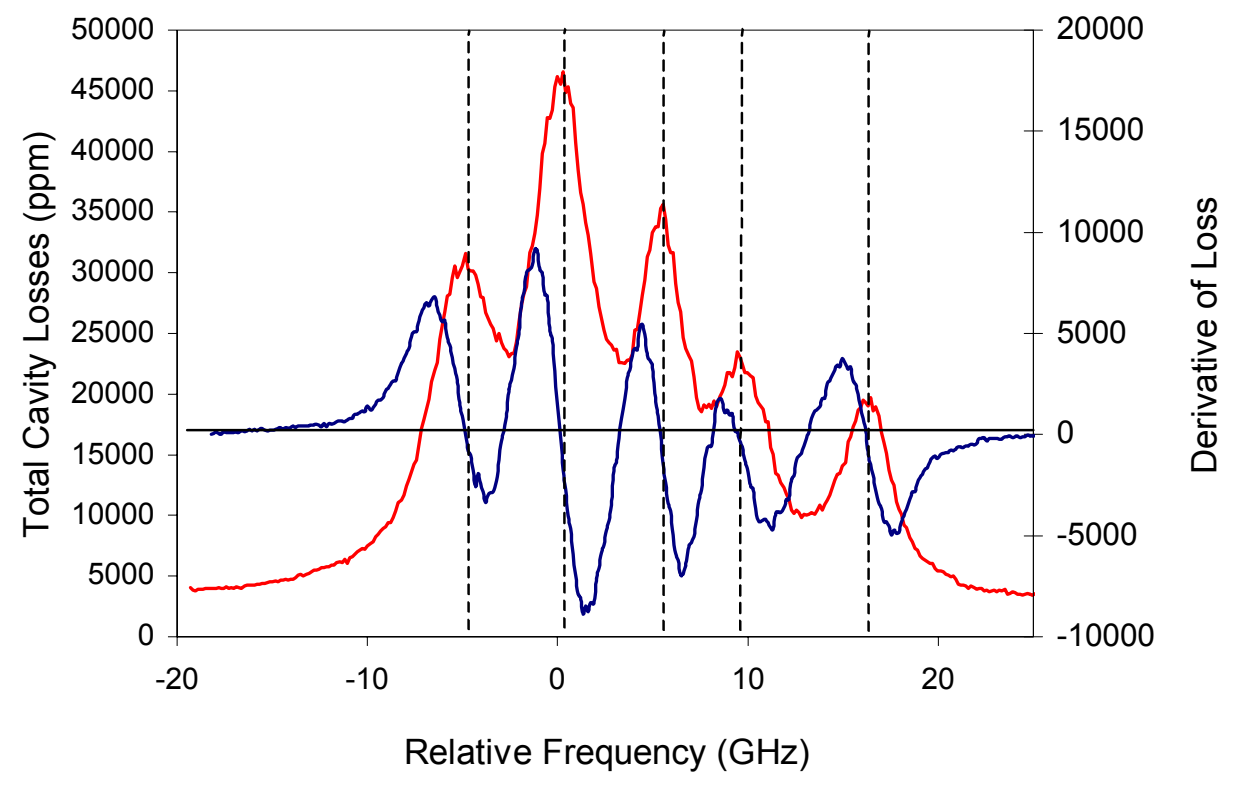

Figure 30: Plot of actual data taken with the CRD apparatus shown in red dots. The solid blue line is a plot of the derivative of the spectrum and the black dotted vertical lines are drawn through the point where the derivative spectrum crosses zero. The temperature of the cavity was held at $200^{\circ} \mathrm{C}$, a pressure of 450 torr, and a mercury concentration of $1.7 \mathrm{ppb}$. 


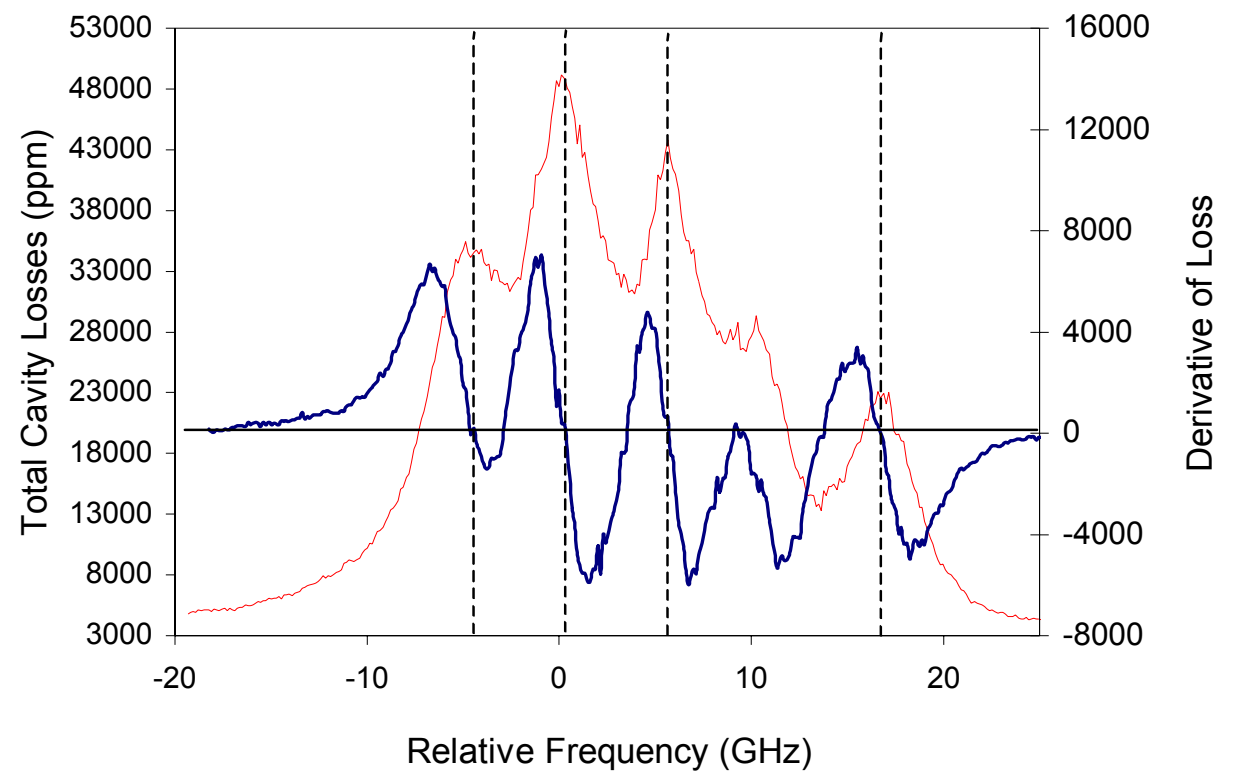

Figure 31: Plot of actual data taken with the CRD apparatus shown in red dots. The solid blue line is a plot of the derivative of the spectrum and the black dotted vertical lines are drawn through the point where the derivative spectrum crosses zero. The mercury concentration used was $1.7 \mathrm{ppb}$, the temperature of the cavity was held at $200^{\circ} \mathrm{C}$, and the pressure was maintained at 600 torr.

\section{Sampling System}

\section{Commercial Off The Shelf Components}

Initial investigation of the sampling system began with the evaluation of commercially available products. A variety of these products are delineated below. Included with each product is a summary of its function as well as cost estimates, where they were available.

\section{$\underline{\text { Apogee Scientific, Inc. }}$}

\section{Quick Silver Inertial Separation (QSIS)}

$\$ 9,250$. to $\$ 10,250$.

The QSIS probe system is used to extract and filter flue gas samples from coal-fired power generating plants for the purpose of determining total vapor-phase mercury concentrations. The probe is designed to deliver these samples with minimal change to vapor-phase mercury speciation. The QSIS system is mounted external to the flue gas exhaust stack and is the interface between this stack and a mercury sample processing system. Typical installation is for CEM applications. 
QSIS is primarily a low maintenance filter system that is both flow and temperature controlled. Principal components are an inertial separation assembly and a porous filter element. Filtering is for particles $2 \mu \mathrm{m}$ and above $(0.5 \mu \mathrm{m}$ available with a concomitant increase in filter maintenance). Probe system flow rate, typically set around 10-12 acfm is managed by a closed loop flow control system. This includes a magnahelic gauge to measure pressure and is linked to a blower system that powers a pneumatic pump on the return side of the sample probe. Sample flow rate, the amount siphoned off for Hg measurement, is typically in the $\leq 4 \mathrm{slpm}$ range. Flue gas not drawn off for analysis is returned to the exhaust stack. Temperature control is done in 2 zones, with the pre-filter zone set to match the flue gas temperature $\left(\cong 150^{\circ} \mathrm{C}\right)$ and the post-filter section set to a slightly higher temperature $\left(\cong 180-200^{\circ} \mathrm{C}\right)$. There are 3 sample ports with $1 / 4$ " Swagelok connections. One of the ports is used for sampling total $\mathrm{Hg}$, one for elemental $\mathrm{Hg}$, and the third for a thermocouple to monitor filter temperature. For non-isokinetic sampling, the nozzle internal to the exhaust stack is normally just a $3 / 4 "$ O.D. stainless steel (SS) tube cut to the appropriate length and connected to the QSIS by a Swagelok to NPT fitting. The QSIS system is not normally used in an isokinetic configuration however it has been successfully adapted to this technique.

Apogee QSIS systems have been installed and used at the DOE/NETL facility in Pittsburgh, Pa., at the UNDEERC/DOE facility in North Dakota, and Western Kentucky University as well as with a variety of research and CEM service providers.

\section{$\underline{\text { Apex Instruments }}$}

Apex manufactures a variety of instruments designed to comply with EPA reference methods for isokinetic and gaseous sampling of flue gas emissions. They also sell individual components to clients fabricating their own sampling systems.

\section{SY5-CIS Method 5 Sampling Train $\mathbf{\$ 1 3 , 0 0 0 .}$}

The Method 5 Sampling Train is a complete yet portable isokinetic sampling system designed to meet EPA Method 5 requirements. It features a heated 6' sampling probe (can be ordered at any suitable length), which includes the glass lined probe itself, a Pitot type gas sampler for the manometer, probe heater and thermocouple, and can be ordered with a variety of accompanying test apparatus such as gaseous sample probes etc. This is followed by a heated sampling box, which supports a filter assembly and the appropriate set of impinger glassware. Finally, there is the model 572 source sampler console which houses the temperature/thermocouple controls and power supplies, a dual manometer, sample pump, gas meter, and umbilical cable connections. The heated umbilical cord (60' plus a 15' extension), the packing/transport cases, monorail assembly, as well as a variety of support tooling is also included.

PA-4G-O2 Method 5 Probe Assembly with oversheath \$591.

Glass lined 4' probe assembly for isokinetic sampling without any attachments or support equipment.

SK-CM5 Compact Method 5 sampling system $\quad \$ \$ 4270$.

Includes a 6' standard heated Method 5 probe, filter assembly, unheated Teflon lined sample line, set of nozzles, and a set of stainless steel impingers along with a case.

\section{RS-PS2-SYS PS2 RATA Sampler \$2995.}

This is a probe designed to do gaseous (not isokinetic) sampling of flue gas emissions in CEM (Continuous Emission Monitoring) mode. It consists of a heated probe with 3 individual sampling tubes, a filter, a small console to control temperature and a magnahelic used to ensure equal flow rates from all 3 sampling tubes. There is also a $100^{\prime}$ sample line. The system as packaged does not include pumps or impingers. 


\section{PC4-4S CEM Probe Assembly}

$\$ 245$.

This is a permanently mounted probe for use as a gaseous CEM. It includes a 4' CEM probe assembly with a sheath, heater, thermocouple, and $1 / 4$ " SS liner, mounted on a universal pipe flange reducer. The liners come in various diameters and lengths.

GNFA-2 Glass Filter Assembly + glass fiber filter $\quad \$ 195$. $+\$ 46$

The glass filter housing is designed to go in-line for particulate filtering.

SFA-47 In-stack SS Filter Assembly $\$ \mathbf{\$ 2 9 5}$

This filter assembly is designed to be located immediately after the sample nozzle within the exhaust stack. It does not include a nozzle.

NS-SET Isokinetic Nozzle Set \$427.

A set of 7 SS nozzles (for different sample rates) used as the point source for collection of isokinetic flue gas samples. Can also be purchased in glass, Quartz and Inconel.

\section{HSRL Self Regulating Heated Sample Line \$25-\$28 / ft.}

A self-regulating sample line that maintains a temperature of $150^{\circ} \mathrm{C}$, has a Teflon tube core, and is insulated with a braided protective sleeve. This can be ordered with more than one tube core and in a variety of tube sizes. Lengths from $10^{\prime}$ to 100 ' are available.

ICS-xxx CEM Temperature Controllers $\$ 3398-595$

These are one and two station temperature controllers. They can be used for thermocouple-controlled equipment or for self-regulating heated sample lines.

\section{Clean Air Express}

Clean Air Express sells and rents equipment used for CEMs and flue gas analysis. They also offer consulting and training services.

0723-100 Heated CEM Teflon Tubing \$2878.

A heated umbilical line for CEM applications, 100' long. It contains a 3/8" Teflon line, a 1/4" Teflon line, 2 type $\mathrm{K}$ thermocouple lines. This heated tubing comes in a variety of sizes and lengths with the shorter lengths costing more per foot.

\section{$\underline{\text { O’Brien Corp. }}$}

Manufacturers of tubing bundles for a variety of applications such as stack gas analyzers, gas chromatography, water analyzers, and other scientific instrumentation.

SU-S2-T-S120/100/K15 Heated Teflon Tubing $\$ \mathbf{\$ 1 8 5 0 .}$

$100^{\prime} \times 1 / 4 "$ heated/insulated Teflon tubing with thermocouple.

SU-S2-T-S120/100/K15 Heated Teflon Tubing $\$ \$ 960$.

40 ' x 1/4" heated/insulated Teflon tubing with thermocouple.

HC10-K-120 Digital Temperature Controller

$\$ 533$.

Temperature controller and power supply for O'Brien heated Teflon tubing. 


\section{Clayborn}

Manufacturer of electrical resistance heating tape and heated tubing assemblies.

\section{$\underline{\text { Dekoron-Unitherm }}$}

Manufacturer of insulated tubing products and controllers. They will make custom lengths, voltage, etc.

210-4-10ft. K-115-T Series 200 Electrically Heated Hose

$\$ 742$.

Outdoor heated 1/4" Teflon cored hose, 10' long with thermocouple.

210-4-30ft. $\quad$ K-115-T Series 200 Electrically Heated Hose \$1216.

Outdoor heated $1 / 4$ " Teflon cored hose, 30' long with thermocouple.

210-4-60ft. K-115-T Series 200 Electrically Heated Hose $\quad \$ 1847$.

Outdoor heated 1/4" Teflon cored hose, $60^{\prime}$ long with thermocouple.

Series 80 Controller

$\$ 387.60$

Thermocouple regulated power source for heated hose.

\section{Diebolt Co.}

Designer and manufacturer of specialty hose assemblies. All hoses quoted have a single $1 / 4$ " Teflon tube core, J type thermocouple, insulation, abrasion resistant jacket, and have Swagelok end terminals. These are listed at $240 \mathrm{VAC}$ but can be fabricated for $115 \mathrm{VAC}$. The number preceding the $\mathrm{J}$ is the length of the heated hose.

$\begin{array}{llc}\text { DTM-5-6'-J-240-Swagelok } & \text { Heated Hose } & \$ 237 . \\ \text { DTM-5-10'-J-240-Swagelok } & \text { Heated Hose } & \$ 347 . \\ \text { DTM-5-40'-J-240-Swagelok } & \text { Heated Hose } & \$ 697 . \\ \text { DTM-5-60'-J-240-Swagelok } & \text { Heated Hose } & \$ 997 . \\ \text { DTM-5-100'-J-240-Swagelok } & \text { Heated Hose } & \$ 1497 .\end{array}$

\section{Teknocraft Inc.}

This company designs and manufactures fluid control products, primarily valves.

Mini Proportional Control Valve

$\$ 392.70$ (12vdc w/.125 orifice)

DC driven proportional control valve, 1/8" NPT inlet port, 6, 12, or $24 \mathrm{vdc}$, with orifice sizes .032, .062, $.093, .125 "$. 


\section{Sensor Research \& Development Corporation}

Signal Amplifier

$\$ 200$.

Used to interface with computer, potentiometer, or PLC control system

$\underline{\text { Air Dimensions, Inc. }}$

Manufacturer of specialty diaphragm type non-corrosive pumps systems used for contamination free pumping of gases and liquids.

R221-BT-AA1-M Heated Head, Teflon Coated Aluminum Diaphragm Pump \$1190.

The diaphragm pump and motor have a rated pumping speed of $27 \mathrm{lpm}$. It is heated to $200^{\circ} \mathrm{C}$, operates with $115 \mathrm{vac}$, and has $1 / 4^{\prime \prime}$ female NPT fittings.

From the list given above it can be seen that there are a number of possible options for the sampling system, which will integrate with the ring-down cavity. SRD has chosen to use the QSIS probe from Apogee Scientific to extract a sample gas stream from the flue gas stack, when particulate filtering is needed. This probe has been used successfully at the DOE/NETL facility in Pittsburgh as well as a number of other facilities. The QSIS sample probe will only be needed for samples taken before the baghouse prior to particulates being removed from the flue gas stream. Sampling after the baghouse can be accomplished with simple stainless steel tubing inserted into the flue gas duct.

SRD engineers designed a sampling system that is suited to the needs of the CRD instrument. This includes components such as a pumping system to maintain the CRD cavity at any pressure from about 50 torr up to atmospheric pressure (760 torr) or slightly above. Other unique components include a pyrolysis oven and a calibration air source. Details of the system are given below.

Shown in Fig. 32 is a diagram of the sampling system with the major components listed on the figure. The flue gas is extracted from the stack using the Quick Silver Inertial Separation (QSIS) probe system from Apogee Scientific. The probe is designed to provide particulate matter free samples with minimal change to vapor-phase mercury speciation. It is important the sample be free of particulate matter in order to avoid excess scattering losses during the ring-down measurements. The sample for the CRD system is pulled from the QSIS system by a vacuum pump that is located after the CRD cavity. The flow rate through the CRD system is typically between 200 and $1000 \mathrm{ccm}$. However, precisely known flow rates are not necessary since the CRD technique directly measures the absolute concentration of mercury atoms instead of deriving it from a measured quantity (i.e. fluorescence or absorbance) and then using calibration curves. Once analyzed the sample flue gas is returned to the flue stack.

To ensure no mercury is removed from the flue gas sample, all gas delivery lines are heat-traced to prevent mercury adsorption to surfaces. The majority of these lines are heated Teflon tubing, which is heated to over $150^{\circ} \mathrm{C}$. The pump is also Teflon coated and the CRD cell is heated with heating tape.

In order to determine the total amount of vapor phase mercury the flue gas sample is heated in a pyrolyzing oven to $1000^{\circ} \mathrm{C}$ to atomize any mercury species, such as mercury oxide and mercuric chloride. This pyrolysis technique will convert all forms of mercury to elemental mercury. 


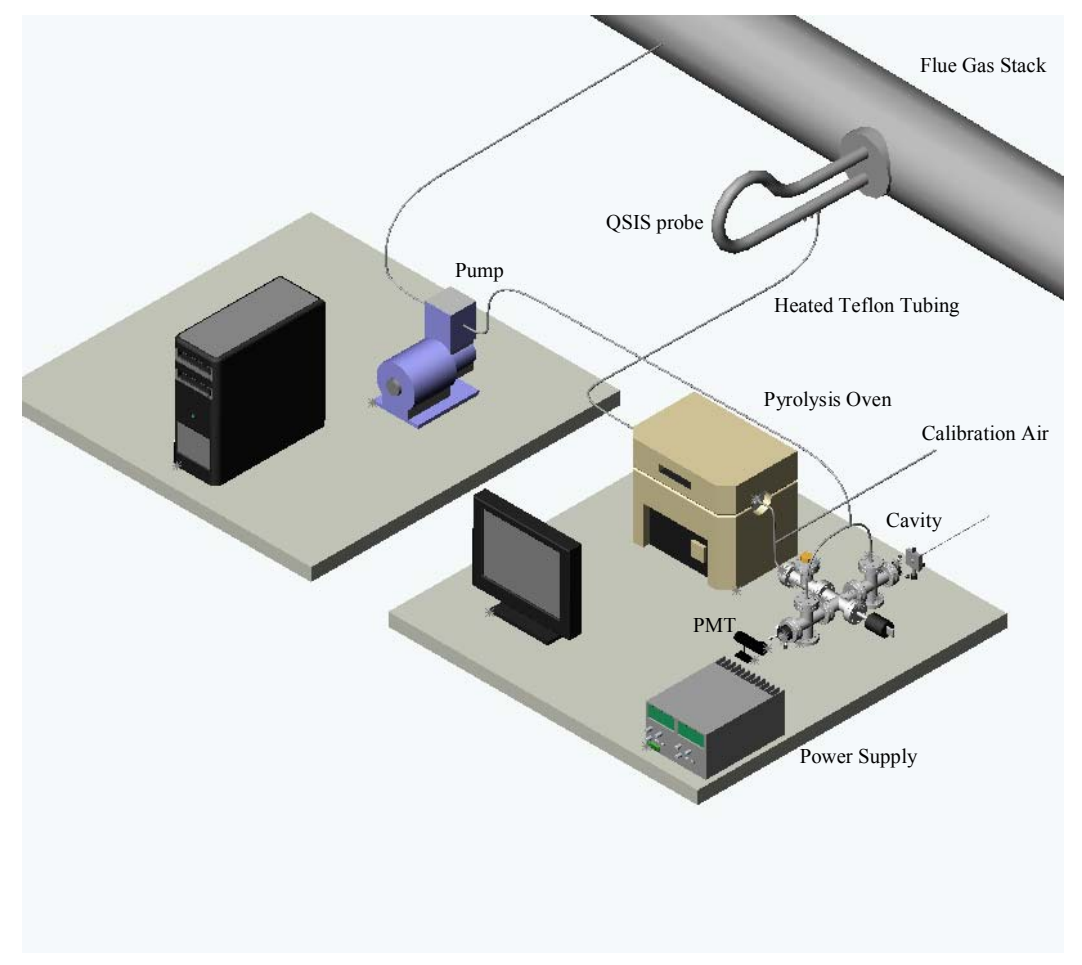

Figure 32: Diagram of the sampling system that will be used to extract a sample gas stream from the flue gas stack, carry it through the pyrolysis oven where all the mercury species are converted to elemental mercury, move it through the cavity where the mercury concentration is determine, and return the gas stream to the stack. The major components are listed on the figure.

The CRD technique is self-calibrating by tuning the laser frequency on and off the molecular absorption line. Mercury has several distinct absorption peaks any of which can be monitored to report an accurate mercury concentration. However, sulfur dioxide $\left(\mathrm{SO}_{2}\right)$ has a broad structureless absorption in the same wavelength region as the mercury transition (see section on Interferent Gas Testing). To separate this sulfur dioxide signal from the mirror baseline, and to ensure the high reflectivity of the ring-down mirrors has not been sufficiently degraded by contaminates, a three-way gas flow-valve is used to periodically switch from flue gas to calibration air or nitrogen to monitor the baseline losses of the cavity with no absorbing species present within the ring-down cell.

A pressure gauge and thermocouple monitor the pressure and temperature of the gas inside the cell, as both are important parameters in correctly determining the concentration of mercury. In order to correct for fluctuations in these variables this information is continually relayed to the computer and saved with the corresponding ring-down data. Throttling valves, before and after the CRD cell, are used to actively control the pressure and flow rate in the cavity. 


\section{Sensor Research \& Development Corporation DE-FC26-01FT41221}

The Alexandrite laser system, used to produce the desired $254 \mathrm{~nm}$ radiation, is fiber optically coupled into the cell with a fiber positioning system and coupling optics. The beam path is entirely enclosed to minimize the hazards of stray laser light. After passing through the cell many times the laser decay signal or ring-down signal is observed with a PMT detector. The computer collects the ring-down data, where it is used in conjunction with the pressure and temperature data to determine accurate mercury concentrations. Since the baseline losses of the instrument are monitored the concentration of $\mathrm{SO}_{2}$ in the cell is also reported, as it is the only other species to absorb in the same region as the mercury transition.

\section{Fiber Optic Coupling}

Presently the laser source that is used to produce the ultra-violet (UV) radiation, required for the mercury transition that is injected into the ring-down cavity, is somewhat large and cumbersome. Remote analysis of flue gas emissions from coal-fired power plants could therefore be a very challenging proposition. A method of alleviating this problem is to isolate the laser source at some point and couple the output radiation into an optical fiber, which can then easily carry the UV beam to the CRD cavity. However, since the radiation is relatively deep into the UV range $(\sim 253.7 \mathrm{~nm})$, transmission of the beam through an optical fiber is not as readily accomplished as transmission in the visible or infrared regions. The material the fiber is made of must obviously transmit the desired radiation. There is also the problem of solarization, the process by which the transmission properties of the optical fiber degrade with time due to the harsh UV radiation. SRD has obtained a solarization-resistant optical fiber from Polymicro Technologies, LLC. This 3-meter fiber has been tested and found to transmit the $254 \mathrm{~nm}$ radiation with very little observable losses.

Once the light was coupled into the fiber a couple of lenses were needed to take the output beam, modematch it to the ring-down cavity, and inject the light into the cavity. SRD scientists setup the appropriate optics and were able to obtain the necessary ring-down time needed to acquire the signal to detect the concentration of mercury.

Upon completion of the steps needed to take the UV laser beam from the laser output, couple it into and transmit it through the optical fiber, and then inject the light into the ring-down cavity, the next step was to test the system by measuring the mercury transition. Shown in Fig. 33 is a plot of the mercury ringdown signal obtained at room temperature, with a cell pressure of 870 torr, and a mercury concentration of about $1.8 \mathrm{ppb}$. The blue line shown in the plot is the experimentally obtained data while the red line is the theoretical plot, using the model developed by SRD, for the given temperature, pressure and concentration of mercury. As can be seen in the plot the agreement between the theoretical plot and the experimental data is excellent. The concentration of mercury has been verified with an atomic fluorescence spectrometer.

The pressure in the cavity was reduced to resolve the isotopic structure of the mercury transition to ensure proper modeling of each of the isotopic peaks as well as verify accurate operation over a wide range of cavity pressures. The plot shown in Fig. 34 depicts the results of scanning the diode seed laser over the mercury transition at a cavity pressure of 90 torr, with the cell maintained at room temperature, and a mercury concentration of $1.85 \mathrm{ppb}$. As shown in both Figs. 33 and 34, the blue line represents the experimentally determined data while the red plot is the theoretical plot for the given experimental parameters. Here again we see excellent agreement between the experimental data and the theoretically produced plot. 


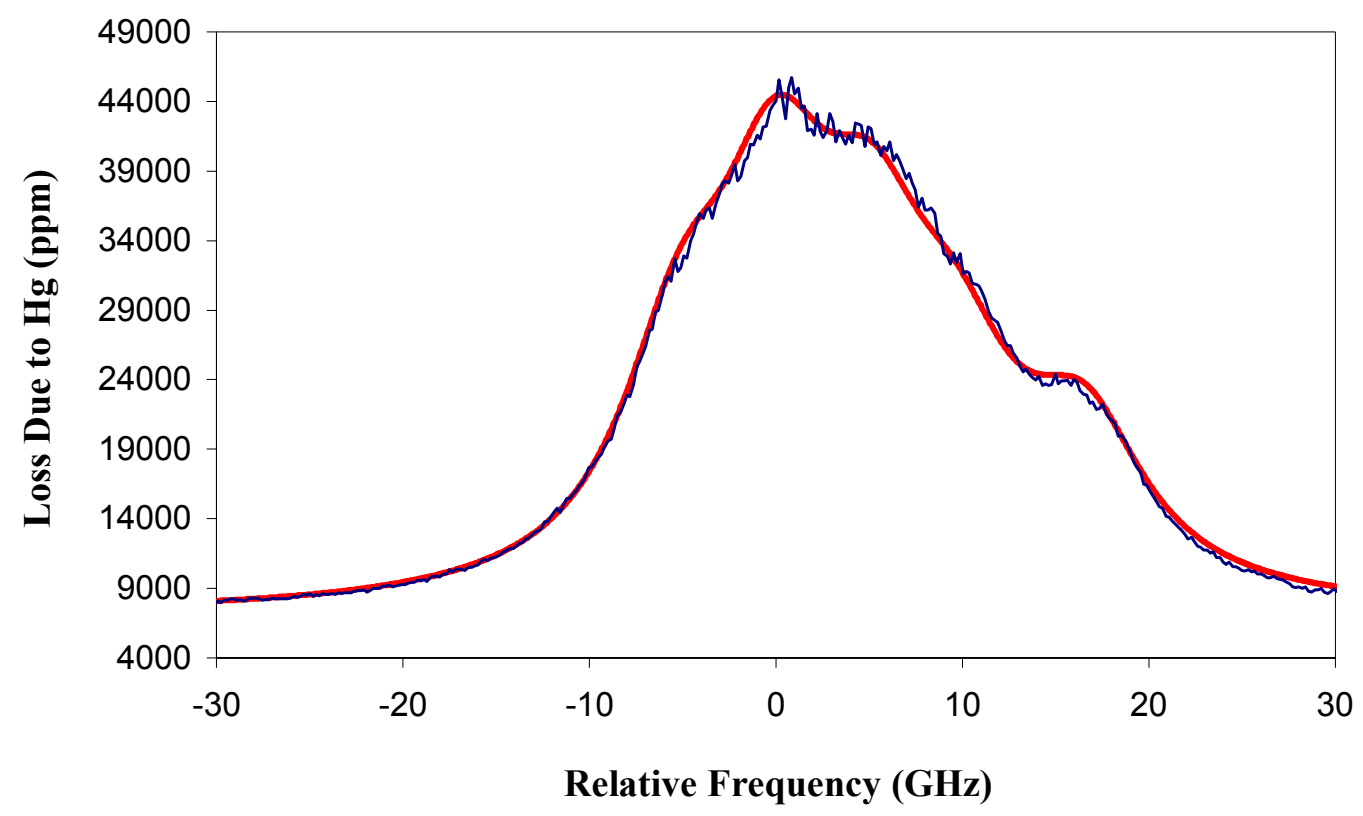

Figure 33: Plot of the relative frequency of the diode seed laser versus the losses in the cavity. The data was obtained with the cell maintained at room temperature, 870 torr pressure, and about $1.85 \mathrm{ppb}$ of mercury. The blue line is the experimentally obtained data and the red line is a theoretical plot fit to the parameters used in the experiment.

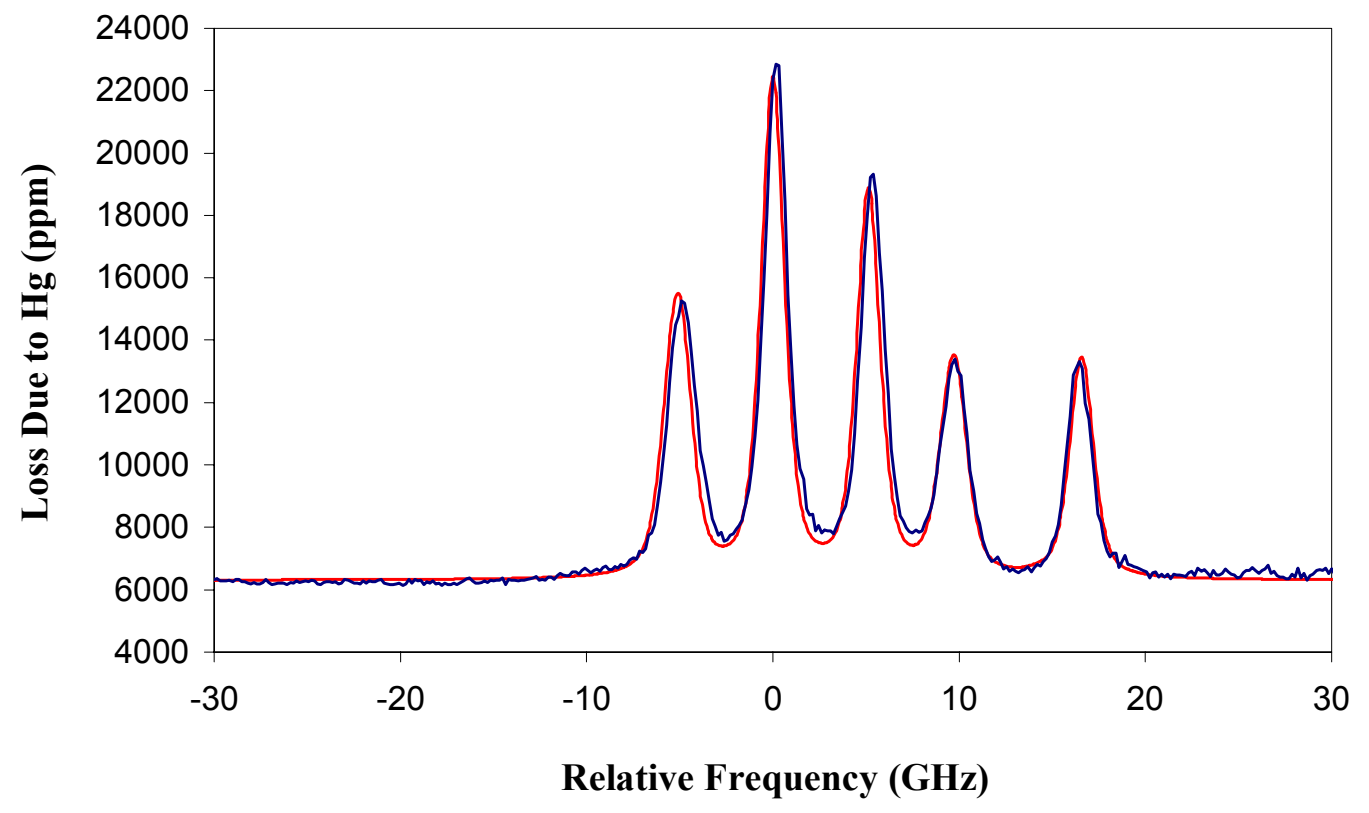

Figure 34: Plot of the relative frequency of the diode seed laser versus the losses in the cavity. The data was obtained with the cell maintained at room temperature, 90 torr pressure, and about $1.85 \mathrm{ppb}$ of mercury. The blue line is the experimentally obtained data and the red line is a theoretical plot fit to the parameters used in the experiment. 
As an additional check a second mercury source was tested. The plots shown in Figs. 33 and 34 were obtained with a mercury sample stream from a permeation tube held at $100^{\circ} \mathrm{C}$ in a temperature-controlled oven contained within a Dynacalibrator from VICI Metronics. A second permeation tube with a higher permeation rate, was held in a separate system from Environics, Inc., where the temperature controlled oven was maintained at $50^{\circ} \mathrm{C}$, was also tested with the new fiber optically coupled system. The results are shown in Fig. 35. The experimentally determined data is displayed with the blue line while the red line is the theoretical plot created with the experimental conditions used, namely the cavity held at room temperature, a pressure of 100 torr, and a mercury concentration of $6.2 \mathrm{ppb}$. This concentration is greater than 3 times that previously tested and again it can be observed from the plot that there is excellent agreement between the experimentally obtained data and the theoretically produced plot.

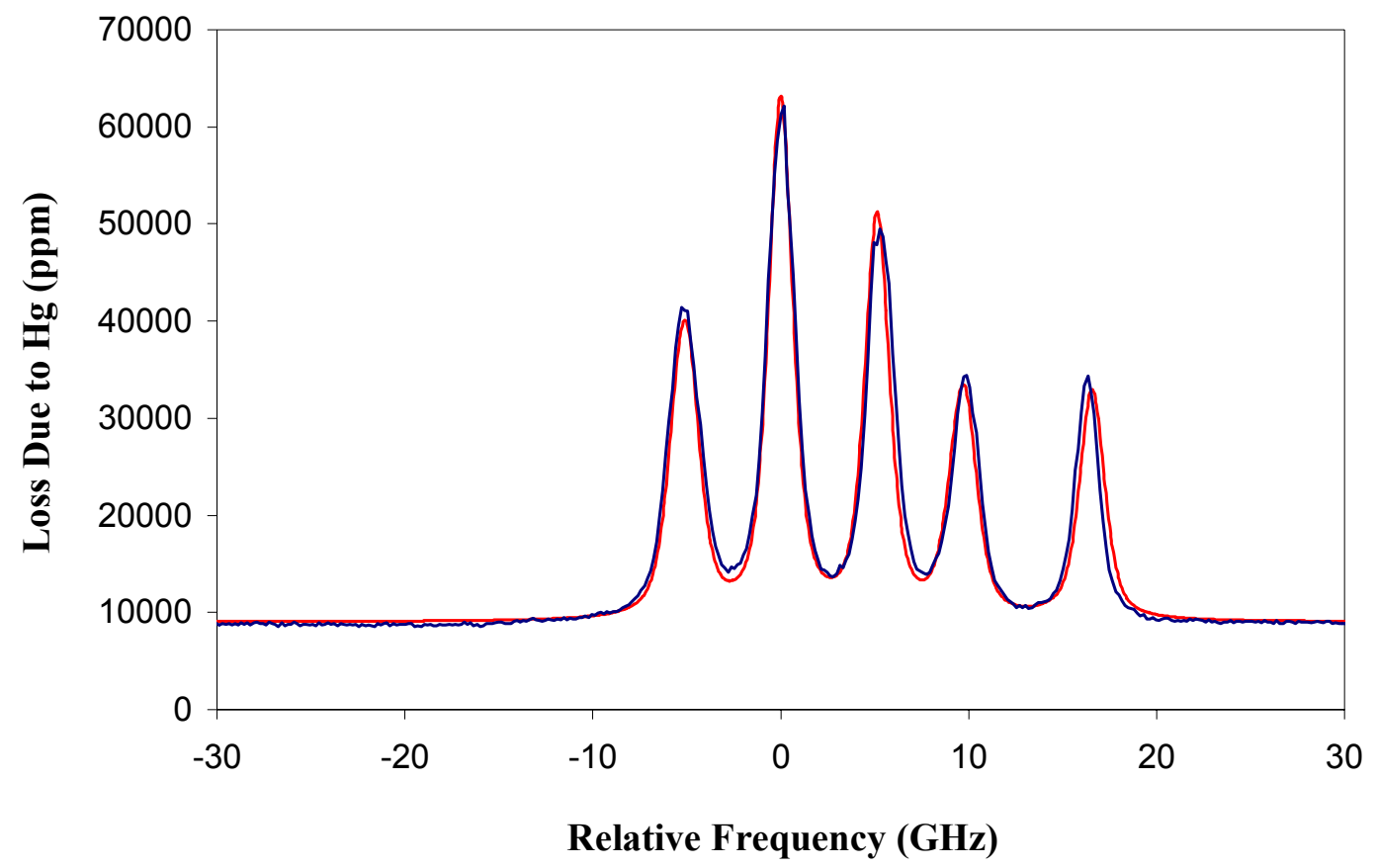

Figure 35: Plot of the relative frequency of the diode seed laser versus the losses in the cavity. The data was obtained with the cell maintained at room temperature, 100 torr pressure, and a flow rate of $1000 \mathrm{ccm}$ with 6.2 $\mathrm{ppb}$ of mercury. The blue dots are the experimentally obtained data and the red line is a theoretical plot fit to the parameters used in the experiment.

From the data shown in Figs. 33 - 35 it can be seen that the fiber optically coupled system appears to be operating exceptionally well. The amount of time that the fiber is in use transmitting UV radiation is being monitored to determine if there will be any long-term solarization effects on the fiber that might degrade the transmission characteristics over time. At this point there has been no degradation observed but it will be closely monitored. The power levels coupled into the fiber optic are in the very low to submillijoule per pulse range. This should also help to reduce the possibility of solarization of the fiber.

These results are promising for the transmission of the UV beam, through an optical fiber, over relatively large distances to remote monitoring sites where the mercury measurements will take place. 


\section{Sample System Construction}

Construction of the sampling system includes mounting everything needed, other than the laser system, onto a relatively small breadboard table. The breadboard table, including pyrolysis oven, CRD cavity, fiber optic coupling system, photomultiplier tube for ring-down detection, and the required tubing, valves, etc. is $3^{\prime} \times 4^{\prime} \times 2^{\prime \prime}$. The ability to fiber-optically couple the sampling system to a remote laser source significantly reduces the required footprint of the apparatus. A computer to acquire the CRD data, a pump used to flow gas through the system, power supplies, and temperature and pressure monitoring equipment, used to monitor the temperature of all the tubing and the temperature and pressure of the CRD cavity, will be located below the breadboard table. The flue gas supply and return line will be attached to the breadboard sampling system. Additionally, an environmental cover is being constructed to contain and protect all the elements of the CRD apparatus.

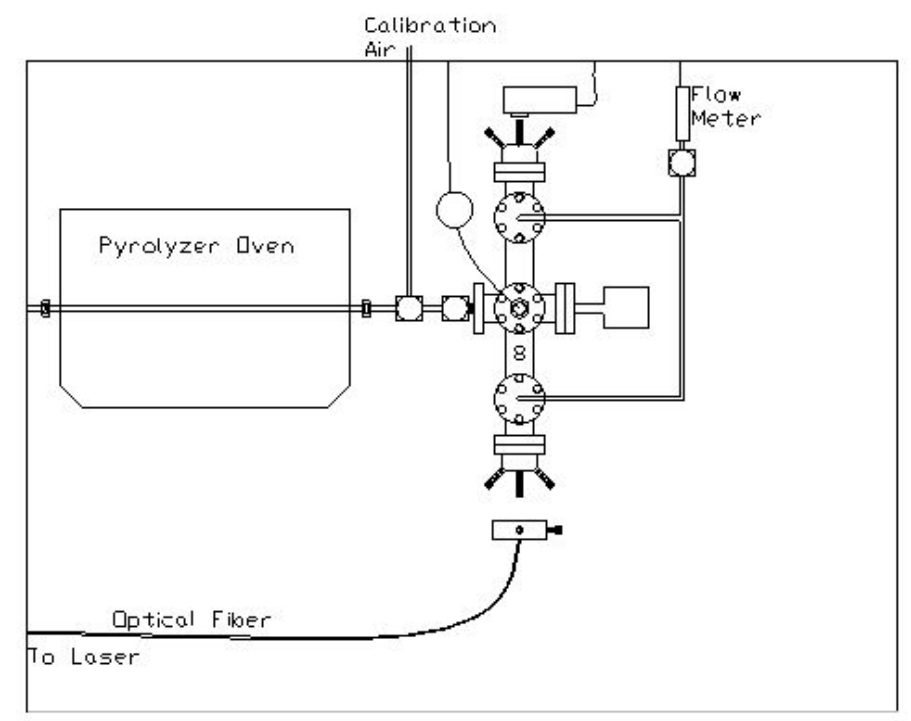

Figure 36: Diagram of the CRD sampling system coupled to the fiber optic carrying the UV laser pulse. The instrument is drawn on a $3^{\prime} \times 4^{\prime}$ breadboard table. All the equipment on the table is drawn to scale.

Figure 36 is a schematic drawing of the breadboard setup. All of the components have been drawn to scale, as has the 3' x 4' breadboard tabletop. The number of components and the amount of tubing has been minimized to reduce the resident time of the flue gas in the measurement apparatus and also to reduce the surface area available to the flue gas with which it could cause reactions and change the component mixture of the flue gas.

\section{Long-Term Studies}


The prime function of the CRD instrument will be to continuously monitor the concentration of mercury in a flue gas stream. To this end, SRD has tested the stability of monitoring mercury concentration over time with the CRD instrument under a variety of conditions. These conditions include using various pressures and temperatures. The pressure of the cavity is important as the linewidth of the various mercury isotopic transitions decreases significantly in going from 760 torr to 100 torr. Any frequency drift of the diode seed laser over time will accentuate apparent fluctuations in the mercury concentration, as the linewidth of the mercury transition gets narrower.

Shown in Fig. 37 is a plot of total cavity losses versus time. During this test the CRD cavity was kept at a pressure of 760 torr and a temperature of $200^{\circ} \mathrm{C}$. The mercury being delivered from the permeation oven source was maintained at a concentration of about $1.25 \mathrm{ppb}$ in air. The data points shown in Fig. 37 were obtained every 2 seconds over a period of $25 \mathrm{~min}$.

From Fig. 37 it can be seen that the concentration of mercury monitored by the Hg-CRD CEM varies only slightly over the 25-minute time span of the test. The standard deviation of the fluctuations over time was about $750 \mathrm{ppm}$ total cavity losses. These losses correspond to a standard deviation in the mercury concentration of about 30 pptr. The permeation oven is temperature controlled to $\pm 1^{\circ} \mathrm{C}$. SRD has performed a number of tests to determine the relationship between the temperature of the permeation oven and the mercury concentration. From this data it has been determined that fluctuations of $\pm 1^{\circ} \mathrm{C}$ would result in fluctuations of the mercury concentration of $\pm 10 \mathrm{pptr}$, assuming that the temperature within the oven has equilibrated. The permeation oven used for this data has a very small mass and the temperature within the cell could well vary by $\pm 1^{\circ} \mathrm{C}$.

While it appears that there are fluctuations above and beyond those attributable to just temperature changes of the permeation oven, they are relatively small. There are a number of possible sources, other than the permeation oven, of this variation over time. The fluctuations may be due to a slight frequency drift of the output wavelength of the diode seed laser over time, they may be due to shot-to-shot fluctuations of the laser system itself, or possibly fluctuations of the temperature and pressure at which the cavity was maintained.

Following the successful results shown in Fig 37, SRD undertook longer-term studies. Shown in Fig. 38 are the results obtained from a 2.5-hour study. The cavity was maintained at room temperature and a pressure of 760 torr. The mercury concentration was approximately $1.85 \mathrm{ppb}$, which was kept at a constant flow rate of $500 \mathrm{ccm}$ through the CRD cavity. The plot, in Fig. 38, is of the total cavity losses versus time. As can be seen from the plot there is very little deviation around the central loss level. The standard deviation obtained from this plot corresponds to a standard deviation in mercury concentration of about 35 pptr. Each point on the plot was obtained from the average of 200 laser shots, which required only 4 seconds to acquire the necessary data. 


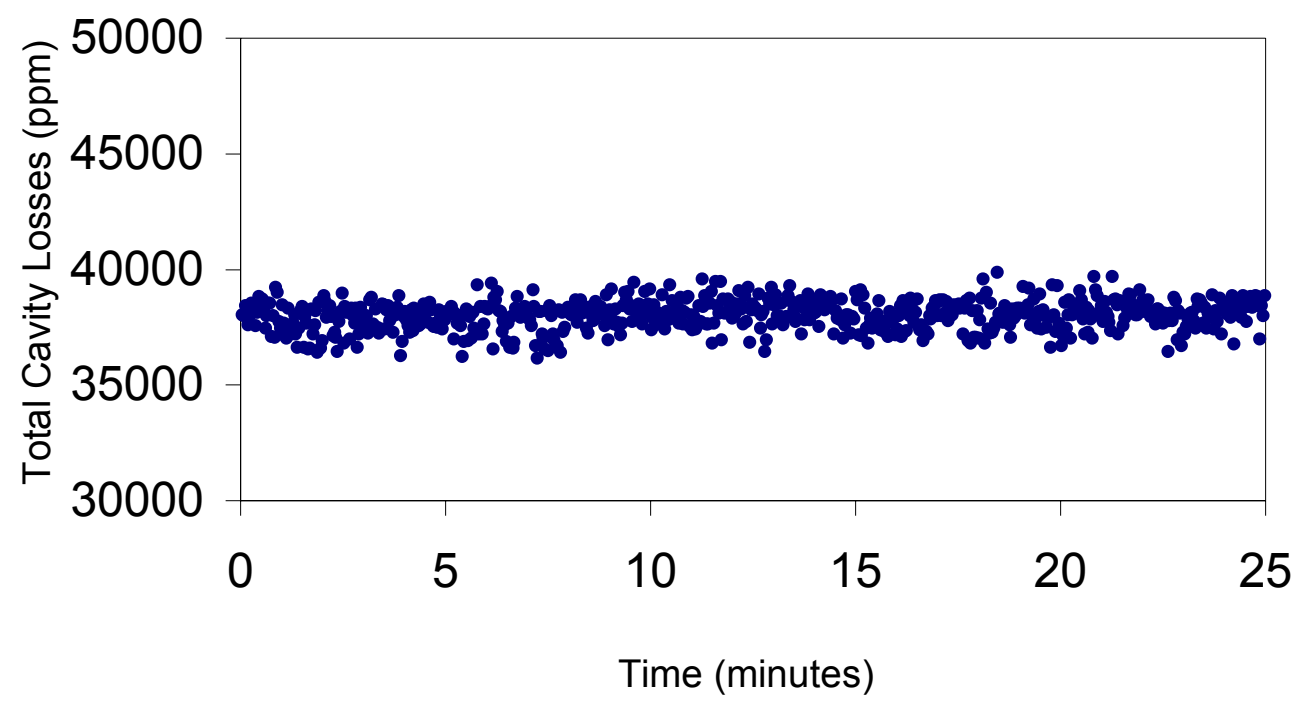

Figure 37: Plot of the cavity losses versus time. The data was taken with the $38 \mathrm{~cm}$ cavity that was held at atmospheric pressure (760 torr) and the temperature of the cavity was held at $200^{\circ} \mathrm{C}$. The concentration of mercury from the permeation oven was kept at $1.25 \mathrm{ppb}$ in air. A data point was acquired every 2 seconds. The standard deviation of the plot is about $750 \mathrm{ppm}$ total cavity losses, which corresponds to a mercury concentration of about 30 pptr.

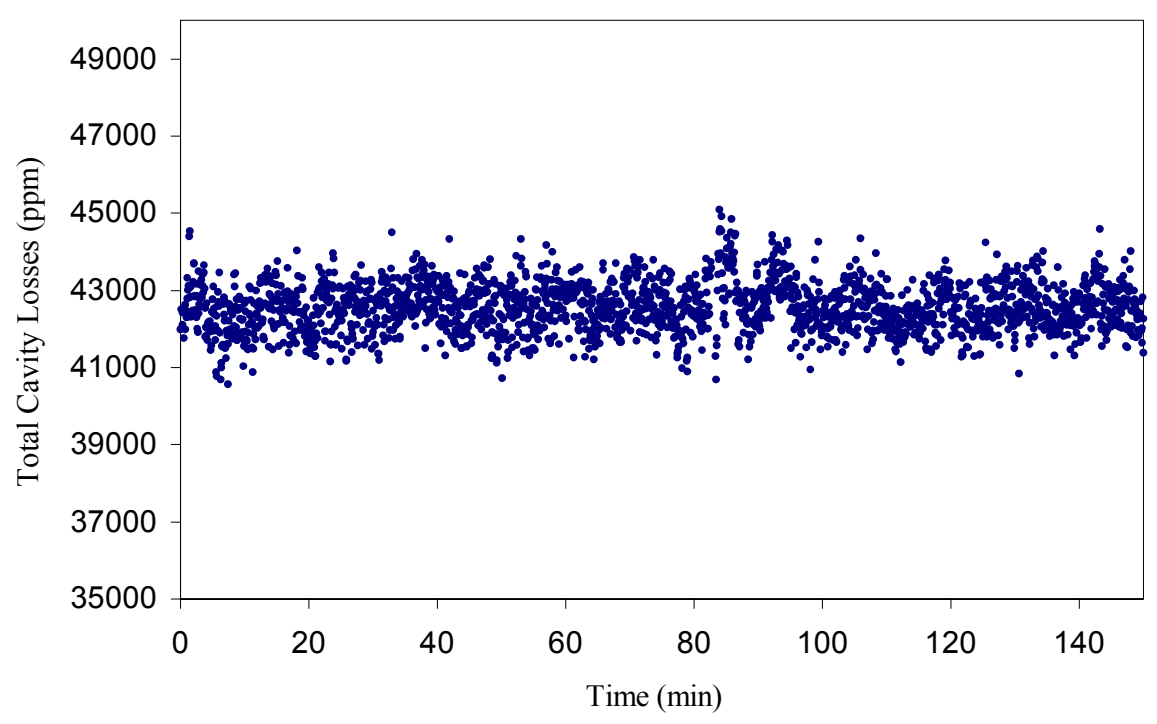

Figure 38: Plot of the cavity losses versus time. The cavity was maintained at room temperature, the pressure in the cell was held at 760 torr, and the mercury was constantly flowing through the cell with a concentration of 1.85 ppb. Each data point represents the average of 200 laser shots over a 4 second time interval.

In addition to the stability studies of the mercury concentration SRD investigated the time needed to reach the baseline level of the CRD instrument once the flow of mercury is cut off. The plot in Fig. 39 is one of total cavity losses versus time. The vertical line located at about 30 seconds into the plot is the point at which the mercury flow was stopped. This was done to investigate if there was any measurable mercury 
present in the system at times well after the flow of mercury had ended. The cavity was held at room temperature and a pressure of 1 atmosphere. The mercury concentration at time $=0$ was about $6.2 \mathrm{ppb}$ and the flow rate through the system was $1000 \mathrm{ccm}$. As can be seen in the plot the mercury signal quickly drops to the baseline level of the instrument and remains steady thereafter. The time period taken for the concentration to drop to baseline levels was consistent with the flow rate and the tubing and cavity volume of the CRD instrument present after the mercury permeation tube oven.

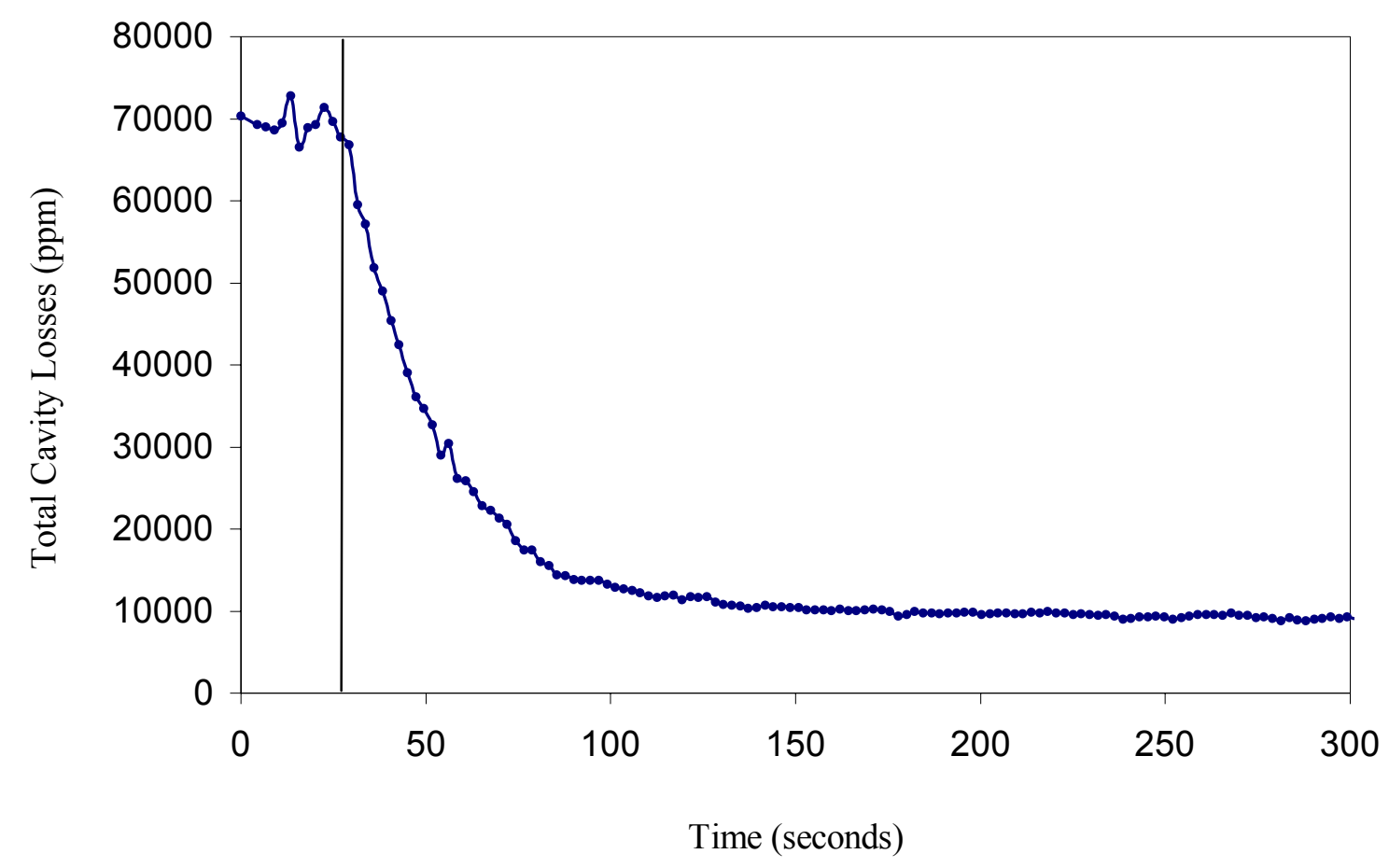

Figure 39: Plot of the cavity losses versus time. The cavity was maintained at room temperature, the pressure in the cavity was 760 torr, and the concentration of mercury at $t=0$ was $6.2 \mathrm{ppb}$. The vertical line on the plot indicates the time at which the mercury supply was shut off.

SRD was able to obtain the results shown above during the first attempt at longer term testing of the CRD instrument and its ability to monitor the concentration of mercury over extended periods of time. However, as these longer term studies were continued, by SRD scientist and engineers, there were a number of problems encountered in the ability of the CRD instrument to continuously monitor the mercury concentration over extended periods of time. A number of these findings, problems, and solutions are detailed below. 
Diode Laser Drifting

As the initial long-term testing noted above looked promising SRD sought to further these tests with a full 24-hour test. The results of the test are shown in Fig. 40. There was a continuous flow of approximately $2.5 \mathrm{ppb}$ mercury, from a permeation tube in a temperature-controlled oven, in air with the CRD cell held at room temperature and close to atmospheric pressure. From the plot it can be seen that the mercury concentration remained relatively level, with a few deviations around hours $0.5,3,7.5$, and 10.5 , for the first 14 hours. After about 14 hours there is a rather large decrease over 2 hours followed by a sharp increase back to the initial level. The plot again remains steady until about 22.5 hours after which there are a number of sharp changes in the observed cavity loss level.

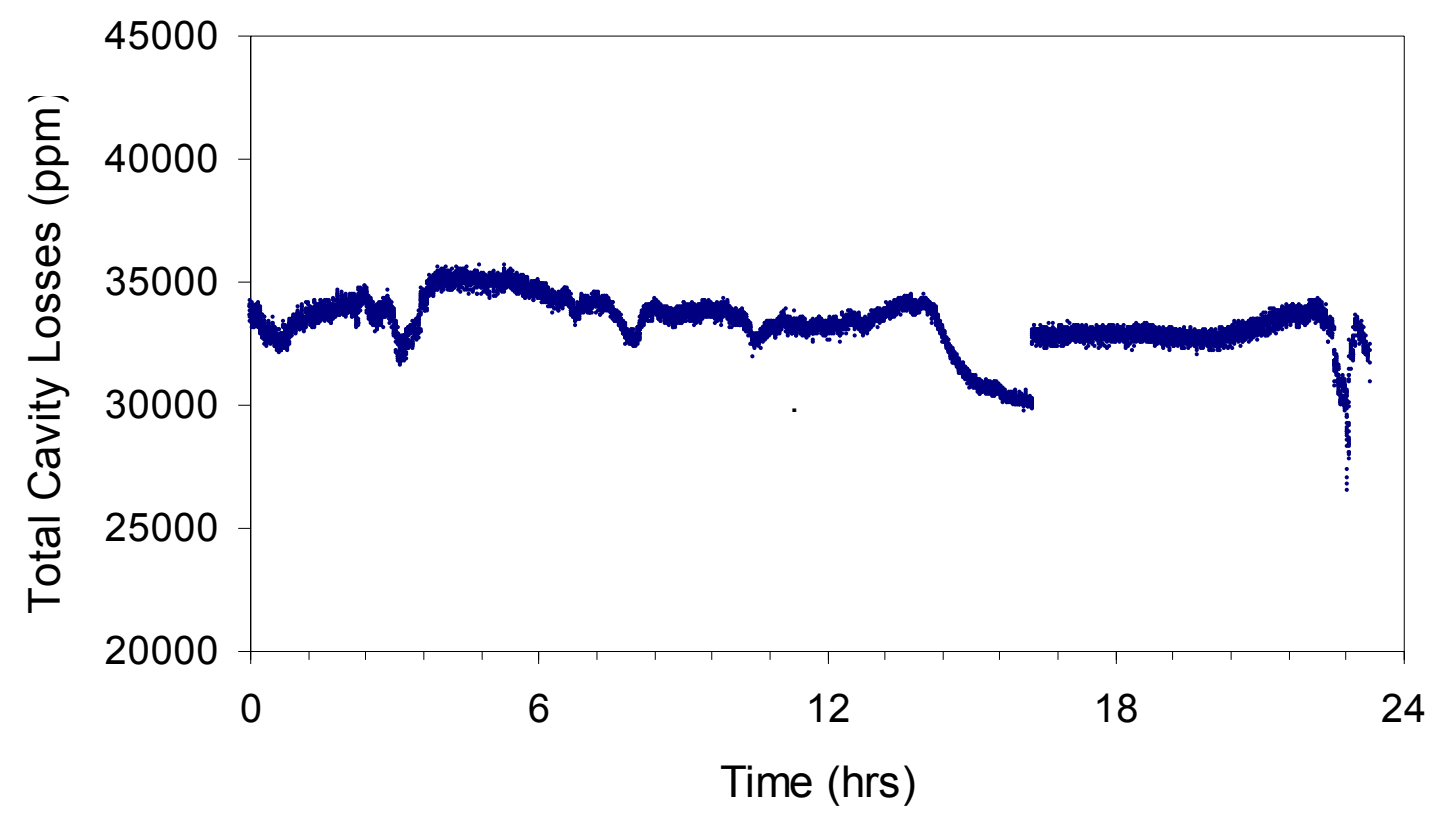

Figure 40: Results of a 24-hour test monitoring mercury concentration. The mercury concentration was held at approximately $2.5 \mathrm{ppb}$ in air while the cell was at room temperature and 730 torr.

There are two possible reasons for the observed deviations: 1) the mercury concentration is actually changing, which is reflected in the plot, or; 2) the frequency of the UV laser changes and is no longer sitting on the top of the mercury peak. The former is very unlikely as the mercury supply is from a mercury permeation tube that is contained in a constant temperature oven. Additionally, some of the changes occur over a time period that is too short to result from a change in mercury concentration. The only reasonable explanation is that the frequency of the laser that is injected into the cavity changes over time and results in the observed deviations.

The laser that controls the UV frequency output that is injected into the cavity is the diode laser that seeds the Alexandrite laser. The output frequency of the diode laser could be affected by something such as an internal temperature change within the housing of the laser itself. This drift in output frequency of the diode laser can be more clearly seen in Fig. 41. In this figure it can be seen that there is a regular 
oscillation of the diode laser on and off the top of the mercury peak. The observed oscillations in the figure correspond to approximately a $2 \mathrm{GHz}$ change in frequency in the UV. This corresponds to a change of about $650 \mathrm{MHz}$ of the diode seed laser, as it operates in the near infrared region, or $0.00125 \mathrm{~nm}$. The oscillations seen in Fig. 41 correspond to a change in mercury concentration of about 100 pptr. However, it should be noted that the observed changes are not always regular as was observed in the 24-hour test shown in Fig. 40.

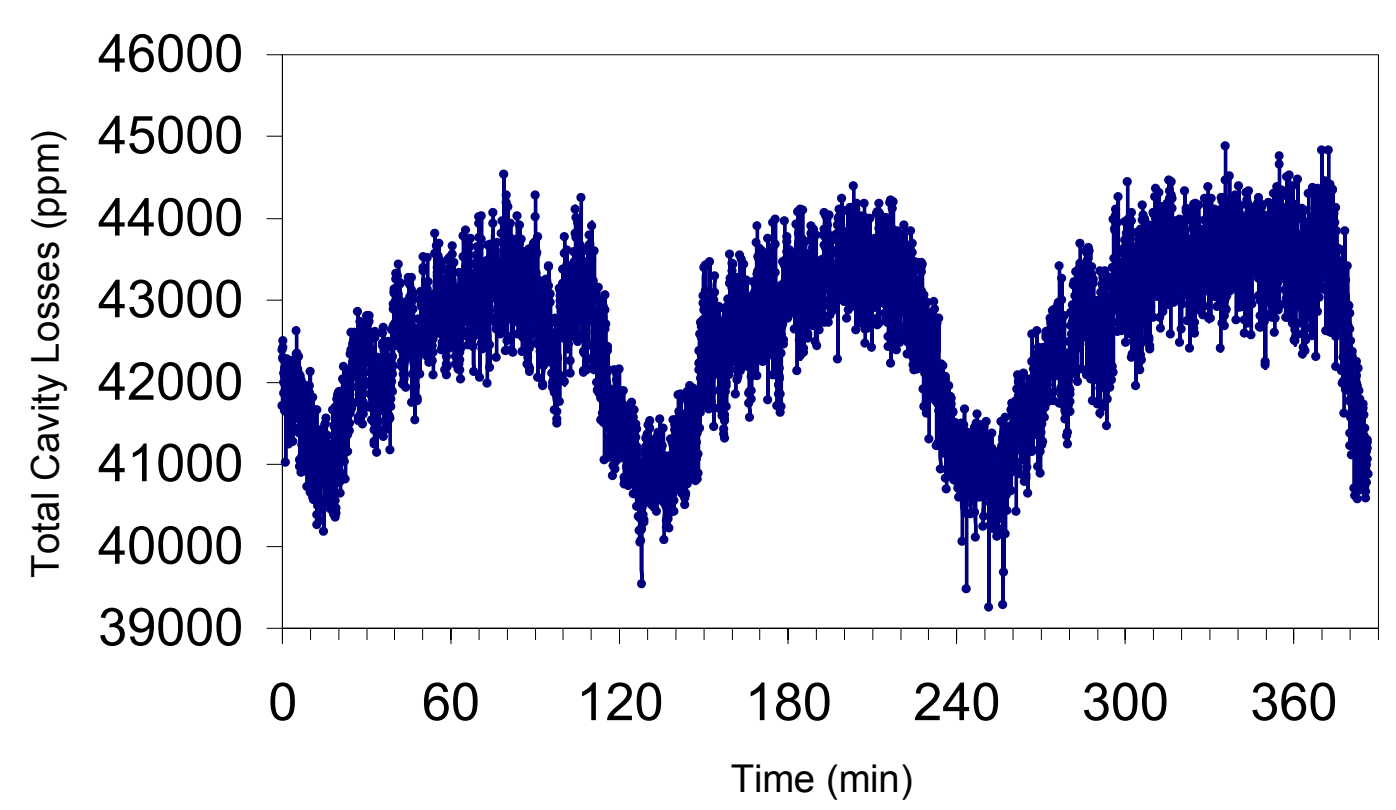

Figure 41: Plot showing the mercury concentration over time. The cell was kept at room temperature and atmospheric pressure. The mercury concentration was approximately $1.8 \mathrm{ppb}$. The observed oscillations are due to a frequency drift of the diode seed laser.

\section{Frequency Dithering}

The frequency drift observed when the laser is tuned to the top of the mercury peak is unpredictable, generally not regular, and unacceptable for the CRD instrument's monitoring of mercury concentrations. A possible solution to this problem is using the tunability of the diode seed laser to rapidly scan back and forth across the mercury peak. This would ensure that the value determined for the mercury concentration was obtained from the top of the mercury peak and not some position on the side of the peak resulting from a frequency drift of the laser.

Shown in Fig. 42 is a scan of the diode seed laser over the entire mercury transition. The ring-down cell was kept at room temperature and atmospheric pressure. The mercury concentration used was $1.25 \mathrm{ppb}$. Illustrated in the figure are two vertical lines spaced far enough apart in wavelength to cover the top of the largest isotopic mercury peak. These lines show the region over which the diode laser will be scanned back and forth. By calculating the derivative of each scan the maximum position, and hence top of the mercury peak, can easily be determined, or the maximum value obtained while scanning over the peak can be used. 


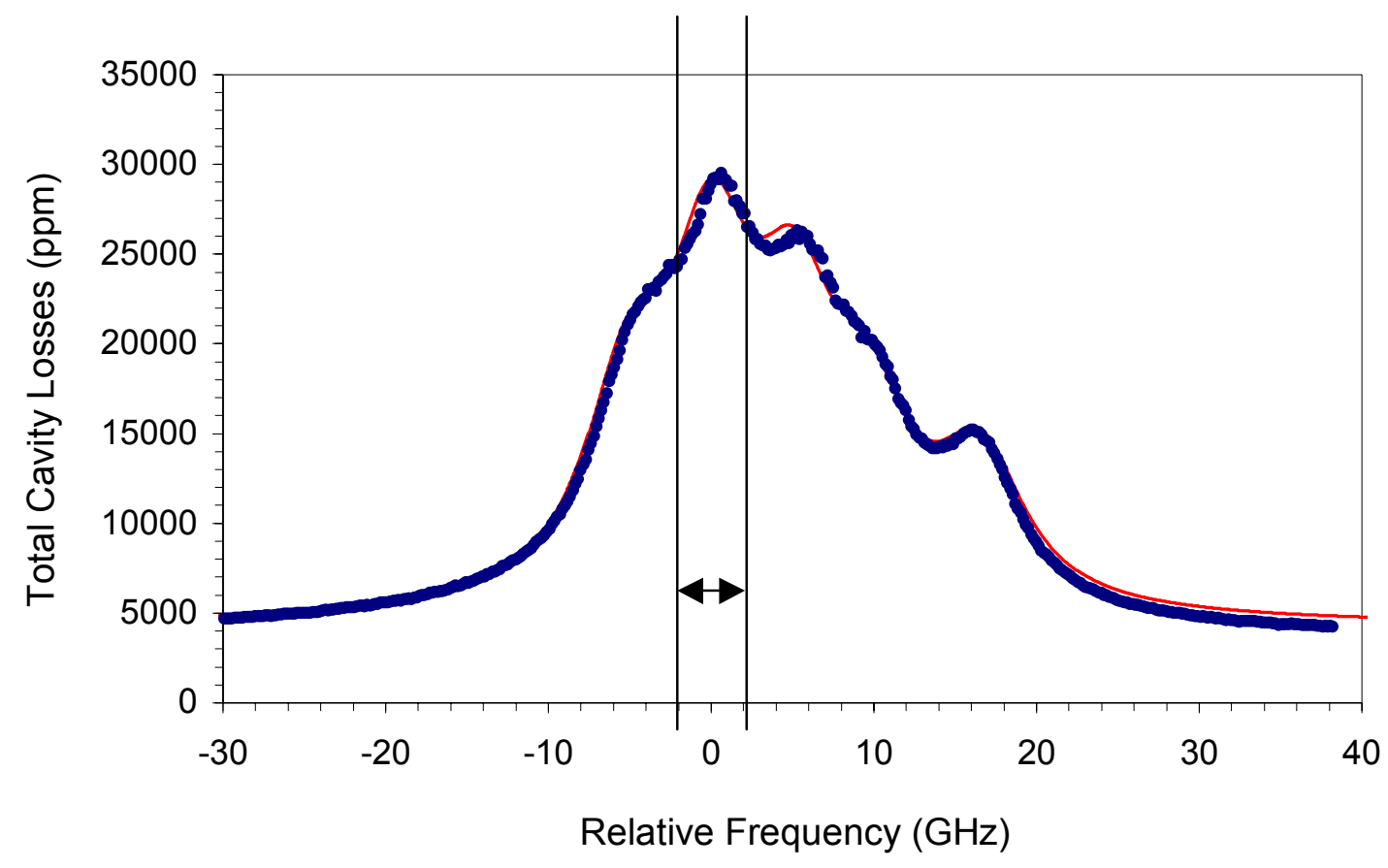

Figure 42: A scan of the laser frequency over the region of the mercury transition. The cell was held at room temperature and atmospheric pressure. The concentration of mercury was approximately $1.25 \mathrm{ppb}$. The vertical lines in the center of the plot shows the region that the diode laser was scanned back and forth across (see text).

From the plot shown in Fig. 42 it can be seen that the frequency region chosen to scan over was only 4 GHz. This is a relatively small frequency range and allows the diode laser to be scanned at a fairly rapid rate to determine the mercury concentration in a reasonable amount of time. This time period is dependant on not only the frequency range chosen but also the step size and the number of laser shots averaged for each point.

The plot shown in Fig. 43 is during the initial stages of a 5.5-hour test. The data obtained from dithering the frequency of the diode seed laser back and forth is illustrated with the blue line. The sinusoidal plot shows how the diode laser scans back and forth across the mercury peak as expected. The plot displayed in green is the derivative plot calculated from the obtained ring-down data (blue plot). From the derivative plot an inflection point (a maximum or minimum) is determined when the plot crosses the zero point of the y-axis displayed on the right side of the plot, which is shown by the horizontal black line located at the zero position. The red points shown on the plot display the all the maxima determined from the derivative data. From the plot is can be seen that the variation of the values of the red points is quite small. Indeed, shown in Fig. 44 is a plot of the maximum points determined from the derivative plot for the first hour of the test. The standard deviation for these points in the first hour is $271 \mathrm{ppm}$ total cavity losses. That translates to a standard deviation of the mercury concentration of $15 \mathrm{pptr}$, or about a factor of 8 less than that obtained from the data shown in Fig. 41. 


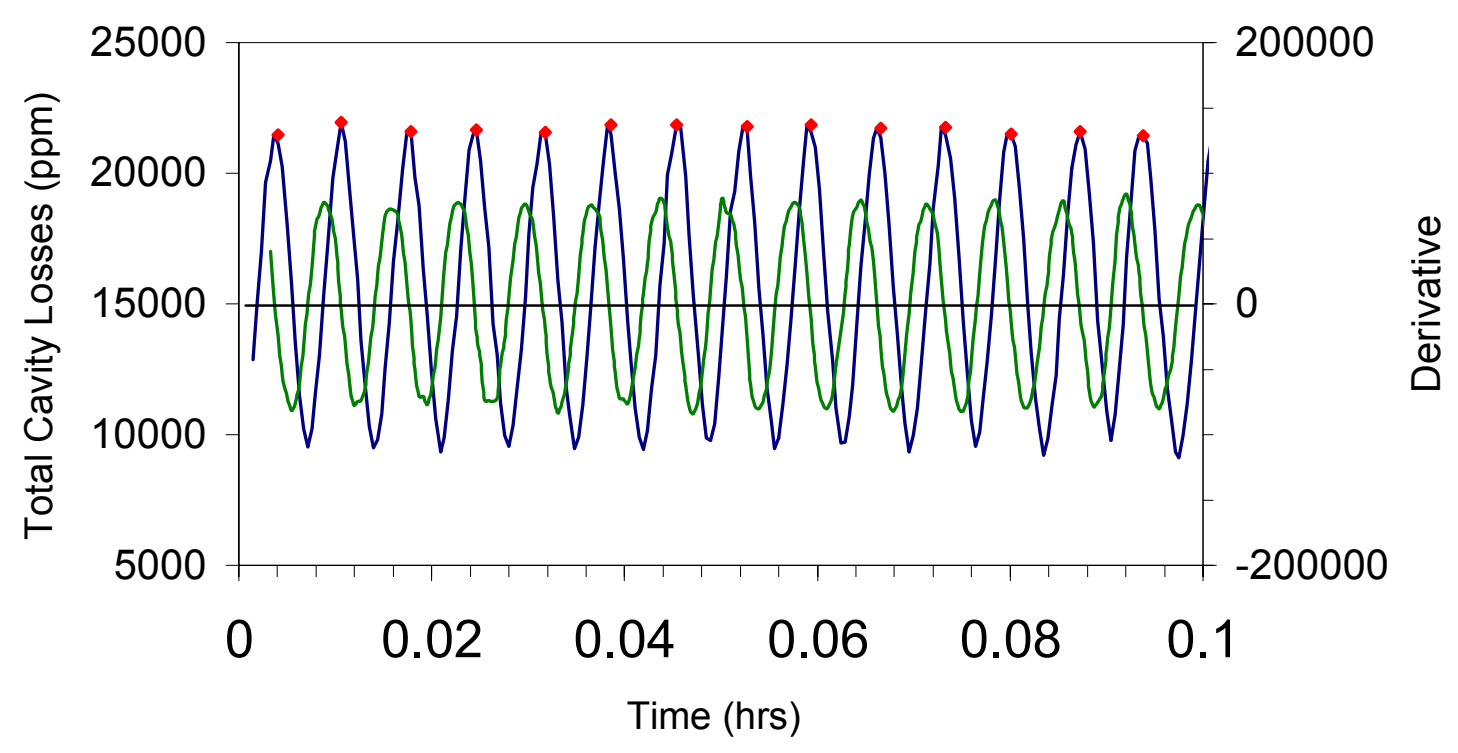

Figure 43: Frequency dithering of the diode seed laser across the highest mercury isotopic peak (blue data). The cell was maintained at $58^{\circ} \mathrm{C}$ and a pressure of 187 torr. The mercury concentration used was about $1.5 \mathrm{ppb}$. The derivative plot is shown in green and the maximum determined from the derivative plot is shown as the points in red.

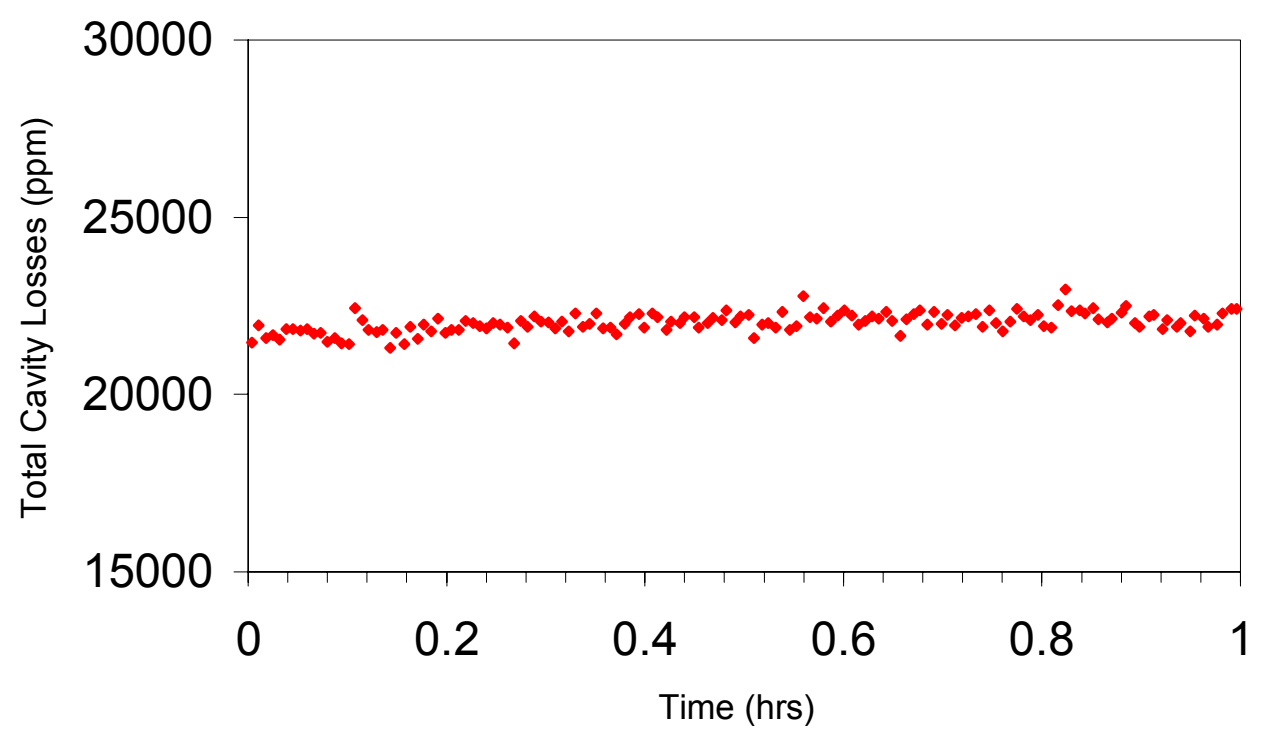

Figure 44: Plot of the maximum points determined for each scan across the highest mercury peak. These points are from the same plot that was shown in Fig. 43 but are shown for the first hour of the 5.5-hour test. 
The conditions used for the data displayed in Figs. 43 and 44 were such that the time taken to obtain each of the red points was less than 30 seconds. The initial stages of this test looked very promising. However, as shown in Fig. 45 these results did not hold for the entire test. From the plot it can be seen that there were a number of maxima obtained around 1.5-hours into the test that are at a loss level of about 7000 ppm when it should be around $22000 \mathrm{ppm}$. Additionally, after 4-hours of testing a sharp decrease occurs followed by a number of other sharp changes, which eventually return to the maximum level.

The low values for the maxima obtained around 1.5-hours into the test occur because of a small peak that appears when dithering the frequency of the diode seed laser back and forth. The sharp changes that occur after 4-hours into the test are most likely the result of a mode hop in the frequency of the diode seed laser. This means that another mode within the diode laser starts to have an appreciable amount of gain to the point that the frequency of the output jumps from one wavelength to another, resulting in the observed sharp changes.

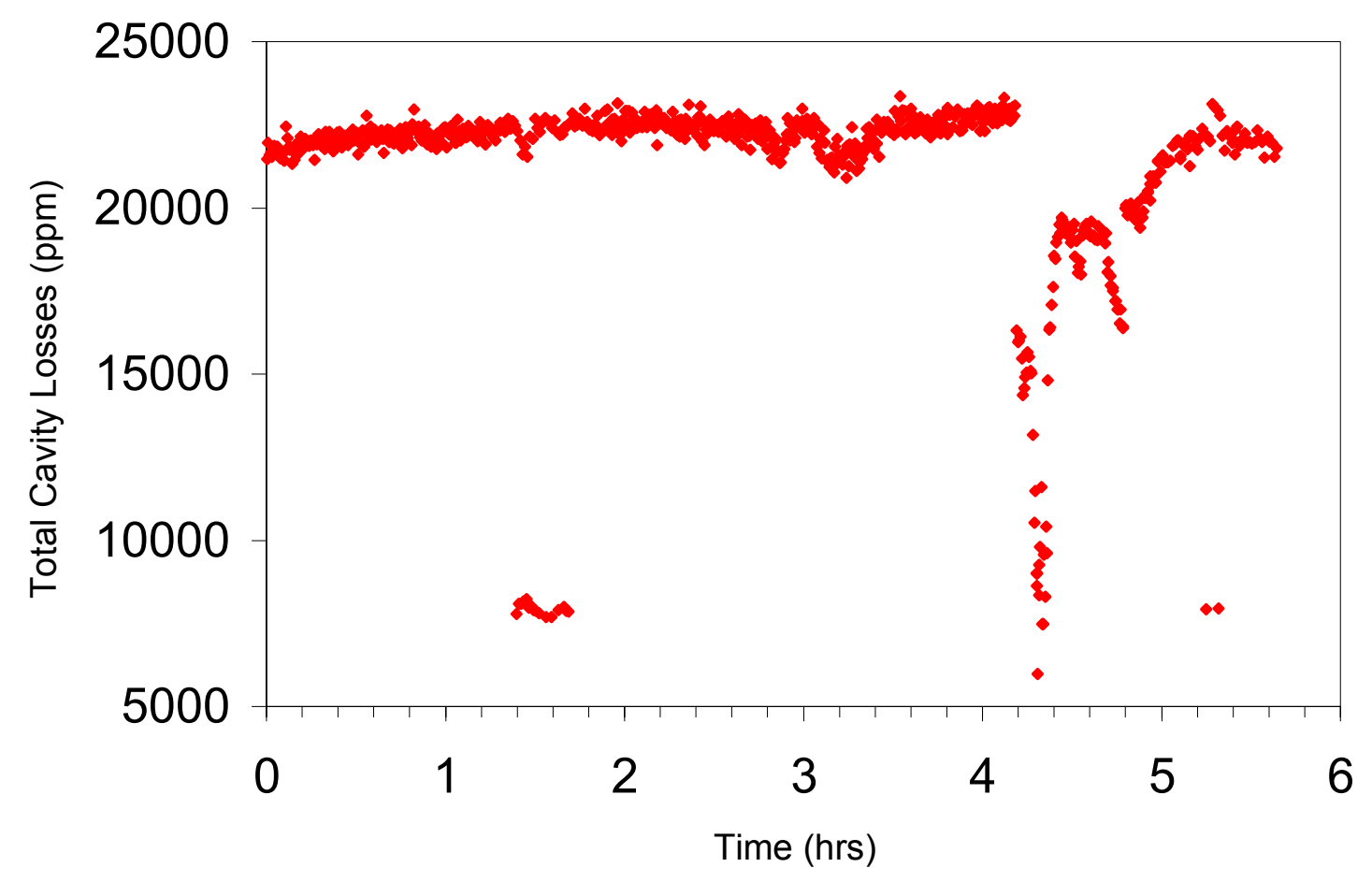

Figure 45: Plot of the maximum points determined from the derivative of the ring-down data shown in Fig. 43. The plot displays all the maximum points obtained for the entire 5.5-hour test. 


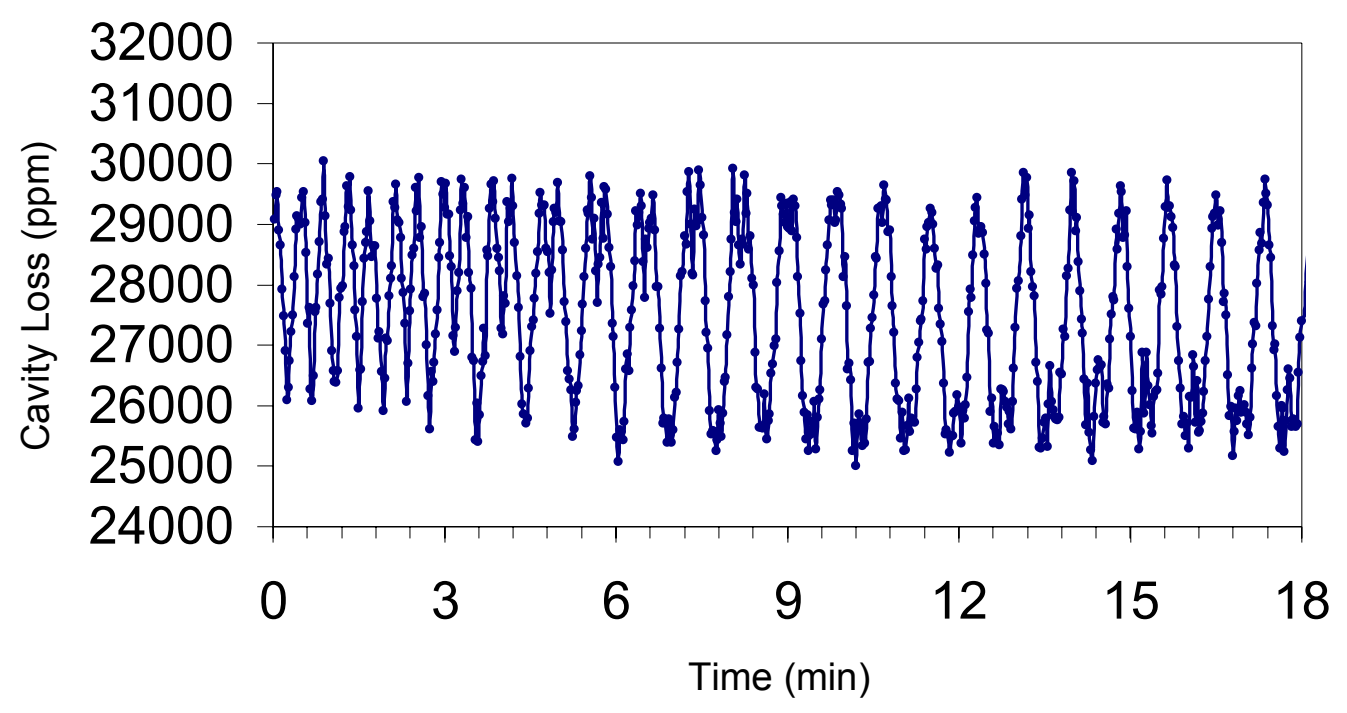

Figure 46: Spectrum observed from dithering the frequency back and forth over a single mercury isotopic peak.

Further investigation into the small peaks that occur near the minima, resulting in the low points observed in Fig. 45 around 1.5-hours into the test, revealed continued drifting of the diode laser. This phenomenon is illustrated in Fig 46. Another scan dithering the diode seed laser back and forth was performed and it can be seen that the first 3 minutes show a constant and repeatable dithering back and forth evenly across a mercury peak. However, from minutes 3 to 10 one can observe that the frequency of the diode laser is drifting as it is dithering. The wavelength slowly drifts off the peak until the region scanned no longer goes across the peak, although it still reaches a level very close to or just on top of the mercury peak. The drifting continues through minute 18 where another peak can be seen emerging at the minimum position of the scan. This peak is one of the other isotopic mercury peaks located spectrally adjacent to the highest isotopic peak and would result in erroneous maxima positions determined automatically by the CRD software, as observed in Fig. 45.

\section{Software Solutions}

One solution to the above mentioned problems, of both drifting of the output frequency of the diode seed laser as well as mode hops, is adding a couple of subroutines to the LabVIEW software that already controls the laser as well as the data acquisition of the CRD instrument. SRD software engineers have put together a few subroutines to deal with the problems of the drifting laser frequency as well as the problems with mode hops.

The new LabVIEW subroutines are designed to scan the laser wavelength over or near the top of a single mercury isotopic peak. The program determines the maximum loss value from the derivative, as described above and depicted in Fig. 43. If the maximum value is at the beginning or end of the scan it is not the absorption peak center and the scan center is reset to this value. This continues until the maximum value is at the center of the scan. This maximum value is the center of the mercury absorption peak from which the concentration is determined. Due to the slowly varying laser wavelength, caused by drifting of the 


\section{Sensor Research \& Development Corporation DE-FC26-01FT41221}

diode seed laser, the peak value is re-centered after each scan. This re-centering ensures that the mercury peak will continue to be located at the center of the dithering scan range. This can be done for any of the 5 different isotopic peaks.

The problem of mode hops is somewhat more complicated. A mode hop can change the output wavelength to either a higher or lower wavelength. As a result the actual wavelength will be unknown and the re-centering subroutine could eventually lock onto the wrong isotopic peak and report incorrect concentration values. To deal with this error an additional subroutine has been written that will reset the laser to a position off resonance from which the laser will be scanned back over the mercury transition. The individual isotopic peak separations are well known, as are the relative peak heights, and once this pattern is established the laser will again be able to relocate to the proper mercury peak. Once relocated the program will revert to the above-mentioned subroutine and continue dithering back and forth to report the correct mercury concentration.

There are a number of parameters that have been built into each subroutine to maximize the performance of the CRD instrument as well as minimize the time needed to locate the desired mercury peak and begin determining the absolute mercury concentration. Some of these parameters include the number of shots averaged at each point, the scan range used for dithering the laser over the mercury isotopic peak, the temperature and pressure of the cavity itself, and the time duration used between checks of the baseline level of losses in the cavity. These are just a few of the many parameters that can be used to maximize the performance of the CRD instrument.

One of the software windows used for data acquisition with the CRD instrument is shown below in Fig. 47. This window is the main window for checking the ring-down curves, the accuracy of the fit to an exponential decay, and scanning the Alexandrite laser system versus frequency (voltage to the diode seed laser) or time. There are a number of graphs displayed in Fig. 47. The small plot in the middle of the left side is a display of the individual ring-down decay for each laser shot, while the top left graph is a display of the averaged ring-down decay for the desired number of laser shots. The plot shown on the bottom left of the panel is the results of the exponential fit. The white dots are the experimentally obtained data and the red line is the best fit determined in the LabVIEW program. The determined total losses of the cavity, ring-down time, and resulting errors of the fit are shown just to the right of the graph. The graph displayed in the top right of the window is an optional channel for a second set of data obtained simultaneously. The plot in the lower right of the panel is the display of the results that plots total cavity losses versus frequency or time. Included on this panel are a number of buttons along the right hand side that allow the user to set a number of parameters used during a run such as: number of shots averaged, frequency (voltage) scan range, starting and ending point, and the gates to be used to determine the region of the ring-down decay to fit.

The second panel, shown in Fig. 48, displays additional functions of the LabVIEW program that controls the CRD instrument. In this window the graph displayed at the bottom is used to find the top of the desired mercury isotopic peak. Any one of the five observable isotopic peaks can be chosen to monitor the mercury concentration. Once the desired peak is chosen and found by the program the software initiates frequency dithering across that desired peak. As the diode seed laser is scanned back and forth across the peak it is recentered after each individual scan. After each scan the peak value for the losses within the cavity are found and the concentration of mercury is determined. The graph shown at the top of the window is a display of the mercury concentration versus time. 


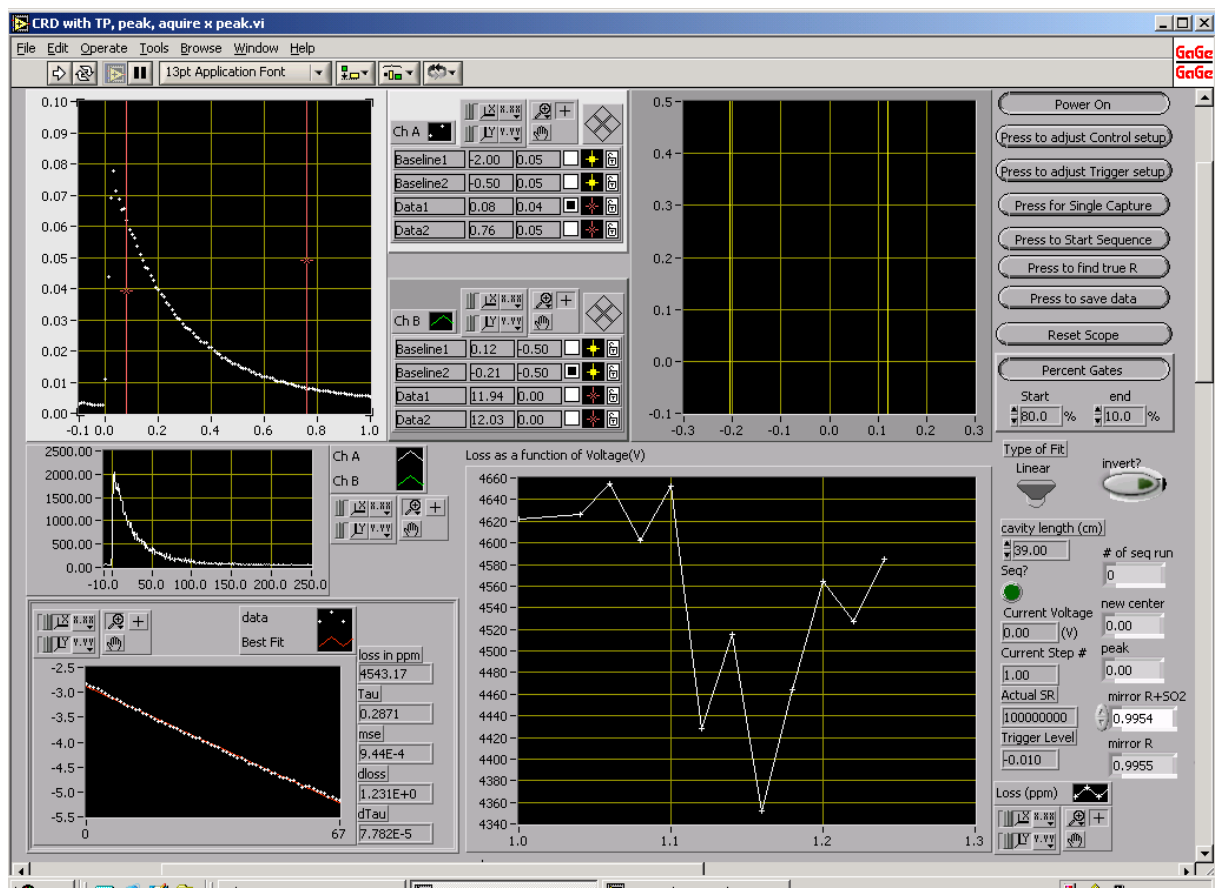

Figure 47: One of the windows available with the LabVIEW software used for obtaining CRD data. The window depicts a number of the options available and functions of the software (see text).

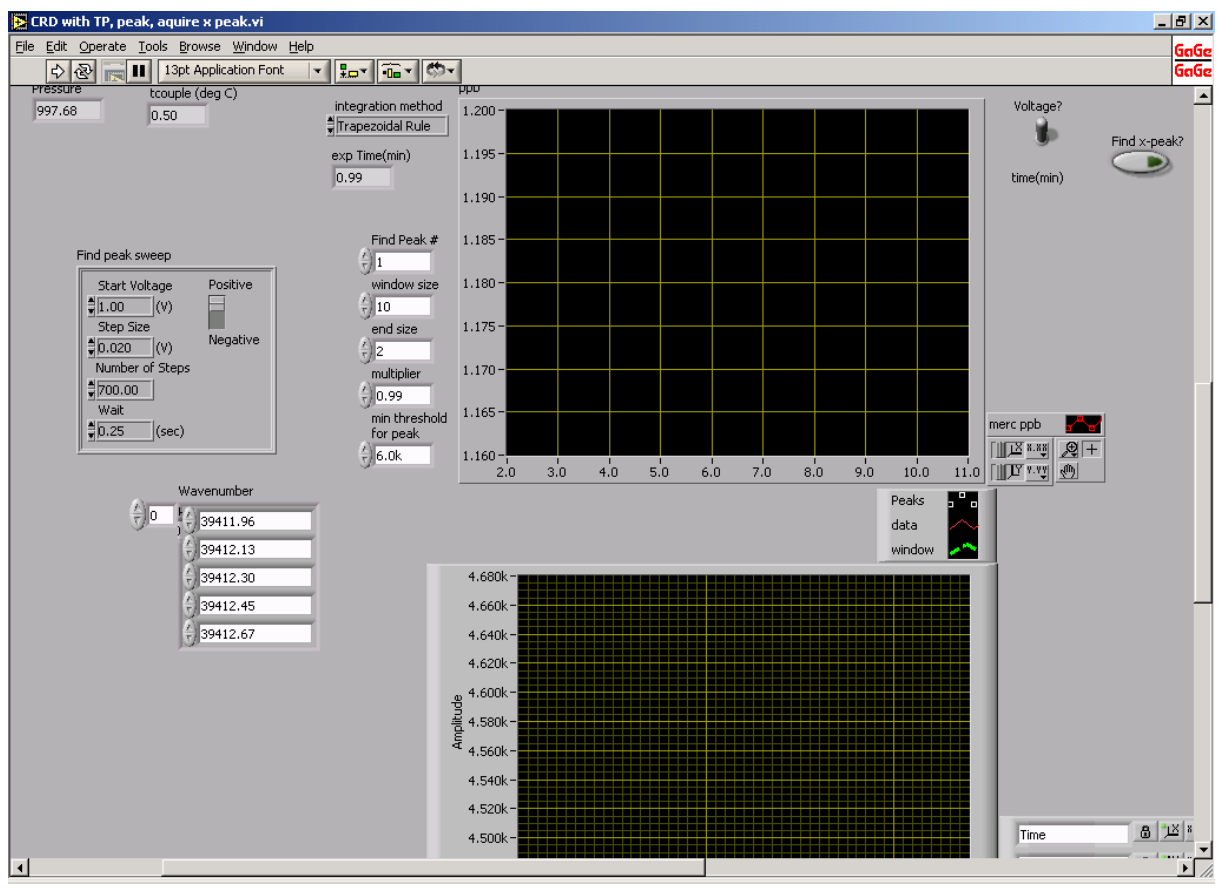

Figure 48: A second window used in the LabVIEW software for the CRD instrument. This window is used for finding a particular isotopic mercury peak to be used to monitor the mercury concentration.

When these changes were implemented another long-term test was attempted. The results of this test are shown in Fig. 49. This test was run for almost 3 consecutive days, 24 hours a day. The concentration of 
mercury was held at $60^{\circ} \mathrm{C}$ and the pressure of the cell was maintained at 200 torr. The results were extremely good. There were only two areas that showed deviations from the correct concentration. That was 6 points between 30 and 40 hours into the test and also around 60 hours into the test.

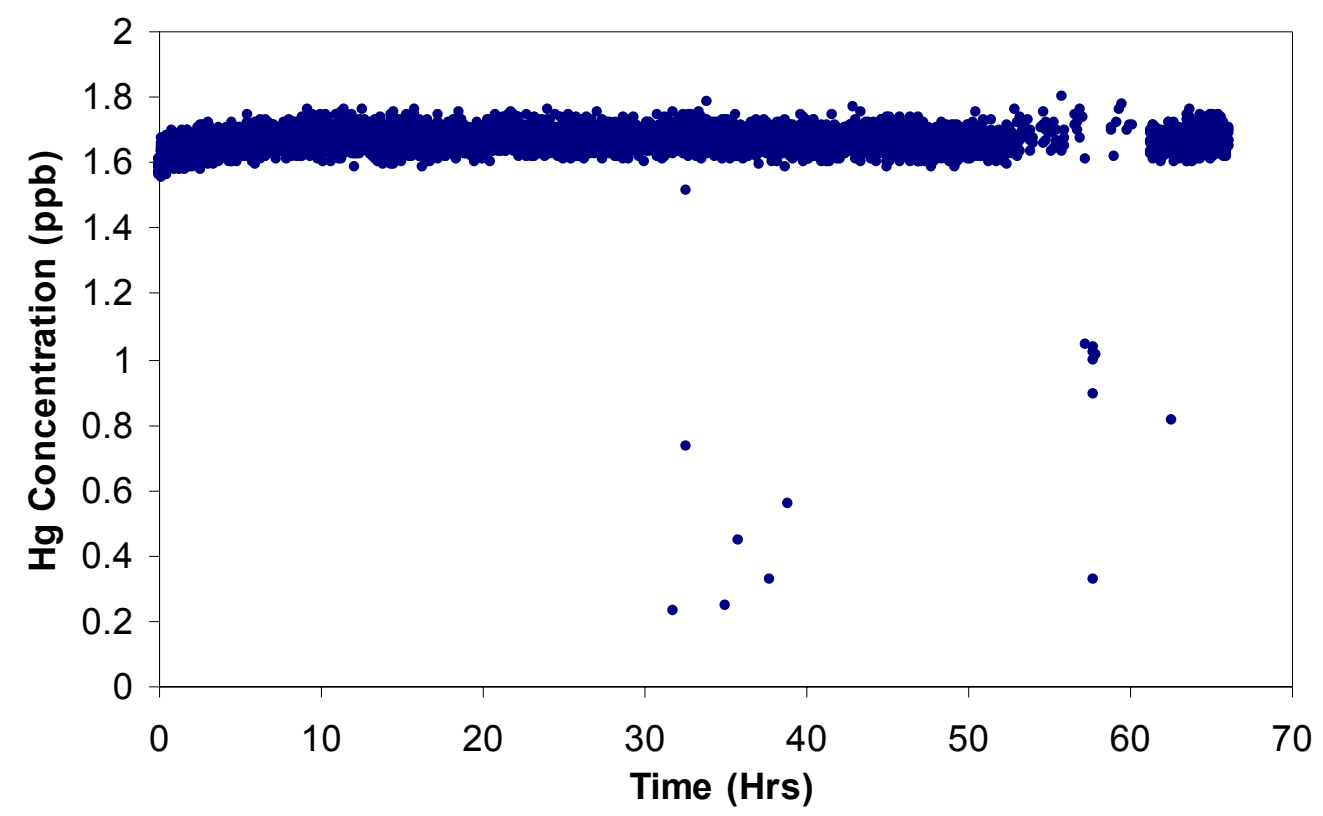

Figure 49: Plot of an almost three day test. The mercury was held at a concentration of about $1.6 \mathrm{ppb}$, the temperature of the cell was maintained at $\sim 60^{\circ} \mathrm{C}$, and the pressure in the cell was 200 torr. The ${ }^{202} \mathrm{Hg}$ isotopic line was used to monitor the concentration.

The points located around 60 hours were easy to explain. The power of the UV laser beam had dropped below the threshold value to trigger the software, which resulted in the slight gap and erroneous points. Once the power of the laser was increased the reported concentration returned to where it should. Of more interest were the points located between 30 and 40 hours into the test.

Figure $50 \mathrm{~A}$ ) is a blowup of the section between 30 and 40 hours. Figure $50 \mathrm{~B}$ ) is a plot of the voltage determined for the monitored mercury peak center. From the plot of the peak center voltage it can be seen that there is a one-to-one correspondence between sharp changes in the peak voltage value and the erroneous mercury concentration point, as shown for the first two point by the red circles and arrows. This figure actually illustrates both problems of drifting and mode hops. From the plot of the peak voltage it can be seen that the peaks voltage drifted down in value a significant way before there was a sharp increase in the voltage. This sharp change is when the mode hop occurred. At this point the program determined the mercury concentration point was erroneous, reacquired the correct mercury peak and resumed scanning back and forth across the peak. The voltage then drifted to higher values for about an hour and then mode hopped again. This was repeated three times. The exact cause of this behavior is unknown at this time. However, it should be noted that the program independently detected the mode hop and reacquired the correct mercury peak and reported the correct mercury concentration. While the points are reported during this test it is for diagnostics purposes only. For actual results these erroneous points would not have been reported as the program was reacquiring the correct mercury transition to monitor the concentration. 


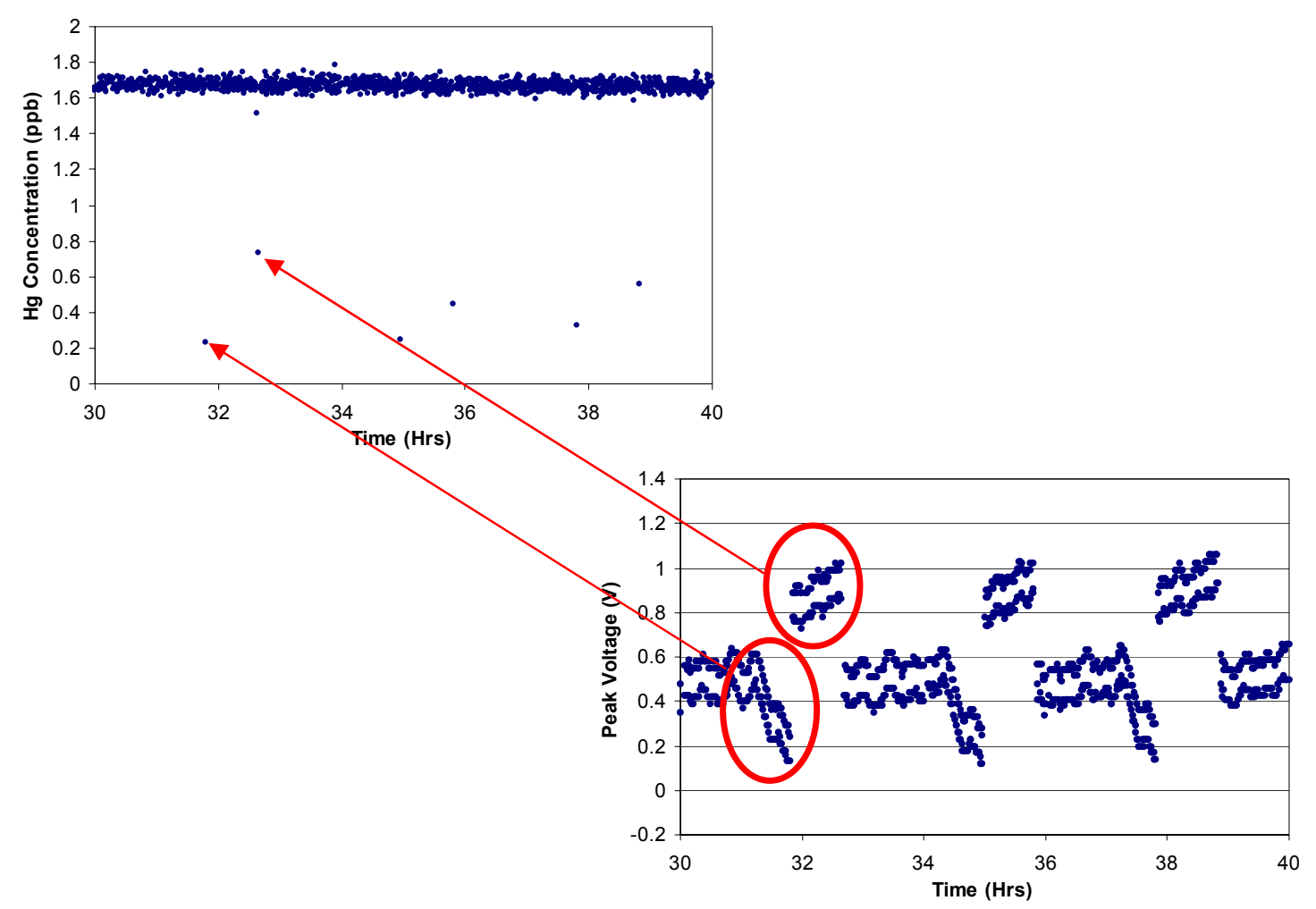

Figure 50: A) Expanded plot of the 30 to 40 hour section of the plot shown in Fig. 49. B) Plot of the voltage value, supplied to the diode seed laser, where the program determines the center of the mercury transition to be.

\section{Mercury Calibration and Verification Procedures}

The CRD instrument reports absolute concentrations of mercury. The losses determined from the ringdown time can be correlated to the total number of mercury atoms within the sample pathlength contained in the CRD cavity. However, to verify that there were no problems with the program or the reported concentrations additional methods were needed to verify the concentrations of mercury delivered to the CRD instrument.

SRD scientist and engineers verified the concentrations delivered to the CRD cavity using a couple of different methods. The first was by using mercury permeation tubes with a specific permeation rate to deliver the mercury, and the second was to use an Atomic Fluorescence Spectrometer (AFS) to verify the mercury concentrations delivered at any point throughout the CRD instrument's sampling system and ring-down cavity. 


\section{Mercury Permeation Tubes}

The mercury permeation tubes used for the duration of this project were obtained from VICI Metronics, a division of Valco Instruments Company Inc. The permeation tubes have a specific permeation rate for a specific temperature. A number of different tubes with a variety of permeation rates were used throughout the project.

SRD used two different temperature controlled ovens for sources of mercury. One was a gas delivery system with temperature-controlled ovens from Environics Inc., and the second was a Dynacalibrator from VICI Metronics. Both systems had temperature-controlled ovens that would maintain the set temperature to $\pm 1^{\circ} \mathrm{C}$. The Dynacalibrator contains a much larger oven than the Environics system and ended up being the mercury delivery system of choice.

Once the specific permeation rate is known adjusting the flow rate of the sample carrier gas can change the mercury concentration to a set level. The mercury concentration can be easily calculated by, ${ }^{18}$

$$
C=\frac{P\left(\frac{24.46}{m w}\right)}{F_{C}}
$$

where, $C$ is the concentration of mercury in ppm, $P$ is the permeation rate in $n g / m i n, m w$ is the molecular weight of mercury, and $F_{C}$ is the flow rate of the sample carrier gas. The flow rate is assumed to be at standard temperature and pressure. Should the temperature and/or pressure change the changes should be reflected in an adjusted flow rate.

The permeation tubes are rated at a certain permeation rate at one specific temperature. However, if the temperature of the oven is changed the permeation rate can be adjusted to reach an even wider range of mercury concentrations. The relationship between temperature of the tube and permeation rate is given by, ${ }^{18}$

$$
\log P_{1}=\log P_{0}+\alpha\left(T_{1}-T_{0}\right)
$$

where, $P_{0}$ is the initial permeation rate at temperature $T_{0}, P_{l}$ is the new permeation rate at temperature $T_{l}$, and $\alpha$ is a temperature coefficient that is specific for either high or standard emission permeation tubes. As a general rule-of-thumb for every $1{ }^{\circ} \mathrm{C}$ increase in temperature the permeation rate increases about $10 \%$.

The permeation rates of the mercury tubes, at the rated temperature as well as other temperatures, were verified with SRD's Atomic Fluorescence Spectrometer.

\section{Atomic Fluorescence Spectrometer}

The Atomic Fluorescence Spectrometer (AFS) employed at SRD was a Brooks Rand Model III. As mentioned above this was used to verify the permeation rates of the different mercury permeation tubes that were used. In addition to verifying the permeation rates directly out of the temperature controlled 
oven, measurements were made, on a continuous basis, at a variety of points along the sample stream to ensure that there was no mercury loss through any of the components of the CRD instrument.

The AFS was calibrated with a specific procedure using headspace measurements from mercury contained in a temperature-controlled bath. Details of the procedure are given below. This calibration procedure was done on a daily basis when the CRD instrument was being tested with specific mercury concentrations. Once calibrated mercury samples were taken in-line with the sample train at the exit port of the temperature controlled oven, the input of the ring-down cavity, and the exit of the ring-down cavity to ensure no mercury losses within the sample stream or cavity itself.

The procedure outlined below is the method that was used for the acquisition of $\mathrm{Hg}$ headspace calibration measurements. These calibration measurements are used to create a calibration standard of elemental $\mathrm{Hg}$ in the gas-phase, which is subsequently used to quantify $\mathrm{Hg}$ in gas samples taken from other sources.

\section{Equipment}

The following equipment is used to acquire the headspace measurement data.

- Atomic Fluorescence Spectrometer, Brooks Rand Model III

- Temperature controlled bath, VWR model 1162

- Syringe, $2.5 \mathrm{ml}$, Hamilton series 1000 Gastight model 1002 with side port point style \#5

- $\quad$ Multimeter, Keithley model 2000

- Gold wire trap with Nichrome heater filament, Brooks Rand p/n AF-19

- Mass flow meter, 500 sccm, Cole Parmer model 32915-86

- Teflon tubing and fittings, $1 / 4 "$ ", in quantity sufficient to make interconnections between the argon cylinder, the trap, the AFS, and the exhaust filter and the laboratory exhaust gas port.

- DC regulated power supply with interconnect wiring, 0-20 VDC, 10 amp, MG model PS10AD.

- Computer, capable of supporting Brooks Rand AFS sampling software (Mercury Guru) and SRD proprietary program.

- Database, Microsoft Excel, SRD compiled Excel file used to perform calculations and store relevant data.

- Charcoal filter, Gelman Sciences p/n 12011.

- $\quad$ Resistive temperature device (RTD) Omega model F3105, with wiring to multimeter.

- Charcoal filled filter housing with $1 / 8^{\prime \prime}$ tubing connections.

- Hg headspace container: $140 \mathrm{ml}$ glass bottle with a plastic cover, which has the center removed and a rubber septum, mounted. The septum is to allow access for sampling of the headspace with a syringe. 
The headspace sample bottle contains the $\mathrm{Hg}$. The bottle cover has the RDT wired through the septum. The RTD should hang below the cover $1 / 2$ way down the bottle, and not touch the sides of the container. The bottle is then inset into a lead cylinder using thermal paste to aid in making thermal contact between the outside of the glass bottle and the inside of the lead cylinder. The lead cylinder measurements are 4" dia. X 5.5" high with the center bored out to accept the glass bottle. The headspace is vented through the septum using 1/8" Teflon tubing to a charcoal filled filter housing. There is an SRD fabricated headspace assembly that is designed as detailed below.

\section{$\underline{\text { Materials }}$}

These materials are used to support the process of creating and measuring a $\mathrm{Hg}$ gas-phase calibration standard.

- $\quad$ Argon gas, grade 5 (99.999\%).

- $\quad$ Pure liquid mercury, $10 \mathrm{ml}$.

\section{Preparation}

\section{Hg headspace container}

- The complete headspace container assembly is placed into the VWR temperature controlled bath. Water level within the bath should be just below the top of the container assembly. The bath temperature should be set to approximately $0.5^{\circ} \mathrm{C}$ below ambient room temperature. If possible, the bath and headspace assembly should have 24 hours to stabilize whenever the temperature is adjusted.

- Wire the RTD to the multimeter and set it to measure resistance. A temperature of $20^{\circ} \mathrm{C}$ will be measured as approximately $107.77 \mathrm{ohms.}$

\section{$\underline{\text { AFS Setup }}$}

The gas supply system for the AFS is setup to provide $45 \mathrm{sccm}$ of argon gas to the AFS. It runs from the tank regulator to a mass flow controller, a tee fitting with septum, a gold trap, and then into the AFS. Teflon tubing and fittings should be used between the sample injection septum and the AFS. The only exception to this is the glass tubing used to house the gold wire in the $\mathrm{Hg}$ trap. Currently the cylinder source is regulated at $15 \mathrm{psig}$. The mass flow controller is set, using the dipswitches on the back face of the controller, to measure argon. The gas enters the tee fitting through the branch tee connector, which is oriented at $90 \mathrm{deg}$ to the other two connectors on the tee. The remaining two connectors are oriented directly in line with the $\mathrm{Hg}$ gold wire trap. One connector has a septum that will be used to inject the $\mathrm{Hg}$ headspace sample; the other is connected directly to the trap. The trap is located as close to the input fitting of the AFS as possible. The Hg trap has an external Nichrome filament heater, wound in a coil, and located centrally over the gold wire (trap) contained within the glass tube. This heater is wired to the MG DC power supply and set to input 13.5 VDC @ 5 amps.

A schematic diagram of the Brooks Rand Model III AFS along with the various components described above is shown in Fig. 51 below. 
- Turn on the AFS and set the PMT to 500. Allow the lamp to stabilize for some hours prior to taking your first measurement.

- The computer should be running the Brooks Rand Hg Guru software as well as the Excel database.

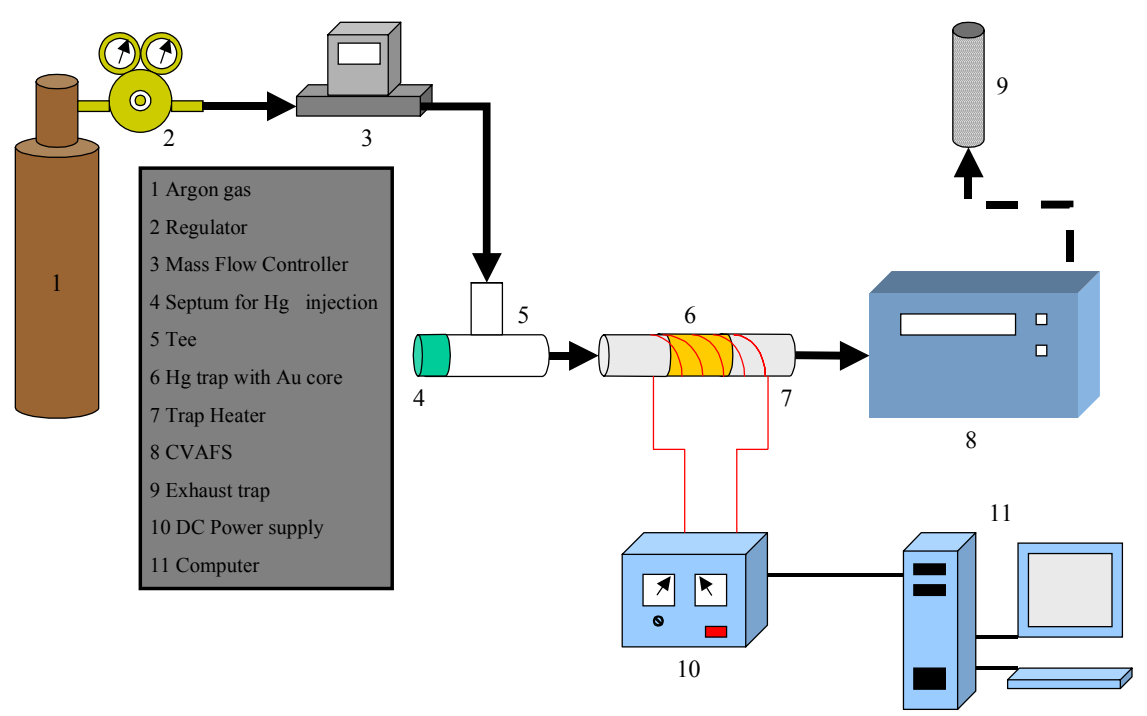

Figure 51: Schematic drawing of the AFS system with the various components listed on the figure.

\section{Syringe}

The syringe should be kept just slightly above room temperature. Precondition the syringe by taking a sample and expelling it back into the headspace. Some prefer a technique where the preconditioning sample is expelled into a filtered fume hood. There are many variations on this sampling technique and it is recommended that a variety of these be tried. Adopt the technique that provides accurate, repeatable, and consistent results. Read the referenced manual and papers to learn these different techniques for headspace sample acquisition. . $^{19,20}$

\section{Procedure}

\section{Preconditioning of the Hg Trap}

Typically the gold in the $\mathrm{Hg}$ trap retains a small amount of $\mathrm{Hg}$ or, over time, it traps some $\mathrm{Hg}$ from the room environment. Consequently prior to administering a headspace sample the trap should be thermally cycled at least once.

- Turn on the argon gas source and the heater power supply. Allow the trap 5 minutes to desorb any incidental $\mathrm{Hg}$ it may have accumulated.

- Turn off the heater and allow the trap to cool for 5 minutes before proceeding further. Leave the argon gas flowing. 


\section{Sample acquisition}

It is recommended that prior to doing this the Brooks Rand manual" and "Tips on making saturated mercury vapor injections ${ }^{\prime 20}$ be reviewed.

The syringe should be stored at a temperature just slightly above the room ambient. Do not handle the body of the syringe anymore than necessary, and when you do handle the syringe, try to minimize the area of contact by holding the syringe using the ears at the plunger end.

- If the syringe plunger has not been pushed all the way down do that first.

- Next, the syringe tip should be pushed through the middle of the $\mathrm{Hg}$ headspace container septum to a depth of approximately $2 / 3$ of the needle length. Do not touch the headspace container bottle with the needle.

- Slowly pull the plunger up at a rate, which will take approximately 20 seconds to fill the syringe to the $2.5 \mathrm{ml}$ level.

- Gently push the plunger back down until the $2.0 \mathrm{ml}$ level is reached.

- Remove the needle from the headspace container and immediately go to the AFS septum injection site.

\section{Sample injection}

- Insert the needle through the septum in the tee all the way up to the glass body of the syringe. Make the injection slowly, taking about 5 seconds to empty the syringe.

- Pull the syringe body back about 1 inch from the tee, but with the tip still inserted through the septum in the tee, and draw in a full syringe of argon gas.

- Push the tip back into the tee up to the glass body (as during the initial injection) and perform a second injection. This is to clear any residual $\mathrm{Hg}$ that may not have been transferred with the first injection. 


\section{$\underline{\text { Sample AFS process }}$}

The headspace sample will have amalgamated with the gold in the trap. However, the system must be purged with argon prior to running any samples, otherwise any oxygen contained in the sample can quench the fluorescence of $\mathrm{Hg}$ leading to erroneous results. With the argon flow rate set to $45 \mathrm{sccm}$ this process will take approximately 5 minutes.

Once the sample, either a headspace measurement or a sample taken from the CRD instrument, is injected into the gold trap the procedure for obtaining the fluorescence data from the AFS is performed as follows:

The timeline used to heat the Nichrome wire around the glass section containing the gold trap is:

0 seconds - start run

30 seconds - heater on

360 seconds - heater off

400 seconds - end run

- Zero the AFS by pushing the Auto Zero button.

- $\quad$ Bring up the Excel database.

- Bring up the Brooks Rand program.

- Set the "Diskwrite interval" to 0.1 seconds.

- Clear the graph area.

- Start the program.

- Create a file name to save the data.

- Click on the file "save" button to start the run, at which point the program starts logging data from the AFS. This is the beginning of the timeline sequence. Follow the process timeline given above.

- Turn off the program to end the run.

$\underline{\text { Data }}$

\section{Equations}

These are the salient equations used in the Excel database.

- Temperature is calculated by taking the resistance measurement of the RTD in the Hg headspace and using,

$$
\mathrm{T} \operatorname{deg} \mathrm{C} .=\left(\left(.001 * \mathrm{R}^{2}\right)+(\mathrm{R} * 2.3599)\right)-245.93
$$

- The number of picograms per microliter of $\mathrm{Hg}$ is calculated by: 


$$
\mathrm{pg} / \mu \mathrm{l}=\left(\left(.0314 * \mathrm{~T} \operatorname{deg} \mathrm{C}^{2}\right)-(.1976 * \mathrm{~T} \operatorname{deg} \mathrm{C})\right)+4.5266
$$

- $\quad$ Total picograms $=$ sample size $* \mathrm{pg} / \mu \mathrm{l}$

- The peak area is calculated by integrating the magnitude, or signal count, of the AFS (sampled at 100 millisecond intervals) over a (user defined) period of 300 seconds. The integrated sum is the peak area.

- $\quad$ Picograms per peak area unit (PA) are calculated by: pg/PA = total picograms / peak area.

- Peak area units per picogram of $\mathrm{Hg}$ is calculated by: $\mathrm{PA} / \mathrm{pg}=$ peak area / total picograms. Average $\mathrm{PA} / \mathrm{pg}$ is substituted for this when $\mathrm{Hg}$ samples from a source other than the headspace are to be quantified. Average PA units/pg are calculated using the mean of the most recent 3 or 4 headspace measurements taken on the same day, but prior to, any other Hg samples.

- The total picograms are using average PA units per picogram is calculated: PA / (PA units/pg)

- Nanograms per minute, $\mathrm{ng} / \mathrm{min},=(($ average $\mathrm{PA} / \mathrm{pg}) / 1000) /$ sample collection time in minutes.

This headspace calibration measurement should be done a minimum of three times, with repeatable results, prior to using the resulting data as a calibration standard for other $\mathrm{Hg}$ measurements.

This completes the headspace calibration process.

\section{Measurements}

Once the headspace calibration measurements are completed measurements can be taken at a number of points throughout the CRD apparatus. Measurements are generally made at the exit of the oven containing the mercury permeation tube to establish the concentration entering the gas sample stream. Measurements are also made at the entrance and exit of the ring-down cavity. This ensures no loss of mercury in the cavity itself or in any of the tubing contained in the CRD apparatus.

Shown in Fig 52 is a plot of the concentration of mercury determined by the AFS versus the CRD instrument. For this plot it was assumed that the AFS results were correct. The blue points are those determined from the CRD instrument. Therefore if the AFS and CRD results agree then the blue points should fall along the diagonal red line. From the plot it can be seen that the CRD data agrees very well with the AFS data except for two points, which appear to be somewhat low. The data depicted on the plot was all the data that has been obtained for the month of March 2004. The data for the low values was taken a number of times and each of the other data points lies along the diagonal. The error could have been caused by measurement error in one or both of the instruments. 


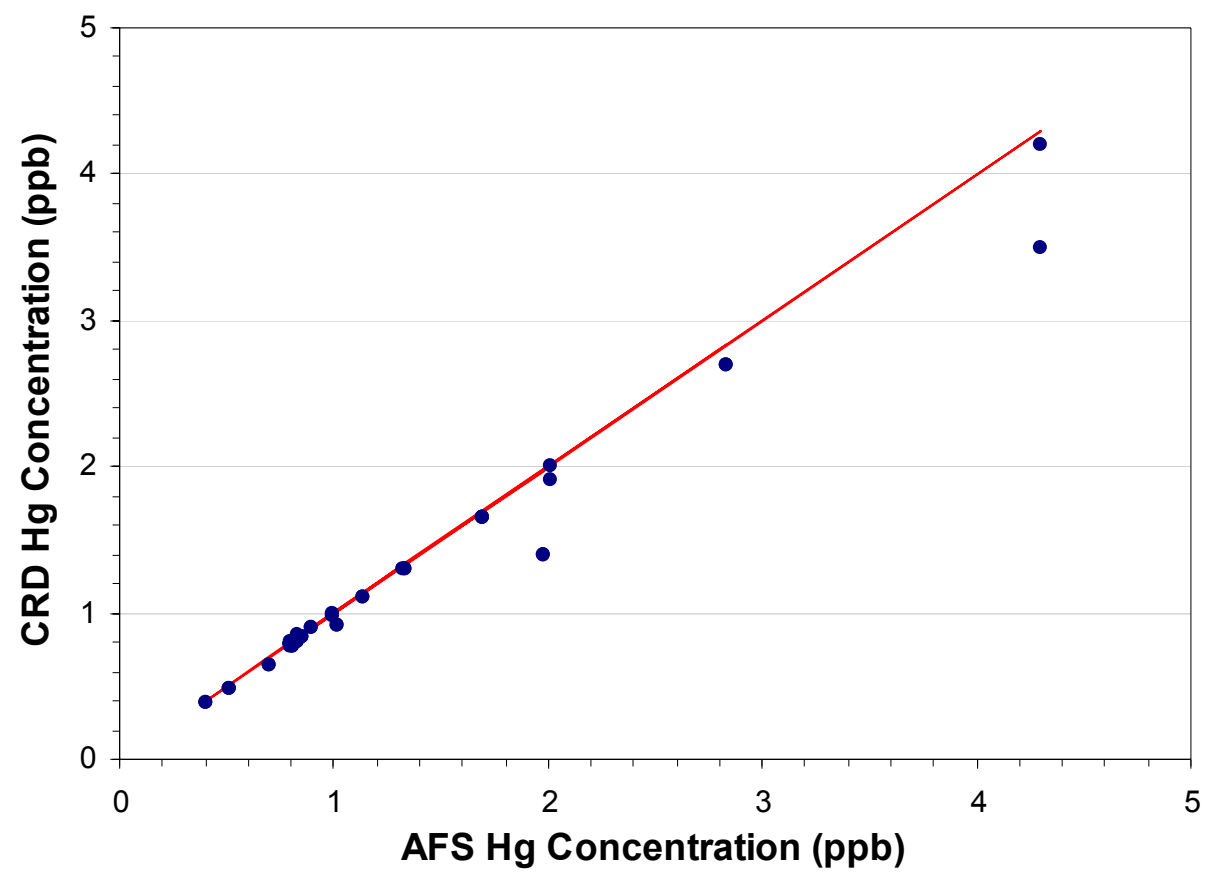

Figure 52: Plot of the mercury concentration determined by the AFS versus that determined by the CRD instrument. The blue points are those determined by the CRD instrument. In the plot it is assumed that the AFS results are correct so is the blue points lie along the red line then the two agree.

$\underline{\text { Safety }}$

Safety equipment that should be on hand for any procedures that involve the use of mercury include:

- A Material Safety Data Sheet (MSDS) for mercury and any mercury containing compounds used.

- A mercury spill control kit should always be on hand. 


\section{Sensor Research \& Development Corporation DE-FC26-01FT41221}

\section{Software Development for CEM Operation}

Software development has occurred on a continuing basis as the cavity ring-down project has progressed. This started with the development of the data acquisition and laser control program. This particular program was used to acquire data by scanning the diode seed laser across the entire mercury transition. The program has been used in the development of the CRD instrument and for a variety of diagnostic tests throughout the entire project. After the initial development the program continued to evolve during the project to respond to challenges as they arose, but always with the goal of developing a CRD instrument capable of performing as a true CEM.

Recent software development for the CRD instrument has focused on developing a program that will run the CRD instrument autonomously as a CEM, reporting mercury concentrations on a continuous basis. This new software development was able to build on the experience gained throughout this project. In addition, many of the subroutines developed to solve previous problems were then incorporated into the new program.

A relatively detailed description of the program and its various functions is given below. First the overall flow of the program will be discussed followed by details of the individual windows available in the program. Also the adjustable parameters will be discussed and their effect on the operation of the CRD instrument.

\section{Overall Program Function}

The subroutines written to correct the problems of drifting and mode hops worked very well. This was shown with the results obtained for the 3 day test, which have been displayed in Figs. 49 and 50. The next task was to incorporate all the changes into a new program that could be used for mercury monitoring, autonomously and on a continuous basis, during a field test. The previous version of the program was used primarily to scan across the mercury transition and perform diagnostics for the various tests done by SRD optical scientists. This was the version of the program that has been used to produce all of the data previously obtained and reported. Shown in Fig. 53 is a simplified flow diagram of the new program developed by SRD software engineers to operate the CRD instrument as a true CEM mercury monitor.

The first thing the program must do to monitor the mercury concentration is determine the total empty cavity losses of the CRD instrument. This is accomplished with a sample flow of clean calibration air through the cavity at approximately the same cavity pressure and temperature that will be used during testing. This baseline loss measurement not only is used to ensure proper operation of the CRD instrument but it is also used in determining both the mercury and $\mathrm{SO}_{2}$ concentrations.

The next step in the process, shown in the flow diagram in Fig. 53, is to find the frequency of the desired mercury peak to be used for continuously monitoring the mercury concentration. The isotopic and hyperfine structure of mercury is such that there are 5 possible peaks that can be used to monitor the mercury concentration (see section on Mercury Detection and Isotopic Structure). Once the desired peak position has been determined the program will determine the concentration level of $\mathrm{SO}_{2}$ present in the sample stream. The program will then enter a loop where it will scan across the desired mercury peak and report the mercury concentration determined. The program will continue to scan across the mercury peak and report the mercury concentration until one of a couple of conditions is met. One such condition is that the program checks the concentration determined with a number of previous determinations, if the value is outside a certain range $(>\mathrm{x} \%)$ the program will jump out of the loop and return to find the desired 
mercury peak again. This condition will occur during a mode hop where a drastic frequency change will result in a drastic reported mercury concentration change. This change, or other anomalous event, will trigger the program to reacquire the correct mercury peak, re-determine the SO2 concentration, and continue reporting accurate mercury concentrations. The other condition that will cause the program to exit the mercury concentration determination loop is an automatic save. While the data file is continually updated with the reported concentration data, there is a settable time period where the program will close out one file and begin a new one. When this is done the program again returns to find the desired mercury peak, determine the $\mathrm{SO}_{2}$ concentration, and continue with the mercury concentration determination loop.

The program will only end when the user clicks on a button within the program ('Press to Stop Sequence') at which point the program will close out the last data files.

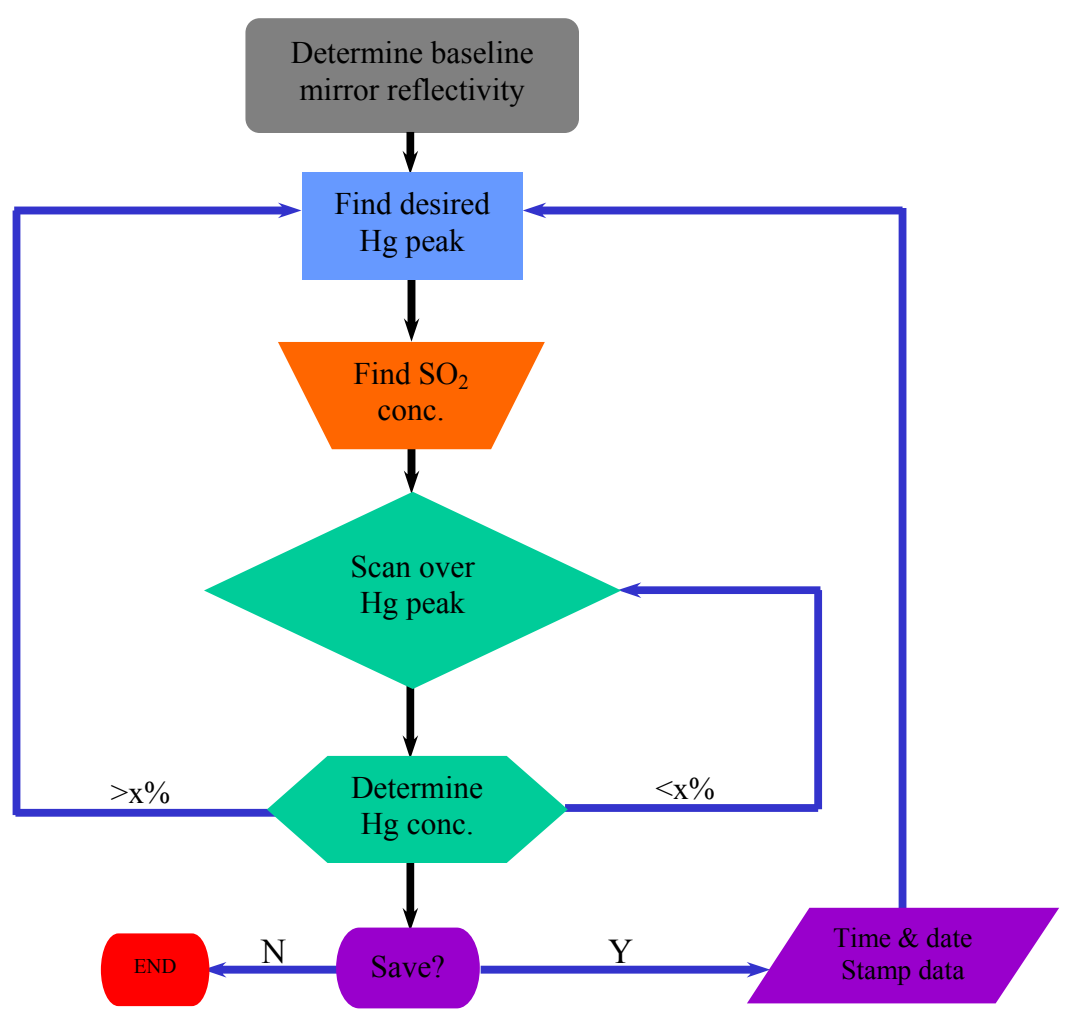

Figure 53: Simplified flow diagram of the new program used to operate the CRD instrument as a continuous emissions monitor. The program is setup to obtain and report mercury concentration, on a continuous basis, as well as report the concentration of sulfur dioxide.

The process described above is summarized in the flow chart shown in Fig. 53. This is just a short outline of the new program's overall function. The new program has been designed by SRD software engineers to include a number of different windows that can be viewed, at any time when the program is running, to observe the mercury concentrations or to adjust any number of parameters that affect the operational characteristics of the program. Shown in Fig. 54 is an example view of the front panel of the program, which will appear when the program has been initiated. Near the top left corner of the panel a number of tabs can be seen. These tabs are labeled: Front; Main; X-Peak; Other Controls/Indicators; and Oscope. These tabs can be used to navigate between the various windows to access the variety of different functions available to the program user. 


\section{Sensor Research \& Development Corporation DE-FC26-01FT41221}

\section{Front Page Window}

An example of the Front page of the program is shown in Fig. 54. The light gray portion of the window is the area of the window associated with each particular tab. Along the right side of the full program window is a darker gray column that does not change when different tab windows are being viewed. This continuous display area is divided into three sections. The top button ('Power On') within the top section is to turn the program on and initialize the various subroutines in the program once the program has been initially started. The next button down ('Press for Single Capture') initiates a single ring-down measurement at a set particular voltage using the various parameters set for number of pulses averaged, particular gates to use for the single fit, etc. The button immediately below this ('Press to Start/Stop Sequence') is used to initiate mercury concentration measurements once all of the adjustable parameters have been set.

The final button of this group ('Press to find true $R$ ') is used to determine the baseline reflectivity of the CRD cavity with only clean air contained in the cavity. This is the first step shown in the flow diagram given in Fig. 53. To perform this task there must be a flow of clean air through the CRD cavity. It would be best if the pressure and temperature of the cavity were maintained at, or close to, the conditions that will be used during actual testing. While not necessary, this will ensure a proper mirror alignment that could possibly change with either large pressure or temperature changes within the cavity. Once this task has been completed the program will store the value of the losses due to the mirrors and use this for subsequent calculations of both $\mathrm{SO}_{2}$ and mercury concentrations. This task can be performed at any time that the program is running if the user feels that the baseline losses may have changed or wishes to verify that they have remained constant. When the button is clicked the program will re-determine the baseline losses due to the transmission of the mirrors and store this new value.

The next group down the right side, labeled as 'Sweep Parameters', contains a number of parameters that are constantly updated as the program is determining mercury concentrations or performing additional functions. The top parameter in this group ('Current Voltage') gives the value of the voltage presently being applied to the diode seed laser. The next parameter down ('Current Step \#') indicates the step number within the particular function the program is performing at that time. The parameter ('\# of seq run') indicates the number of times a certain function has been performed, such as how many times the program has scanned across a certain mercury peak to determine the concentration. The green 'Seq?' indicator beside this parameter indicates if the program is performing a sequence function and will be dark if the program is performing a one-time function, such as a single ring-down capture event. Below these sequence indicators is a parameter ('new center') that gives the voltage value, applied to the diode seed laser that is associated with the determined central point of the mercury peak chosen to monitor the concentration. This value is re-determined after each scan across the mercury peak to correct for any drift in the frequency output of the diode laser. The final parameter in this group ('peak') is the last total cavity losses value determined from scanning across the desired mercury peak. This is the value that is used to calculate the mercury concentration.

The last group of parameters listed down the right side of the screen ('File Parameters') contains parameters associated with storing the data files. The first parameter listed ('experiment time') keeps a record of how long a particular sequence, or experiment, has been running. This is a continuously running time that will only reset once the 'Press to Stop Sequence' button has been clicked by the user. The next parameter, 'New file every', is adjustable, even while the program is performing a set function, with both an hour (' $h$ ') and minute (' $m$ ') setting. This setting indicates the period of time the program will update a particular data file before it is closed and another file is started. This is the point at the bottom of the flow chart shown in Fig. 53 where the data file is closed and the program returns to find the desired mercury peak to continue determining the concentration. 
The last two parameters ('Full Sweep' and 'Peak Sweep') are the file names that are used for the automatic saves performed by the program, using the 'New file every' settings mentioned above. The full path and file name are listed in the two boxes. Once the first file has been saved the program automatically increments the file names by adding a sequential number to the end of the file names used. The 'Full Sweep' file is used to store data obtained when the program performs the function to find the desired mercury peak that will be used to monitor the mercury concentration. The second file, 'Peak Sweep', is used to store the data obtained by the program when scanning across the desired mercury peak. This is the file that also contains the information about both the mercury and sulfur dioxide concentrations determined by the CRD instrument.

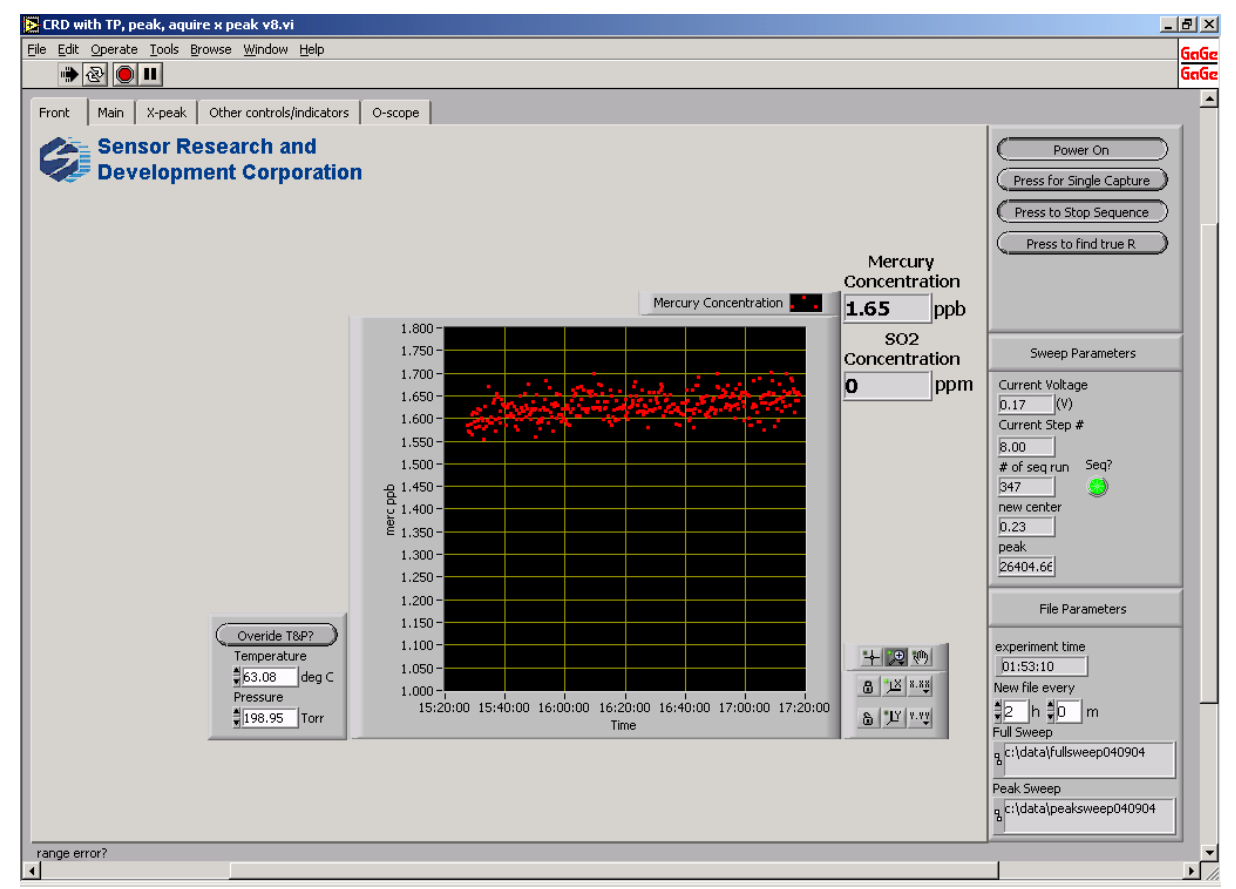

Figure 54: The Front display panel of the program used to operate the CRD instrument as a CEM. The tabs shown at the top left corner are the different displays viewable while the program is running and where a variety of parameters can be adjusted. Along the right side of the window is a display of various parameters that remain regardless of which tab is being viewed.

The Front panel display shown in Fig. 54 contains a single plot that displays the continuously obtained mercury concentration (red dots). The plot is given as time, in minutes, versus mercury concentration in ppb. In addition on the upper right side of the plot the most recently obtained mercury concentration and $\mathrm{SO}_{2}$ concentration are reported in boxes as numbers with the mercury concentration given in $\mathrm{ppb}$ and the $\mathrm{SO}_{2}$ concentration given in $\mathrm{ppm}$. Near the bottom left of the plot is a display, which gives a continuous readout of the temperature and pressure readings inside the CRD cavity. The small box just to the right of the bottom right corner of the plot are a number of settings that can be used to change the values of the axes, the display settings for the data, and tools to zoom or move the plot around. 


\section{Sensor Research \& Development Corporation DE-FC26-01FT41221}

\section{Main Window}

An example of the Main window of the program is shown below in Fig. 55. This is the window that displays the actual ring-down curves, fits to the averaged curves, and the resulting voltage scans, of the diode seed laser, across the desired mercury peak to be used to monitor the mercury concentration.

The smaller plot visible in the middle on the left hand side of the window is a plot of the output of the 100 $\mathrm{MHz} \mathrm{A} / \mathrm{D}$ card. This plot displays the results of each individual laser shot. The horizontal axis on the plot is time units, which are dependent on the data acquisition rate set for the card. This value is generally 100 $\mathrm{MHz}$ unless a second channel of data is used which would drop the rate to $50 \mathrm{MHz}$. The vertical axis in the plot is individual bits from the 12-bit card. The value of each bit is determined by the voltage scale settings for the A/D card. The larger plot located directly above it, in the upper left corner of the window, is a display of the averaged ring-down curve. This is an average of a certain number of laser shots as chosen by the user. The horizontal axis on this plot is time where the units are in microseconds. The vertical axis is the actual voltage value obtained from the photomultiplier tube. There is a small button to the upper right of the plot labeled 'invert?'. The actual data obtained is a negative going voltage, which has been inverted for fitting and display purposes. This option allows the inverting function to be turned ' $O F F$ ' or ' $O N$ ' depending on the specific application.

Also visible on this plot are two red vertical lines going from the top to the bottom of the plot area. These are the boundaries that are used to define the area of the curve to be fit. There are two separate methods for choosing the position of these boundaries. The first is by manually setting the position of the two gates on the ring-down curve. As the program is triggered by each individual ring-down event the change in timing position for subsequent averaged ring-down curves is minimal. However, as the laser scans across a mercury transition, or if sulfur dioxide is present in the gas stream, the ring-down time can change dramatically. If the gates were set for a long ring-down time the fit for a very short ring-down time would fit mostly the flat tail of the curve rather than the important decay at the start of the curve. As a result the second option for fitting the averaged ring-down curve is to use percentage gates. When using this option the program determines the maximum intensity of the ring-down curve and calculates the percentage values and sets the appropriate positions for the two gates. The program does this for each of the averaged ring-down decays obtained and thereby focuses the fit on the important part of the decay curve. There are also two settable gates between which the program obtains the baseline voltage level, which is subtracted from the ring-down decay for the final fit. These two gates are set manually. The value for these two gates given in Fig. 55 is prior to the start of the display area of the graph, which has been set to observe just the ring-down decay, and is hence not visible on the display in the figure.

Shown to the right of the averaged ring-down plot are the parameters that are used to determine both the baseline and ring-down decay gates that are used in the fitting routine. The box on top of these parameters has a button labeled 'Percent Gates'. This button can be used to switch between the manually set gates and the percentage set gates. Below this button are the 'start' and 'end' percentage settings used to determine the gate positions when using this percentage gate mode. In the example shown in Fig. 55 the first gate is set to $80.0 \%$ of the maximum intensity of the ring-down curve while the second gate is set to $10.0 \%$ of this maximum value. The actual voltage values used for the various gates are given in the box directly below the percentage gate settings. They are labeled 'Baselinel', 'Baseline2', 'Datal', and 'Data2'. The value given just to the right of each of these parameters is the time position of each gate in microseconds, relative to the trigger position. In the example shown in the figure the average baseline value would be determined between -2.00 and $-0.50 \mu \mathrm{sec}$. The fit to the exponential decay of the ringdown curve would occur between 0.03 and $0.18 \mu$ sec. 
The plot visible in the lower left corner of the window are the results obtained from fitting the averaged plot, show in the upper left corner. The fit shown in the example in Fig. 55 is a linear fit. The horizontal axis displays the number of points that are used in the fit, which are separated in time depending on the speed of the data acquisition card. In this example the points are 10 nanoseconds apart as the card is running at a sample rate of $100 \mathrm{MHz}$. The vertical axis is the natural logarithm of the voltage value, and hence why the fit is linear. The white points in the plot are the actual data while the red line is the fit line.

A number of values obtained from the fit are listed along the right side of the fit plot. The first value listed, 'loss in ppm', is the total measured cavity losses per pass, in parts-per-million, obtained from the fit to the averaged ring-down decay curve. The next value listed, 'Tau', is the ring-down time obtained from the slope of the linear fit in microseconds. The last three values listed are associated with the errors determined from the fit. The first of these, 'mse', is the mean square error determined from the fit. This value is the sum of the squares of the residuals divided by the number of points fit. The next value, 'dloss', is the error determined for the total losses in the cavity in ppm per pass. The last value in the list, ' $d$ Tau', is the error associated with the determination of the ring-down time, given in microseconds.

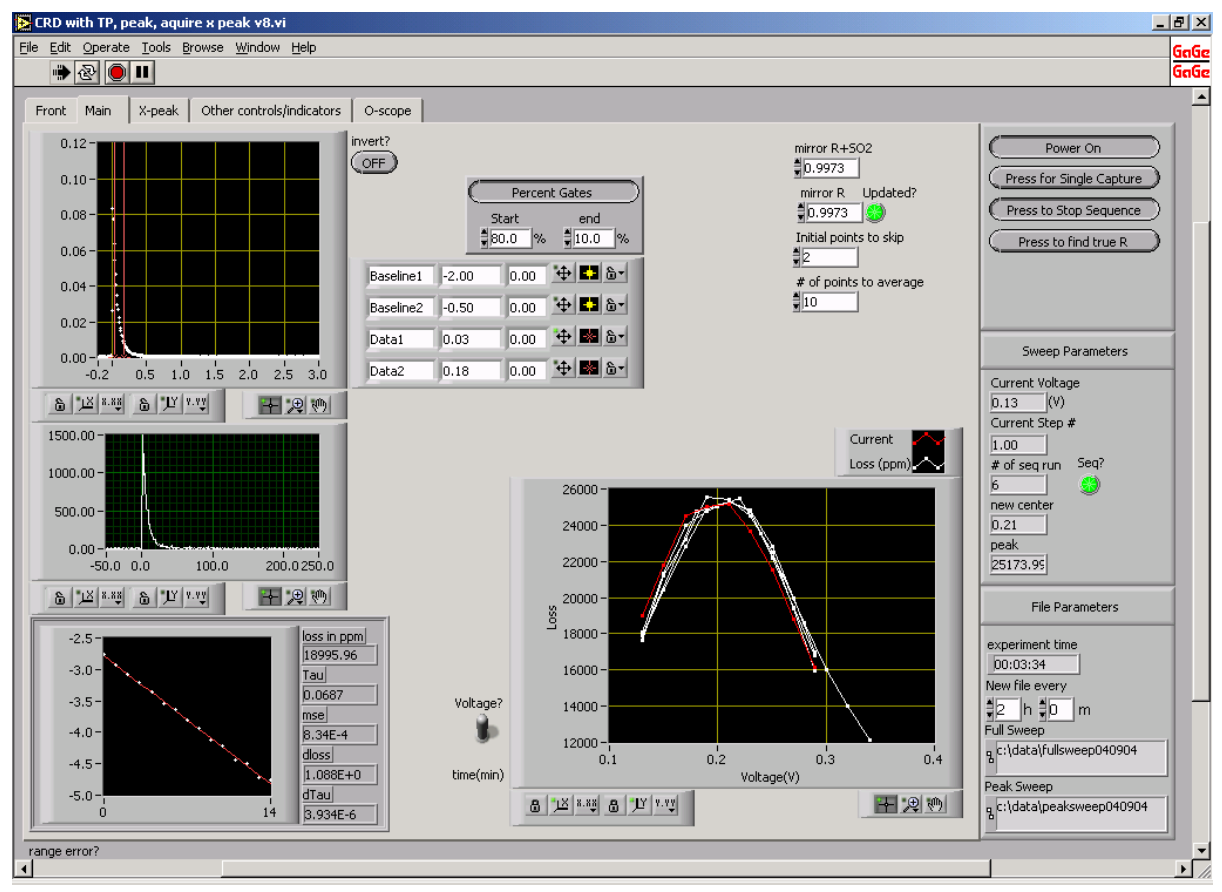

Figure 55: An example of the display observed on the Main page of the program. There are a number of plots displaying a variety of data used to determine the mercury concentration (see text).

The last plot shown on the Main page can be seen in the bottom right corner in Fig. 55. This is the plot used for displaying data taken as the program scans back and forth across the desired mercury peak. There is a toggle switch just to the left of the plot, which will change the plot from total cavity losses versus time ('time ( $\mathrm{min})$ ') to total cavity losses versus voltage ('Voltage?') applied to the diode seed laser. In the example shown in the figure the display is set to losses versus voltage applied to the diode laser. The display will retain all of the previous scans across the desired mercury peak with the presently scanning line displayed in red. This allows the user to visually check that the laser is indeed scanning across a mercury peak and to see if the center voltage is drifting over time. The two small boxes located just below this plot are parameters to adjust the axes of the plot, how the particular data points are displayed, or adjustments to zoom or move the plot around depending on the users preferences. 
Other than the various plots described above there are only 4 remaining values displayed on the Main page. These can be seen in the upper right corner of the window shown in Fig. 55. The second value shown, 'mirror $R$ ', is the reflectivity of the mirrors determined from the ring-down time observed for a cavity with a clean sample of air. This is the initial function displayed in the flow chart shown in Fig. 53. This value is determined when the button, 'Press to find true $R$ ', just to the right of this value is clicked. In addition to the displayed value there is a reminder light to indicate if this function has been performed. This green light is located just to the right of the reflectivity value under the heading 'Updated?'. This light will remain dark after the program is first initialized until the mirror reflectivity determination function has been performed. Once this task has been performed the indicator will turn bright for the duration of the particular sequence being run.

The value located just above this baseline mirror reflectivity value, labeled 'mirror $R+S O 2$ ', is used to determine the sulfur dioxide concentration. When the program searches for the desired mercury peak it automatically checks the baseline total cavity losses off resonance from any mercury peaks. The program then compares this value with that obtained for the cavity with clean calibration air. If there are additional losses detected in the sample stream background the program assumes that these losses are due to $\mathrm{SO}_{2}$. This assumption has been made as tests performed by SRD scientists to all other components of flue gas showed no, or insignificant, absorption in the same spectral region as mercury. The program takes the difference in losses between the baseline losses and the additional losses and calculates an $\mathrm{SO}_{2}$ concentration. This concentration is entered into the data files as well as displayed on the Front page of the program. This function is performed each time the program re-determines the frequency position of the mercury peak that will be used to monitor the mercury concentration. As an example, after each automatic save the program would measure the $\mathrm{SO}_{2}$ concentration before proceeding to monitor the mercury concentration.

The final two values displayed, in the top right corner, are used by the program in determining the baseline value of the CRD instrument. This function is performed every time the program scans for the desired mercury peak. The first of these values, labeled 'Initial points to skip', are the number of points from the very start of the scan to omit from the calculation of the baseline value. This is done to ensure that the losses in the cavity are truly at the baseline level as a large change in frequency can result in a slight error in the ring-down decay as the laser 'locks' to the new frequency. The next value, labeled '\# of points to average', are the number of consecutive points, after the omitted ones, which are used to calculate the average value for the baseline losses of the CRD instrument. 


\title{
Sensor Research \& Development Corporation DE-FC26-01FT41221
}

\author{
X-Peak Window
}

The next tab viewable in the program is labeled $\mathbf{X}$-peak. This is the window where the particular mercury peak that will be used to monitor the mercury concentration is chosen. There are also a number of parameters adjustable when scanning for the desired mercury peak as well as a number of settable parameters, which are used when the program is monitoring the mercury concentration, to determine if the program should rescan to find the desired mercury peak. This function is used for correcting problems associated with mode hops or if the diode seed laser frequency drifts off of the mercury peak despite the constant re-centering.

The box located in the upper left corner of the window has a button at the top of the box labeled 'Press to find $x$-peak'. Clicking this button will cause the program to reacquire the desired mercury peak regardless of the function the program is performing at the time. There are 6 different parameters listed below this button to set a number of conditions the program then uses to determine when it actually finds a mercury peak.

The first parameter, 'Find Peak \#', is a setting that can range in value from 1 to 5 . This will be the mercury peak that the program will use to monitor the mercury concentration. The numbering is from low frequency to high frequency. Peak number two, the 202 mercury isotopic peak, is the largest peak and the one most used for monitoring the mercury concentration. An example of the 5 different isotopic peaks can be seen schematically and graphically in Figs. 8 and 9 respectively, and the natural isotopic percentage for each of the mercury isotopes is given in Table 1 .

The two values listed at the bottom of this small box in the upper left corner are values that are used to determine a threshold value that the program only looks above in determining the mercury peak positions. The parameter listed on the bottom left, 'threshold as \% of baseline', is the percentage above the baseline level determined in the scan that the program sets as the threshold. In the example shown in Fig. 56 this value is set to $125 \%$ of the baseline value. As the program initiates the scan to find the desired mercury peak there are a number of points off resonance at the low frequency end of the scan. The program ignores the first $x$ points and then uses then next $y$ points that are averaged to determine the baseline value. The parameters $x$ and $y$ are adjustable within the Main window. The parameter on the bottom right of this box, 'min threshold for peak', is the calculated loss value, from the baseline losses and the input percentage, used as the threshold value by the program. This threshold value was added to the program as it was found that the program would occasionally determine baseline noise spikes as mercury transitions.

The three remaining parameters, in the 'find $x$-peak' box, are utilized by the program in determining the peak position of the desired mercury peak. The program determines specific peak positions by using a window of a certain number of points. Within this window the program takes a certain number of points at each end of the window. The program then checks for a peak, which is above a particular preset value, in between the ends of the window. If the program determines that these conditions are met it will assign a mercury peak to the maximum value contained within the set window. The parameter listed in the middle of the left side of this box, "window size', is the number of voltage steps that are used for the window to check for a peak. The parameter to the right of this value, 'end size', is the number of points at each end of the window whose values are used to determine if there is a mercury peak in between the two ends of the window. The program will calculate an average value of the end points when determining if a peak is observed. The last parameter used to determine the mercury peak positions, 'multiplier', is listed on the top right of this box. This multiplier is applied to points in between the two ends of the window. If the total observed losses at any point within the window, multiplied by the 'multiplier' value, is greater 
than or equal to the average value at both ends of the window the program will determine this point to be a mercury peak.

In the example shown in Fig. 56 the window size has been set to 10 points and the ends of the window have been set to 2 points. The mercury peak chosen is the ${ }^{202} \mathrm{Hg}$ single isotopic peak (peak \# 2). The threshold value has been set to $125 \%$ of the baseline value, which is calculated as $3327 \mathrm{ppm}$ total cavity losses in this example. The multiplier has been set to 0.990 , so any value within the window that is $1 \%$ or greater above both ends of the window will be counted as a mercury peak.

The results of the settings can be seen in the only graph shown in this window. The plot is voltage applied to the diode seed laser versus total cavity losses. The thick horizontal yellow line depicts the calculated threshold value that the program is using for this particular scan. This value is listed again at the bottom right of the plot as 'Threshold' and the value is given. The red line shown below the yellow line is the actual data obtained from the CRD instrument as the laser is scanned. Also visible in the plot is a white box, which is located on top of the two mercury peaks. The program will label the points determined as a mercury peak with these white boxes. In addition to the red line indicating the observed data is a thicker yellow-green line that is plotted on top of the red data line. This line is the window within which the peak positions are determined. In real-time the line will travel along the plot and the program will mark mercury peaks as the program scans across them. Once the program has reached the desired mercury peak, in this case the second peak, it will exit from this function and begin scanning across the desired mercury peak and report mercury concentrations.

There is one box containing a number of parameters on the X-peak window. This is located near the center-top of the window and labeled as 'For auto find $x$-peak'. These are the parameters that the program uses while determining mercury concentrations to decide if the concentration value obtained might be erroneous as would occur if a mode hop were to happen or if for some reason the diode seed laser frequency drifted off of the desired mercury peak.

In essence, the program will take a number of previously obtained concentration values and determine if the next value fits within a certain criteria. If the value does fit within the specified criteria the program continues determining the mercury concentration. If the value is outside of the specified criteria the program will exit out of the mercury concentration determination function and reacquire the correct mercury peak to ensure that the proper concentration is being reported. 


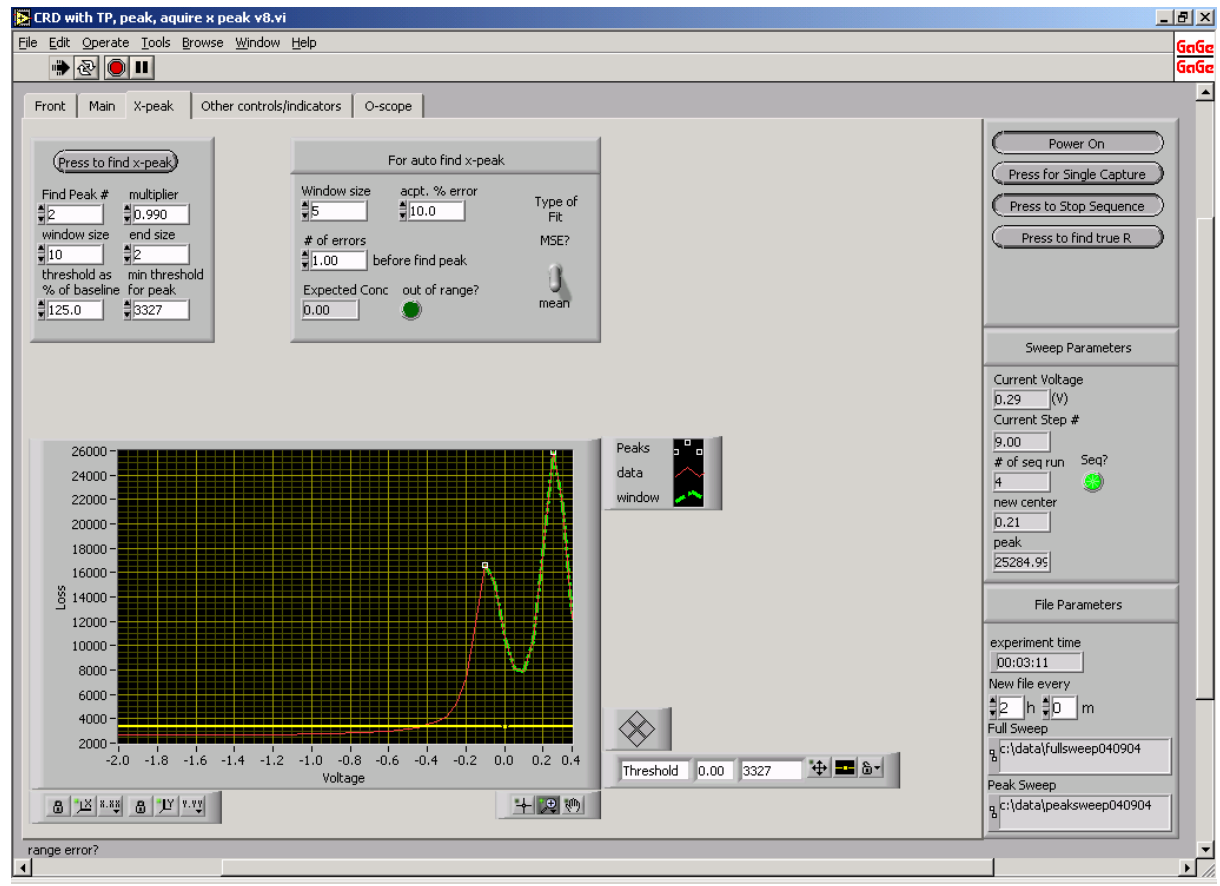

Figure 56: The display shown for the X-Peak tab. There are a number of adjustable parameters viewable in the two boxes in the upper left corner of the display (see text). In this example the second mercury peak has been chosen as the monitoring peak for reporting the mercury concentrations.

The parameter found in the upper left portion of this box, 'Window size', is the number of previously determined mercury concentration values that the program uses to compare the next point with. The parameter box located just to the right of this value is labeled 'acpt. \% error'. This parameter is the acceptable percentage error, compared with the previous $x$-points, which the next value must be within. The parameter located in the center left of this box is labeled as '\# of errors', with 'before find peak' written just to the right of the value for this parameter. This value is the number of mercury concentration points that are outside of the acceptable error parameter before the program will exit out of the concentration determination function and switch to reacquire the desired mercury peak. The last value listed in this box is labeled 'Expected Conc'. This is the expected mercury concentration that was determined from the last time that the program acquired the desired mercury peak. The indicator light, just to the right of this value, is labeled 'out of range?'. This indicator light will turn on when a determined mercury concentration is outside of the acceptable parameters that have been set within the 'For auto find $x$-peak' box.

There is a single toggle switch located within the 'For auto find $x$-peak' box, labeled 'Type of fit' with the two options 'MSE?' and 'mean'. This toggle switch determines the type of fit to compare the next mercury concentration data point with the previous $x$-points. The 'mean' option determines the average of the previous $x$-points to compare to the next point. The 'MSE?' fit is a least squares fit of a line to the previous $x$-points. The former of these two options is best used when the mercury concentration is relatively constant while the latter option is best used when fluctuating mercury concentrations are expected.

In the example shown in Fig. 56 the number of points, used to compare the next data point to, are the previous five concentration values. The acceptable error, for the next point, from the previous 5 points is 


\section{Sensor Research \& Development Corporation DE-FC26-01FT41221}

$10 \%$ and the type of fit used is an average of the 5 points. The number of errors before the program exits the mercury concentration determination mode has been set to one. This means that the first time the program detects a mercury concentration point $\pm 10 \%$ away from the average of the previous five points it will re-determine the position of the desired mercury peak (in this case the second peak) to ensure that the reported mercury concentration is accurate.

\section{Other Controls/Indicators Page}

Shown in Fig. 57 is the window labeled Other Controls/Indicators. This window contains a couple of parameters that are used by the program for determining the mercury and sulfur dioxide concentration, but the majority of the window is used to acquire data from a second data channel available with the 100 $\mathrm{MHz}$ A/D card.

The parameters related to the determination of the mercury concentration are given in the bottom left corner of the window. The toggle switch located above these parameters is labeled as 'Type of fit'. The fit that this switch refers to is the way that the actual averaged ring-down decay is fit. There are two choices: 1) 'Linear', and 2) 'Exponential'. As the decay curve is itself an exponential decay, using the latter of these two options directly fits the curve to an exponential decay. The former of the two fits takes the natural logarithm of the voltage intensity and fits it to a line. In the example shown in Fig. 57 the fit type is set to 'Linear'. This is also the type of fit that was displayed in the example given in Fig. 55 of the Main page of the program.

The parameter listed directly below the type of fit is the 'cavity length $(\mathrm{cm})$ '. This parameter is for the sample pathlength through the cavity, given in centimeters. In most cases the cavity length and sample pathlength are the same but it is possible for these two distances to be different. The sample pathlength is important, as it is one of the parameters used in Beer's law to calculate the concentration from the measured absorption in the cavity. This parameter is a user input.

The two values listed below the cavity length are two parameters related to the data acquisition card. The first, 'Actual SR', is the actual sample rate being used by the card. Generally this value is $100 \mathrm{MHz}$, the maximum rate of the card, but when two channels are used to acquire data this value will be cut in half for each channel. The second parameter, 'Trigger Level', indicates the voltage level, in volts, of the trigger signal being used to trigger the data acquisition card, which can be the ring-down signal itself or an external trigger source.

There are two graphs displayed on this page. The first, located in the upper half of the page, displays the data obtained from the second channel of the A/D card. The data displayed in this graph is the averaged data obtained from the specified number of laser shots. The axes for this graph are: voltage, in volts, along the vertical axis; and time, in microseconds, along the horizontal axis. Located adjacent to this plot, near the bottom left corner, are a listing of four parameters. The four parameters listed are: 'Baselinel', 'Baseline2', 'Datal', and 'Data2'. These parameters define the gates that are used to obtain data from the input signal. Their function is similar to those described for the averaged ring-down decay plot from the Main page. The two baseline gates define the points between which the baseline value used in calculations is taken. The points between these two gates are averaged to obtain the baseline voltage value. The two data gates define the region used for the data calculation. This data channel has been set up to integrate the area of signal between the two data gates and subtract off the obtained baseline value. As an example, this data channel has been used for monitoring the intensity level of the fundamental 
radiation from the Alexandrite laser system, monitored with a photodiode, as well as the absorption of the fundamental beam through an iodine cell for absolute wavelength calibration of the laser system.

The graph shown on the lower portion of the page is labeled 'Intensity as a function of Voltage $(V)$ '. The integrated intensity, obtained from the graph shown above, is plotted versus the voltage applied to the diode seed laser. The small boxes near the bottom right corner of this plot are parameters to adjust the axes of the plot, the way the data is displayed, or to zoom to different portions of the graph.

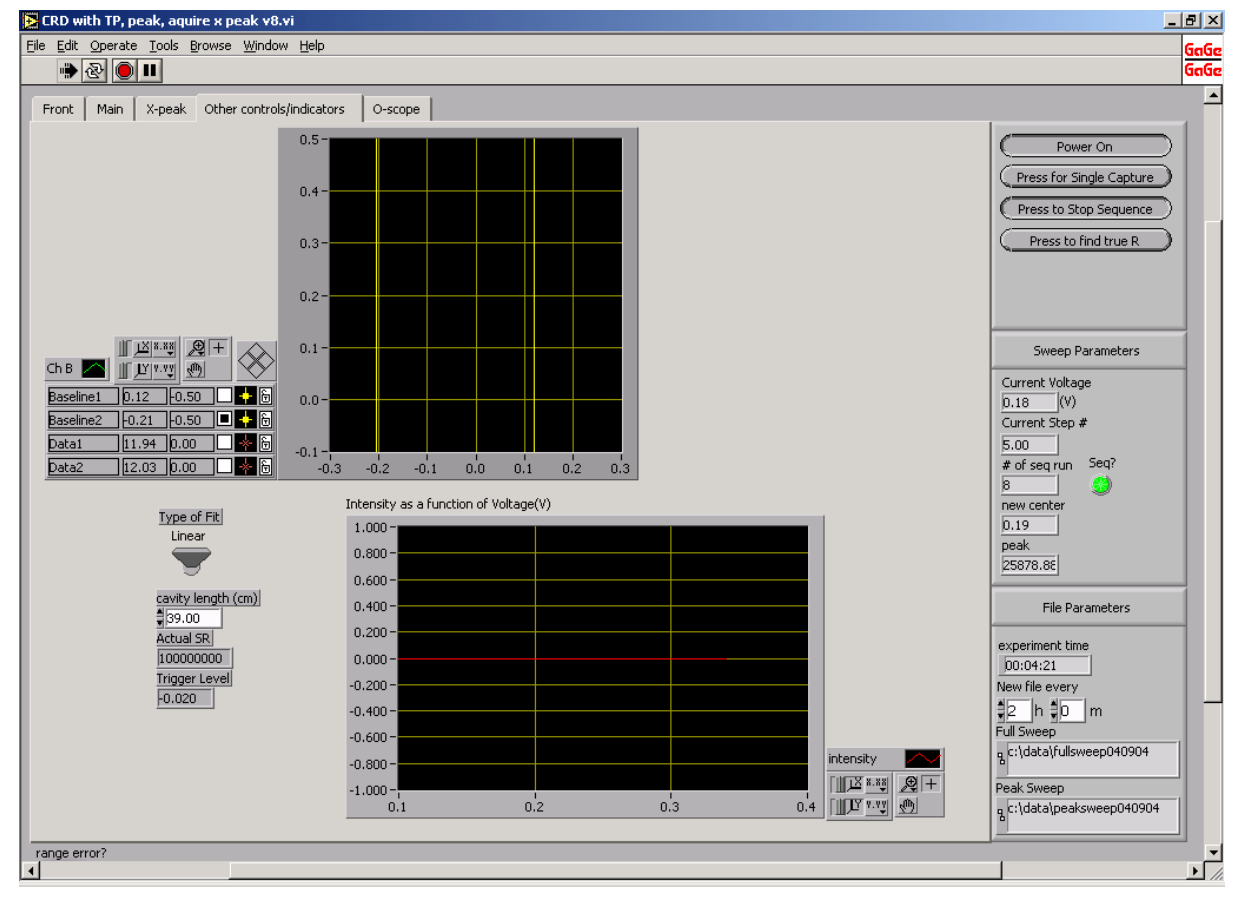

Figure 57: An example of the Other Controls/Indicators page. This page, while not presently used, has been used in the past for data input into a second channel on the A/D converter.

\section{O-Scope Controls Page}

The final page of the program that may be accessed from the tabs on top of the window is the O-scope page. An example display of this page can be seen in Fig. 58. The main function of this page is the settings associated with the digitizing card that is used to acquire the ring-down data. There are also two boxes with parameters related other functions of the program. One is for the function of finding the desired mercury peak and the other is related to the function of scanning across the desired mercury peak to obtain the correct mercury concentration.

The large box extending down the left hand side of this page contains the settings for the digitizing card and are typical of those used with most oscilloscopes. The A/D card used to obtain data for the CRD instrument is a 12-bit card with a $100 \mathrm{MHz}$ sampling rate, from GaGe Applied, Inc. (Model CS12100). The top left section within this box contains the settings for the two available data channels on the card (' $C h A$ ' and ' $C h B$ '). The top two switches listed under ' $C h A$ ' and ' $C h B$ ' is for whether the input voltage is either ' $A C$ ' or ' $D C$ '. The input from the CRD instrument is always a DC voltage. Directly below the voltage switch for each channel is the full-scale voltage setting for both channels. A sliding indicator can 


\section{Sensor Research \& Development Corporation DE-FC26-01FT41221}

be moved from $100 \mathrm{mV}$ to $5 \mathrm{~V}$ with 6 different settings for each channel under the heading ' $C h A R$ ' and ' $C h B R$ '. The indicator sets the voltage scale used by the program as $\pm \mathrm{x}$ volts, where $\mathrm{x}$ is the setting chosen. The 12-bit voltage resolution from the digitizer is then split equally between the + and - voltage scales.

The next set of parameters, listed below the channel settings, involves the trigger settings for the digitizing card. The top parameter listed, 'Trig Level', refers to the sliding scale along the right hand side of the trigger source parameters box. The scale goes from -128 to 127. The blue arrow, shown along the scale, can be moved up or down to any value in between the two limits. The actual voltage value of the trigger from this setting is given on the Other controls/indicators page.

Below the trigger level is the parameter labeled 'Trig Source'. There are four options for the source of the trigger to initiate data acquisition by the digitizing card, they are: 'Software', 'External', 'Channel B', and 'Channel $A$ '. The software trigger is one that can be set internally to the program to run at a certain frequency. The external trigger will work from a TTL, or similar, type pulse input into the card. The last two are for triggering off of the data signal directly. Generally the CRD instrument is triggered from the ring-down signal directly. This will eliminate any slight shifts in time of the averaged ring-down decay curve relative to static gates that are set up to calculate the ring-down time and hence the mercury concentration.

The next parameter listed among this group is labeled 'Trig Slope'. There are only two options for this parameter: 'Negative' and 'Positive'. This switch will determine if the digitizing card looks for the trigger pulse from a negative going or positive going voltage. The switch below the slope parameter is labeled 'Trig Coupling'. The options for this switch are: ' $A C$ ' and ' $D C$ '. This parameter is used to set whether the trigger signal used is an AC or DC voltage. Located below this coupling parameter is one labeled 'Trig Range'. The options available for this parameter are either ' $1 V$ ' or ' $5 V$ '. This parameter sets the voltage scale to be used for the trigger level explained above. If the ' $1 \mathrm{~V}$ ' setting is chosen that means that the 128 to 127 scale corresponds to \pm 1 Volt, and likewise \pm 5 Volts for the ' $5 \mathrm{~V}$ ' setting.

The final parameter listed in the trigger parameters box is labeled 'Points'. This is the total number of points available, both pre- and post-trigger on the digitizing card. In the example shown in Fig. 58 this value is set to 8192 , which means there are 4096 points available pre-trigger and 4096 points available post-trigger. The ultimate value of this number is determined by the amount of memory available on the particular digitizing card used. The CRD instrument has never used a total of 8192 points so this value has been set as the default value for this parameter.

At the top of the page located next to the Channel parameters are two parameters labeled 'Capture Mode' and 'Sample Rate'. The first parameter has two options: 'Single Chan.' and 'Dual Chan.'. This is the parameter that is set to take data either from just Channel A or from both Channel A and Channel B. The sample rate given below this parameter is the chosen sampling rate of the digitizing card. The maximum rate for the presently used $\mathrm{A} / \mathrm{D}$ card is $100 \mathrm{MHz}$, which is the speed that is always used by the CRD instrument. If however the 'Dual Chan.' option is chosen the actual sampling rate will be half of the value chosen in this parameter box. The actual sampling rate, at which the digitizing card is running, is given under the Other controls/indicators page.

Directly below the capture mode and sample rate is a small box with 3 different parameters. The first two are labeled 'Start' and 'Number'. These two parameters are used to indicate the actual number of points to use from the total number available, given as 8192 above. The 'Start' parameter indicates where, from the trigger position, the program should start to acquire data. The 'Number' parameter indicates the total number of points, from the 'Start', to obtain for the data. In the example shown in Fig. 58 the actual data 
points obtained by the program would begin at 250 points before the trigger and contain a total of the next 1024 points. At the maximum $100 \mathrm{MHz}$ sampling rate of the $\mathrm{A} / \mathrm{D}$ card the time period for the 1024 points used would correspond to $10.24 \mu \mathrm{sec}$. This is more than enough time, as the ring-down time of the empty cavity is less than $1 \mu \mathrm{sec}$ for the $38 \mathrm{~cm}$ cavity that is used for the CRD instrument.

The last of the three parameters listed in this box is labeled 'Times'. This is the setting for the actual number of laser shots to average for each data point. This parameter can be set to any positive integer value. From Fig. 58 it can be seen that this parameter is set to 50 . That would mean that 50 laser shots would be averaged and then the ring-down time would be calculated for that averaged waveform. This is the plot that is displayed in the upper left corner of the Main page.

The final parameter listed in the digitizing card parameter box is labeled 'Impedance'. This is the value of the input impedance of the digitizing card. There are two options for this setting. The first is $50 \mathrm{Ohm}$ and the second is $1 \mathrm{MOhm}$. The ring-down decays occur on very short time scales and as a result the $50 \mathrm{Ohm}$ setting is used.

Located at the top center of the page is a box labeled 'Find $x$-Peak or single capture'. This box contains a number of parameters that are used when the program scans to ascertain the frequency position of the desired mercury peak to be used for monitoring, or when the 'Press for Single Capture' button, located with the settings to the right of the page area in the program window, is clicked.

The first parameter listed in this box is labeled 'Start Voltage'. This is the initial voltage that is applied to the diode seed laser. This voltage is generally set to a value that corresponds to a frequency well below that of any mercury resonant transition. This is to ensure that the scan begins with a number of flat baseline points to establish the baseline value that is used in determining the baseline loss level of the $\mathrm{CRD}$ instrument, the $\mathrm{SO}_{2}$ concentration, and the mercury concentration.

The single capture function of the program is often used for diagnostic purposes. When the 'Press for Single Capture' button is pressed the program will move the laser position to the 'Start Voltage' setting and acquire the number of laser shots that are set to be averaged for each point and calculates the total losses within the cavity at that single voltage setting. This is a useful function when sitting on either the baseline or a mercury peak and tweaking the CRD instrument. The voltage limits for the piezoelectric tuning element of the diode seed laser (Newport Corporation, Model 2010M) that is being used with the Alexandrite PAL laser is \pm 7 Volts. This voltage range corresponds to a frequency range of approximately $85 \mathrm{GHz}$ at the fundamental wavelength of $761 \mathrm{~nm}$, or about $255 \mathrm{GHz}$ in the UV around the mercury transition. The entire width of the mercury transition is only about $30-50 \mathrm{GHz}$, depending on cell pressure, which is less than $20 \%$ of the diode laser's full tuning range. 


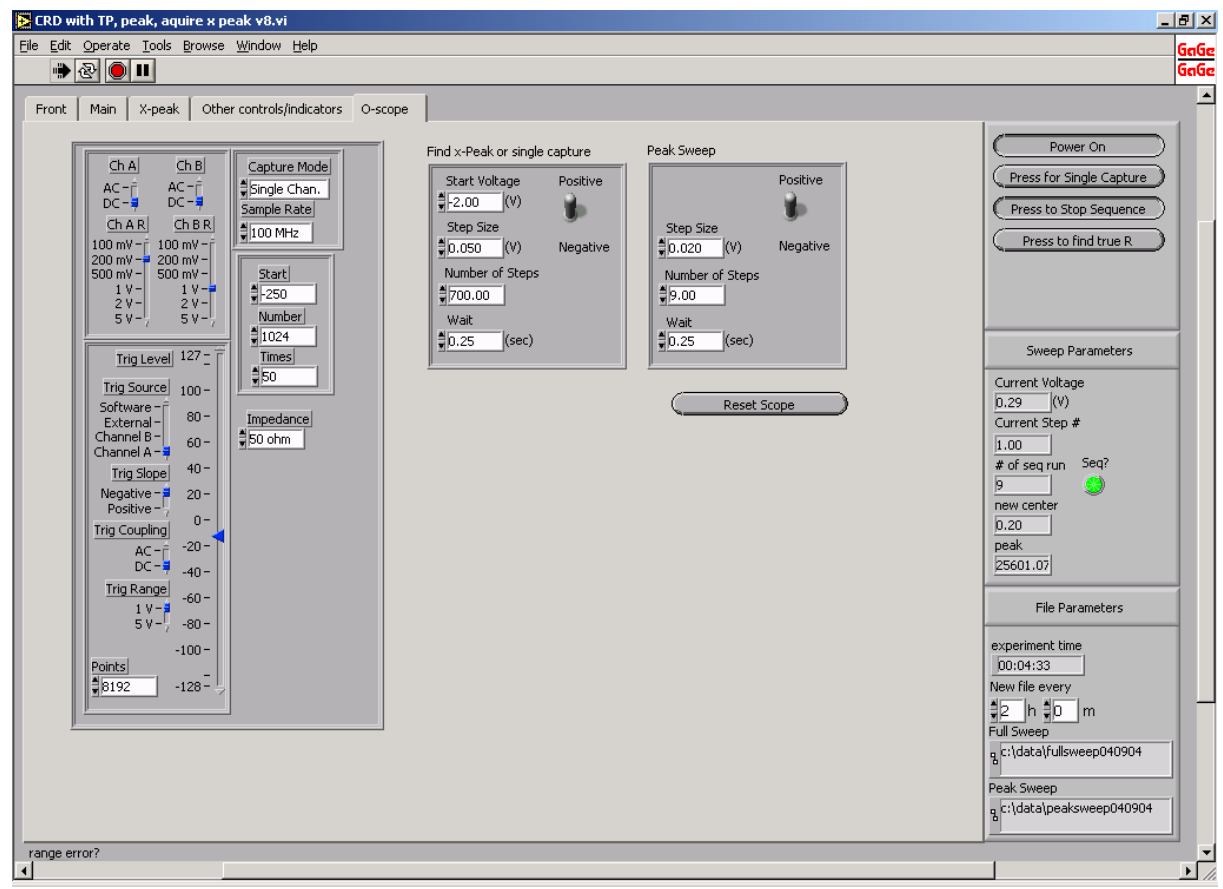

Figure 58: The display observed for the O-scope page. This page contains all of the settings for the fast A/D card used to obtain the ring-down data. Also there are parameters that can be adjusted for the step size and number of steps taken for both the function that first finds the desired mercury peak, and for the function that scans back and forth across the desired mercury peak to obtain the mercury concentrations.

Below the starting voltage is a parameter labeled 'Step Size'. This is the voltage step size that is used when scanning over the mercury transition to obtain the frequency of the desired mercury peak that will be used to monitor the mercury concentration. The next parameter down in this box is the total number of steps for the program to take during a scan to find the desired mercury peak. The program will either take the input number of steps or go to the voltage limit, whichever it reaches first. At this point if the program has not found the desired mercury transition it will report a mercury concentration of $0 \mathrm{ppb}$ and rescan the region again. It will repeat this process until mercury is present and the desired mercury peak has been found.

The parameter below the number of steps is labeled 'Wait'. This parameter is the amount of time, in seconds, the program waits after each step before it begins taking data. Once the frequency of the diode seed laser is changed it takes the Alexandrite laser system a short amount of time before it is 'locked' to the new frequency, hence the first few shots of data may not accurately portray the real data at that frequency point. In the example shown in Fig. 58 this parameter has been set to 0.25 seconds, which corresponds to 12 laser shots, as the system runs at $50 \mathrm{~Hz}$. This is more than enough time for the laser to 'lock' to the new frequency without significantly effecting the time required to obtain each individual mercury concentration point.

The last parameter visible in this box is a switch labeled 'Positive' and 'Negative'. This is for the direction of the voltage scan relative to the start voltage and step size. A positive setting would scan the voltage by adding the step size to the start voltage, while the negative setting would subtract the step size 


\section{Sensor Research \& Development Corporation DE-FC26-01FT41221}

voltage from the start voltage for each step. As a result the diode seed laser can be tuned either by increasing or decreasing the frequency.

There is a box just to the right of the box described above labeled 'Peak Sweep'. There are a number of parameters listed in this box that are identical to the 'Find $x$-Peak or single capture' box. These parameters have the same function as the identical parameters described above. The parameters listed are: 'Step Size', 'Number of Steps', and 'Wait'. There is also a 'Positive' and 'Negative' switch. The difference between these two boxes is that the peak sweep parameters are used by the program when scanning back and forth across the desired mercury transition to obtain the mercury concentration, while the find x-peak parameters are used to located the desired mercury transition. From the example shown in Fig. 58 it can be seen that the program is set to use 9 steps with a step size of 0.020 volts to obtain the mercury concentration.

From the example given in Fig. 58 the differences in setting between the two boxes, 'Find $x$-Peak or single capture' and 'Peak Sweep' can be noted. The step size is significantly larger when doing a crude scan to find the desired mercury peak ( 0.05 volts $)$ compared to that used when scanning across the mercury peak to determine the mercury concentration $(0.020$ volts $)$ where it is much more important to find the actual peak height. Also the number of steps used to find the desired mercury peak is quite large (700) while that used to determine the mercury concentration is fairly small (9). The value chosen for the 'Peak Sweep' number of steps is important, as it will change the time needed by the CRD instrument to determine each mercury concentration data point. This value is generally kept to the minimum number of points needed to determine the mercury peak height value. The pressure within the CRD cavity can also affect this setting. As the pressure is increased and the pressure broadening becomes significant a greater number of points are needed to ensure that the program is able to determine the mercury peak height. Similarly is the pressure is lower a smaller number of points can be used. For the settings shown in Fig. 58 , and the Alexandrite laser running at $50 \mathrm{~Hz}$, it can be seen that the time needed to obtain each individual mercury concentration data point is about 10 seconds.

The last parameter listed on the O-scope page is a button labeled 'Reset Scope'. When pressed this button will clear all the data from the digitizing card and reinitialize the scope. This concludes the description of the pages available with the program and the parameters available on each individual page.

\section{Pilot Field Test}

\section{Background}

The pilot-scale test took place at the DOE/NETL $500 \mathrm{lb} / \mathrm{hr}$ coal-fired combust or facility in Pittsburgh, Pennsylvania. The test was arranged to piggyback on one of a series of on-going 5 day DOE/ NETL mercury experiments investigating the mercury flue gas emissions from coal furnaces. The running time of the combustor was approximately $10 \mathrm{hr} /$ day during which coal was burned. As visiting contractors, access to the site is limited to the period between 6 A.M. and 6 P.M. For a number of reasons the test, tentatively scheduled for August/September, slipped until October 2004.

\section{Initial Setup (September 22 -24)}

Due to tentative test date and potential schedule conflicts, it was decided to set up the CRDS system prior to the setting of the actual test date. In association with a technician from Light Age, Inc., the alexandrite laser was set up and realigned. This realigned procedure, although successfully completed, took longer than initially planned limiting the time available for system evaluation. 


\section{Sensor Research \& Development Corporation DE-FC26-01FT41221}

It was also discovered at this point that the covers for the laser and cavity were incorrectly constructed. Insufficient time existed to address the problem. The covers were adjusted to meet safety concerns; that is, ensure there were no exposed laser beams. However, the covers could not be used as intended with respect to assisting with temperature control of the system.

\section{Test (October 3-8)}

On Sunday, October 3, we began initialization procedures. This involved turning on the equipment to allow for thermal stabilization. The cavity and sample line temperatures were set slightly above that of the flue gas to avoid any condensation problems. The final assembly of the system, and the mounting of various optical components was performed.

To ensure the CRDS system was warmed up and thermally stable before the test was begun, we started up the laser at approximately 6:30 A.M. This schedule should have provided ample time for the system to reach operational condition and provide ample opportunity for final integration and initial testing before the actual NETL test began. However, once the laser was switched on, it very quickly became apparent that the laser alignment had changed since the original set up and alignment on September 23.

A program to realign the laser was begun immediately. While that was underway, the final integration of system components was completed.

The laser alignment was improved but the resulting delay meant that it had not been possible to optimize the CRDS system. That is, neither a stable ringdown signal from the CRDS cavity nor the measurement of the mirror reflectivity that are needed to ensure that the program was performing as expected had been made, it was decided to proceed with the original test plan. Although the alexandrite ring laser requires careful alignment, provided the environment is reasonably thermally stable, once aligned the laser requires minimal adjustments to remain aligned more or less indefinitely.

On arriving to the laboratory prior to the start of test run, it was found that the laboratory in which the laser was located was so cold that it took the laser almost 3 hours to reach its operating temperature and was seriously out of alignment. It became evident at that time that the building had no thermostatic temperature control. Due to an apparent miscommunication, it had been assumed that the laboratory was temperature controlled to the level of a typical office building. Coupling this with the problem with the covers and the sensitivity of the laser alignment to temperature, the laser fluctuations arising from the thermal misalignment of the laser became the major obstacle to completing the test plan and thus the ability to complete an evaluation of the technique.

On October $6^{\text {th }}$, the system was tested and operational. The laser was marginal (at best) but the system was successfully yielding ringdown measurements. On October $7^{\text {th }}$ It was discovered that both the laser and the cavity had moved out of alignment again overnight. This was attributed to temperature variations in the facility as the outside temperature decrease d into the 30's F overnight. The cavity was quickly realigned. The laser alignment was tweaked but its performance remained marginal at best. The performance of the laser was such that the laser output would range from zero to normal from pulse to pulse.

One of the advantages of cavity ringdown spectroscopy is its insensitivity to fluctuations in the laser power. However, it is necessary for the signal to be sufficient to allow for an exponential decay to occur in the cavity. Although the software is designed to eliminate instances when the laser did not pulse, it was not able to compensate when the laser pulse power was such that the ringdown decay was no longer 
single exponential. Under the conditions prevailing, the laser fluctuations were such as to give rise to a large increase in noise in the calculated ringdown signal.

The options available were:

i) to continue with the test plan with the laser as is or

ii) attempt a complete realignment of the laser.

It was estimated that, at best, a full re-alignment would take at least a day, possibly two full days. Since in the worse case the DOE/NETL test would be over before the alignment was completed, it was decided to proceed with the experiments with the laser operating as it was.

However, before proceeding to the planned experiments, an additional experiment was added. This experiment measured the $\mathrm{Hg}$ concentration directly from the Dynacalibrator permeation tube source into the CRDS cavity in an attempt to estimate the impact of the laser fluctuations on system.

As is evident in Figure 59, this experiment demonstrated that there was a significant increase in fluctuations in the measured $\mathrm{Hg}$ concentration. The test parameters were similar as those used to obtain the data in laboratory, but instead of a very stable signal, the standard deviation in the data had increased by over a factor of five times.

Although it was apparent that these fluctuations would also be present in any stack measurement, it was decided to proceed to sample flue stack gas. Initial measurements did not demonstrate $\mathrm{Hg}$ detection. As expected, the fluctuation in signal (noted in Fig. 59) carried over into the sample measurements resulting significant larger background noise (Fig. 60) than seen in the laboratory (Fig. 61). In fact, the increase in noise was such that the system could not settle on a mercury peak and it was evident that even part per billion concentrations, easily detected under normal operating conditions, would be buried in the background noise. Note: The technique has the proven sensitivity to measure mercury down to the ppt level. In addition, it must be noted that this test was performed when the NETL test was evaluating techniques to remove as much $\mathrm{Hg}$ in the exhaust gas as possible. The absence of any knowledge of the actual concentration of mercury present in the gas stream greatly complicated our attempt to optimize the system under these conditions. 


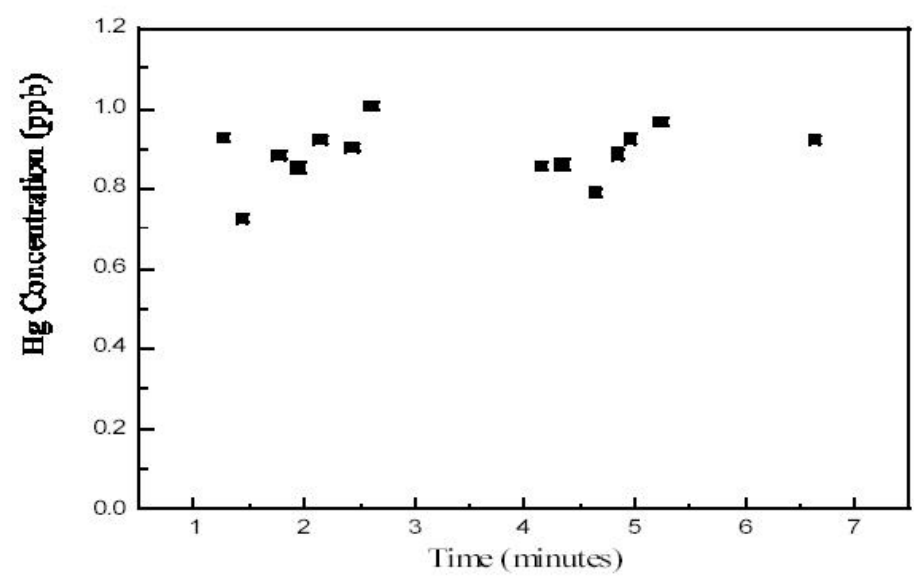

Figure 59: Fluctuations in mercury concentration due to laser misalignment.

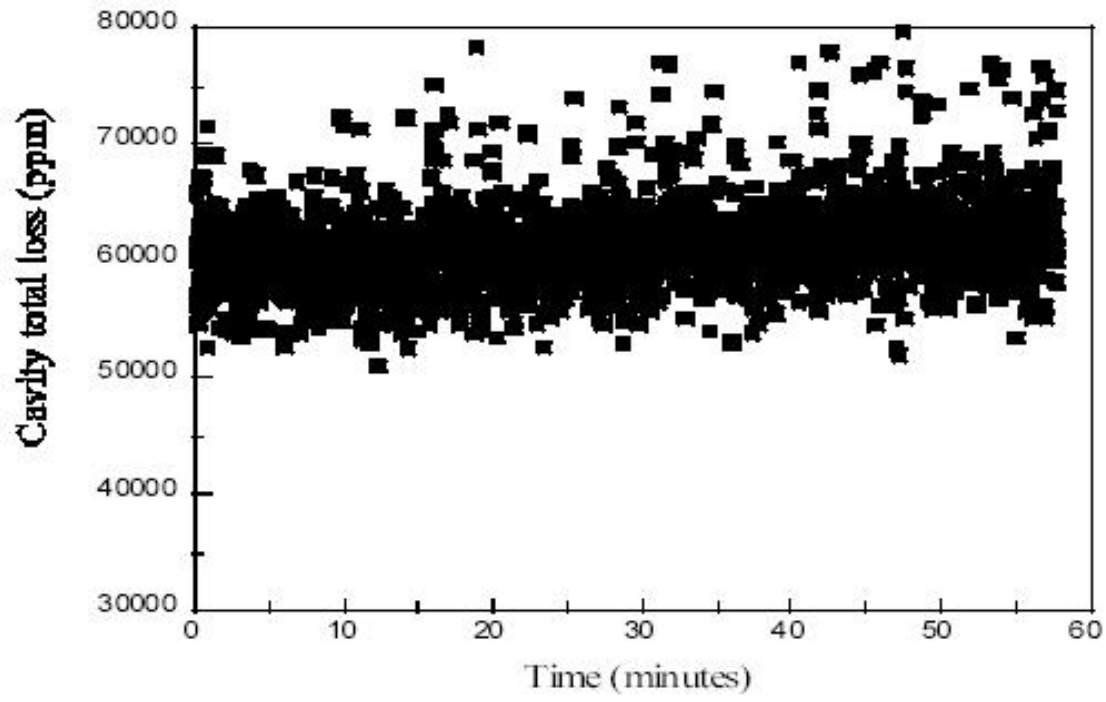

Figure 60: Cavity ringdown measurements of the exhaust gas. 


\section{Sensor Research \& Development Corporation DE-FC26-01FT41221}

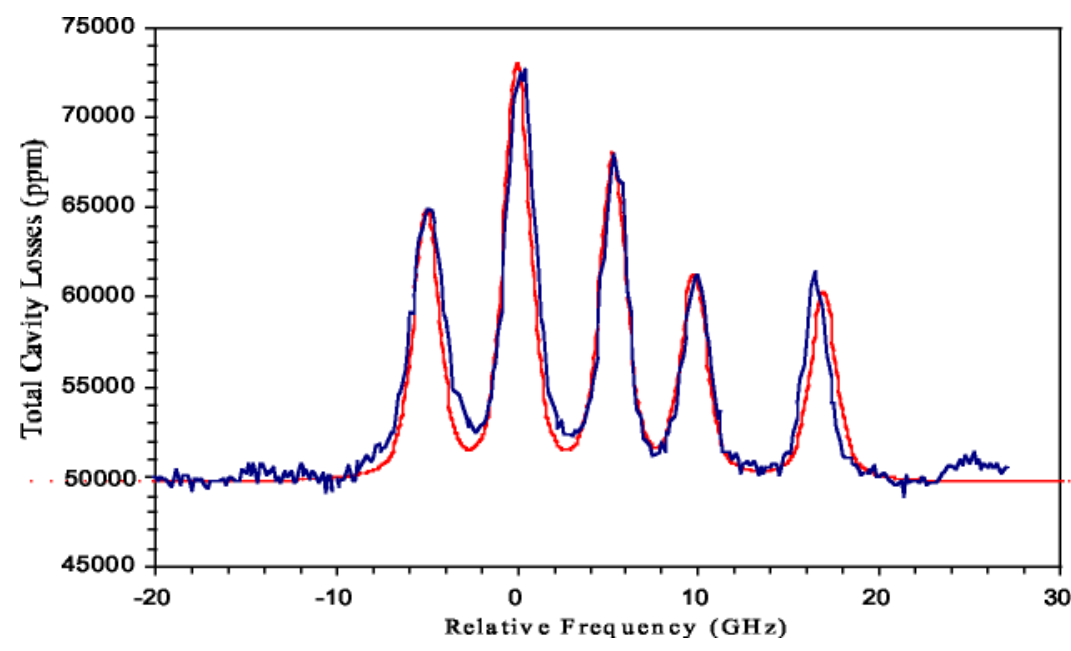

Figure 61: Cavity ringdown measurements under optimum laser operation. $\mathrm{T}=200^{\circ} \mathrm{C}, \mathrm{P}=110$ torr, $1.8 \mathrm{ppb} \mathrm{Hg}, 2000 \mathrm{ppm} \mathrm{SO} 2$

The results presented in Figure 60 confirmed that the noise level is such as to be sufficient to bury any mercury signal, at the levels to be expected in the exhaust gas. However the average level of loss is within the expected range and there does not appear to be any unexpected interferences present in the exhaust gas. Also, although the laser was operating far from optimum condition, thereby drastically reducing sensitivity, it was still proved possible to run the system unattended for several hours.

To provide further confirmation that the problem was due to the laser and not arising from some other cause, such as pressure fluctuations or an unidentified component in the exhaust gas, the pressure inside the cavity was started at 100 torr and increased up to approximately 500 torr. Lowering the cavity pressure will decrease the losses within the cavity thereby allowing an assessment of the operation of the instrument at all different pressure conditions. The results indicated that the noise level was dominated by the laser instability and not from any other cause.

Due to the low outside temperatures, the facility turned on the heating system. Under these circumstances the rise in temperature in the laboratory was such that the laser temperature controller could not control the system temperature and the laser system's temperature increased from its normal 36 $\operatorname{deg} \mathrm{C}$ to almost $40 \mathrm{deg} \mathrm{C}$, with a concomitant decrease in laser performance.

Sample Line Test: Losses between the stack and CEM are, and will likely remain, a critical issue in development and application of continuous emission monitors. As mentioned earlier, it was necessary to borrow a standard $15 \mathrm{ft}$ heated sample line from the NETL facility to allow the CRDS system to obtain a sample. This resulted in a makeshift interface between the exhaust stack and the CRDS furnace. Therefore, an attempt was made to determine whether there were any mercury losses in the sample interface. To measure the loss, an attempt to spike the exhaust gases being withdrawn from the stack with the mercury output from the dynacalibrator was made.

The dynacalibrator output was introduced at the interface of the exhaust stack and sample line and again at the end of the sample line just prior to the system's furnace, As evidenced by Figures 62 and 63, the increase in scatter in the mercury measurements was very large. Nevertheless, it is estimated that the system was experiencing losses of between $25-50 \%$ over the length of the sample line. This apparent loss reflects the need to optimize the CEM's sampling interface to avoid such problems. 


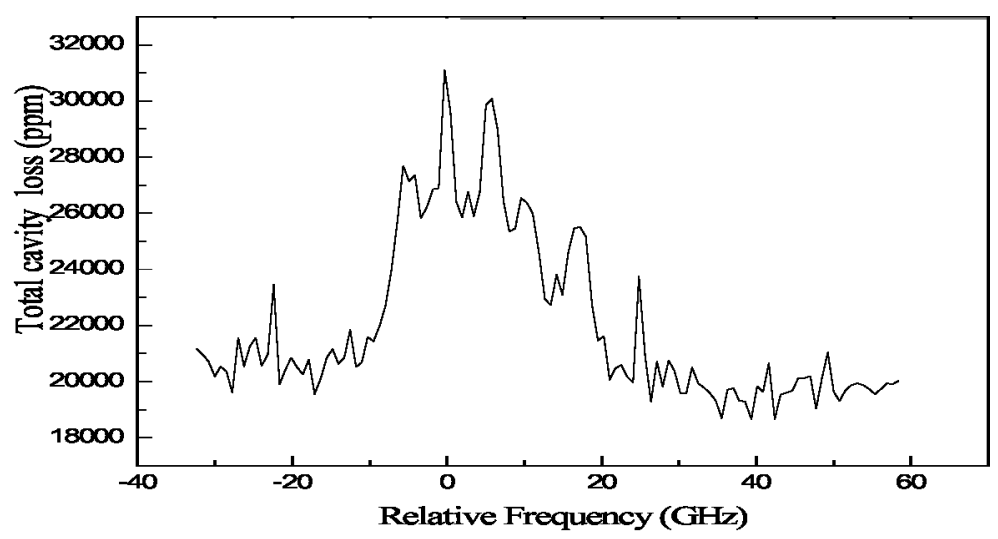

Figure 62: Mercury spectra profile over $253.7 \mathrm{~nm}$ region at 400 Torr. Ran dynacalibrator output into the furnace w/flue gas.

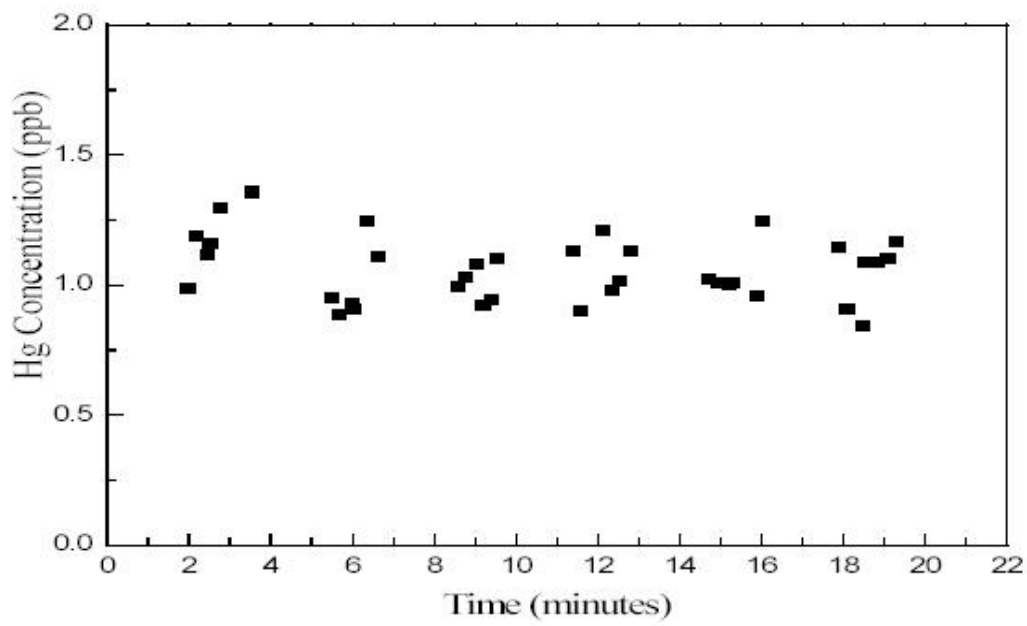

Figure 63: Plot of the concentration of $\mathrm{Hg}$ versus time. Combined dynacalibrator output with flue gas. Cavity pressure 400 Torr.

Spike Test: As indicated above, the DOE/NETL test plan involved testing $\mathrm{Hg}$ absorbance methods (on October $7^{\text {th }}$ ) resulting in very low to zero levels of $\mathrm{Hg}$ in the flue gas. In an attempt to circumvent both the extremely low levels of mercury present and the higher laser noise, a request to use mercury spiked sample line (used by DOE -NETL to carry elemental mercury to their CEMs), was made and approved. This line contained exhaust gases mixed with a known and controllable mercury concentration.

The pressure of the NETL spiked line was 30-1b/square inch. Unfortunately, a heated flow controller to control the gas flow and step down the pressure was not available. In an attempt to jury-rig an inline and the cavity, a surge of pressure resulted in the loss of signal. After checking the system's lack of response using the Dynacalibrator, it became apparent that there had been a substantial reduction in reflectivity of the CRDS cavity mirrors. It appears that the surge in pressure had either blown some material from the furnace into the cavity, and that had coated the mirrors, or had the pressure surge caused a major misalignment. Attempts to realign the cavity did not solve the problem. To investigate the problem further required that the cavity be cooled back to room temperature before disassembly. 


\section{Sensor Research \& Development Corporation DE-FC26-01FT41221}

This event occurred at approximately 5 P.M.; however, all contractor personnel had to leave the facility before 6 P.M. This prevented the researchers from prompt necessary repairs plus reheating of the cavity before vacating the facility. An examination of the mirrors did not indicate any obvious physical damage. The heating to the cavity was turned off to allow it to cool off.

On October 8, which was the last day of the NETL test, it was determined that because of the time necessary to fix the previous day's problem, the test was effectively over. That is, there was insufficient time remaining to make repairs and bring the system back up to a stable operating temperature before the conclusion of the facility test run. Therefore it was decided to archive the data for later analysis. The system was disassembled and packed to await the decision regarding a second test.

\section{Field Test Conclusions}

The instability of the output of the laser prevented the research team from performing a full evaluation of the technology. However, although the test did not meet all of its goals, it did eliminate concerns regarding a number of potential problems:

i) The experimental evidence indicates that no unexpected interferences were present in the coal exhaust.

ii) No problems arose from the presences of particulates. During the testing (prior to the problem with the spike line) the mirror reflectivity was repeatedly checked and no drop-off was observed until the incident with the spiked gas. This indicates that, under normal operating conditions, the exhaust gases will not negatively impact mirror reflectivity.

iii) Water condensation on the windows or other 'cool' areas in the sampling system did not appear to be a problem. However, as iv) indicates further testing is recommended in this area.

iv) CRDS experiments did indicate a drop in $\mathrm{Hg}$ concentration across the heated sample line. Sample line losses are a major concern for any mercury CEM and the fact that we encountered this problem is not unexpected under the circumstances. Substantial work in needed in this area with respect to this instrument.

As mentioned above, the major concern centers on the laser and its requirement for thermal stability. The lack of suitable covers compounded the problem but the test conditions were found to be outside the range at which the laser can be operated stably. One temporary solution would be to provide a temperature-controlled environment for the laser as well as a redesigned container for the CRDS cavity. The long -term solution, as envisioned at the beginning of this project, is the replacement of the alexandrite laser with a more robust light source.

\section{Field Test Recommendations}

Cavity ringdown spectroscopy is an inherently simple technique that has the sensitivity and selectivity to provide the foundation of a continuous emission mercury monitor. Its potential to extract sub-ppb level mercury concentrations on a real-time basis in complex gas mixtures present in coal exhaust gases is unique. The SRD effort leading up to this field test reconfirmed the underlying science. However, considerably more research and development work is required to bring this technology up to the standard required to meet the stringent real-world demands expected of mercury CEM.

To meet such requirements, it is recommended that the following efforts be undertaken:

a) Investigate and evaluate alternative light sources more suited to the environmental requirements

b) Conduct further scientific research into the optimum CRDS approach to measuring mercury 


\section{Sensor Research \& Development Corporation DE-FC26-01FT41221}

concentrations

c) Address the engineering and packaging requirements including the development of an effective sampling interface

Finally, as evidenced by the results of this test, the development of this instrument (and other CEMs) would be enhanced by the opportunity to test the instrument under more controlled conditions prior to making the leap from laboratory to the pilot plant. That is, provide testing facilities with known and controllable mercury concentrations, exhaust gas composition, pressure, etc.

\section{Conclusions, Future Work, and Commercial Potential}

This report covers the work completed for Phase I, Phase II and the Pilot Field Test of the Cavity RingDown Spectroscopy Mercury Continuous Emission Monitor project.

This section of the report will present some preliminary commercialization potential, a conclusion and summary of the work performed under the first two phases of the project, the future worked planned, and the direction to bring the CRD instrument from the pilot scale field test to a marketable commercial product.

\section{Price Performance Matrix}

As an initial assessment of the market SRD has done a preliminary analysis of the market for commercially available mercury detectors. In an effort to make this market survey representative of available products SRD developed a list of mercury detectors that was quite varied. They range from larger bench top models to small hand-held models. They also range widely in the detection method used. The range of methods includes, gold amalgamation, atomic absorption, atomic fluorescence, and the cavity ring-down method employed by SRD. This should provide the most appropriate comparison of SRD's mercury detector to those available on the market.

SRD has developed a list of 10 Critical Performance Criteria, which includes 8 performance ratings and 2 cost ratings. Each performance criteria will have a scale where each detector is ranked from 1 to 5 , where 5 indicates superior performance and 1 indicates poor performance. For the cost portion, the rating system will again be 1 to 5 and here 5 represents high cost and 1 low cost. There will then be an overall performance score for each detector that will be the average score of the 8 performance criteria. There will also be an overall cost score, which will be the average of the 2 cost categories. The performance and cost criteria used for this market study are listed below: 


\section{Sensor Research \& Development Corporation DE-FC26-01FT41221}

\section{Critical Performance Criteria}

1) Speed of detection

2) Detection limits

3) Detection range

4) Capability to handle interferents

5) Portability and ease of use

6) Determination of absolute concentration

7) Accuracy of the measurement

8) Types of samples analyzed

9) Operating cost

10) Price

A brief description of each criteria and an example of the range used for the performance criteria is given below.

Speed of detection: This category rates each detector on the time taken from the sample input until the mercury concentration is actually reported. The scale will range from a real-time (seconds) readout to over 5 minutes for the reported concentration. For this category the actual scale that was used is; 1-5+ minutes, $2-2-5$ minutes, $3-1-2$ minutes, $4-<1$ minute, and $5-$ real time readout.

Detection limits: Each detector will be rated on the minimum amount of mercury that it can detect.

Detection range: This will rate the dynamic range, in terms of order of magnitudes that each detector can work over.

Capability to handle interferents: This category will rate each detector on its ability to handle any environmental sample without pre-processing or purification.

Portability and ease of use: The detectors will be rated on how portable they are, with the range being from very light hand-held devices to large laboratory bench top devices and also the ease of use for novice operators.

Determination of absolute concentration: This will rate each detector on the method used to determine concentration and the calibration procedure required for each device. The range will be from a self-calibration method to one requiring the device be sent back to the manufacturer for recalibration.

Accuracy of measurement: Each detector will be evaluated on how accurately they are able to determine the concentration of mercury.

Types of samples analyzed: This category will rate each detector on the types of samples that it can handle. For instance, can the device only handle gaseous samples or liquid samples, or can it handle gaseous, liquid, and solid samples. They will also be rated on whether the sample needs to be processed before introduction to the detector.

Operating cost: Each detector will be rated on the overall operational costs of the device. This will include materials needed to operate the device such as reagents, inert gas, etc. This category 


\section{Sensor Research \& Development Corporation DE-FC26-01FT41221}

will also take into account routine maintenance and part replacement recommended by the manufacturer.

Price: This will rate each detector on the overall retail price of the device.

The actual detectors chosen for this commercialization/market study were chosen from a wide variety and in such a way as to be representative of the various detectors available on the market today. SRD has also only included detectors that specialize in mercury rather than multiple substances.

The vendors and their various detectors that were used for this study are listed below:
Arizona Instruments:
Jerome 431-X Mercury Vapor Analyzer
Genesis Laboratory Systems, Inc.: ～GLSI Hg253 Portable Mercury Vapor Analyzer

Global Medical Instrumentation, Inc.: LA 254 LabAnalyzer Mercury Analyzer

Nippon Instruments Corporation:

1) Continuous Mercury Gas Monitor AM-2

2) Mercury/WA-4

\section{OhioLumex Company:}

P S Analytical:

Tekran Inc.:

\section{RA-915+ Mercury Analyzer}

1) PSA 10.025 Millennium Merlin System

2) Sir Galahad II Mercury Analyzer

1) Model 2500 CVAFS Mercury Detector

2) Model 2537A Mercury Vapor Analyzer

The above 7 vendors and 10 instruments will be compared with SRD's Cavity Ring-Down CEM instrument. For the purposes of this market study SRD will include two instruments for comparison. The only difference between the two will be the laser source that produces the $253.7 \mathrm{~nm}$ light used for mercury detection. SRD has a rather expensive, relatively non-portable, laser system because with present technology this is the only method to produce the, narrow band, monochromatic laser light, from a solidstate laser system, needed for the CRD instrument. The laser industry as a whole is presently trying to produce cheaper sources of UV laser light. This is due to the wide range of applications that would benefit from inexpensive sources of monochromatic UV radiation. In fact, improvement in laser technology has seen rapid development since the beginning of SRD's contract with DOE/NETL. A recent issue of Photonics (Photonics Spectra 36(2), pp. 24, 2002) has an article about a microfacet semiconductor laser operating at $401 \mathrm{~nm}$, with quite high power levels. While not fully into the UV yet, a researcher at NTT Basic Research Laboratories predicts that this new mirror fabrication technique could produce lasers that "may find a place as micron-sized blue and UV light sources." Even more recent developments have seen solid-state semi-conducting diode lasers operating below $380 \mathrm{~nm}$. Indeed, Coherent Incorporated has recently (November, 2003) released an $8 \mathrm{~mW}$, continuous wave, diode laser operating at $375 \mathrm{~nm}$. This laser has dimensions of only 1.75 inches by 8.6 inches. Additionally, Light Age Incorporated has completed a project sponsored in part by the NIST Advanced Technology Program, for the development of a less expensive, more compact, more efficient, and more reliable UV laser source than is presently on the market. Through this program they have produced a new solid-state, continuous wave, UV laser source that has the potential to be adapted to the requirements of the prototype CRD instrument being produced by SRD. As a result of this rapid development SRD will also include in the commercialization/market study an instrument with a reasonably priced solid-state laser as the source for 
the $253.7 \mathrm{~nm}$ light. The SRD instrument with the present laser source will be designated Model 1 and the instrument with a semi-conductor laser source will be Model 2. Table 3, shown below, tabulates the rating for each of the instruments in each category mentioned above as well as the overall score.

The numerical values from the table above have been used to create a graphical representation of the data. The plot, shown in Fig. 64, is a plot of overall price versus overall performance. In this plot performance increases from poor performance at 1 to superior performance at 5. The cost scale increases from inexpensive for 1 to expensive for 5. Lines have been drawn to separate the graph into quadrants. The quadrants have been labeled from 1 to 4 . Quadrant 1 represents the area of low cost and poor performance, quadrant 2 is the area of high cost and poor performance, quadrant 3 is the area of high cost and superior performance, and quadrant 4 represents the area of low cost and superior performance.

From the plot it can be seen that both of SRD's CRD models fall into the superior performance category. While Model 1 falls into quadrant 3, Model 2 falls into quadrant 4 where the instrument cost is low while exhibiting the highest rating of all the instruments in terms of performance. Even SRD's Model 1 exhibits far superior performance compared to almost every other instrument with the only drawback being the overall cost of the instrument. 


\begin{tabular}{|c|c|c|c|c|c|c|c|c|c|c|c|c|}
\hline \multirow{2}{*}{ 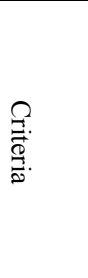 } & \multirow{2}{*}{ 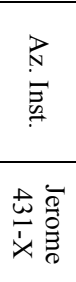 } & \multirow{2}{*}{ 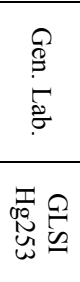 } & \multirow{2}{*}{ 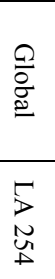 } & \multicolumn{2}{|c|}{ 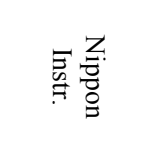 } & \multirow{2}{*}{ 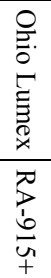 } & \multicolumn{2}{|c|}{ 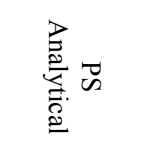 } & \multicolumn{2}{|c|}{ 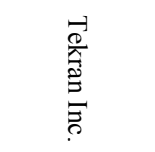 } & \multicolumn{2}{|c|}{$\begin{array}{l}\mathscr{\Omega} \\
\varrho \\
\Omega \\
0 \\
0\end{array}$} \\
\hline & & & & 永 & $\underset{⿱ 亠 乂}{\stackrel{1}{+}}$ & & $\frac{3}{9}$ & 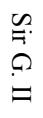 & $\begin{array}{l}\tilde{y} \\
\ddot{\delta}\end{array}$ & 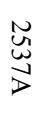 & $\begin{array}{l}3 \\
0 \\
0 \\
0 \\
=\end{array}$ & $\begin{array}{l}3 \\
\frac{3}{0} \\
\frac{0}{0} \\
\text { N }\end{array}$ \\
\hline 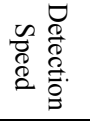 & 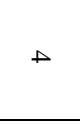 & ur & $\omega$ & $N$ & - & ur & $N$ & $N$ & N & ur & ur & ur \\
\hline 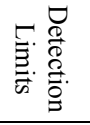 & - & - & $N$ & $u$ & $\omega$ & $u$ & $u$ & ur & 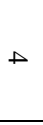 & ur & $\omega$ & $\omega$ \\
\hline 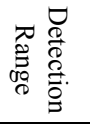 & $N$ & $\omega$ & $N$ & 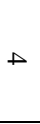 & 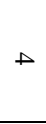 & $\omega$ & $\Delta$ & 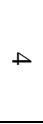 & $\omega$ & $\triangle$ & $\omega$ & $\omega$ \\
\hline 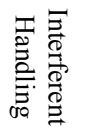 & $N$ & $\omega$ & $\omega$ & $N$ & $N$ & $u$ & - & - & - & - & ur & ur \\
\hline 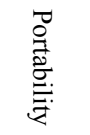 & ur & ur & - & $\omega$ & $\omega$ & 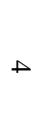 & - & $\omega$ & $\omega$ & $\omega$ & - & $\Delta$ \\
\hline 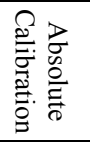 & - & - & 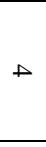 & 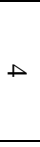 & $\omega$ & $\perp$ & 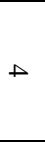 & 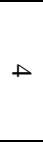 & $\omega$ & $\omega$ & ur & ur \\
\hline 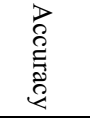 & $\omega$ & - & - & - & - & - & $N$ & $N$ & $\Delta$ & $\Delta$ & un & ur \\
\hline 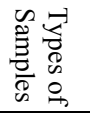 & - & - & ur & - & - & $\rightarrow$ & ur & - & ur & - & $\triangle$ & 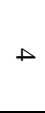 \\
\hline 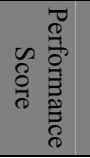 & $\underset{\infty}{\sim}$ & 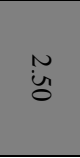 & $\stackrel{N}{\omega}$ & $\stackrel{N}{u}$ & $\stackrel{N}{N}_{n}^{N}$ & $\begin{array}{l}\omega \\
\infty \\
\infty\end{array}$ & $\dot{\omega}$ & $\stackrel{N}{u}$ & $\ddot{\omega}$ & $\underset{\tilde{u}}{w}$ & $\begin{array}{l}\omega \\
\infty \\
\infty\end{array}$ & $\overrightarrow{\dot{u}}$ \\
\hline 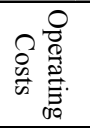 & $N$ & $\rightarrow$ & $N$ & $\omega$ & $\omega$ & $N$ & $\Delta$ & $\omega$ & $\omega$ & $\Delta$ & ur & $N$ \\
\hline 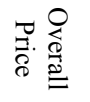 & - & $N$ & $\omega$ & $N$ & $\perp$ & $\omega$ & $\omega$ & $N$ & $N$ & $\omega$ & ur & $N$ \\
\hline 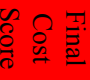 & $\overrightarrow{u_{r}}$ & $\omega$ & $\tilde{u}$ & u & ir & $\stackrel{n}{u}$ & $\tilde{w}_{r}$ & $\tilde{n}$ & $\tilde{\sim}$ & $\begin{array}{c}\omega \\
\dot{u}\end{array}$ & $\ddot{0}$ & $\stackrel{N}{0}$ \\
\hline
\end{tabular}

Table 3: Score of each instrument for all the different criteria and the final overall score for both performance and cost. 


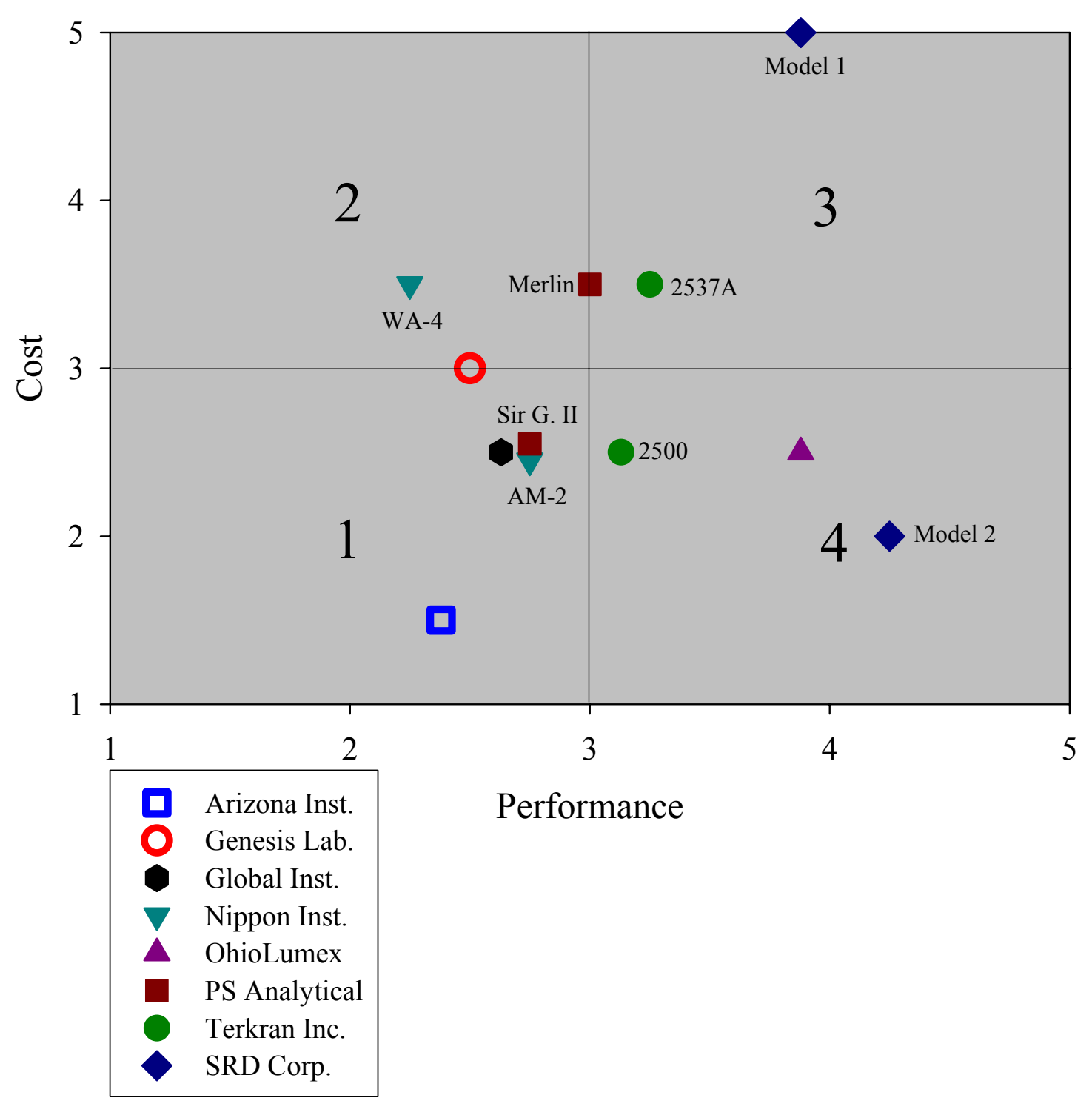

Figure 64: A plot of price versus performance using the data from Table 3. Also indicated on the plot are 4 quadrants. These quadrants mark 4 general areas, where quadrant 1 represents the low cost and poor performance area, quadrant 2 is high cost and poor performance, quadrant 3 is high cost and superior performance, and quadrant 4 represents the low cost and superior performance area. 


\section{Summary}

The development of a Continuous Emissions Monitor (CEM) for Mercury by the Sensor Research \& Development Corporation (SRD) has been a very successful project with the promise of a new type of CEM for mercury detection based on Cavity Ring-Down (CRD) spectroscopy. The project achieved all of the goals originally outlined in the specific milestones for Phases I \& II. The Pilot Field Test was successful in the sense that it confirmed the underlying technology; however, problems with the environmental conditions and complete access to the site precluded full completion of the field test. SRD is continuing to move the development of the CRD instrument forward toward commercialization.

The project began with the initial setup of the Alexandrite laser system and the ring-down cavity that were the essential components of the CRD instrument. The Alexandrite laser system was used to produce the $254 \mathrm{~nm}$ Ultra-Violet (UV) light needed for mercury detection. Upon observation of a ring-down event with the UV light an oxygen band, spectrally adjacent to the mercury, was detected and used as a diagnostic and for adjusting the CRD instrument. The oxygen transition was also used to maximize the operation of the CRD instrument and control software developed in-house by SRD software engineers. The actual detection of mercury in air was the next step in the effort where SRD scientists were able to observe the predicted naturally occurring isotopic structure, characteristic of the mercury transition. The limits of detection of mercury in air is into the low parts-per-trillion by volume ( $<10 \mathrm{pptr})$ range and the time for detection is generally in the low seconds time scale (approximately 10 seconds) or essentially real-time. This time for detection can be brought down significantly if needed. The present laser source has a repetition rate of $50 \mathrm{~Hz}$ and a single laser shot can be used to determine the mercury concentration, albeit at a significantly reduced precision, which would lead to a time interval between concentration data points of 20 milli-seconds.

The next phase of the project dealt with a variety of interferent species. The project is concerned with the detection of trace levels of mercury in the flue gas emissions from coal-fired power plants. However, the complex matrix of gases contained within the flue gas presents many problems to detectors on the market today. Flue gas contains a large number of combustion byproducts, all at concentration levels greater than mercury, presenting a very challenging environment in which to detect mercury. In dealing with this complex mixture of species most of the mercury detectors available today require extensive sample pretreatment before being able to detect the low levels of mercury. In particular, sulfur dioxide presents one of the biggest problems as it also absorbs UV radiation over the same spectral region as the mercury transition. SRD scientists were able to demonstrate accurate determination of mercury concentrations down into the pptr range even in the presence of sulfur dioxide concentrations that far exceeded those found in untreated flue gas emissions. It was also determined that none of the other components of flue gas effected the ability of the CRD instrument to accurately determine the mercury concentration. This was confirmed by individual testing of the components of flue gas as well as testing of a simulated flue gas stream.

One area of interest in mercury detection, throughout the power generation industry and environmental monitoring agencies, is that of speciation. This is the ability to not only determine the total gaseous mercury concentration in the flue gas stream but also determine the amounts of the different types of gaseous mercury. These are the elemental form $\left(\mathrm{Hg}^{(0)}\right)$ and the oxidized form $\left(\mathrm{Hg}^{(+2)}\right)$, both of which can occur in flue gas streams. This information is important in that mercury removal techniques for each species are quite different, as is the local/global environmental effects. SRD scientists have tested and are presently using a pyrolysis system to convert all the gas-phase oxidized mercury compounds to the elemental form. While the difference can be taken between the total and a non-pyrolyzed gas stream this 


\section{Sensor Research \& Development Corporation DE-FC26-01FT41221}

is not the ideal situation. SRD scientist would like to spectrally speciate between the two forms of mercury. This would give definitive results as to the concentration of each species. It was hoped that some spectral separation of the two forms would occur very close to the elemental mercury transition. However, thus far no such separation has been detected. SRD scientists and engineers are continuing work in this area to produce an even more desirable product for the power industry as well as other interested groups.

In addition to the experimental work accomplished, SRD scientists investigated theoretically modeling the mercury transition. This modeling is used to provide accurate mercury concentration levels within the CRD cavity at any temperature and pressure. Different temperatures and pressures create different spectral linewidths of the various mercury isotopomers. Differing linewidths result in different effective absorption cross-sections, which results in different mercury concentrations for the same total losses within the cavity. Therefore this modeling is essential to the operation of the CRD instrument under a variety of different conditions. The flue gas from coal-fired power plants can vary in temperature depending on the sampling point. The temperature in the combustor will reach about $1500^{\circ} \mathrm{C}$ while closer to the exhaust it will have dropped to around $150^{\circ} \mathrm{C}$ or less. In addition the mercury isotopic structure is much more pronounced and easier to identify at pressures below 1 atmosphere (760 torr), in the 100-400 torr range. The cavity in the CRD instrument can be held at pressures ranging from about 50 torr to above atmospheric pressure with a continuous sample flow rate of up to $1000 \mathrm{ccm}$.

SRD engineers have designed and built a gas sampling system that takes a sample gas stream from the flue gas stack, through a pyrolysis oven, into the CRD cavity where the mercury concentration is determined, and then returned to the flue gas stack in a continuous flowing system. All of the tubing, valves, fittings, and the CRD cavity itself are heat traced to maintain the sample gas stream at the temperature present in the flue gas stack. This ensures that there is no condensation and that the component ratios remain the same throughout the sampling system.

SRD has undertaken a number of long-term studies with the CRD instrument to determine its behavior over extended periods of time. A couple of problems were encountered during these long-term tests. The diode laser that seeds the Alexandrite laser was found to drift in frequency over time. This meant that the laser was no longer tuned to the peak of a mercury transition and hence was reporting inaccurate mercury concentrations. In addition to the drifting it was found that the diode seed laser would also occasionally mode hop. This is a condition where the output frequency of the diode seed laser would "hop" from one frequency to another that could be relatively far away in wavelength (on the order of $\mathrm{GHz}$ ). This again would result in inaccurate reporting of the mercury concentration.

Software engineers at SRD were able to correct the above-mentioned problems by adding additional subroutines to the LabVIEW program that controls the CRD instrument. These software changes have been implemented to not only correct the problems encountered with the diode seed laser, but also allow autonomous operation of the CRD instrument for extended periods of time while continuously reporting accurate mercury concentrations.

\section{Future Development}

The future of the CRD instrument's progress is presently very well defined. SRD is moving into a pilotscale testing phase has identified additional areas for further research, particularly in the future development of the instrument. An additional field test should be completed under more controlled conditions. Successful completion of the second pilot-scale test should lead to independent testing of the CRD instrument through the Environmental Protection Agency's (EPA) ETV program. The EPA through 
its Office of Research and Development (EPA-ORD) has instituted the Environmental Technology Verification (ETV) Program to verify the performance of innovative and improved technical solutions to problems that threaten human health or the environment. EPA created the ETV Program to substantially accelerate the entrance of new and improved environmental technologies into the domestic and international marketplaces. It is a voluntary, non-regulatory program. Its goal is to verify the environmental performance characteristics of commercial-ready technologies through the evaluation of objective and quality-assured data so that potential purchasers and permitters are provided with an independent and credible assessment of what they are buying and permitting. Testing through the ETV program would provide independent verification of the operation and performance of SRD's CRD instrument compared to other instruments presently or soon to be on the market.

SRD is continuing to work with Light Age Incorporated on the development of laser sources to produce the needed $253.7 \mathrm{~nm}$ UV radiation in a highly reliable, more compact system, at a significantly reduced cost compared to the laser system presently employed in the CRD instrument. All of which will result in a commercially viable product for the trace level detection of mercury. 


\section{Sensor Research \& Development Corporation DE-FC26-01FT41221}

\section{$\underline{\text { References }}$}

1. A. O'Keefe and D. A. G. Deacon, "Cavity ring-down optical spectrometer for absorption measurements using pulsed laser sources" Rev. Sci. Intstrum. 59 (12), 2544-2551 (1988).

2. G. Berden, R. Peeters, and G. Meijer, "Cavity ring-down spectroscopy: Experimental schemes and applications" Int. Rev. Phys. Chem. 19 (4), 565-607 (2000).

3. S. Spuler, M. Linne, A. Sappey, and S. Snyder, "Development of a cavity ring-down laser absorption spectrometer for detection of trace levels of mercury" Appl. Opt. 39 (15), 2480-2486 (2000).

4. G. Meijer, M. G. H. Boogaarts, R. T. Jongma, D. H. Parker, and A. M. Wodtke, "Coherent cavity ring-down spectroscopy" Chem. Phys. Lett. 217 (1,2), 112-116 (1994).

5. A. Jenouvrier, et al., "Fourier transform spectroscopy of the $\mathrm{O}_{2}$ Herzberg bands" J. Mol. Spec. 198, 136-162 (1999).

6. D. R. Linde, Handbook of Chemistry and Physics, $80^{\text {th }}$ ed. (CRC Press, Boca Raton, Fla., 1999).

7. W. G. Schweitzer, Jr., "Hyperfine structure and isotope shifts in the 2537 - $\AA$ line of mercury by a new interferometric method" J. Opt. Soc. Amer. 53 (9), 1055-1072 (1963).

8. E. J. Granite, Paper 12-1, Pittsburgh Coal Conference, Sept. 2002.

9. A. C. Vandaele, P. C. Simon, J. M. Guilmot, M. Carleer, and R. Colin, " $\mathrm{SO}_{2}$ absorption crosssection measurement in the UV using a Fourier transform spectrometer" J. Geophys. Res. 99 (D12), 25599-25605 (1994).

10. C. Y. R. Wu, B. W. Yang, F. Z. Chen, D. L. Judge, J. Caldwell, and L. M. Trafton, "Measurements of high-, room- and low-temperature photoabsorption cross-sections of $\mathrm{SO}_{2}$ in the 2080- to 2950-Å region, with application to Io" Icarus 145, 289-296 (2000).

11. S. L. Manatt and A. L. Lane, "A compilation of the absorption cross-sections of $\mathrm{SO}_{2}$ from 106 to 403 nm” J. Quant. Spectrosc. Radiat. Transfer 50 (3), 267-276 (1993).

12. J. Rufus, G. Stark, P. L. Smith, J. C. Pickering, and A. P. Thorne, "High-resolution photoabsorption cross-section measurements of $\mathrm{SO}_{2}, 2: 220$ to $325 \mathrm{~nm}$ at $295 \mathrm{~K}$ " J. Geophys. Res. 108 (E2), 5-1 - 5-5 (2003).

13. D. P. Baldwin, S. J. Bajic, D. E. Eckels, and D. S. Zamzow, "Testing of continuous sampling Air-ICP and mercury systems as continuous emissions monitors at the Diagnostic Instrumentation and Analysis Laboratory" Ames Laboratory-USDOE, Report IS-5144, (2001).

14. L. S. Rothnam, and J. Schroeder, "The HITRAN (2000) Molecular Spectroscopic Database and JHAWKS (2001)", Cambridge, MA. 


\section{Sensor Research \& Development Corporation DE-FC26-01FT41221}

15. G.C. King and A. Adams, "An accurate determination of the lifetime of the $6^{3} \mathrm{P}$ state in mercury using a new electron-photon delayed coincidence apparatus," J. Phys. B: Atom. molec. Phys. 7, 1712-1718 (1974).

16. J. P. Jacobs and R. B. Warrington, "Measurement of pressure broadening and shift for Hg 254 nm line by $\mathrm{N}_{2}$," Bul. Am. Phys. Soc. 44, Part I, $729-730$ (1999). Presented at the American Physical Society Centennial Meeting, Atlanta, GA.

17. E.E. Whiting, "An empirical approximation to the Voigt profile," J. Quant. Spectrosc. Radiat. Transfer 8, 1379-1384 (1968).

18. VICI Metronics Inc., Technical Note 1001.

19. Brooks Rand Model III Atomic Fluorescence Spectrometer operations manual.

20. Tekran Inc. publication: "Tips on Making Saturated Mercury Vapor Injections."

21. Fabio J. Mazzotti, Chuji Wang, Christopher B. Winstead, and George P. Miller. "Analytical Atomic Cavity Ringdown Spectroscopy - A Comparison of Three different Atomization Sources for the Detection of Mercury." In preparation.

22. Fabio J. Mazzotti, "Investigation of Inductively Coupled Plasma as an Atomization Source for Analytical and fundamental Measurements using Cavity Ringdown Spectroscopy" PhD Thesis Mississippi State University (2004).

23. C. Wang, F. J. Mazzotti, S. P. Koirala, C. B. Winstead and G. P. Miller, "Measurement of OH Radicals in a Low-Power Inductively Coupled Plasma by Cavity Ringdown Spectroscopy," Applied Spectroscopy, 58, 734 (2004).

24. Chuji Wang, Fabio J. Mazzotti, George P. Miller and Christopher B. Winstead. "Isotopic Measurement of Uranium Using Inductively Coupled Plasma Cavity Ringdown Spectroscopy" Applied Spectroscopy, 57, 1167 (2003).

25. D. R. Wagner, C. B. Winstead, and G. P. Miller "Infrared Cavity Ringdown Spectroscopy", in Handbook of Vibrational Spectroscopy, J.M. Chalmers and P.R. Griffiths (Eds), John Wiley \& Sons, Ltd, Volume 1, pp. 866-880 (2002).

26. C. Wang, F. J. Mazzotti, C. B. Winstead, and G. P. Miller. "ICP-CRDS - Cavity Ringdown Spectroscopy as a plasma diagnostic." Applied Spectroscopy. 50, 386 (2002).

27. S. Tao, F. J. Mazzotti, C. B. Winstead, and G. P. Miller, "Determination of elemental mercury by cavity ringdown spectrometry" Analyst 125, 1021 (2000).

28. G. P. Miller and C. B. Winstead, Cavity Ringdown Laser Absorption Spectroscopy, pp. 1073410750, Encyclopedia of Analytical Chemistry: Instrumentation and Applications. Ed-in-Chief Dr. Robert A. Meyers. John Wiley \& Sons Ltd. (2000).

29. C. B. Winstead, F. J. Mazzotti, J. Mierzwa, and G. P. Miller, Preliminary results for Electrothermal Atomization-Cavity Ringdown Spectroscopy (ETA-CRDS). Anal. Commun. 36277 (1999). 
30. G. P. Miller and C. B. Winstead. ICP-Cavity Ringdown Spectroscopy. J. Anal. Atomic Spectra 9, 907 (1997).

31. C. C. Carter, A Cavity Ringdown Spectroscopy Mercury Continuous Emission Monitor, Draft Final Technical Report DOE DE-FC26-01FT41221 (2004).

32. D. P. Baldwin, D. S. Zamzow, D. E. Eckels, and G. P. Miller, A Continuous Sampling air-ICP for Metals Emission Monitoring. Proc. Of SPIE 3853 (1999).

33. D. P. Baldwin, D. S. Zamzow and G. P. Miller, AOTF-Echelle spectrometer for air-ICPAES continuous emission monitoring of heavy metals \& actinides. Proc Of SPIE 3534,59(1998). 